\title{
Surface reflectance analysis of small bodies on different scales
}

\author{
Dissertation \\ zur Erlangung des mathematisch-naturwissenschaftlichen Doktorgrades \\ "Doctor rerum naturalium" \\ der Georg-August-Universität Göttingen \\ im Promotionsprogramm PROPHYS \\ der Georg-August University School of Science (GAUSS)
}

vorgelegt von

Nafiseh Masoumzadeh Jouzdani

aus Esfahan, Iran

Göttingen, 2015 
Betreuungsausschuss

Prof. Dr. Stefan Dreizler

Institut für Astrophysik, Georg-August-Universität Göttingen, Germany

Prof. Dr. Ulrich Christensen

Max-Planck-Institut für Sonnensystemforschung, Göttingen, Germany

Dr. Hermann Böhnhardt

Max-Planck-Institut für Sonnensystemforschung, Göttingen, Germany

Mitglieder der Prüfungskommision

Referent: Prof. Dr. Stefan Dreizler

Institut für Astrophysik, Georg-August-Universität Göttingen, Germany

Korreferent: Prof. Dr. Ulrich Christensen

Max-Planck-Institut für Sonnensystemforschung, Göttingen, Germany

Weitere Mitglieder der Prüfungskommission:

Prof. Dr. Manfred Schüssler

Max-Planck-Institut für Sonnensystemforschung, Göttingen, Germany

Prof. Dr. Wolfram Kollatschny

Institut für Astrophysik, Georg-August-Universität Göttingen, Germany

Prof. Dr. Ansgar Reiners

Institut für Astrophysik, Georg-August-Universität Göttingen, Germany

Prof. Dr. Andreas Pack

Geowissenschaftliches Zentrum, Abteilung Isotopengeologie, Georg-August-Universität Göttingen, Germany

Tag der mündlichen Prüfung: 09.04.2015 


\section{Bibliografische Information der Deutschen Nationalbibliothek}

Die Deutsche Nationalbibliothek verzeichnet diese Publikation in der Deutschen Nationalbibliografie; detaillierte bibliografische Daten sind im Internet über http: //dnb . d-nb . de abrufbar.

ISBN 978-3-944072-12-8

uni-edition $\mathrm{GmbH} 2015$

http://www.uni-edition.de

(C) Nafiseh Masoumzadeh Jouzdani

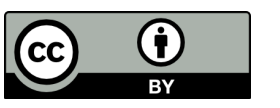

This work is distributed under a

Creative Commons Attribution 3.0 License

Printed in Germany 



\section{Contents}

$\begin{array}{lr}\text { Summary } & 9\end{array}$

$\begin{array}{lll}1 & \text { Introduction } & \mathbf{1 1}\end{array}$

1.1 The small bodies in the solar system . . . . . . . . . . . . . . . . 11

1.2 Space missions to asteroids and comets . . . . . . . . . . . . . . . . . 16

$1.3 \quad$ Space weathering of asteroid surfaces . . . . . . . . . . . . . . . . 19

1.4 The reflectance analysis of small bodies . . . . . . . . . . . . . . 20

1.5 Motivation of the thesis . . . . . . . . . . . . . . . . . 22

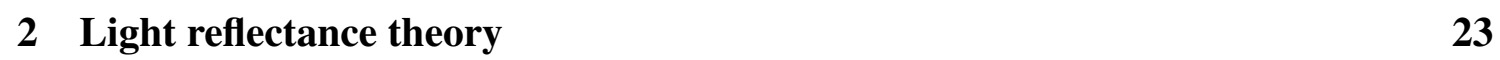

2.1 General terminology . . . . . . . . . . . . . . . . . . 23

2.1 .1 Scattering Surface . . . . . . . . . . . . . . . . 23

2.1 .2 Geometrical angles . . . . . . . . . . . . . . . . 23

2.1 .3 Reflectance . . . . . . . . . . . . . . . . . . . . . . . . . . . . . . . 24

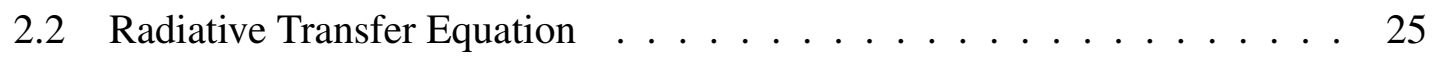

$2.2 .1 \quad$ Single-particle light scattering model . . . . . . . . . . . . 25

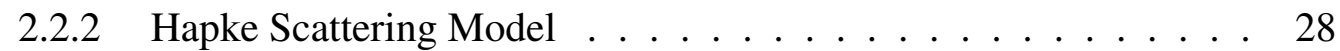

$2.2 .2 .1 \quad$ Single Scattering . . . . . . . . . . . . . . . . 29

$2.2 .2 .2 \quad$ Multiple scattering . . . . . . . . . . . . . . . . . . . . . . 30

2.2 .2 .3 Opposition Effect $\ldots \ldots \ldots \ldots . \ldots . \ldots . \ldots 31$

2.2.2.4 Macroscopic roughness . . . . . . . . . . . . . . . 34

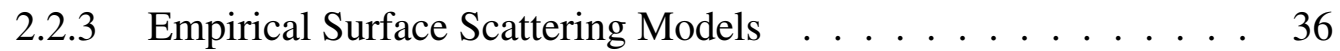

$2.2 .3 .1 \quad$ Lambert Model . . . . . . . . . . . . . . . . . . . . . . . . . . . . . . 36

$2.2 .3 .2 \quad$ Minnaert Model . . . . . . . . . . . . . . . . . . 36

2.2.3.3 Lommel-Seeliger Model . . . . . . . . . . . . . . . . . 37

2.2.3.4 Lunar-Lambert Model . . . . . . . . . . . . . . . . . 37

2.3 Quantities related to disk-integrated reflectance . . . . . . . . . . . . . . 37

2.3 .1 The geometric albedo . . . . . . . . . . . . . . . . 37

2.3 .2 The phase integral $\ldots \ldots \ldots \ldots$. . . . . . . . . . . 38

2.3 .3 The Bond albedo . . . . . . . . . . . . . . . . . . . . . 38

2.3 .4 The Hapke disk-integrated model . . . . . . . . . . . . . 38

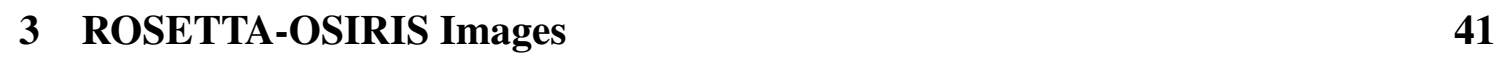

3.1 ROSETTA, the ESA's mission . . . . . . . . . . . . . . . . . 41

3.2 OSIRIS, the camera system $\ldots \ldots \ldots \ldots \ldots$. . . . . . . . . . . . . . . . . . . . . . 45

3.3 Rosetta asteroid fly-bys $\ldots \ldots \ldots$. . . . . . . . . . . . . . 48 
$3.3 .1 \quad$ Rosetta fly-by at Asteroid (2867) Steins . . . . . . . . . . . . . . 48

3.3.2 Rosetta fly-by at Asteroid (21) Lutetia . . . . . . . . . . . . . . . 48

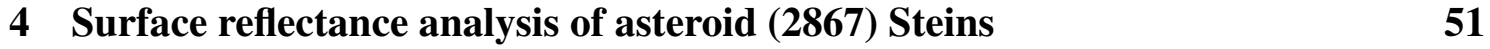

4.1 Overview of Steins' surface . . . . . . . . . . . . . . . . . . 51

4.2 Data extraction . . . . . . . . . . . . . . . 53

4.3 Fitting algorithm . . . . . . . . . . . . . . . . 55

4.4 Disk-integrated Reflectance . . . . . . . . . . . . . . . . . . . . . 55

4.4 .1 Disk-integrated phase function . . . . . . . . . . . . . . . 55

$4.4 .2 \quad$ Hapke disk-integrated modeling . . . . . . . . . . . . . . . . . . 56

4.5 Disk-Resolved Reflectance evaluation . . . . . . . . . . . . . . . . 57

4.5 .1 Minnaert modeling . . . . . . . . . . . . . . . . 57

4.5.2 Hapke disk-resolved modeling . . . . . . . . . . . . . . . . . . 59

4.6 Error analysis of the modeled Hapke parameters . . . . . . . . . . . . . . 65

4.7 Comparison with previous analyses $\ldots \ldots \ldots 66$

$5 \quad$ Surface Reflectance Analysis of Asteroid (21) Lutetia 69

5.1 Overview of Lutetia's Surface . . . . . . . . . . . . . . . . . . 69

5.2 Data extraction . . . . . . . . . . . . . . . . . . . 74

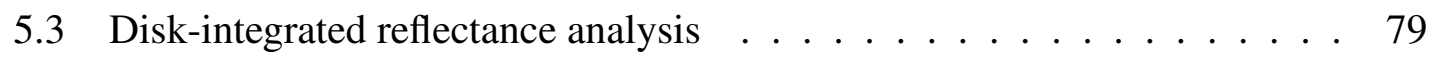

5.3 .1 Disk-integrated phase function . . . . . . . . . . . . . . . . 79

5.3 .2 Disk-integrated color . . . . . . . . . . . . . . . . . 80

5.3 .3 Hapke disk-integrated modeling . . . . . . . . . . . . . . . . . 81

5.4 Disk-Resolved reflectance analysis . . . . . . . . . . . . . . . 86

5.4 .1 Minnaert model . . . . . . . . . . . . . . . . . 86

$5.4 .2 \quad$ Hapke disk-resolved modeling . . . . . . . . . . . . . . . 99 91

5.5 Error analysis of the nominal Hapke parameters . . . . . . . . . . . . . . 94

5.6 Surface variation analysis $\ldots \ldots \ldots \ldots$. . . . . . . . . . . . 97

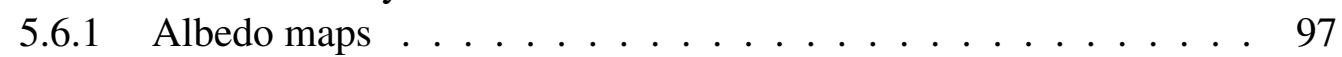

$5.6 .2 \quad$ Phase Ratio Maps . . . . . . . . . . . . . . . . . . . . . . . . . . . . . . . . . . . . . . . .

5.6.3 Color Ratio Maps . . . . . . . . . . . . . . . . . . . . . . 101

6 Comparison of Asteroids Steins and Lutetia with other small bodies 109

6.1 Overview of small bodies' surface reflectance . . . . . . . . . . . . . . . 109

6.2 Comparison between photometric properties of asteroids and cometary nuclei . . . . . . . . . . . . . . . . 114

6.3 Comparison with laboratory measurements . . . . . . . . . . . . 118

\begin{tabular}{lll}
\hline 7 & Conclusions & 125
\end{tabular}

\begin{tabular}{lr}
\hline Bibliography & 127
\end{tabular}

\begin{tabular}{|l|l|}
\hline A The 2d polynomial fitting to table data of the roughness parameter & 139
\end{tabular}

$\begin{array}{ll}\text { Scientific contributions } & 141\end{array}$

\begin{tabular}{ll}
\hline Acknowledgements & 143
\end{tabular} 
\begin{tabular}{ll}
\hline Curriculum vitae & 145
\end{tabular} 



\section{Summary}

Studying reflectance from the surface of asteroids and other atmosphereless small bodies provides information not only about the optical properties across the surface, but also it allows understanding the physical properties of the regolith on the surface. Resolved images, returned by spacecraft from asteroids and cometary nuclei, have amplified the interest to study the surface reflectance of small bodies in the solar system.

The aim of this thesis is to model the surface reflectance of small bodies using spacecraft data i.e. asteroids Steins and Lutetia. The overall and local variation of brightness from the surface covers with different local geometries are controlled by the optical, structural, and physical properties of the surface material. Two photometric models are frequently utilized to model the scattering of light from planetary and small bodies surfaces, the one of Hapke (Hapke, 1993) and the one of Minnaert (Minnaert, 1941). In both approaches, the intensity is modeled in terms of viewing and illuminating angles with a number of photometric parameters. The Minnaert model is an empirical photometric function with two parameters, the Minnaert albedo and the limb-darkening coefficient, representing the light scattering behavior across the surface. The Hapke photometric function, is a semi-empirical model and is mathematically more complicated which is composed of five coupled parameters, known as Hapke parameters. This work focuses on the application of the Minnaert and Hapke modeling to find the optimum values of their photometric parameters from the surface reflectance of two asteroids Steins and Lutetia.

I used OSIRIS (Optical, Spectroscopic, and Infrared Remote Imaging System) images acquired during Rosetta encounter on September of 2008 and July of 2010 of Steins and Lutetia, respectively.

I first develop a tool to extract reflectance from the spacecraft images and to link it with the relevant scattering geometry and then to constrain the photometric parameters from the observational data. For this purpose, I employ the shape models of the asteroids and associate them with spacecraft and planetary ephemerides as well as with spacecraft orientation. By co-aligning the shape model and spacecraft images, I obtain the information needed to model the light scattering parameters.

I validate first my tool and the approach by applying to the Steins images which were also analyzed through Hapke disk-resolved photometry (Spjuth et al., 2012). The comparison shows a good agreement between my Hapke solutions and those retrieved by Spjuth et al. (2012).

Then, I performed a full reflectance analysis for asteroid Lutetia, using disk-integrated and disk-resolved data of this object in different filters. This approach, which is applied on the OSIRIS images covering extensive phase angle and visible wavelength ranges, allows to constrain the regolith properties of Lutetia in the context of reflectance measurement as well as the reflectance variation across the surface. 
I construct the disk-average reflectance spectra of Lutetia at phase angle $7.7^{\circ} 269.3$ $\mathrm{nm}$ and $989.3 \mathrm{~nm}$, which show constant spectral shape. The small spectral slope $(2.9 \pm 0.2)$ $\%$ per $100 \mathrm{~nm}$ is consistent with that obtained from high-resolution spectra of Lutetia reported by VIRITIS (Coradini et al., 2011). I also analyze the variation in albedo, color, and phase function over the surface of Lutetia. The analysis reveals that Lutetia's surface is homogeneous in reflectivity. No heterogeneity in color is seen across the surface of Lutetia. The ratio of images for different viewing geometries also does not present any variation that can be attributed to phase function and/or roughness alternation.

I placed the modeled results of Steins and Lutetia in a wider perspective with all photometric parameters published for asteroids and cometary nuclei from spacecraft images. The modeled Hapke parameters indicate the reflectance is relatively high related to single scattering. The Minnaert modeling of Steins illustrates the disk of object as a limb-darkened surface. While, based on the Minnaert parameters modeled for Lutetia, the surface of Lutetia is not limb-darkened. The Hapke solutions for Lutetia shows moderate value for all parameters, apart from the high values of opposition surge parameters $\left(\mathrm{B}_{0}=1.79 \pm 0.08\right.$ and $\left.\mathrm{h}=0.050 \pm 0.003\right)$. It is noted that $\mathrm{I}$ found the disk-integrated phase function of Lutetia is similar to average S-type asteroids as well as the Lutetia's Hapke parameters. This similarity mostly originates from two Hapke parameters the single scattering albedo ( $\mathrm{SSA}=0.226 \pm 0.002)$ and the asymmetry factor $(\mathrm{g}=-0.28 \pm 0.01)$.

I also compare my Hapke results for Lutetia and Steins with the available laboratory reflectance measurements from soil and meteorite samples. The reflectance measurement of asteroids Lutetia and Steins can be represented by the chromium oxide samples in packed and loose states (Shepard and Helfenstein, 2007), respectively. I conclude that the regolith on the surface of Lutetia is more condense, less porous than the surface layer of Steins. The comparison also suggests that among the available meteorite samples, the modeled reflectance from the Allende meteorite sample (Beck et al., 2012) fits best to the measured reflectance from the surface of Lutetia. While such similarity should not be seen as a proof for the meteorite analogy of Lutetia, it can provide constraints on the grain size and albedo of Lutetia. In fact, the consistency between the reflectance data of the Allende meteorite and Lutetia might appear due to the similarity between the fine grain size distribution and moderate albedo of the sample and the regolith on the Lutetia. 


\section{Introduction}

\subsection{The small bodies in the solar system}

Beside the major members of the solar system, the eight planets and their satellites (or moons), numerous small bodies such as asteroids, comets, and Kuiper belt objects are orbiting the Sun. They are considered the remnants of the plantesimals formed in the early period of the solar system. Although the small bodies have encountered a variety of events, they contain information about the environment and the physical conditions of early solar system. The physical understanding of these objects forms a necessary base to unravel the origin of the solar system.

Asteroids are small airless objects, usually showing no outflow of gas and dust. The orbital location of most of the asteroids is between heliocentric distances of 2.1 and 3.3 AU, known as the main asteroid belt. In fact, the main belt asteroid in the inner solar system, between the orbits of Mars and Jupiter, is the largest source for asteroids. The asteroids are classified into a number of groups mostly related to surface composition, however the sub-classification is different in the various literature. In Table 1.1, the asteroids are categorized in taxonomic classes based on their reflectance spectra together and albedo (Pater and Lissauer, 2001).

The most abundant category of asteroids is the $\mathrm{C}$ type or carbonaceous asteroids (40\% of the known asteroids) which are dominant in the outer belt ( $>2.7 \mathrm{AU})$. The $\mathrm{C}$ type asteroids are dark objects with geometric albedo less than 0.1 and flat spectral shape to $0.4 \mu \mathrm{m}$. In some $\mathrm{C}$ type asteroids, there is evidence of an absorption band around $3 \mu \mathrm{m}$, serving as a sign of water. The similarity between spectra of carbonaceous chondrite meteorites (CI, CM) and the C-type asteroids suggests that this class of asteroids are composed of carbon-rich material. The carbonaceous chondrite meteorites are convinced to be representatives of primitive material which has been gently heated and condensed in low temperature environment.

The next largest group of asteroids (30-35\%) are the S-class or stony asteroids. These bodies have a moderate geometric albedo between 0.1 to 0.3 . The spectra of S-class asteroids show two weak or/and moderate absorption bands near 1 and $2 \mu \mathrm{m}$, indicative of pyroxene $\left((\mathrm{Fe}, \mathrm{Mg}) \mathrm{SiO}_{3}\right)$ and olivine $\left((\mathrm{Fe}, \mathrm{Mg})_{2} \mathrm{SiO}_{4}\right)$, respectively. A strong absorption feature around $0.7 \mu \mathrm{m}$ is also suggestive of iron oxides. With this diversity of mineralogies, it is puzzling to find an analogous meteorite. Several $\mathrm{S}$ type asteroids were found to have spectra similar to ordinary chondrites; however, the spectral red slope of S-class asteroids is not seen in ordinary chondrites.

In addition to these two major classes, the outer belt is occupied with the low-albedo $\mathrm{D}$ and $\mathrm{P}$ classes (5-10\%). The $\mathrm{D}$ and $\mathrm{P}$ type asteroids are extremely dark and may state 
Table 1.1: The classification of asteroid types, modified from table 9.4 in Pater and Lissauer (2001)

\begin{tabular}{llll}
\hline $\begin{array}{l}\text { Asteroid } \\
\text { type }\end{array}$ & $\begin{array}{l}\text { Spectral } \\
\text { features }\end{array}$ & $\begin{array}{l}\text { Meteorite } \\
\text { analogues }\end{array}$ & Location \\
\hline
\end{tabular}

\section{Low-albedo:}

\begin{tabular}{|c|c|c|}
\hline $\begin{array}{l}\text { C Carbonaceous } \\
\text { asteroids }\end{array}$ & $\begin{array}{l}\text { spectrum flat, UV absorp- } \\
\text { tion feature of } 0.4 \mu \mathrm{m}\end{array}$ & $\mathrm{CI}$ and $\mathrm{CM}$ meteorites \\
\hline $\mathrm{D}$ & $\begin{array}{l}\text { Red featureless spectrum } \\
\text { possibly correspond to or- } \\
\text { ganic material }\end{array}$ & \\
\hline $\mathrm{P}$ & $\begin{array}{l}\text { rather reddish spectrum; } \\
\text { flat spectral shape similar } \\
\text { to } \mathrm{M} \text { type asteroids }\end{array}$ & \\
\hline $\mathrm{K}$ & & $\mathrm{CV}$ and $\mathrm{CO}$ meteorites \\
\hline $\mathrm{T}$ & $\begin{array}{l}\text { Modest absorption feature } \\
\text { of } 0.85 \mu \mathrm{m} \text {; flat spectrum } \\
\text { at longer wavelengths }\end{array}$ & $\begin{array}{l}\text { Unknown composition, } \\
\text { likely highly altered C } \\
\text { types }\end{array}$ \\
\hline
\end{tabular}

Moderate-albedo:

\begin{tabular}{|c|c|c|}
\hline S Stony asteroids & $\begin{array}{l}\text { Absorption band at } 0.7 \\
\mu m \text {; Weak absorption fea- } \\
\text { tures near } 1 \text { and } 2 \mu m\end{array}$ & \\
\hline M & $\begin{array}{l}\text { featureless flat to reddish } \\
\text { spectrum }\end{array}$ & Stony-iron or iron \\
\hline Q & $\begin{array}{l}\text { absorption feature around } \\
0.7 \mu m\end{array}$ & $\begin{array}{l}\text { Ordinary }(\mathrm{H}, \mathrm{L}, \mathrm{LL}) \text { chon- } \\
\text { drite meteorites }\end{array}$ \\
\hline A & $\begin{array}{l}\text { strong absorption feature } \\
\text { near } 1 \mu m \text {; extremely red- } \\
\text { dish spectrum around } 0.7 \\
\mu m\end{array}$ & \\
\hline V & $\begin{array}{l}\text { strong absorption feature } \\
\text { around } 0.7 \mu m \text { and near } 1 \\
\mu m\end{array}$ & Basaltic achondrites \\
\hline $\mathrm{R}$ & $\begin{array}{l}\text { In-between spectrum of A } \\
\text { and V classes }\end{array}$ & Olivine-rich achondrites \\
\hline
\end{tabular}

Major population in inner to central belt

Central belt

Outer belt beyond $2.7 \mathrm{AU}$

Utmost outer belt and Troajns

Outer and extreme outer belt

Asteroid (4) Vesta and a few Earthcrosser

Main belt (rare)

\section{High-albedo:}

E

Featureless, flat to moder- Enstatite asteroids Near inner edge of ately reddish spectrum

belt 
even more primitive bodies than the carbonaceous $\mathrm{C}$ type asteroids. The spectra of both $\mathrm{D}$ and $\mathrm{P}$ types are featureless, but red which might be associated with organic compounds.

Another common asteroid type are M-class asteroids which have spectral features similar to iron-nickel metal. Their spectra are similar to iron meteorites and enstatite chondrites. The geometric albedo of $\mathrm{M}$ type asteroids falls into the range of moderate albedo $(\approx 0.1-0.2)$. A small number of very bright asteroids (albedo range 0.3-0.6) are classified as $\mathrm{E}$ type asteroids. They gather near the inner edge of the belt and show slightly reddish spectra mainly caused by enstatite or iron-poor silicates. The spectra of $\mathrm{M}$ and E type asteroids in the visible wavelength give us clues that these classes have melted during substantial thermal process.

There are a number of asteroids which cannot be categorized into the case of main group of asteroids described above. Hence, several small asteroid classes are defined, some of which have only a few asteroids as members such as R, Q, and V type groups. The R-class is formed with a single member, asteroid (349) Demnowska. Its reflectance spectra demonstrates the large amount of olivine without metals. The Q-class is one of very few group of asteroids whose spectra has an exact resemblance to ordinary chondrites. The V-class asteroids are excellent analogs for the the basaltic achondrite meteorites. Asteroid (4) Vesta is a member of the V type group that it is found to be a parent body of the HED (Howardite-Eucrite-Diogenite) meteorites. Along with Vesta, a small number of $\mathrm{V}$ type asteroid which have similar orbital elements as those of Vesta.

To explain the formation of the asteroid belt, I delivered the main points of the review by (Petit et al., 2002), which gives us the most significant aspects observed in the asteroid belt as follow;

- Strong dynamical excitation: Compared to the planets, the orbital elements of asteroids such as eccentricity and inclination are much larger. In the inner belt, the median eccentricity $e_{m}$ is 0.15 and the median inclination $i_{m}$ is close to $6^{\circ}$, in the central belt $e_{m}$ is slightly reduced to 0.14 , while $i_{m}$ increases to $10.7^{\circ}$. In the outer belt, $e_{m}$ is 0.1 , except for bodies in the resonances, i.e. lower than in other region of the belt due to the dynamical instability caused by Jupiter, while $i_{m}$ tends to increase a little to $12.1^{\circ}$. The region at low e $(<0.05)$ and $i\left(<2^{\circ}\right)$ is empty, despite orbits in this location could be dynamically stable over the age of solar system.

- Large mass depletion: The total mass of bodies in the asteroid belt is less than $10^{-3} M_{\oplus}$ at the present. The amount is not comparable with the mass needed to produce a planet by accretion of the protoplanetary disk. This indicates that mass deficiency most likely occurred because of some dynamical mechanisms like after the formation of asteroids. The mass deficiency is lower in the central belt compared with the inner and outer belt.

- Radial mixing of asteroid types: The distribution of of taxonomic classes of asteroids with respect to heliocentric distance (Figure 1.2) demonstrates that there is an obvious trend among asteroids types versus distance from the Sun. The high albedo E class asteroids are the major group in the innermost part of the asteroid belt (peaking at $2 \mathrm{AU}$ ). The next group, $S$ type asteroids dominate in the inner belt, $M$ types prevail in the central part of the belt, while $C$ type objects expand from center to near the outer edge of main belt (peaking at 3AU). D and P type asteroids 


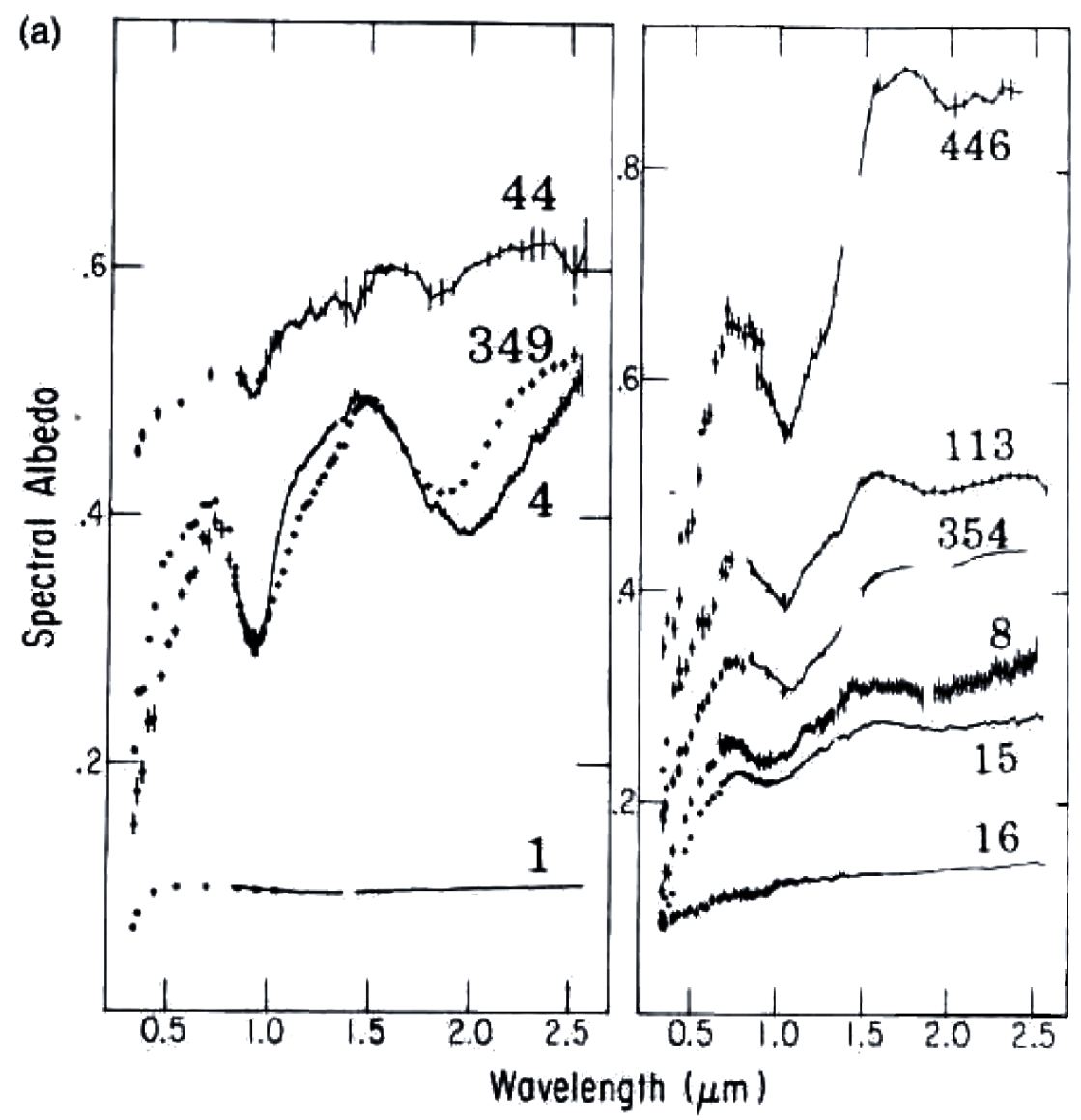

Figure 1.1: A number of asteroid reflectance spectra as indicatives of asteroid taxonomic classes: Left panel: 1 Ceres (C type), 4 Vesta (V type), 44 Nysa (E type), and 349 (Dembowska (R type). Right panel: 8 Flora (S type), 15 Eunomia (S type), 16 Pysche (M type), 113 Amalthea (S type:offset by +0.1 ), 354 Eleonora (S type: offset by +0.1 ), and 446 Aetarnitas (A type; offset by +0.2 ) adapted from figure 9.17 in Pater and Lissauer (2001).

are located only in the outer belt and among Trojan asteroids (peaking at 5.2 AU and 4 AU, respectively). The systematic arrangement of asteroid types with heliocentric distance suggests that there is a primordial distribution modified by a series of evolutionary or dynamical events.

It is widely accepted and suggested by several simulations (Wetherill and Stewart, 1993; Weidenschilling and Davis, 2001) that the mechanism of gravitational perturbation from embryos describes the formation of asteroid belt reasonably well. The fact that large embryos have survived from planetary formation is concluded from current modeling. The early asteroid belt contained sub-lunar- to martian-size embryos that interacted with each other gravitationally until they reached the proximity of Jupiter. In this phase, some embryos were ejected from the solar system, while a small portion of them remained in the stable part of the belt, but in eccentric and inclined orbits.

Comets are small icy bodies, in highly eccentric orbits, partially very elongated around the Sun and become active when approaching the Sun. The population of comets is di- 


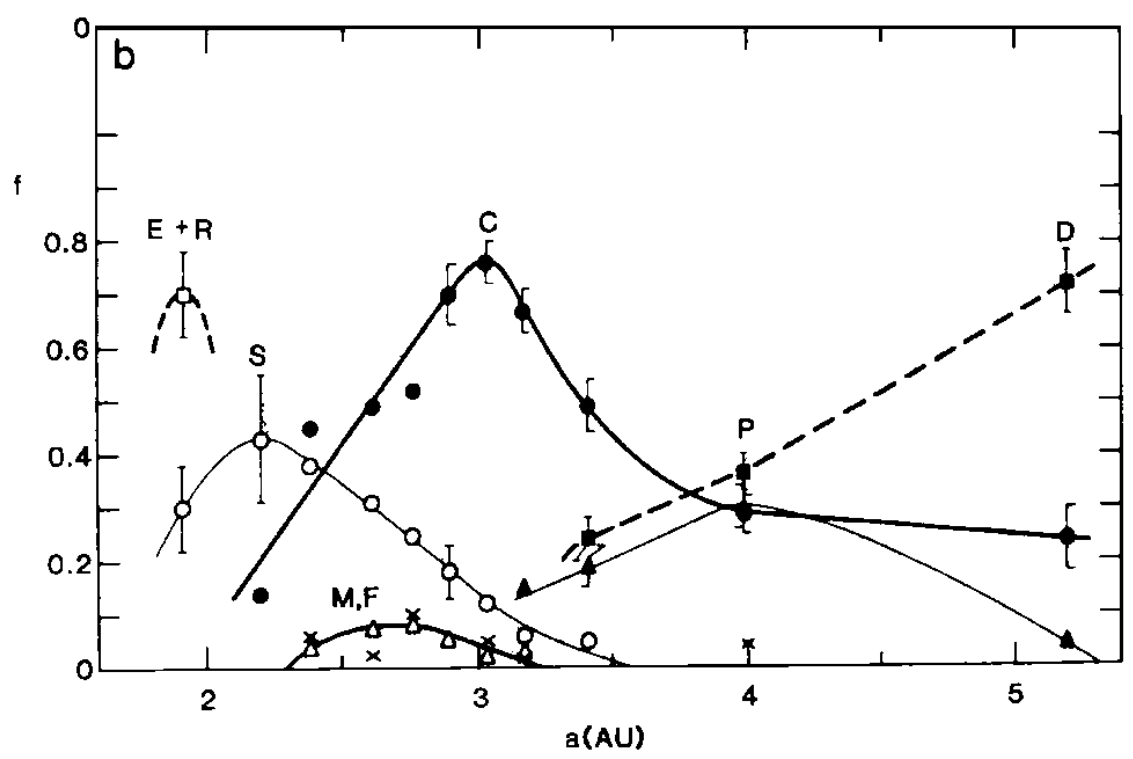

Figure 1.2: The relative distribution of asteroid types with respect to heliocentric distance. Types C, M, E, P, S, R, F, and D are illustrated with different symbols. Smooth curves are depicted better the nonrandom distribuation. The figure is taken from (Gradie and Tedesco, 1982)

vided into two categories as stated by their orbital elements. The division is based on the ratio of inclination versus semi-major axis of known cometary orbit. Hence, the two major groups of cometary orbits are ecliptic comets (low inclination distribution) and nearly-isotropic comets (broad inclination distribution). The most abundant population of the known ecliptic comets is the Jupiter-family comets, which can cross the orbit of Jupiter and are dynamically controlled by that planet. The nearly-isotropic comets are subdivided into two groups of dynamically new comets and returning comets. According to the long-term observation of comets, there is a small probability that comets alter their primary class, however they change frequently their subclass. This result supports the idea that the two major classes of comets are from different sources.

In particular, there are two large reservoirs for comets; the scattered disk (usually described as Kuiper Belt) on the far side of Neptune, and the Oort cloud extending to distances of $\sim 10^{5}$ AU from the Sun. The origin of comets usually are argued by the formation of Oort cloud (spherical in shape) and the scattered disk (flattened). The nearlyisotropic comets are believed to originate in the Oort cloud. The orbit of stored comets in the Oort cloud are affected by the Galaxy, particularly the angular momentum of the orbit. This results in large disturbance in the inclination and the perihelion distance of the comet. The origin of other category, ecliptic comets, was first thought to originate in the Oort cloud, but the low inclination of Jupiter-family comets ( a major population of ecliptic comets) suggest that they must have been generated from a flattened structure. The dynamically active region of the scattered disk causes the objects escape the disk with time. The bodies can either evolve outward to meet the Galactic tides or inward to encounter one of the outer planets e.g. Saturn, Uranus, and Neptune. In conclusion, the current models indicate that comets in both regions, the Oort cloud and the scattered disk 
formed in the planetary zone and were migrated by gravitational perturbations from the giant planets to their current locations (Levison and Dones, 2007).

\subsection{Space missions to asteroids and comets}

The recent spacecraft missions to asteroids and comets are tremendously effective to answer many scientific questions on composition, structure, and surface processes of asteroids and open a new view to the cometary nuclei world. Up to this point, the results of several flybys and few sample return missions are published. In the following, missions with imaging instruments to small bodies are described chronologically.

The first mission with imaging system started by two VEGA spacecraft to comet Halley in 1986 (the Soviet Union project). The VEGA 1 flew by comet at the distance of $8890 \mathrm{~km}$ and the VEGA2 within $8030 \mathrm{~km}$. The VEGAs images were helpful to recognize the comet's nucleus. but they were relatively noisy and farther from target in comparison with the images taken by the European Space Agency (ESA) mission, Giotto, in the same year (14 March 1986). The Giotto spacecraft approached the nucleus of Halley's comet at the distance of $596 \mathrm{~km}$. The spacecraft was equipped with the Halley Multicolor Camera (Keller et al., 1987) which, however, stopped working due to a dust particle impact around the time of closest approach (CA). The images before CA exhibit the nucleus shape to be elongated with a long axis of $\sim 15 \mathrm{~km}$ and a short axis of $\sim 7 \mathrm{~km}$. The nucleus of Halley with the average albedo of 0.04 showed a dark surface with the variety of features such as hills, and craters.

The next mission was the Galileo spacecraft which had two flybys at two asteroids (951) Gaspra and (243) Ida, on its way to Jupiter in 1991, and 1993. The images of Gaspra indicate that the object is of irregular shape (Figure 1.3). The albedo of 0.22 is typical for S-class asteroids. The surface is covered by more than 600 craters, many of them are fresh and young. In addition to the images of asteroid Ida, an unexpected result from the Galileo was the detection of the small satellite of asteroid Ida, Dactyl. The pair has similar photometric properties, but they are very different in their spectral characteristics (Veverka et al., 1996).

Later on, as the first mission completely assigned to asteroids, the NEAR (Near Earth Asteroid Rendezvous) spacecraft flew past asteroid (253) Mathilde in 1997 (Veverka et al., 1999) and reached at the end of its journey to asteroid (433) Eros (a near-earth asteroid) in 2001 (Cheng, 2002). The NEAR images which covered about 50\% of Mathilde's surface indicate that the object has five large craters (diameters between 20 and $33 \mathrm{~km}$ ). The geometric albedo of $0.047 \pm 0.005$ as well as the spectral properties of Mathilde are in agreement with $\mathrm{CM}$ carbonaceous chondrites spectra and confirm that the object belongs to the $\mathrm{C}$ type asteroid category. The Near Shoemaker spacecraft was orbiting asteroid Eros for about one year, and studied its target extensively. Eros is a $\mathrm{S}$ type asteroid with geometric albedo of $0.25 \pm 0.05$ which has the most elongated shape (Figure 1.3) among asteroids $\left(33 \times 10.2 \times 10.2 \mathrm{~km}^{3}\right)$. However, so far the surface of Eros is excessively cratered including the existence of boulders.

The comet 19P/Borrelly was the second comet after comet 1P/Halley that was imaged by NASA's Deep Space 1 (DS1) spacecraft on September 22, 2001 (Soderblom et al., 2004). The DS1 images suggest that the nucleus of the comet is dark with albedo variation 

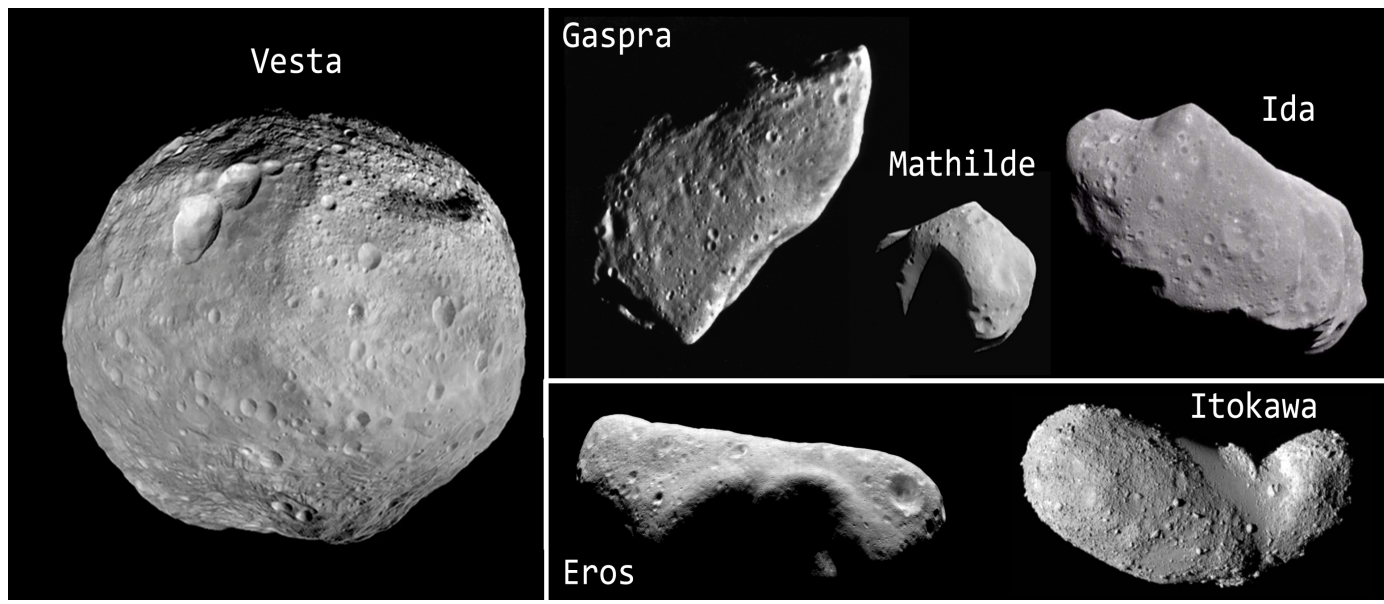

Figure 1.3: The six asteroids that have been visited by spacecraft: The main belt asteroids (951) Gaspra, (243)Ida, and (253) Mathilde were imaged during the flyby. The near-Earth asteroids (433) Eros and (25143) Itokawa, as imaged by a probe that orbited the targets. The asteroid (4) Vesta, one of the massive member of the asteroid belt, from the image taken by spacecraft that remain in orbit around the body more than one year.

over the surface (between 0.01 and 0.03 ). The surface of the nucleus is free of any impact crater (Figure 1.4).

NASA's Stardust spacecraft encountered comet 81P/Wild2 on 2 January 2004 (Brownlee et al., 2004). The Stardust images (Newburn et al., 2003) reveal a wide variety of morphological features on the surface of Wild2 including plenty of depressions, diverse small pinnacles, evidence of impact craters and a scarp with $2 \mathrm{~km}$ long (Figure 1.4). The nucleus of Wild2 is approximate of spherical shape with a diameter of $4 \mathrm{~km}$, different from the potato-shaped of the two other comets.

The Deep Impact (DI) spacecraft reached comet 9P/Tempel 1 on July 4th, 2005 (A'Hearn et al., 2005). The images from the DI mission (Hampton et al., 2005) show another different surface of a cometary nucleus with a number of circular features and smooth areas. The nucleus of Tempel 1 with an average diameter of $6.0 \mathrm{~km}$ is not roundish as Wild2's nucleus. The albedo and color variation change over the surface of Temple 1 homogeneously.

In the same year (September 2005), the Japanese mission, Hayabusa, was dedicated to small bodies (Fujiwara et al., 2006). The Hayabusa spacecraft moved in orbit around Near-Earth asteroid, (25143) Itokawa and collected material from its surface by accomplishing two touchdowns. Itokawa is a peanut-shaped (the longest dimension $\sim 700 \mathrm{~m}$ ) with an extremely rough surface. The surface is covered by boulders with of sizes up to $50 \mathrm{~m}$. No indication of craters are found on the surface. A smooth and featureless area is dominant in the center of the body surface (Figure 1.4).

In November 2010, the DI mission, extended to Deep Impact eXtended Investigation (DIXI), had another fly-by at a comet. The hyperactive comet 103P/Hartley2 was the target for the DIXI mission (A'Hearn et al., 2011). The nucleus of Hartley2 is small and may be formed by two lobe shaped components connected through smooth area. No depression was seen over the surface of the nucleus (Figure 1.4).

The two other missions which are devoted to the small body explorations are Rosetta 


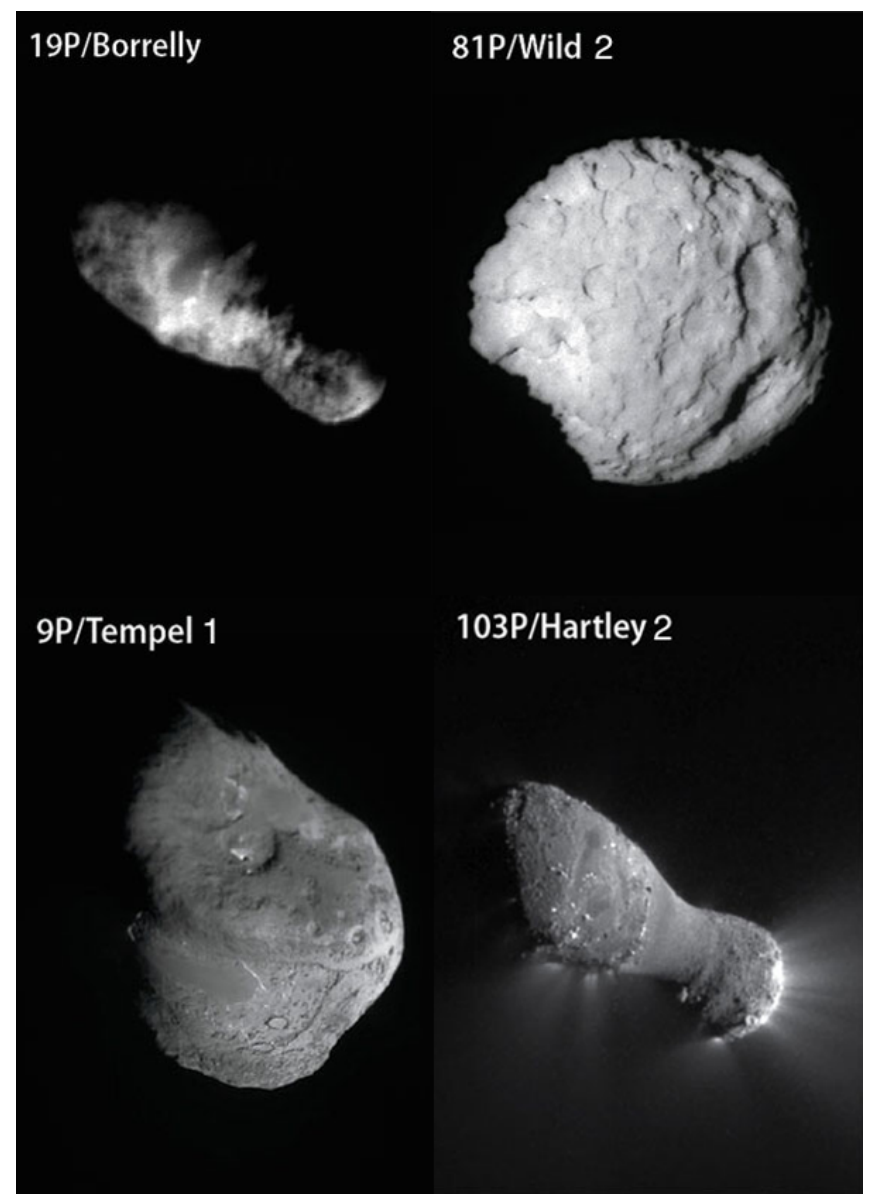

Figure 1.4: Images of four cometary nuclei acquired during the spacecraft flyby: comet 19P/Borrelly (Courtesy of NASA/JPL.), comet 81P/Wild 2 (Courtesy of NASA and the Stardust Mission Team.), comet 9P/Tempel 1 (Courtesy of NASA/JPL-Caltech/UMD.), comet 103P/Hartley (Courtesy of NASA/JPL-Caltech/UMD.).

(Schulz et al., 2009) by ESA and Dawn by NASA. Up to this time, they have performed part of their tasks. The details about the Rosetta mission and the OSIRIS (Optical, Spectroscopic, and Infrared Remote Imaging System) camera system onboard the spacecraft are presented in chapter 3 . The Rosetta Spacecraft, on its way to the main mission target, comet 67P/Churyumov-Gerasimenko in 2014, it had two close approaches one at asteroid (2867) Steins on 5 September 2008 (Accomazzo et al., 2010 Schulz, 2010) and one at asteroid (21) Lutetia on 10 July 2010 (Schulz et al., 2012). Steins is a small E type asteroid with shape similar to a diamond. The average visual albedo of this body is 0.35 . Close to the northern pole of Steins, a big crater ( $\sim 2 \mathrm{~km}$ in diameter) is found, compared with the small size of asteroid $\left(6.67 \times 5.81 \times 4.47 \mathrm{~km}^{3}\right)$. While the whole surface of Steins is covered with craters ( 23 craters in total), a chain of about seven craters, sometimes seen on large moons, is the stunning structure on the surface (Keller et al., 2010). Another target of Rosetta, asteroid Lutetia, is a large asteroid with diameter of $98 \pm 2 \mathrm{~km}$ and with an high bulk density of $3.4 \pm 0.3 \mathrm{~g} / \mathrm{cm}^{3}$. The spectral type of the asteroid is still unknown, mostly because of its complex surface. A wide range of features were identified on the surface of Lutetia such as grooves, boulders, lineaments, and craters. The most remarkable feature 
is close to the north pole of asteroid specified by name of the North Polar Crater Cluster (NPCC), an area which contains three large craters with the diameters of 14, 21 and 33 km (Sierks et al., 2011).

The Dawn spacecraft completed its mission at asteroid (4) Vesta between May 2010 to August 2011 and will reach to the next target, the dwarf planet, Ceres in April 2015. The surface of asteroid Vesta shows elaborate topography on different scales. The giant basin with a diameter of $500 \pm 20 \mathrm{~km}$ at the south pole of Vesta seen as the evidence that the Howardite-Eucrite-Diogenite (HED) meteorites are indeed coming from Vesta (Russell et al., 2012).

\subsection{Space weathering of asteroid surfaces}

The term space weathering is used for the physical processes on the surface of airless bodies that can modify the apparent characteristics such as the optical, structural, and chemical properties of the surface. Clark et al. (2002) reviewed what is currently known about space weathering on asteroids. Several physical processes are believed to cause space weathering such as the solar wind, micro-meteoroid bombardment and cosmic-rays.

Due to space weathering effects, the interpretation of remote sensing surface data as well as sample measurement is critical and it is difficult to link the bulk material properties of these bodies to their prime sources such as meteorites. Space weathering effects on asteroids are generally studied by remote sensing tools because no real soil sample is available from the surface of these objects. The topic of space weathering on asteroids arose when no clear connection was found (spectral mismatch) between the most abundant type of meteorites, ordinary chondrites, and the largest class of asteroids, the S-type asteroids. As argued by Gaffey (2010), a feasible explanation for the spectral incompatibility is that space weathering altered the spectral parameters such as the band depth, the albedo and the red slope of body (Figure 1.5).

The level of alteration is different for the various classes of asteroids and it depends on the physical and mineralogical properties of the bodies (Clark et al., 2002). Among asteroids, the C-type asteroids shows the smallest degree of alteration; one example is the surface of Mathilde as a C-type asteroid, which shows no albedo and color variation across the surface as well as no spectral variation near crater walls. This general behavior indicates that the objects contain dark and relatively opaque material, which show less space weathering than those consisting of bright, relatively transparent material. In the case of meteorites, experiments reveal that olivine is more sensitive to weathering than pyroxene (Hapke, 2001).

The connection between geomorphological features and optical variations is another important factor to study the space weathering of asteroid surfaces (Clark et al., 2002). However, this effect appears differently and depending on the distinct weathering environment. For instance, asteroid Eros which is in near-Earth orbit, exhibits entirely different color unit association compared to the main belt asteroids of Gaspra and Ida. On Eros, the albedo variations are high, color variegation is low, and bright material is found close to steep crater walls. On Gaspra and Ida, albedo variations are low, color variations are high and there are color features associated with fresh craters on ridges. 


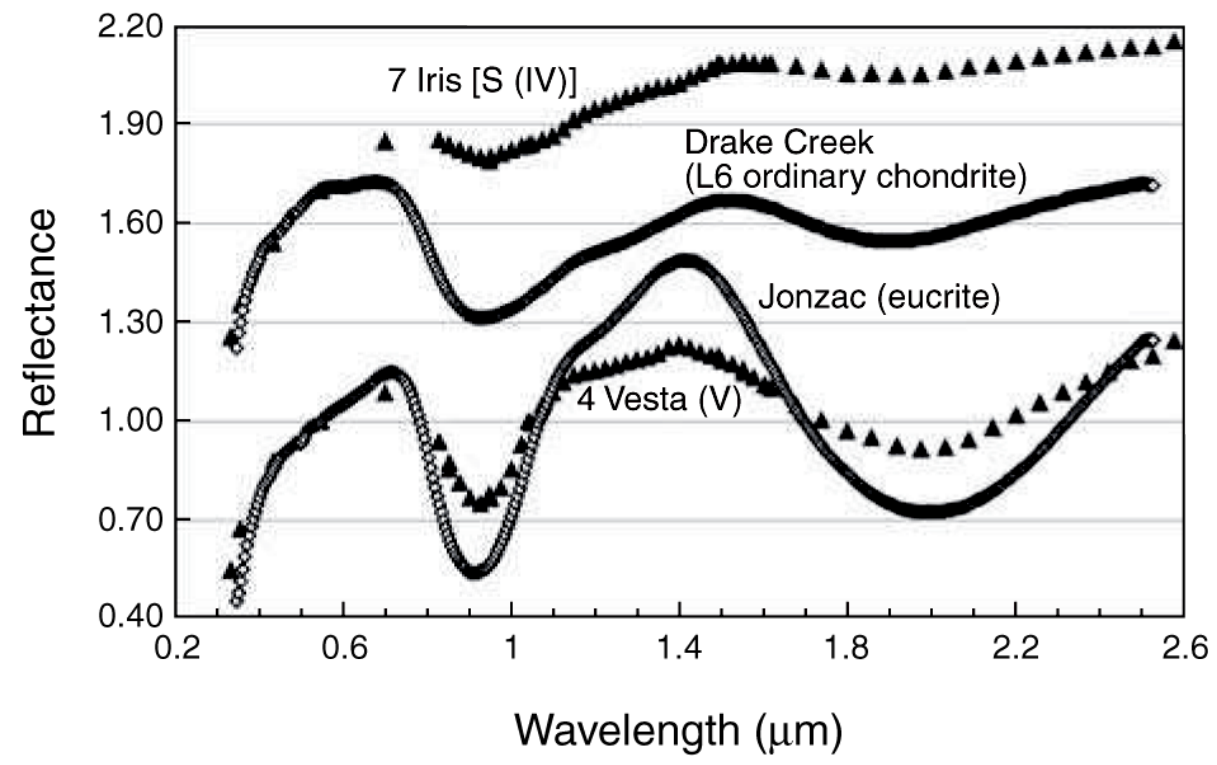

Figure 1.5: The reflectance spectra of two asteroids, (4) Vesta and (7) Iris, together with the spectra of meteorites are believed to be their meteorite analogues, as an example of space weathering. Space weathering process can cause the spectral mismatches.

\subsection{The reflectance analysis of small bodies}

One way of characterizing the physical properties of small bodies is the analysis of the reflected light from the surface. The reflected light from small bodies in the solar system particularly asteroids and comets can be measured from Earth-based and space-based observations. The work of Müller (1893) was the start of this kind of investigation into the ground-based photometry of small bodies. His work was mainly focused on the study the brightness changes with respect to phase angle, i.e. of the phase curve of some asteroids. For asteroids, it is possible to study the objects by observing the integral brightness. Until now, many asteroids are explored by constructing the phase curves from telescopic data. The disk-integrated phase function of asteroids from earth, however, is obtainable for very restricted geometries only. Nonetheless it returns valuable information about the physical properties of their surfaces. For comets, it is not practicable to measure the integrated brightness of the nuclei owing to the fact that they are barely perceptible at aphelion and at perihelion difficult to be isolated from the surrounding gas and dust of comae.

The physical interpretation of integrated radiance was of great interest in the 1960s. At the beginning, the primary tool was the Lommel-Seeliger (LS) theory which was extended later by Hapke (1963b, 1966) and Irvine (1966). More advanced light scattering models are developed by Hapke (1981, 1984, 1986) and Lumme and Bowell (1981) to better describe the characteristics of the actual regoliths. The developed models comprise effects of the microstructure, macroscopic roughness and multiple scattering.

There are two important findings from the disk-integrated reflectance analysis. One of them is that the phase curves of two major asteroid types, the typical S-type and Ctype are different (Bowell and Lumme, 1979). The other one was the recognition of the sharp increase in brightness of most of the asteroids towards zero phase angle, known as 
opposition effect (Gehrels, 1956).

The observation of asteroids from space gives the possibility to study these objects over a wider range of phase angles, while for the comets it becomes possible, for the first time, to build the phase function of cometary nuclei. Another considerable achievement comes with spatially resolved images from the asteroids and comets. Indeed, the application of light scattering models is more accurate and less ambiguous by using disk-resolved images. Both the Hapke and the Lumme \& Bowell photometric models are applicable to disk-integrated and disk-resolved data. The modeling leads to remotely identify the light scattering properties of separate regolith grains or aggregates for instance the angular pattern or transparency of particles. Other results from photometric modeling include constraining the geological properties of the regolith or the large-scale roughness of the surface.

Among the light scattering models, the Hapke model is widely applied to planetary surfaces. The Hapke function is reliable enough to derive the light scattering behavior of the surfaces and the Hapke light scattering parameters are well-suited to planetary cartography (the photometric correction of planetary images for mapping). Hapke's theory has been collected in the book Hapke (1993). Hapke's book is a primary reference in the reflectance analysis of planetary surfaces and being upgraded and scrutinize by numerical, experimental and spacecraft data. Hapke's modeling has been performed on disk-integrated and disk-resolved data of four cometary nuclei, comet 19P/Borrelly Li et al. (2013a), comet 81P/Wild2 (Li et al., 2009) and several asteroids, asteroid Gaspra (Helfenstein et al., 1994), asteroid Ida Helfenstein et al. 1996, asteroid Eros (Li et al., 2004), asteroid Itokawa (Kitazato et al., 2008), asteroid Steins (Spjuth et al., 2012), and asteroid Vesta (Li et al., 2013c) so far visited by spacecraft. The Hapke light scattering theory is reviewed in chapter 2 together with a short description of other light scattering models.

The physical properties of small bodies can be further constrained by laboratory studies. The reflectance of samples are measured in the laboratory to either verify the physical model or to expand the material data set. Several experimental studies attempted to investigate the relation between the retrieved Hapke parameters and selected samples covering a large range of photometric geometries. For instance, Shepard and Helfenstein (2007) and Shkuratov et al. (2012) verified the relation between retrieved Hapke parameters and soil samples. McGuire and Hapke (1995) and Cord et al. (2003) investigated the light scattering of particles with diverse sizes, types and shapes as well as. More recently, Beck et al. (2012) measured the reflectance from powder samples of meteorites expected to be representative of asteroids. In chapter 6 , the photometric analysis results from diskresolved data as well as the result of laboratory measurements will be discussed in detail and compared with the results from this thesis for asteroids Steins and Lutetia. 


\subsection{Motivation of the thesis}

The work presented in this thesis intends to analyze disk-integrated and disk-resolved reflectance from the surface of small atmosphereless bodies using the photometric model as a tool to constrain the physical properties of asteroids and cometary nuclei. In particular, we focus on the surface reflectance analysis of asteroid Lutetia and Steins using multiband, visible wavelength images obtained by OSIRIS onboard Rosetta during the flybys. We aim:

- to explore the light scattering parameters of the surface which are related to optical and structural properties of the asteroid surfaces. This allows to put Steins and Lutetia in context for surface characterization in reflected light of other solar system bodies,

- to study photometric variations across the surface, which helps to explore intrinsic uniformity and diversity as indicator of possible surface processing and,

- to provide values as initial inputs for photoclinometric (shape-from-shading) techniques used for extracting topography from spacecraft images. This means to give adequate baseline for surface DTMs using full image information. 


\section{Light reflectance theory}

\subsection{General terminology}

In remote sensing, there are some concepts to utilize scattering models for deriving physical properties of small body surfaces. These quantities are mainly involved to explain the interaction of electromagnetic radiation with particles in the frame of reflected light from surface layers of atmospherless celestial bodies. In this section, the description of these terms defined by Hapke, 1993 is outlined in brief.

\subsubsection{Scattering Surface}

The medium to which the incident light enters and of which scattering happens, is considered as a surface. To study light scattering from surfaces, the scattering surface is defined as a volume containing particles and interconnected voids. Therefore, the term particulate surface used for atmospherless bodies e.g., asteroid surfaces, are consist of heterogeneous particles assumed by Hapke theory to be large compared with the wavelength.

\subsubsection{Geometrical angles}

The radiation geometry mostly encountered in the planetary remote sensing is illustrated in Figure 2.1. A scattering medium is illuminated by the Sun. The z-axis shows the normal $\mathbf{N}$ to the surface, and the angle between the Sun and the surface normal $(\mathbf{N})$ is called incidence angle, $i$. After the interaction between the incident light and the medium, the emergent rays are observed by a detector at an angle between observer and surface normal $\mathbf{N}$, that is called emission angle, $e$. The plane consisting of the incident ray and $\mathbf{N}$ is the plane of incidence, and the one containing the emerging ray and $\mathbf{N}$ is the plane of emergence. The planes of incidence and emergence are inclined by an angle defined as the azimuth angle, $\psi$. Lastly, the angle forms between incident ray and emergent ray specified by phase angle, $\alpha$. The relation between the four angles i,e, $\alpha, \psi$ can be given as

$$
\cos \alpha=\cos i \cos e+\sin i \sin e \cos \psi
$$

where $0 \leq \psi \leq \pi$. The cosines of $i$ and $e$ are conventionally replaced by two symbols, $\mu_{0}$ and $\mu$, respectively. 


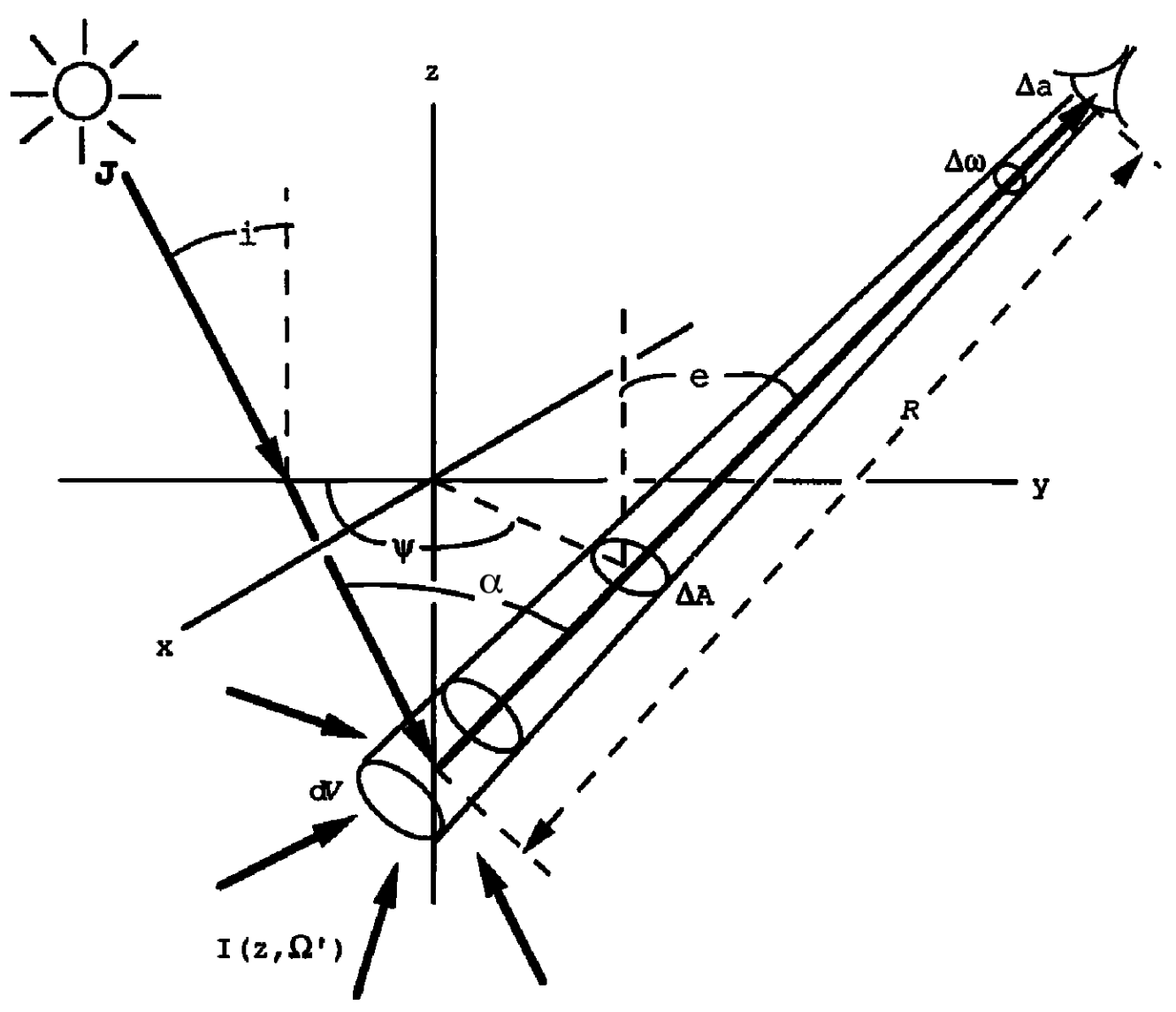

Figure 2.1: The scheme of scattering angles during the interaction of light from inside a particulate medium. The $x-y$ plane depicts the theoritical surface of the medium (taken from figure 8.4 in Hapke, 1993).

\subsubsection{Reflectance}

The amount of incident light scattered or reflected by a rough medium is described by the term reflectance. In accordance with geometry, there are diverse types of reflectance. To avoid any ambiguity, the word reflectance can be preceded by two adjectives. The first adjective illustrates the degree of collimation of the source and the second one that of the detector.

Well-established adjectives are directional, conical, or hemispherical. In case of duplicate adjectives, the prefix $b i$ - is used. For instance, the directional-hemispherical reflectance refers to the total fraction of light reflected into the upper hemisphere when the surface is illuminated by a collimated source from above. Another instance, the bidirectional reflectance $r(i, e, \alpha)$, refers to the fraction of light scattered into direction $e$ when the surface is illuminated by collimated incident light in direction $i$. In Hapke's theory, as he described in Hapke (1993), the bidirectional reflectance is generally used to determine reflectance from the surface; however, this term is physically ideal. In fact, what we are measuring is a biconical reflectance since both collimated source and detector have a finite amount of solid angle. On the other hand, for most cases of remote sensing, the solid 
angle as viewed from the object is very small for both source and detector. Therefore, although the bidirectional reflectance is not completely accurate, it is a notable simplification in theoretical analysis. The general expression for the bidirectional reflectance at wavelength $\lambda$ is given by

$$
r\left(\mu_{0}, \mu, \alpha, \lambda\right)=I\left(\mu_{0}, \mu, \alpha, \lambda\right) / J(\lambda)
$$

where $I$ is the intensity in $\mathrm{W} \mathrm{m}^{-2} \mu \mathrm{m} \mathrm{sr}^{-1}$ and $J$ is the solar flux in $\mathrm{W} \mathrm{m}^{-2} \mu \mathrm{m}$, determined at the distance between Sun and object. Another expression related to the bidirectional reflectance and widely used as a dimensionless quantity is the radiance factor. The radiance factor, denoted by $\mathrm{I} / \mathrm{F}$, is specified as

$$
I / F=\pi r\left(\mu_{0}, \mu, \alpha, \lambda\right)
$$

where $I$ is the reflected intensity from the illuminated surface, and $\pi \mathrm{F}$ is the incoming solar flux onto a perfectly diffuse surface, illuminated at $i=0^{\circ}$.

\subsection{Radiative Transfer Equation}

The expression that is widely used in planetary science to illustrate the series of emission, absorption, and scattering processes for an electromagnetic wave interfacing with planetary regolith and the atmosphere is known as the equation of radiative transfer. The equation of radiative transfer can be derived by considering light propagation in a cylinder of volume $d s d A$. Therefore, the change of intensity $I(s, \Omega)$ at a point $s$, traversing in the direction of $\Omega$ can be described by,

$$
\frac{\partial I(s, \Omega)}{\partial s}=-E(s, \Omega) I(s, \Omega)+\frac{1}{4 \pi} \int_{4 \pi} I\left(s, \Omega^{\prime}\right) G\left(s, \Omega^{\prime}, \Omega\right) d \Omega^{\prime}+F(s, \Omega)
$$

where $E(s, \Omega)$ and $G\left(s, \Omega, \Omega^{\prime}\right)$ are the extinction and volume angular scattering coefficients, respectively. $E(s, \Omega)$ contains the absorption and scattering coefficients which refer to a decrease in the energy of radiance. The scattering process can cause an increment in the intensity as well; $G\left(s, \Omega, \Omega^{\prime}\right)$ represents the probable event that the photon traveling in direction $\Omega^{\prime}$ can be scattered into direction $\Omega$. The contribution of emission process is specified by the volume emission coefficient, $F(s, \Omega)$. Two main processes are usually considered as part of the emission coefficient in the light scattering of objects, i.e. the single scattering and thermal emission.

\subsubsection{Single-particle light scattering model}

The properties of scattered light can be understood in terms of single-particle scattering. Two quantities, i.e. single-scattering albedo and phase function of the particles, are used in general definition of light scattering from a single particle.

The single-scattering albedo, denoted by $\omega$, is defined as the ratio of the sum of power scattered to the sum of power abolished from the incident irradiation, J.

The single-particle phase function, $p(\alpha)$, represents the angular distribution of the reflected brightness $I\left(\Omega, \Omega_{0}\right)$ with respect to the phase angle, $\alpha$. Thus, $p(\alpha)$ is defined by,

$$
I(\Omega)=\omega J\left(\Omega_{0}\right) \frac{p(\alpha)}{4 \pi}
$$


where $J$ is the solar flux into direction $\Omega_{0}$. For isotropic-scattering particles, $p(\alpha)$ is equal to 1 ; the normalization formula of $p(\alpha)$ is given by, $\int_{4 \pi} p(\alpha) d \Omega=4 \pi$.

The light reflected by a uniform spherical particle is modeled following Mie scattering theory. The Mie theory includes the exact solution for the interaction of electromagnetic radiation with an isolated perfectly spherical particle. The fundamental parameter for the scattering of single spherical particles is called the size parameter, $\mathrm{X}$, defined as the ratio of particle size to the wavelength of incident radiance, is shown in the form of $X=\pi D / \lambda$ (where $\mathrm{D}$ is the diameter of particle). The Mie theory results in different approximations based on the comparison of the size parameter with unity. When $X \ll 1$, the particle size is much smaller than the wavelength and the scattered light is proportional to $1 / \lambda^{4}$. The scattering is known as Rayleigh scattering. Assuming that the incident radiance is unpolarized, the particle phase function is given by,

$$
p(\alpha)=\frac{3}{4}\left(1+\cos ^{2} \alpha\right)
$$

If $X \approx 1$, the particle size is the same level of wavelength. In this region, also known as Resonance region, the behavior of the single particle phase function involves many different and intricate aspects from case to case. For $X \gg 1$, the size of particle is much larger in comparison to the wavelength and the scattering is explained by the geometricoptic scattering, considering diffraction at large phase angles.

The light scattering model of a perfect, uniform, spherical particle is applicable in the very rare cases in nature. There is no exact expression to describe the scattering behavior of irregular particles; alternatively, approximate expressions based on the empirical models are used. There are two empirical single-particle phase functions which are widely utilized, the Legendre polynomial series and the Henyey-Greenstein function.

The Legendre polynomials is capable to represent the single-particle phase function for irregular particles,

$$
p(\alpha)=\sum_{j=0}^{\infty} b_{j} P_{j}(\alpha)
$$

where the coefficients $b_{j}$ are constants, and $P_{j}(\alpha)$ are Legendre polynomials of order $j$. If the divergences from isotropic scattering are not that much, the first- and second- order expansions of Legendre polynomial are competent.

Another empirical phase function introduced by Henyey and Greenstein (1941) and called single-term Henyey-Greenstein $(H G)$ phase function, is given by,

$$
p(\alpha)=\frac{1-g^{2}}{\left(1+2 g \cos \alpha+g^{2}\right)^{3 / 2}}
$$

where $g$ is the cosine asymmetry factor to describe the angular pattern of scattering for irregular particles. The parameter $\mathrm{g}$ varies from back-scattering $(\mathrm{g}<0)$ through isotropic scattering $(\mathrm{g}=0)$ to forward scattering $(\mathrm{g}>0)$ in the range from -1 to 1 . The scattering structure can be described better by a double-term Henyey-Greenstein phase function. The two-term HG function (2HG) separates the back-scattering and forward scattering lobes. The form introduced by McGuire and Hapke (1995) is widely used,

$$
P(\alpha)=\frac{1+c}{2} \frac{1-b^{2}}{1-2 b \cos \alpha+b^{2}}+\frac{1-c}{2} \frac{1-b^{2}}{1+2 b \cos \alpha+b^{2}}
$$


With the coefficient $\mathrm{b}$ in the range from 0 to 1 and the coefficient $\mathrm{c}$ with no constraint, apart from the general constraint of $p(\alpha)$. The first part of Equation 2.9 is related to the backward-scattering lobe, and the second part to the forward-scattering lobe. The width and height of the lobes are represented by the parameters $b$ and $c$, respectively.

The physical meaning of the HG parameters $b$ and $c$ are studied by McGuire and Hapke (1995) through an experimental analysis. Their study is done for diverse particles with different compositions and shapes, but approximately of the same size fulfilling the criteria to be large compared to the wavelength. The $\mathrm{b}$ and $\mathrm{c}$ parameters resulting from the experiment are plotted versus each other (Figure 2.2). It is evident that all the particles accumulate in a L-shaped area. The Figure 2.2 is turned to Figure 2.3, in order to outline a general scheme of HG parameters behavior. The position in the HG plot suggests not only the angular pattern of light scattering, but also the physical characteristics of the particles. Near the lower right branch of the L diagram, the smooth, clear and spherical particles are located which also correspond to the strong forward scatters. Toward the center, the shape of particle is irregular which is apparent in the values of $b$ (decreasing) and c (increasing). Further irregularity in the shape of the particle leads to $b \sim 0.25$, indicating that the rough, irregular particles have approximately constant $b$ and increasing c. Slightly farther toward the end of the L, the high back-scattering particles such as dielectrics and metals are found with $c \sim 1.2$.

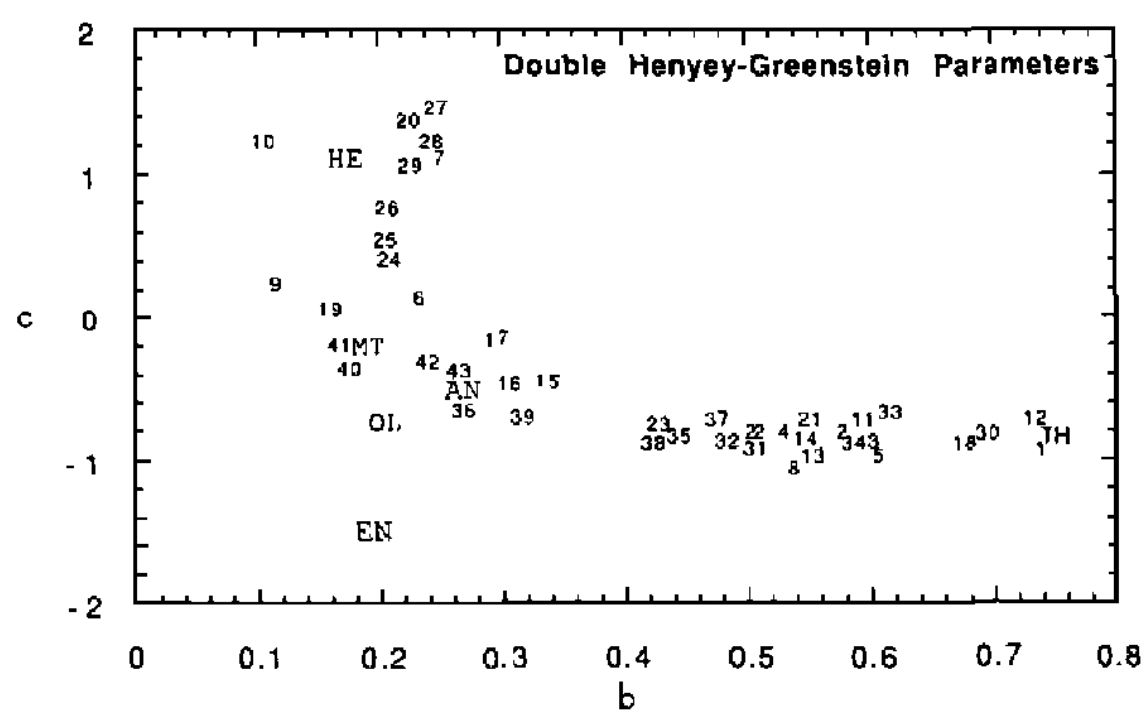

Figure 2.2: The plot of HG parameters $\mathrm{b}$ and $\mathrm{c}$ against each other, adapted from figure 16a in McGuire and Hapke (1995), showing the results of their study for a collection of irregular particles of various types. The numbers are related to the numbers attributed to the particles used in the experiment. 


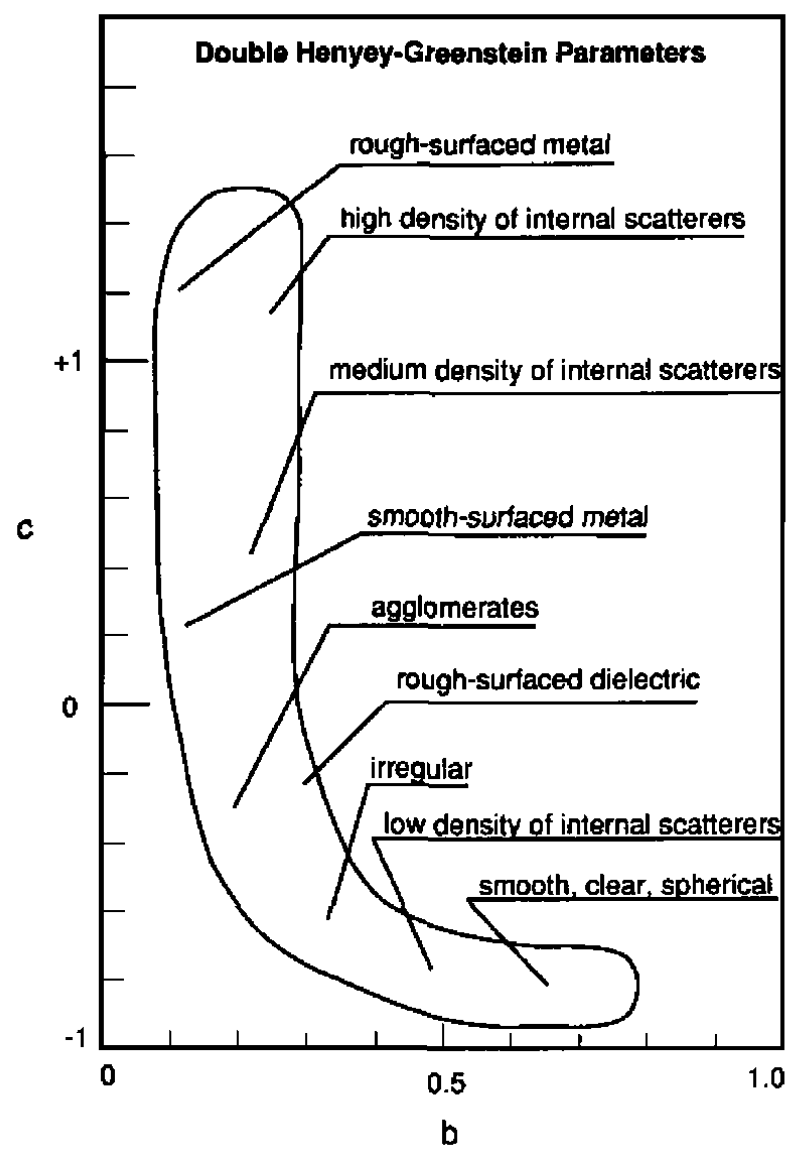

Figure 2.3: $2 \mathrm{HG}$ parameters diagram, adapted from figure 16b in McGuire and Hapke (1995), illustrates the data points in Figure 2.2 in a practical format.

\subsubsection{Hapke Scattering Model}

Hapke has developed his theory in several publications (Hapke, 1981, 1984, 2002). In the following, a brief outline is prepared for the context of light scattering of planetary and small body surfaces. The Hapke light scattering theory is derived from radiative transfer equation solution for a semi-infinite particulate surface. To derive the Hapke equation, a volume element $d V=R^{2} \Delta \omega d R$ can be assumed within an angular element $\Delta \omega$ at an elevation $z$ in the medium and a distance $R$ from the detector. The beam $I\left(z, \Omega^{\prime}\right) d \Omega^{\prime}$ traveling within solid angle $d \Omega^{\prime}$ in direction $\Omega^{\prime}$ radiates the volume element which cause an amount of power is scattered in $d V$ into unit solid angle about the direction $\Omega$ between $d V$ and the detector by particles.

$$
\frac{d V}{4 \pi} \int_{4 \pi} G\left(z, \Omega^{\prime}, \Omega\right) I\left(z, \Omega^{\prime}\right) d \Omega^{\prime}
$$

where $G\left(z, \Omega^{\prime}, \Omega\right)$ is the volume angular scattering coefficient. Additionally, an amount of emitted light from $d V$ reaches the detector,

$$
F(z, \Omega) d V
$$


where $F(z, \Omega)$ is the volume emission coefficient. The scattered and emitted radiance is weakened because of extinction processes by a factor $e^{-\tau / \mu}$. The optical depth, $\tau$, is a dimensionless unit to describe the fractional radiance is reduced by extinction during its path through a medium. The $\mu$ is equivalent to $\cos e$, where e is the angle between the emitted radiance and the vertical. Therefore, the power can be detected from the volume element $d V$ is

$$
d P_{D}=\left[\frac{1}{4 \pi} \int_{4 \pi} G\left(z, \Omega, \Omega^{\prime}\right) I\left(z, \Omega^{\prime}\right) d \Omega^{\prime}+F(z, \Omega)\right] d V \frac{\Delta a}{R^{2}} e^{-\tau / \mu}
$$

where the solid angle is $\triangle a / R^{2}$ as observed from $d V$. The Equation 2.12 can be simplified by introducing three ratios in the following; Firstly, the volume single scattering is given by,

$$
\omega(z)=S(z) / E(z)
$$

where $s(z)$ is the volume scattering coefficient and $\mathrm{E}(\mathrm{z})$ is the volume extinction coefficient. Another ratios is the volume phase function,

$$
p\left(z, \Omega^{\prime}, \Omega\right)=G\left(z, \Omega^{\prime}, \Omega\right) / S(z)
$$

and lastly, regarding $\tau$ can be substituted for $d z=\mu d R$. The source function can be defined as,

$$
\mathcal{F}=\frac{F(z, \Omega)}{E(z)}=\frac{\omega(\tau)}{4 \pi} J p\left(\tau, \Omega^{\prime}, \Omega\right) e^{-\tau / \mu_{0}}
$$

Subsequently,

$$
d P_{D}=-\Delta \omega \Delta a\left[\frac{\omega(\tau)}{4 \pi} \int_{4 \pi} p\left(\tau, \Omega^{\prime}, \Omega\right) I\left(\tau, \Omega^{\prime}\right) d \Omega^{\prime}+\mathcal{F}(\tau, \Omega)\right] e^{-\tau / \mu} \frac{d \tau}{\mu}
$$

The total light recovered by the detector (power per unit area per unit solid angle) can characterized by integrating $d P_{D}$ over all volume elements within $\Delta \omega$ between $z=-\infty$ and $+\infty$, or, equivalently, between $\tau=\infty$ and 0 . Thus,

$$
\begin{aligned}
I_{D} & =\frac{1}{\Delta \omega \Delta a} \int_{z=-\infty}^{\infty} d P_{D} \\
& =\int_{0}^{+\infty}\left[\frac{\omega(\tau)}{4 \pi} \int_{4 \pi} p\left(\tau, \Omega^{\prime}, \Omega\right) I\left(\tau, \Omega^{\prime}, \Omega\right) d \Omega^{\prime}+\mathcal{F}(\tau, \Omega)\right] e^{-\tau / \mu} \frac{d \tau}{\mu} .
\end{aligned}
$$

The first part of resultant equation embodies multiple scattering term. The second part is related to the single scattering and includes the source function $\mathcal{F}(\tau, \Omega)$.

\subsubsection{Single Scattering}

The radiance due to single scattering $\left(I_{D s}\right)$ is determined by

$$
I_{D s}=J \frac{1}{4 \pi} \frac{1}{\mu} \int_{0}^{+\infty} \omega(\tau) p(\tau, \alpha) e^{-\left(1 / \mu_{0}+1 / \mu\right)} d \tau
$$


Assuming $\omega$ and $\mathrm{p}$ are not function of the optical depth, $\tau$. The integral is evaluated as,

$$
I_{D s}=J \frac{\omega}{4 \pi} \frac{\mu_{0}}{\mu_{0}+\mu} p(\alpha)
$$

In the case of $p(\alpha)=1$, i.e. the isotropic scattering, Equation 2.19 is called LommelSeeliger Law. This scattering law excludes non-isotropic scatterers as well as multiple scattering processes. However, it is capable of describing the behavior of the light scattered by dark bodies, such as the Moon and Mercury.

\subsubsection{Multiple scattering}

It is not easy to include the multiple scattering part in the bidirectional reflectance model because solving the integral for the more complex scattering term is impossible. However, by considering a semi-infinite medium which is only composed of isotropic scatters, a solution becomes possible. Hence, the rigorous expression can be obtained either using the two-stream solution with collimated source (approximate approach) or the method of embedded invariance (exact solution). The two methods result in two solutions with identical form,

$$
r(i, e, \alpha)=\frac{\omega}{4 p i} \frac{\mu_{0}}{\mu_{0}+\mu} H\left(\mu_{0}\right) H(\mu)
$$

In the embedded invariance solution, $H(x)$ is given by the Ambartsumina-Chandrasekhar H-function (Chandrasekhar, 1960) that satisfies,

$$
H(x)=1+\frac{\omega}{2} x H(x) \int_{0}^{1} \frac{H\left(x^{\prime}\right)}{x+x^{\prime}} d x^{\prime}
$$

Where $\mathrm{x}$ substitutes with either $\mu_{0}$ or $\mu$ and $x^{\prime}$ is the integral variable. The integral is solvable by numerical computation. From the two-stream solution, $H(x)$ is approximated by

$$
H(x) \simeq \frac{1+2 x}{1+2 \gamma x}
$$

where $\gamma=\sqrt{1-\omega}$. A better form of the H-function is calculated by linearizing Equation 2.21 (Hapke, 2002). The approximation gives,

$$
H(x) \approx\left[1-\omega x\left(r_{0}+\frac{1-2 r_{0} x}{2} \ln \frac{1+x}{x}\right]^{-1}\right.
$$

where $r_{0}=(1-\gamma) /(1+\gamma)$. In this thesis, we apply the Hapke model to the disk-resolved data of asteroids, using the Equation 2.23 for the H-function.

It is more complicated to model the bidirectional reflectance of the medium of anisotropic scatterers. The most recent attempt to add anisotropic scattering particles in the solution for reflectance is explored by Hapke (2002). Here, the multiple scattering is given by,

$$
r_{m}=\frac{\omega}{4 \pi} \frac{\mu_{0}}{\mu_{0}+\mu} M\left(\mu_{0}, \mu\right)
$$


where

$$
M\left(\mu_{0}, \mu\right)=P\left(\mu_{0}\right)[H(\mu)-1]+P(\mu)\left[H\left(\mu_{0}\right)-1\right]+\mathcal{P}[H(\mu)-1]\left[H\left(\mu_{0}\right)-1\right]
$$

The terms $P\left(\mu_{0}\right), P(\mu)$, and $\mathcal{P}$ are defined as the average of particle angular scattering functions over the range of integration. The integrals are given as:

$$
\begin{aligned}
P\left(\mu_{0}\right) & =\frac{1}{2 \pi} \int_{e^{\prime}=\pi / 1}^{\pi} \int_{\varphi^{\prime}=0}^{2 \pi} p\left(\alpha^{\prime}\right) \sin e^{\prime} d e^{\prime} d \varphi^{\prime} \\
P(\mu) & =\frac{1}{2 \pi} \int_{i^{\prime}=\pi / 1}^{\pi} \int_{\varphi^{\prime}=0}^{2 \pi} p\left(\alpha^{\prime}\right) \sin i^{\prime} d i^{\prime} d \varphi^{\prime} \\
\mathcal{P} & =\frac{1}{(2 \pi)^{2}} \int_{i^{\prime}=0}^{\pi / 2} \int_{\varphi_{i}^{\prime}=0}^{2 \pi} \int_{e^{\prime}=0}^{\pi / 2} \int_{\varphi_{e}^{\prime}=0}^{2 \pi} p\left(\alpha^{\prime}\right) \sin e^{\prime} d e^{\prime} d \varphi_{e}^{\prime} \operatorname{sini}^{\prime} d i^{\prime} d \varphi_{i}^{\prime}
\end{aligned}
$$

The set of equations for the bidirectional reflectance model using the anisotropic multiple scattering are complicated. The practical application of this model is also inconvenient. As a matter of fact, most asteroids and cometary nuclei are dark objects and one can use the Hapke model for isotropic multiple scattering as a reasonable approach. For instance, the role of multiple scattering is studied by Li et al. (2013c) for asteroid (4) Vesta. They point out that using the anisotropic multiple scattering in the Hapke modeling does not show any significant improvement for their data interpretation. I have incorporated in my work the Hapke model for the medium of isotropic scatterers.

The contribution of two components of reflectance, single-scattering $\left(r_{s}\right)$ and multiple scattering from isotropic scatters $\left(r_{m}\right)$ leads to

$$
r(i, e, \alpha)=r_{s}+r_{m}=\frac{\omega}{4 \pi} \frac{\mu_{0}}{\mu_{0}+\mu}\left[p(\alpha)+H\left(\mu_{0}\right) H(\mu)-1\right]
$$

\subsubsection{Opposition Effect}

The Equation 2.29 applies to a medium with the particles scattering independently. Considering the particles are close together as in a powder or soil, Hapke included the effect of shadow hiding can cause a sudden increase at zero phase angle and contributes to the so called opposition effect or opposition surge. Photometric observations for many atmosphereless bodies of the solar system near the zero phase angle unveil a sharp increase in the reflectance. For asteroids, it usually happens between $\alpha$ of $5^{\circ}$ to $10^{\circ}$ (Figure 2.4). 


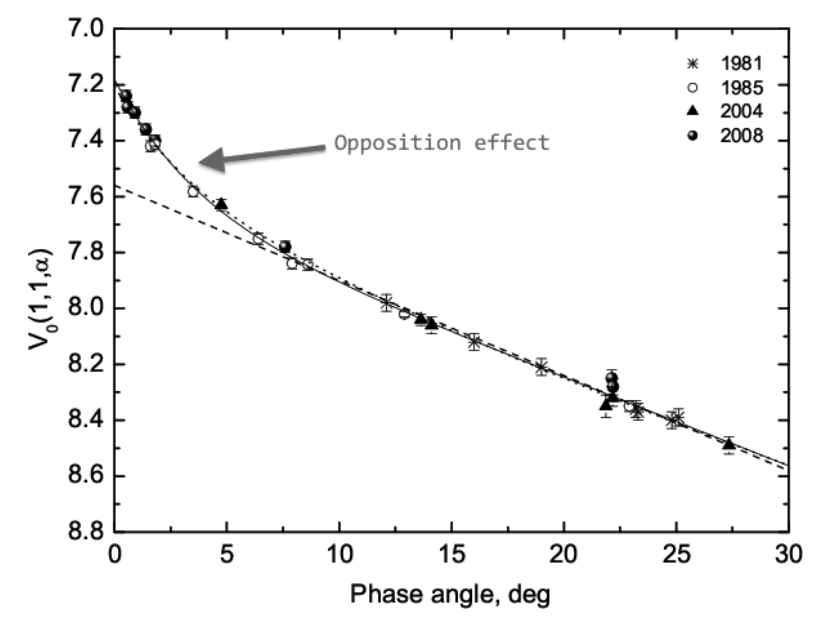

Figure 2.4: The solid curve depicts the sudden increase in the disk-integrated brightness of asteroid (21) Lutetia with respect to the phase angle reaches zero, adapted from figure. 3 in Belskaya et al. (2010)

There are two mechanisms proposed to explain this phenomena, as described by Hapke in Hapke (1993) and in the case of the Moon (Hapke et al., 1993). One of them by the name of shadow hiding opposition effect is illustrated that at opposition (almost $\alpha \simeq 0^{\circ}$ ) dispersed shadows by the particles are large compared with the wavelength, are hidden by the particles themselves (Figure 2.5).
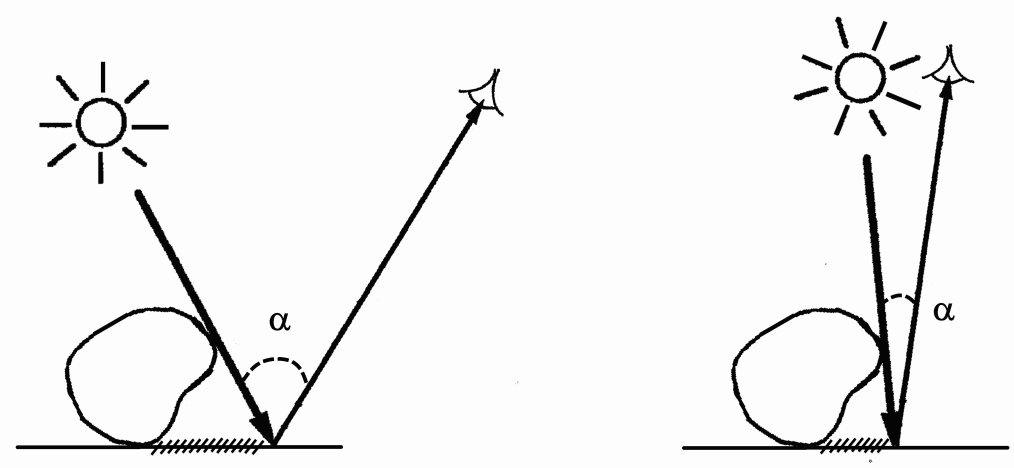

Figure 2.5: The illustration of SHOE

This mechanism has an effect exclusively on the single scattering part of the bidirectional reflectance, and is added analytically as a correction term to Equation 2.29. The form of the total bidirectional reflectance after including this effect is now given by

$$
r(i, e, \alpha)=\frac{\omega}{4 \pi} \frac{\mu_{0}}{\mu_{0}+\mu}\left\{\left[1+B_{S}(\alpha)\right] p(\alpha)+H\left(\mu_{0}\right) H(\mu)-1\right\}
$$

Where

$$
B_{S}(\alpha)=\frac{B_{S 0}}{1+\frac{1}{h_{S}} \tan (\alpha / 2)}
$$


In Equation 2.31, two parameters of $B_{S 0}$, and $h_{S}$ describe the amplitude and width of shadow hiding effect (SHOE), respectively. The amplitude of the SHOE can illustrate the level of transparency in regolith grains. The range of $B_{S 0}$ can vary from unity to nearly zero, where the upper limit means that the regolith is composed of perfectly opaque grains, and the lower limit represents transparent grains.

The angular width of SHOE is controlled by size distribution and paking density of grains. Hence, $h_{S}$ can be related to the porosity as Hapke presented in Hapke (1986), $\mathrm{h}_{S}=-$ $0.375 \ln (1-\phi)$, where $\phi$ is noted as the filling factor, the portion of area that is occupied by particles. After Hapke (2008), this relation is only correct to some extent, Helfenstein and Shepard (2011) modified it to

$$
h_{S}=-0.3102 \phi^{1 / 3} \ln \left(1-1.209 \phi^{2 / 3}\right)
$$

Another mechanism is so called the coherent backscattering opposition effect (CBOE) which is derived from the fact that partial waves traveling inside a medium is constituted of particles with comparable or smaller size than wavelength, may go through the same multiply scattered way in opposite directions. The rays of emerging light from the medium can combine coherently and be in phase at precisely zero phase angle.

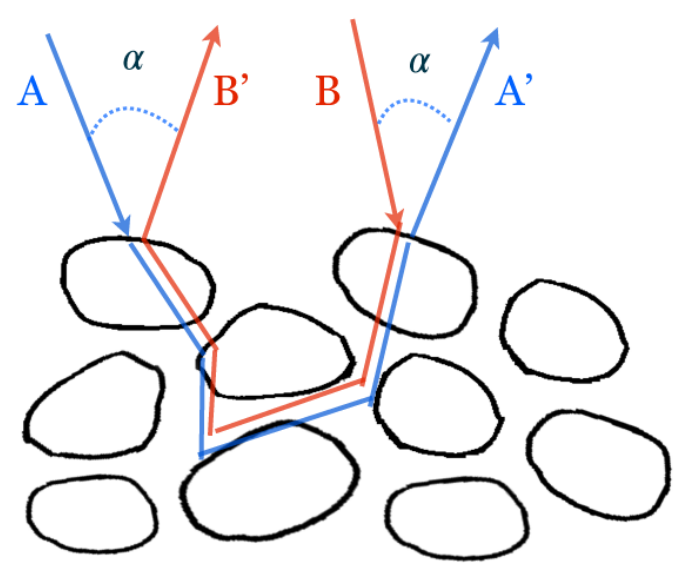

Figure 2.6: The illustration of $\mathrm{CBOE}$

The CBOE involves both single scattering and multiple scattering, besides it causes the negative branch in polarization phase curves. Hapke (2002) presented the bidirectional reflectance, taking into account $\mathrm{CBOE}$ by multiplying the whole function by

$$
1+B_{C}(\alpha)
$$

where $B_{C}(\alpha)$ is an approximate expression to describe the CBOE,

$$
B_{C}(\alpha)=B_{C 0} \frac{1+\frac{1-\exp \left[-\left(1 / h_{C}\right) \tan (\alpha / 2)\right]}{\left(1 / h_{C}\right) \tan (\alpha / 2)}}{2\left[1+\left(1 / h_{C}\right) \tan (\alpha / 2)\right]^{2}}
$$

In Equation 2.34, the two parameters of $B_{C 0}$ and $h_{C}$ illustrates the CBOE effect. The $B_{C 0}$ is the amplitude of the CBOE with the range of 0 to 1 . The width parameter of CBOE, $h_{C}$, is controlled by the optical properties of scattering medium,

$$
h_{C}=\lambda / 4 \pi \Lambda
$$


where the wavelength of incident light is $\lambda$, and the mean free path in the medium is, $\Lambda$. The mean free path $\Lambda$ can be seen as a parameter for the mean distance that a photon would travel in the medium before its direction is altered by a large angle (assuming 1 radian). $\Lambda$ is expressed as

$$
\Lambda=\left[n \sigma Q_{s}(1-\langle\cos \theta\rangle]^{-1}\right.
$$

where $n$ is the quantity of particles in unit volume, $\sigma$ is the mean cross section of the particles and $Q_{s}$ is the mean scattering efficiency of the particles. $\langle\cos \theta\rangle$ is the mean cosine of the scattering angle.

In Figure 2.7, the functions of $B_{S}(\alpha)$ and $B_{C}(\alpha)$ are plotted - instead of against $\alpha$ - versus the quantity of $z$. $z$ is a function of $\left(1 / h_{S}\right) \tan (\alpha / 2)$ for $B_{S}(\alpha)$, while $z=0.36\left(1 / h_{C}\right) \tan (\alpha / 2)$ for $B_{C}(\alpha)$. The comparison suggests that it would be almost impossible to distinguish $\mathrm{SHOH}$ from $\mathrm{CBOE}$. Hence, in many cases it may suffice to characterize the opposition effect by the SHOE only and considering the CBOE component, by limiting the opposition effect amplitude to two instead of one.

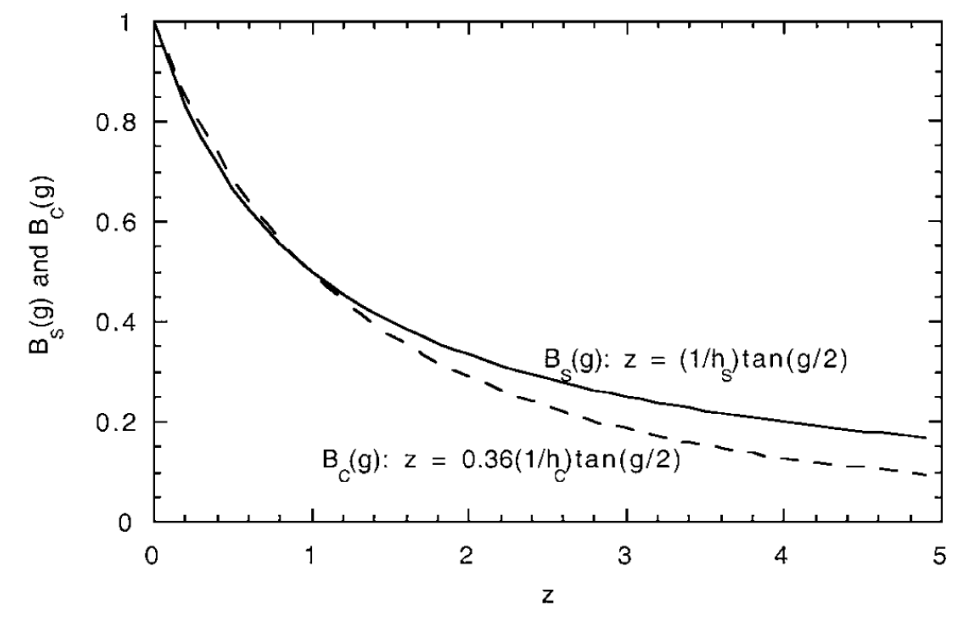

Figure 2.7: The comparison of $\operatorname{SHOE~} B_{s}(z)$ and CBOE $B_{c}(z)$ functions, taken from figure 9 in Hapke (2002).

\subsubsection{Macroscopic roughness}

The Equation 2.30 is valid for smooth surfaces on scales that are large in comparison with the size of the particles. This scenario easily applies for surfaces used in laboratory experiments. In the case of planetary regolith one has to consider also roughness on a large scale. Thus, in order to take this into account, Hapke (1984) presented a correction, the mean slope angle $(\bar{\theta})$, to include the effect of large-scale roughness. The surface is assumed to be formed by numerous small, unresolved facets tilted in different directions. The orientation of each individual facet is defined by its normal vector. A two dimensional distribution $a(\theta, \zeta) d \theta d \zeta$ is used to describe the normals, where $\theta$ is the zenith angle, and $\zeta$ is the azimuth angle. It is reasonable to assume that the slope distribution of surfaces is Gaussian for zenith angle and is independent of the azimuth angle. Hence, the roughness parameter of the surface is presented as follow,

$$
\tan \bar{\theta}=\frac{2}{\pi} \int_{0}^{\pi / 2} a(\theta) \tan (\theta) d \theta
$$


The two major aspects of macroscopic roughness would remodel the reflectance. One effect is due to shadows that cast since one part of the surface is obstructed by the other part to be either illuminated or be observed by the detector. This effect is characterized by the shadowing function, $\mathrm{S}(\mathrm{i}, \mathrm{e}, \alpha)$. The second one is the portions of the surface that have the tendency to be hidden when zenith angle starts increasing, this would provoke the effective surface which is tilted toward the source and detector. The tilted area of the surface is described by the effective incidence angle $i_{e}$, and the effective emission angles $e_{e}$.

In the following, the formalism of cosines, $\mu_{0 e}$ and $\mu_{e}$, as well as the shadowing function, $S$, are listed. The detail procedure of derivation is described in Hapke (1984).

If $i<e$,

$$
\begin{aligned}
\mu_{0 e} & \simeq \chi(\bar{\theta})\left[\cos i+\sin i \tan \bar{\theta} \frac{\cos \psi E_{2}(e)+\sin ^{2}(\psi / 2) E_{2}(i)}{2-E_{1}(e)-(\psi / \pi) E_{1}(i)}\right] \\
\mu_{e} & \simeq \chi(\bar{\theta})\left[\cos e+\sin e \tan \bar{\theta} \frac{E_{2}(e)-\sin ^{2}(\psi / 2) E_{2}(i)}{2-E_{1}(e)-(\psi / \pi) E_{1}(i)}\right] \\
S(i, e, \psi) & \simeq \frac{\mu_{e}}{\mu_{e}(0)} \frac{\mu_{0}}{\mu_{0 e}(0)} \frac{\chi(\bar{\theta})}{1-f(\psi)+f(\psi) \chi(\bar{\theta})\left[\mu_{0} / \mu_{0 e}(0)\right.}
\end{aligned}
$$

If $i \geq e$,

$$
\begin{aligned}
\mu_{0 e} & \simeq \chi(\bar{\theta})\left[\cos i+\sin i \tan \bar{\theta} \frac{E_{2}(i)-\sin ^{2}(\psi / 2) E_{2}(e)}{2-E_{1}(i)-(\psi / \pi) E_{1}(e)}\right] \\
\mu_{e} & \simeq \chi(\bar{\theta})\left[\cos e+\sin e \tan \bar{\theta} \frac{\cos \psi E_{2}(i)+\sin ^{2}(\psi / 2) E_{2}(e)}{2-E_{1}(i)-(\psi / \pi) E_{1}}\right] \\
S(i, e, \psi) & \simeq \frac{\mu_{e}}{\mu_{e}(0)} \frac{\mu_{0}}{\mu_{0 e}(0)} \frac{\chi(\bar{\theta})}{1-f(\psi)+f(\psi) \chi(\bar{\theta})\left[\mu / \mu_{e}(0)\right]}
\end{aligned}
$$

where

$$
\begin{aligned}
\chi(\bar{\theta}) & =\frac{1}{\left(1+\pi \tan ^{2} \bar{\theta}\right)^{1 / 2}} \\
E_{1}(x) & =\exp \left(-\frac{2}{\pi} \cot \bar{\theta} \cot x\right) \\
E_{2}(x) & =\exp \left(-\frac{1}{\pi} \cot ^{2} \bar{\theta} \cot ^{2} x\right) \\
f(\psi) & =\exp \left(-2 \tan \frac{\psi}{2}\right)
\end{aligned}
$$

Therefore, the bidirectional reflectance from the rough surface is modeled by,

$$
r(i, e, \alpha)=\frac{\omega}{4 \pi} \frac{\mu_{0 e}}{\mu_{0 e}+\mu_{e}}\left\{[1+B(\alpha)] P(\alpha)+H\left(\mu_{e}\right) H\left(\mu_{0 e}\right)-1\right\} S(i, e, \alpha)
$$

The roughness parameter counts as a geometric parameter which should be independent of wavelength. The interpretation of the photometric roughness, $\bar{\theta}$ is scrutinized by Helfenstein (1988) to compare the value of $\bar{\theta}$ with the one measured geologically. Results 
from this study confirm that the roughness derived from Hapke's photometric model is an integral of roughness properties over all scales extending to the upper limit resolution of available photometric data. Further investigations by Shepard and Campbell (1998) indicate that photometric roughness can be affected by the shadows in the small topographic scales. The contribution of shadows causes to undervalue the actual roughness of the surface. The $\bar{\theta}$ value derived from the disk-resolved reflectance analysis of lunar data, show reasonably well that the lunar highland regolith is rougher than lunar mare areas (Helfenstein and Shepard, 1999).

\subsubsection{Empirical Surface Scattering Models}

In literature, the bidirectional reflectance of a regolith is described by several simple expressions, considering their intrinsic limitations. These expressions provide an adequate explanation for the scattering behavior of surfaces.

\subsubsection{Lambert Model}

Lambert reflectance model describes the scattered light in a basic way. It assumes that the reflectance changes simply with the cosine of incidence angle, cos $i$,

$$
r_{L}(i, e, \alpha)=\frac{1}{\pi} A_{L} \mu_{0}
$$

The model includes beside the $\mu_{0}$, the Lambert albedo $A_{L}$. When scattering properties of a surface can be explained by Lambert model, the surface is addressed as a diffuse surface or Lambert surface. To be precise, $A_{L}$ presented the directional-hemispherical reflectance of a Lambert surface. The surface can be defined as a perfectly diffuse surface in case of $A_{L}=1$. The Lambert model describes sufficiently the reflected light behavior from high-albedo surfaces such as snow with a simple mathematical expression, but shows less good results for low albedo surfaces like for asteroids and comet nuclei (Hapke and Wells, 1981; Pinty et al., 1989).

\subsubsection{Minnaert Model}

Minnaert (1941) provided a generalized Lambert model which results in a photometric function of the form

$$
r_{M}(i, e, \alpha)=A_{M}\left(\mu_{0}\right)^{k} \mu^{k-1}
$$

where both constants of $A_{M}$ and $k$ are determined empirically. $A_{M}$ is called Minnaert albedo, and $k$ is known as the limb darkening coefficient. On the condition that $k=1$, Minnaert model shrinks to the Lambert model, and $A_{M}=A_{L} / \pi$.

The Minnaert model is able to describe the bidirectional reflectance of numerous surfaces usually for small phase angles. The Minnaert parameters are functions of wavelength and phase angle. $A_{M}$ versus phase angle actually is comparable with the surface phase curve of the object, and versus wavelength it resembles the reflectance spectrum of the surface. However, $k(\alpha, \lambda)$ cannot be easily interpreted according to physical properties of the surface; $k$ presents the degree of limb darkening, e.g., $k=0.5$ indicates that there is no limb darkening, while limb darkened surfaces have $k>0.5$. 


\subsubsection{Lommel-Seeliger Model}

Diffuse reflection can be modeled physically in a simple way by the Lommel-Seeliger law. The law is only included isotropic scatterers, and is acceptable for low-albedo particulate media where single scattering is dominant (Hapke, 1963a). The expression for this model is given by,

$$
r_{L S}=\frac{\omega}{4 \pi} \frac{\mu_{0}}{\mu_{0}+\mu}
$$

Based on the study by KenKnight et al. (1967), the Lommel-Seeliger Model describes the relative brightness across the surface of the moon quite well. For this reason, the Lommel-Seeliger photometric function is also known as Lunar-like photometric function.

\subsubsection{Lunar-Lambert Model}

The photometric models of the Lommel-Seeliger (Lunar-like function) and of the Lambert are merged to construct another empirical photometric function, the Lunar-Lambert function, for instance used by McEwen (1991) for the first time in the form of,

$$
r_{L L}=A_{L L}\left[(1-L) \mu_{0}+2 L \frac{\mu_{0}}{\mu_{0}+\mu}\right]
$$

In Equation 2.52, $L$ is representing the level of limb darkening, and $A_{L L}$ is known as LunarLambert albedo. $L$ is a function of phase angle similar to the Minnaert limb darkening parameter $k$, but the variation trend is different.

\subsection{Quantities related to disk-integrated reflectance}

The fundamental parameters of geometric albedo, the phase integral, and the Bond albedo which are necessary for studying the disk-averaged brightness with respect to phase angle, along with, the Hapke equation for the treatment of disk-integrated reflectance are summarized in this section (Hapke, 1993).

\subsubsection{The geometric albedo}

The geometric albedo (known as the physical albedo) $A_{p}$, is the ratio of reflectance from the body of radius $\mathrm{R}$ at zero phase angle to the reflectance from a perfect Lambert disk of the same radius and at the same distance as the body, but illuminated and viewed perpendicularly. Thus, if the power per unit area in the collimated light is the irradiance $\mathrm{J}$, then the total scattered power from an area element of $\mathrm{dA}$, toward the direction of the detector is $\operatorname{Jr}(i, e, \alpha) \mu d A$. Hence, the geometric albedo for a spherical body of radius $\mathrm{R}$ over all the illuminated area is given by,

$$
A_{p}=\frac{\int_{A(i)} J r(e, e, 0) \mu d A}{(J / \pi) \pi R^{2}}=R^{-2} \int_{A(i)} r(e, e, 0) \mu d A
$$

where $d A=2 \pi R^{2}$ sin ed $e=2 \pi R^{2} d \mu$ represents the surface elements of the body, and A(i) is the area of the illuminated hemisphere. Replacing $r(e, e, 0)$ with Equation 2.30, and 
approximating the $\mathrm{H}$-function within the integral, the geometric albedo in terms of Hapke parameters is given by,

$$
A_{p} \simeq r_{0}\left(\frac{1}{2}+\frac{1}{6} r_{0}\right)+\frac{\omega}{8}\left[\left(1+B_{0}\right) p(0)-1\right]
$$

where $r_{0}=1-\gamma / 1+\gamma$. A quantity, $\gamma$, is defined as the albedo factor of medium and is equal to $\sqrt{1-\omega}$.

\subsubsection{The phase integral}

The phase integral which is an expression to characterize a directional scattering behavior of a surface, is defined as

$$
q=2 \int_{0}^{\pi} \frac{I(\alpha)}{I\left(0^{\circ}\right)} \sin \alpha d \alpha
$$

where the fraction $\frac{I(\alpha)}{I\left(0^{\circ}\right)}$ considers the disk-integrated brightness normalized to zero phase angle.

\subsubsection{The Bond albedo}

The Bond albedo (also called the spherical albedo) is a parameter for the total fraction of incident irradiance reflected from a body into all direction. It is used to understand the energy balance of the body. The Bond albedo, $A_{s}$, is related to the geometrical albedo, $A_{p}$, and the phase integral, q, and is given by,

$$
A_{s}=A_{p} \times q
$$

The value of Bond albedo cannot exceed unity due to conversation of energy.

\subsubsection{The Hapke disk-integrated model}

The disk-integrated phase function of an object can be only modeled by assuming the regular shapes such as spheres or ellipsoids. (Hapke, 1984, 1986) derived the disk-integrated bidirectional reflectance equation of rough surfaces for spherical bodies. This equation is given by

$$
\begin{aligned}
\frac{I}{F}(\alpha) & =\left[\left\{\frac{\omega}{8}[(1+B(\alpha)) p(\alpha)-1)\right]+r_{0}\left(1-r_{0}\right)\right\} \\
& \times\left\{1-\sin \left(\frac{\alpha}{2}\right) \tan \left(\frac{\alpha}{2}\right) \ln \left[\cot \left(\frac{\alpha}{4}\right)\right]\right\} \\
& \left.+\frac{2}{3} r_{0}\left(\frac{\sin \alpha+(\pi-\alpha) \cos \alpha}{\pi}\right)\right] \mathcal{S}(\alpha, \bar{\theta})
\end{aligned}
$$

where $\mathcal{S}(\alpha, \bar{\theta})$ is a correction factor for the surface macroscopic roughness. For $\alpha \leq 60^{\circ}$, it can be analytically approximated by

$$
\mathcal{S}(\alpha, \bar{\theta}) \approx \exp \left\{\left[-0.32 \sqrt{\tan \bar{\theta} \tan \frac{\alpha}{2}}-0.52 \tan \bar{\theta} \tan \frac{\alpha}{2}\right] \bar{\theta}\right\}
$$


where the unit of $\bar{\theta}$ is in radian. When $\alpha>60^{\circ}$, Equation 2.58 overestimate the value of the roughness correction factor, $S(\alpha, \bar{\theta})$. Hence one must use the tabulated data, which are available for a certain value of $\bar{\theta}$ (Hapke, 1993). The tabulated data can be presented by two-dimensional polynomial equation. In Appendix A, we attempted to employ twodimensional polynomial fitting to obtain an overall function for $\mathcal{S}(\alpha, \bar{\theta})$. However, it is common to use Equation 2.58 for the entire range of phase angles in the Hapke diskintegrated modeling as we did for our analyses in this dissertation. 



\section{ROSETTA-OSIRIS Images}

\subsection{ROSETTA, the ESA's mission}

ROSETTA is a mission for planetary science of the European Space Agency (ESA). The mission is a long-term program a cornerstone with 10 years journey to scrutinize its key objective, the Jupiter-family comet $67 \mathrm{P} /$ Churyumov-Gerasimenko. The mission name is taken from a plate of volcanic basalt called the Rosetta Stone. The Rosetta plate was the key to decipher the hieroglyph of the ancient Egypt civilization. As a matter of fact, the name Rosetta is selected for the spacecraft due to similarity of its goal. The Rosetta probe is developed to figure out the physical and compositional properties of a comet, the least processed material in our solar system from the formation era of the Sun and its planets (Schulz et al., 2009).

The Rosetta spacecraft was launched on 2nd of March 2004 from Kourou/French Guiana with an Aiane-5 rocket. On its way to the comet, Rosetta performed close fly-bys at two interesting objects in the main asteroid belt, asteroid (2867) Steins and asteroid (21) Lutetia. The asteroid fly-by scenario as well as related mission operation are summarized in Sections 3.3.1 and 3.3.2.

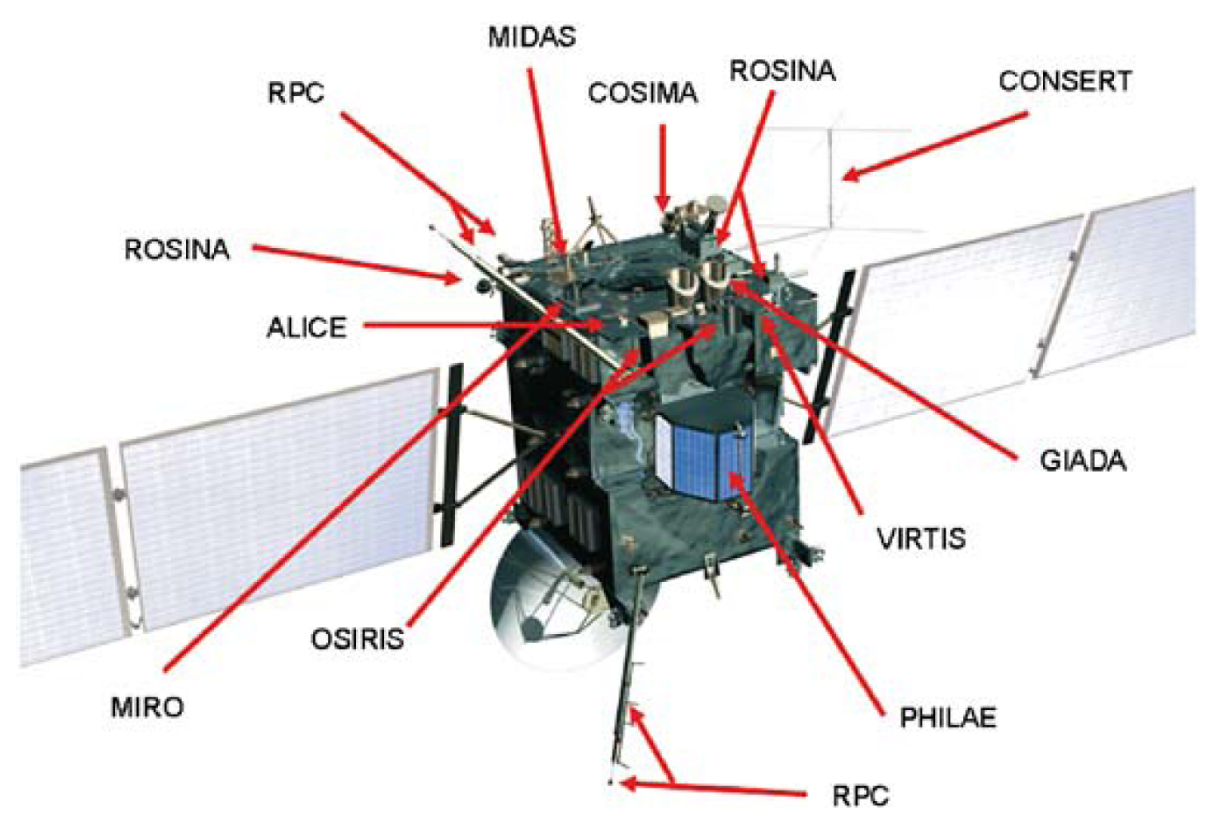

Figure 3.1

The main science goals of the Rosetta mission are focused on the determination of the 
global and local characterization of the nucleus, investigating the cometary activity, and the interaction of comet with the solar wind during its perihelion. The Rosetta spacecraft comprises two parts, the orbiter with 11 scientific instruments (see Table 3.1), and the lander PHILAE with 10 scientific experiments for the surface of nucleus(see Table 3.2). The orbiter instruments attempt to monitor the physical and chemical properties of the nucleus and in the vicinity of the nucleus. The PHILAE instruments examine directly the nucleus material. The Rosetta is now orbiting the comet at $r=3.6$ AU. Between June to October 2014 at solar distances 4.5 -3.1 AU, Rosetta approached the comet from about a few million to a few $10 \mathrm{~km}$ entering into the gaseous and dusty environment of the coma and analyzing the nucleus.

On 11 November 2014 the Philae down to the surface and landed successfully. Afterward, the orbiter and the lander will commence the individual and continued scientific programs, and continue their tasks all the way of the comet to the perihelion and beyond. Figure 3.1 displays an illustration of the Rosetta spacecraft and its scientific equipments. 
Table 3.1: The 11 scientific equipment on board of the orbiter of Rosetta mission

Instrument Name Scientific Goals

ALICE UV imaging spectrograph, analyzing gases in the coma and tail of the comet, detecting water, carbon monoxide, and carbon dioxide.

CONSERT COmet Nucleus Sounding Experiment by Radiowave Transmission, examining the nucleus interior by analyzing reflected radio wave transmitted between the spacecraft and the lander

COSIMA Secondary Ion Mass Analyzer, investigating the chemical compositions of the cometary dust.

GIADA Grain Impact Analyzer and Dust Accumulator, measuring the number, mass, momentum and velocity distribution of dust grains.

MIDAS Micro Imaging Dust Analysis System, examining the dust surrounding the comet and delivering details of particle population, size, shape and volume.

MIRO Microwave Instrument of the Roestta Orbiter, verifying the abundance of molecules in the cometary nucleus and essential isotope ratios, determining the nucleus surface and subsurface temperatures and measuring the surface outgassing rate.

OSIRIS Optical, Spectroscopic and Infrared Remote Imaging System, Multi-Color camera system with a Narrow and a Wide Angle Camera to take high resolution and wide-angle images of the nucleus.

ROSINA Rosetta Orbiter Spectrometer for Ion and Neutral species Analysis, specifying the composition, temperature and the bulk velocity of the gas in the comet's atmosphere and ionosphere

RPC

Rosetta Plasma Consortium, monitoring entirely the plasma environment around the comet.

RSI Radio Science Investigation, using the frequency shifts of the spacecraft's radio signals to calculate the mass and gravity.

VIRTIS Visible and Infrared Thermal Imaging Spectrometer, recording in details the temperature of the nucleus's surface, monitoring the comet gases, constraining the physical characteristics of the coma 
Table 3.2: The 10 scientific instruments of the Rosetta Lander PHILAE

Instrument Name Scientific Goals

APXS

Alpha Particle, X-ray spectrometer, measuring the elemental composition of the surface material.

CIVA

Comet nucleus Infrared and Visible Analyzer panoramic and microscopic imaging system to characterize the samples and record the IR spectra of samples.

CONSERT The lander PHILAE equipped with the CONSERT instrument which has the same task as the one on the Rosetta orbiter

COSAC COmetary SAmpling and Composition experiment, a gas analyzer to detect organic molecules in the comet materials.

MUPUS MUlti PUrpose Sensor for surface and subsurface science, measuring the thermal and mechnical properties of the surface layers

ROLIS ROsetta Lander Imaging System, a downward looking camera for exploring and photographing the landing site before and after Philae has landed.

ROMAP ROsetta lander MAgnetometer and Plasma monitor, exploring the magnetic field and plasma of the landing site, evaluating the interaction between the landing site environment and the solar wind

SESAME $\quad$ electrical, acoustic and dust impact monitoring at the surface.

SD-2 Drill, Sampler and Distribution (SD2) subsystem, extracting the soil of comet nucleus at discrete depths

PTOLEMY gas chromaograph and isotope ratio mass spectrometer, studying the comet surface and subsurface from mass spectroscopy point of view. 


\subsection{OSIRIS, the camera system}

The Optical, Spectroscopic, and Infrared Remote Imaging system, OSIRIS is the scientific imaging system onboard the Rosetta space probe. OSIRIS consists of two units, a high resolution Narrow Angle Camera (NAC) and a Wide Angle Camera (WAC) (for details (Keller et al., 2007)). Here, I summarizes the instrument description in brief. The OSIRIS instrument is designed to obtain images of comet $67 \mathrm{P} /$ churyumov-Gerasimenko through WAC and NAC cameras. The scientific objectives and thus the design of the camera system focus on the exploration of nucleus surface as well as the dust ejection and gas emission processes near the nucleus.

The NAC unit has an angular resolution of $18.6 \mu \mathrm{rad} \mathrm{px}^{-1}$ and contains a set of 12 filters in the wavelength range of $250-1000 \mathrm{~nm}$. The NAC is devised to take high resolution images of its target with a field of view (FOV) of $2.2^{\circ} \times 2.2^{\circ}$. The NAC filters are employed to characterize the reflectivity spectrum of the surface and to find possible absorption bands. For instance, there is a group of filters in the wavelength range of 800 $\mathrm{nm}$ to $1 \mu \mathrm{m}$ to search for possible pyroxene and olivine absorptions. The band-passes of NAC filters are relatively wide, normally 24-100 nm (Figure 3.2, Table 3.4).

The WAC images are taken at an angular resolution of $101 \mu \mathrm{rad} \mathrm{px}^{-1}$. The resolving power of the WAC is five times less than the NAC, but it has a wider field of view. The field of view is $11.35^{\circ} \times 12.11^{\circ}$. The WAC unit includes a set of 14 filters in the wavelength range of 240-720 nm (Figure 3.3, Table 3.5). The majority of WAC filters are narrow band filters fitted to gas emission study. Two broad-band filters were included for the WAC, the IR WAC filter for detecting the nucleus, in the case of NAC failure, and the green WAC filter, exactly the same as the NAC filter, for cross-checking. The WAC filters are mainly designed to investigate gas and radical emission. Specifically, $\mathrm{NH}$ at $336.2 \mathrm{~nm}$ and $\mathrm{NH}_{2}$ at $570 \mathrm{~nm}$ are measured to study $\mathrm{NH}$ and $\mathrm{NH}_{2}$ gas mission. To find evidence of $\mathrm{H}_{2} \mathrm{O}$, $\mathrm{OH}-\mathrm{WAC}$ filter at $309 \mathrm{~nm}$ and OI-WAC filter at $630 \mathrm{~nm}$ are selected. Other gas emissions such as $\mathrm{CN}$ and sodium are detectable by CN-WAC filter at $388.4 \mathrm{~nm}$ and Na-WAC filter at $590.7 \mathrm{~nm}$. The basic parameters of OSIRIS camera system are tabulated in Table 3.3 .

Table 3.3: Specifications of the NAC and WAC systems

\begin{tabular}{lcc}
\hline Basic parameters & NAC & WAC \\
\hline Optical design & 3-mirror off-axis & 2-mirror off-axis \\
Angular resolution [ $\mathrm{rrad} \mathrm{px}^{-1}$ ] & 18.6 & 101 \\
Focal length [mm] & 717.4 & \\
Mass [kg] & 13.2 & 9.48 \\
Field of View [deg] & $2.20 \times 2.22$ & $11.35 \times 12.11$ \\
Typical filter bandpass [nm] & 40 & 5 \\
Wavelength range [nm] & $250-1000$ & $240-720$ \\
Number of filters & 12 & 14 \\
\hline
\end{tabular}

The raw images (in units of digital numbers, DN) are processed through the standard OSIRIS calibration pipeline which considers all typical corrections for CCD cameras. The images were then radiometrically calibrated (i.e., converted to units of $\mathrm{W} \mathrm{m}^{-2} \mathrm{~nm}^{-1}$ $\mathrm{sr}^{-1}$ ) and corrected for geometrical distortion. 
Table 3.4: The Narrow Angle Camera Filters

\begin{tabular}{llcc}
\hline Filter ID & Filter Name & $\lambda_{c}(\mathrm{~nm})$ & number of images \\
\hline F15 & FFP-UV Far-UV & 269.3 & 12 \\
F16 & FFP-UV Near-UV & 360.0 & 14 \\
F84 \& F24 & Neutral Blue & 480.7 & 55 \\
F83 \& F23 & Neutral Green & 535.7 & 15 \\
F82 \& F22 & Neutral Orange & 649.2 & 172 \\
F87 \& F27 & Neutral Hydra & 701.2 & 12 \\
F88 \& F28 & Neutral Red & 743.7 & 16 \\
F58 & Ortho Red & 790.5 & 7 \\
F51 & Ortho FFP-IR & 805.3 & 11 \\
F41 & Near-IR FFP-IR & 882.1 & 14 \\
F61 & $\mathrm{Fe}_{2} \mathrm{O}_{3}$ FFP-IR & 931.9 & 12 \\
F71 & IR FFP-IR & 989.3 & 14 \\
\hline
\end{tabular}

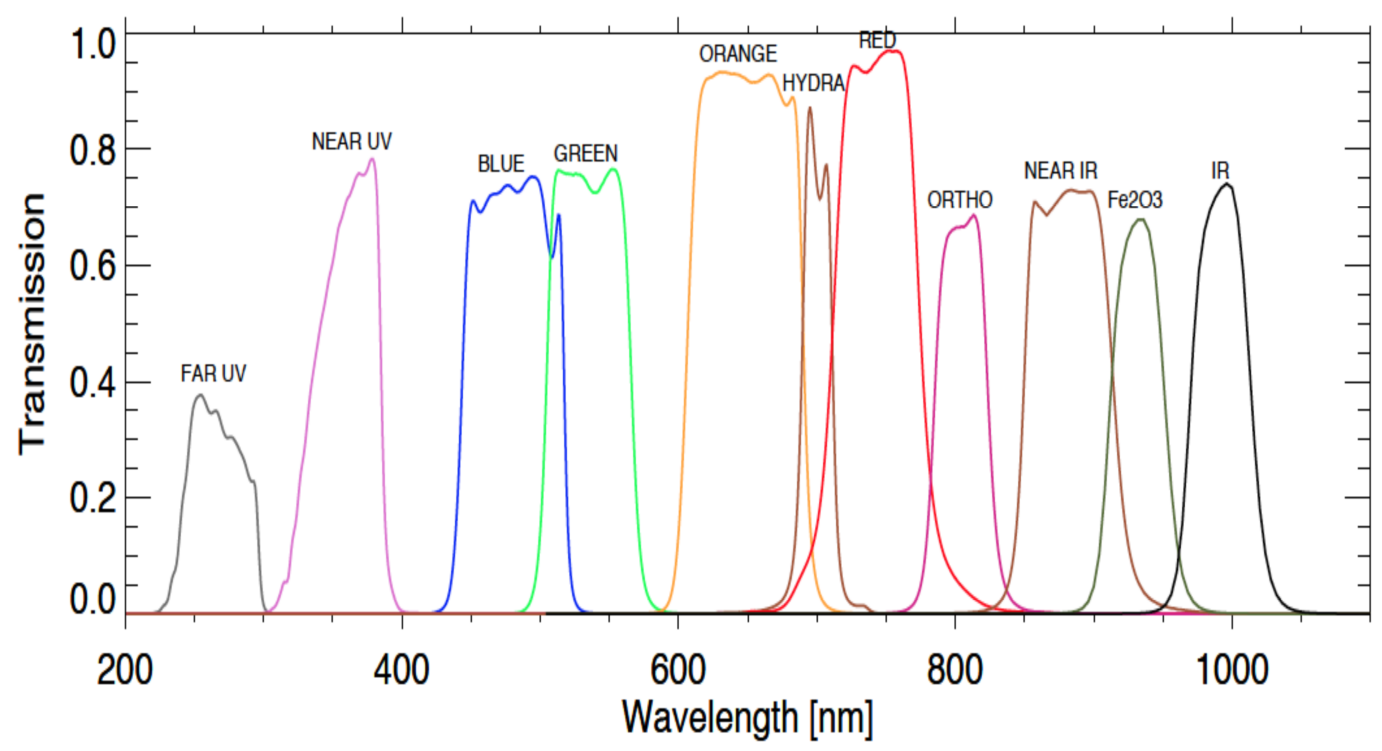

Figure 3.2: The Transmission curves of the 11 OSIRIS NAC filters. (Holger Sierks, priv. comm.) 
Table 3.5: The Wide Angle Camera Filters

\begin{tabular}{llcc}
\hline Filter ID & Filter Name & $\lambda_{c}(\mathrm{~nm})$ & Number of images \\
\hline F31 & UV-245 & 246.2 & 13 \\
F51 & UV-295 & 295.9 & 19 \\
F61 & OH & 309.7 & 9 \\
F71 & UV-325 & 325.8 & 15 \\
F81 & NH & 335.9 & 3 \\
F13 & UV-375 & 375.6 & 45 \\
F21 & Green & 537.2 & 4 \\
F15 & $\mathrm{NH}_{2}$ & 572.1 & 15 \\
F16 & Na & 590.7 & 21 \\
F18 & Vis-610 & 612.6 & 21 \\
F12 & Red & 629.8 & 40 \\
F17 & OI & 631.6 & 100 \\
\hline
\end{tabular}

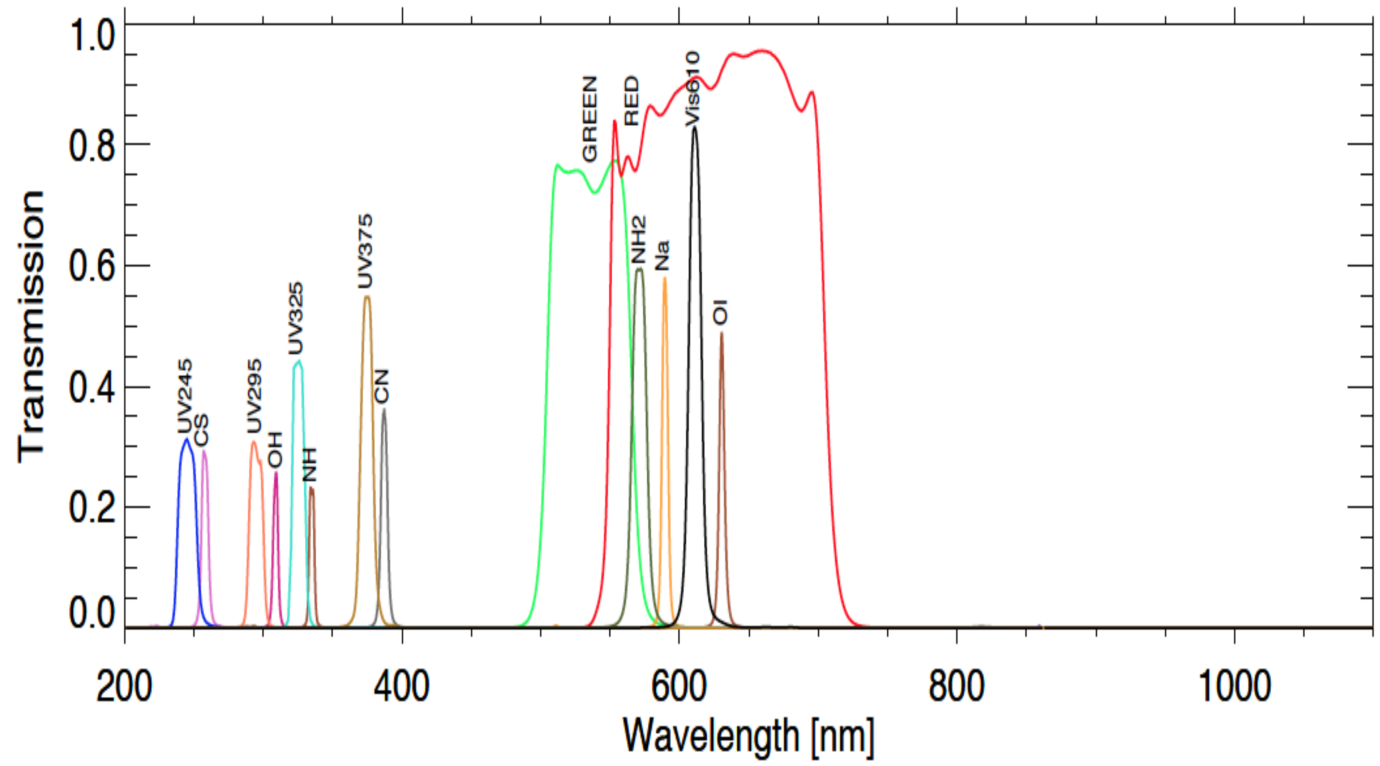

Figure 3.3: The Transmission curves of the 14 OSIRIS WAC filters.(Holger Sierks, priv. comm.) 


\subsection{Rosetta asteroid fly-bys}

\subsubsection{Rosetta fly-by at Asteroid (2867) Steins}

Rosetta approached asteroid Steins with the relative velocity of $8.6 \mathrm{~km} / \mathrm{s}$ on 5 th September 2008 at a heliocentric distance of 2.14 AU. The spacecraft reached the closest possible distance to the asteroid (CA) of $803 \mathrm{~km}$ at 18:38:20 UTC. The phase angle of asteroid Steins was equal to $38^{\circ}$ when Rosetta met the object and a minimum of $0.36^{\circ} 2 \mathrm{~min}$ before CA. At a later time, the sun-asteroid-spacecraft angle increased to $51^{\circ}$, where the CA took place and then it ended up of $141^{\circ}$ (Figure 3.4). For more details, the reader refers to Accomazzo et al. (2010) and Schulz (2010).

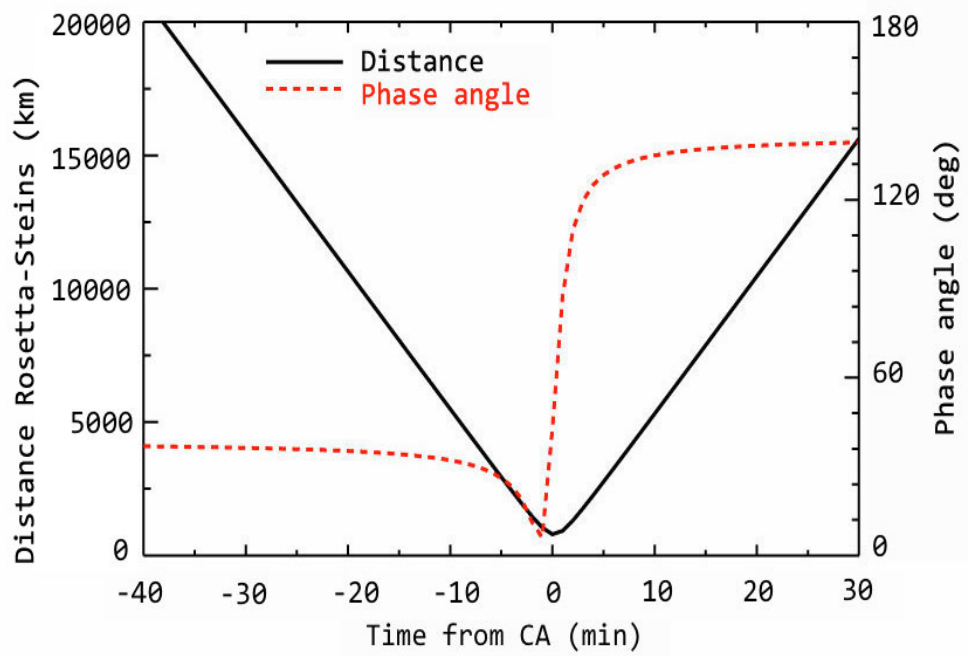

Figure 3.4: Distance-phase angle versus Rosetta flyby time for asteroid steins. The closest approach time is zero. The data used for plot are taken from http://ssd.jpl.nasa. gov/?horizons (OSIRIS operation team, priv. comm).

The 14 scientific instruments of Rosetta were activated during its fly-by at asteroid Steins (Accomazzo et al., 2010). The scientific camera system OSIRIS monitored the asteroid through only one of its camera, the WAC, because the NAC stopped working 10 min before CA. In the time of the Steins flyby, the WAC images recorded by OSIRIS, spanning a wavelength range of 295 to $630 \mathrm{~nm}$ (Keller et al., 2010).

\subsubsection{Rosetta fly-by at Asteroid (21) Lutetia}

Rosetta visited the second asteroid target of mission in the main belt, asteroid(21) Lutetia on 10 July 2010. The flyby at Lutetia occurred at a heliocentric distance of $2.72 \mathrm{AU}$. Rosetta flew by the asteroid with a velocity of $15 \mathrm{~km} / \mathrm{s}$. The CA was reached on 15:45 UT at a distance of $3168.2 \mathrm{~km}$. The spacecraft arrived at the asteroid when the object was at low phase angle around $10^{\circ}$ (Figure 3.5). The asteroid moved to zero phase angle $18 \mathrm{~min}$ 
before CA; at the time that the interval between the object and spacecraft was $16400 \mathrm{~km}$ (Schulz et al., 2012).

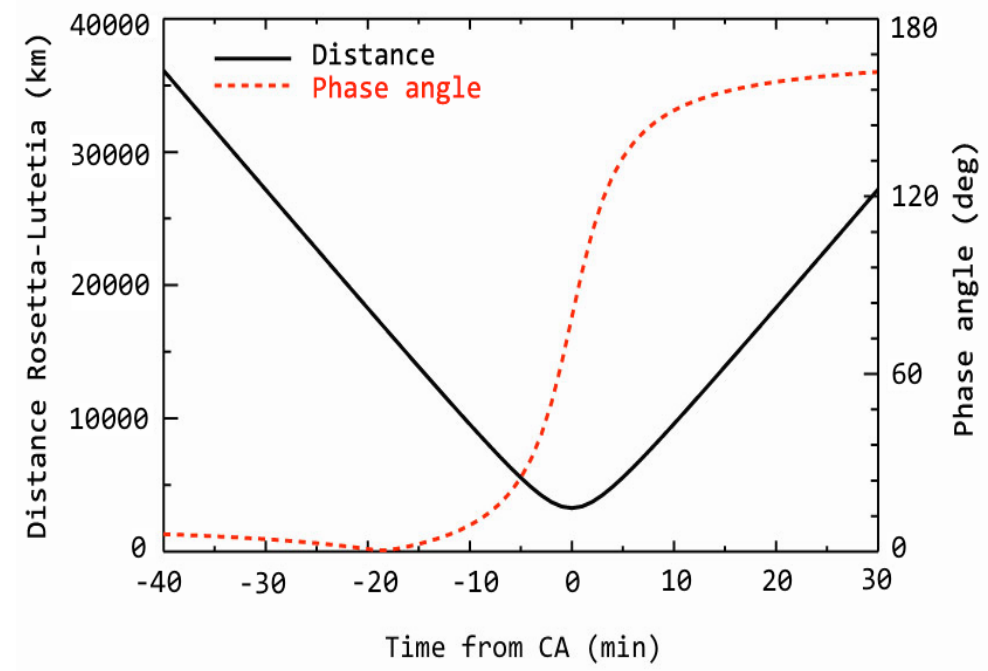

Figure 3.5: Distance-phase angle against Rosetta flyby time for asteroid Lutetia. The closest approach time is zero. The data used for plot are taken from http://ssd.jpl. nasa.gov/?horizons (OSIRIS operation team, priv. comm).

Nine of the eleven experiments on board Rosetta were switched on to examine asteroid Lutetia. Besides, three instruments of the lander Philae were working to monitor the environment near the asteroid (Schulz et al., 2012). The OSIRIS (Keller et al., 2007) Sierks et al. 2011) cameras delivered the images for almost 10h during the flyby, from 9 hours and $30 \mathrm{~min}$ before closest encounter until $16 \mathrm{~min}$ after. The total 462 images, 234 by the NAC and 228 by the WAC were taken during the flyby. The northern hemisphere of the asteroid was mostly covered by these images, more than half of the asteroid surface. The images near CA were acquired around phase angles $\left(60^{\circ}-70^{\circ}\right)$ to recognize the surface feature of the target (Sierks et al., 2011). 



\section{Surface reflectance analysis of asteroid (2867) Steins}

\subsection{Overview of Steins' surface}

The surface of asteroid Steins is resolved by OSIRIS images during the Rosetta flyby on September of 2008 (Keller et al., 2010). These images, taken at different phase angles and with different color filters, provided understanding of geomorphological and photometric variations across the surface of Steins (Marchi et al., 2010; Besse et al., 2012; Schröder et al., 2010; Leyrat et al., 2010).

The largest crater with a diameter of $2.1 \mathrm{~km}$ is found near the south pole of the object. Chains of deep recesses (crater-like) from the south to the north pole are also observed on Steins (see Figure 4.1 panel a). Another noticeable feature is a large depression which is discovered in NAC images, covering a distinct side of Steins' surface (see Figure 4.1 panel b). 42 craters have identified on the surface of Steins. 31 craters are detectable in WAC image (the western side), while much less craters, 12, were found in the NAC image (the eastern side and front), with one shared. This remarkable difference between two surface parts of Steins imaged by the NAC and WAC cameras, however it can be related to disapproving illumination geometry and the low resolution, such distinction with the a ratio of 3.6 for all range sizes of craters is not explainable (Besse et al., 2012).

The depth-to-diameter (d/D) ratio of the craters on the surface of Steins is studied by Besse et al. (2012), suggesting the distribution of the $\mathrm{d} / \mathrm{D}$ ratio can be divided into three categories: shallow $(\mathrm{d} / \mathrm{D}<0.11)$, intermediate $(0.11<\mathrm{d} / \mathrm{D}<0.15)$, deep craters $(\mathrm{d} / \mathrm{D}>0.15)$ (Figure 4.2). The shallow and intermediate craters have an almost uniform distribution over the surface of Steins and represent almost the same amount of the total population of craters, $38 \%$ and $45 \%$, respectively. The deep craters which are mostly located near the large crater on the south pole of Steins, constituted $17 \%$ of the whole population.

The surface variegation of asteroid Steins is studied by Schröder et al. (2010) and Leyrat et al. (2010), using OSIRIS images. No significant color variegation is reported by Leyrat et al. (2010), using color ratios of the 630.6-nm/571.1-nm and 571.1-nm/386.9-nm filters. However, Schröder et al. (2010) claimed a color variation for the inner part of the large crater on the south pole. Color ratio images of 630-nm/296-nm ratio and 630$\mathrm{nm} / 326-\mathrm{nm}$ indicate that the interior surface of the large crater is $5-10 \%$ is bluer than average.

The Hapke disk-resolved modeling was performed using the OSIRIS WAC images of Steins (Spjuth et al., 2012), applying a new technique for the extraction of the bidirectional reflectance. The Hapke parameters of Steins was also modeled from its disk- 


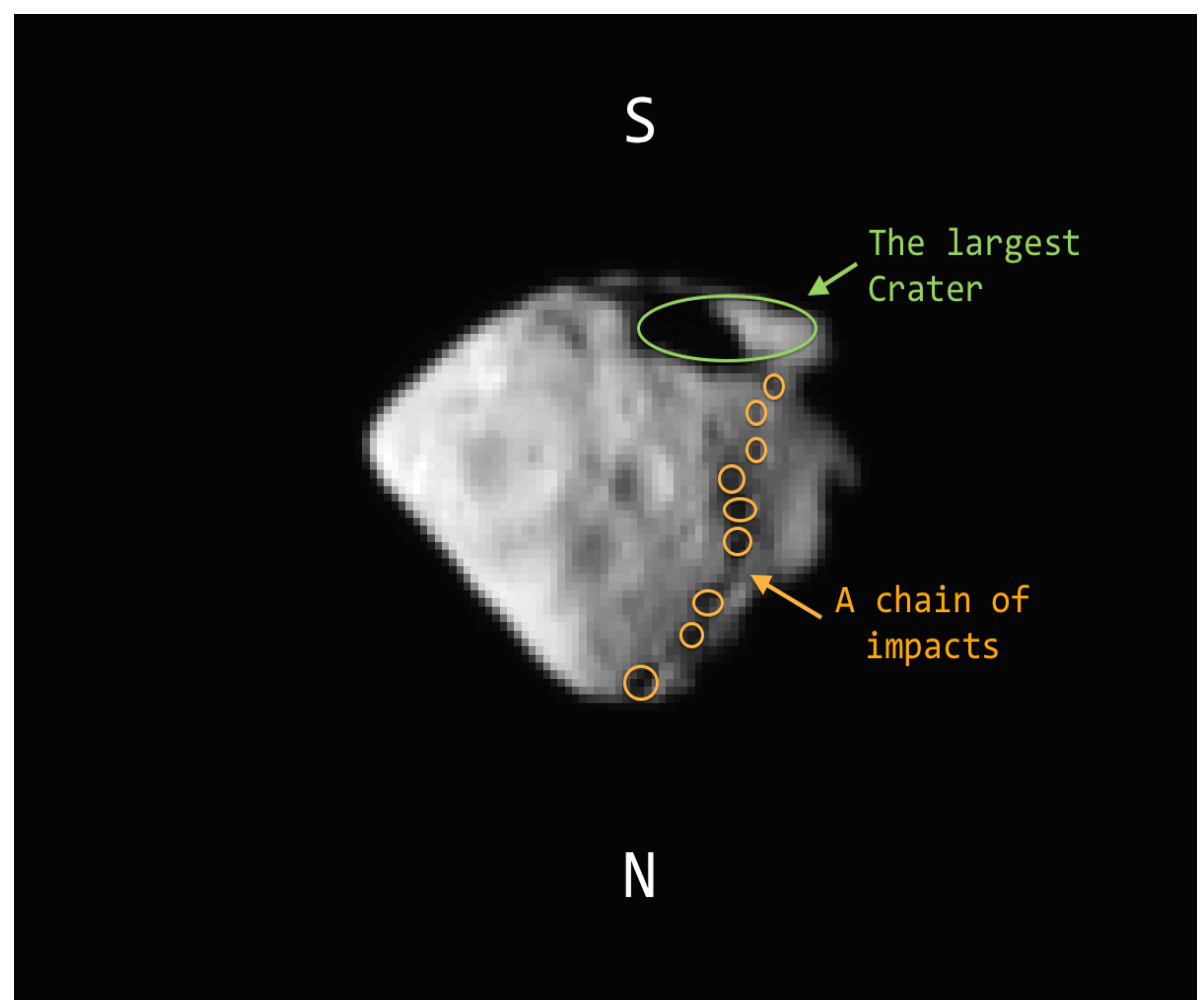

(a)

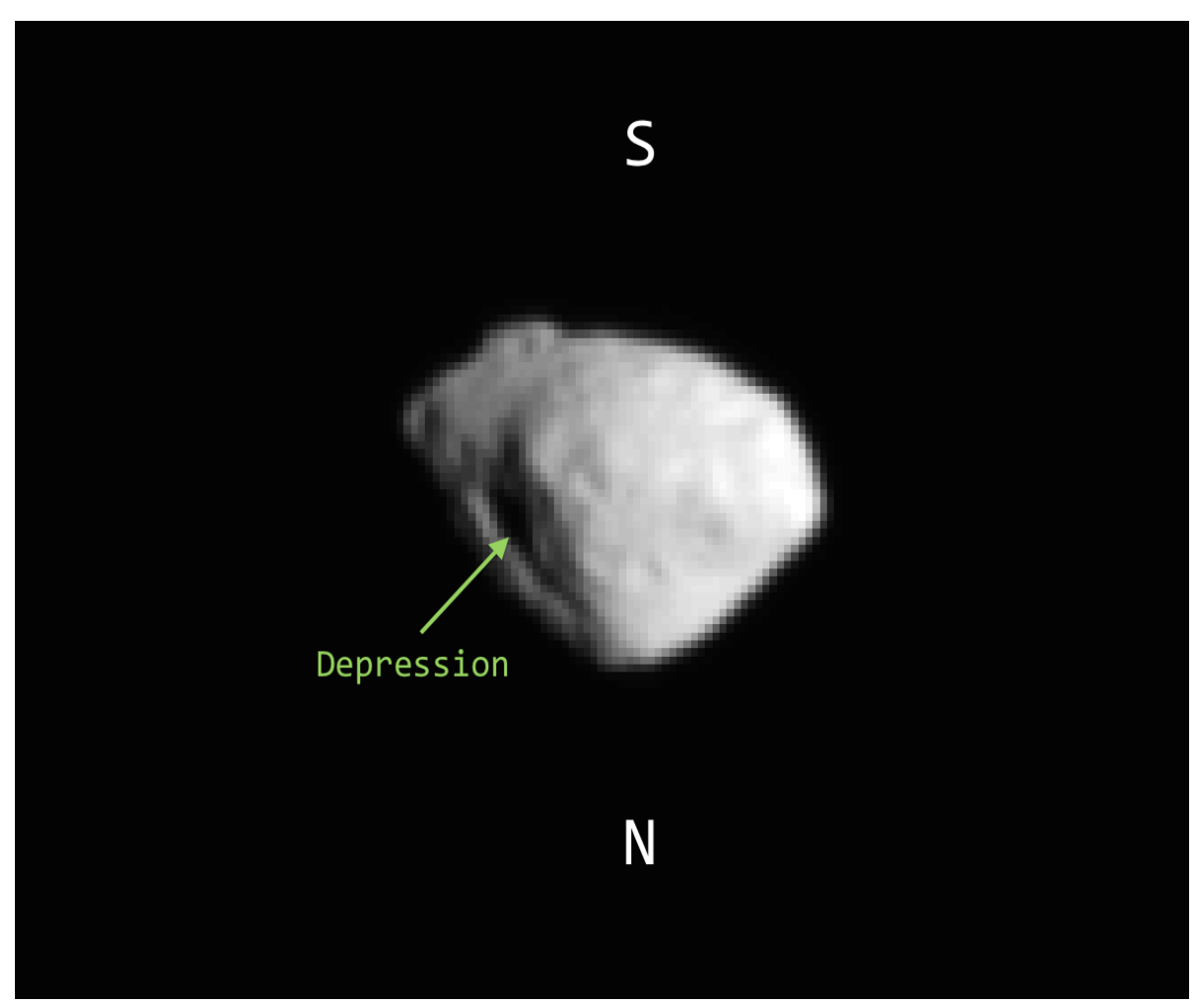

(b)

Figure 4.1: Panel (a) displays a WAC F17 image taken at the closest approach (CA) at $\lambda=631.6 \mathrm{~nm}$. The largest crater and the pit-chain are labeled in green and orange, respectively. Panel (b) displays a NAC image acquired before CA. The large depression is marked with a green arrow. 


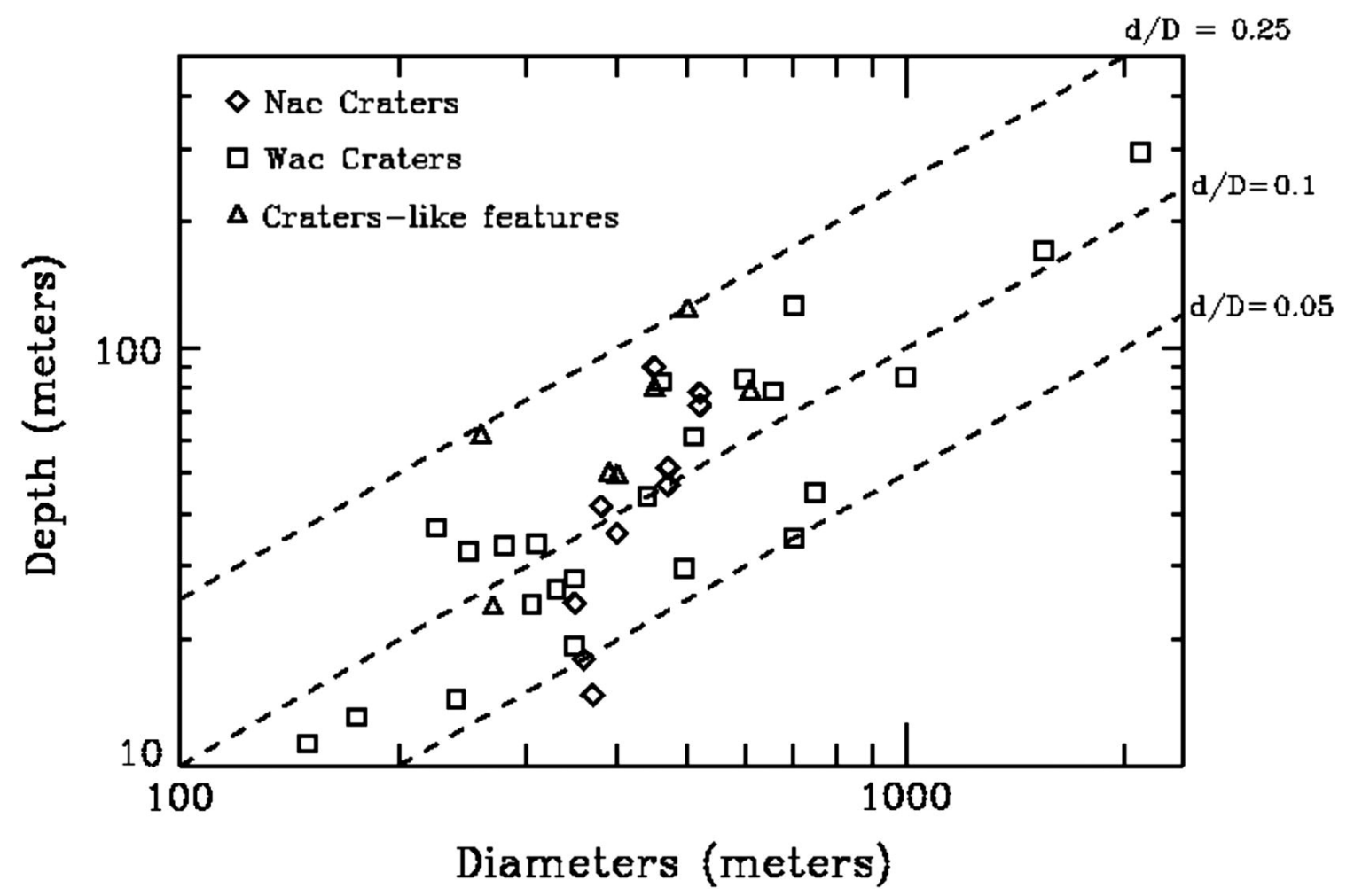

Figure 4.2: The scatter plot of depth-to-diameter ratio of all craters identified on the WAC and NAC images of Steins, taken from Besse et al. (2012)

integrated phase function by Forgia et al. (2012) without applying the available shape model of Steins.

As mentioned above, the photometric parameters of Steins are already obtained, using disk-averaged and disk-resolved data. In this chapter, the aim is to validate our technique, comparing our results for asteroid with those previously analyzed.

\subsection{Data extraction}

In this section, we describe the way we chose to extract intensity and geometric angles from the observed OSIRIS WAC images of asteroid Steins. For this purpose, the shape model of asteroid is used. The high-resolution shape model of Steins (Jorda et al., 2012) has 20480 triangular facets. For analysis of the WAC images, the shape model is needed to be degraded to be suitable with the resolution of images. We degraded $10 \%$ the number of facets in the original shape model. The degraded shape model of Steins with 2048 triangular facets is displayed in Figure 4.3. The size of facets varies in the degraded shape model between 3 to 8 image pixels.

We have to align the shape model and the observed images for the extraction of brightness at facet level. We implemented the co-alignment manually by shifting and/or rotating the shape model to the individual image. The visible region of shape model was projected on each image and the best match between the shape model and the image was confirmed by visual inspection. 


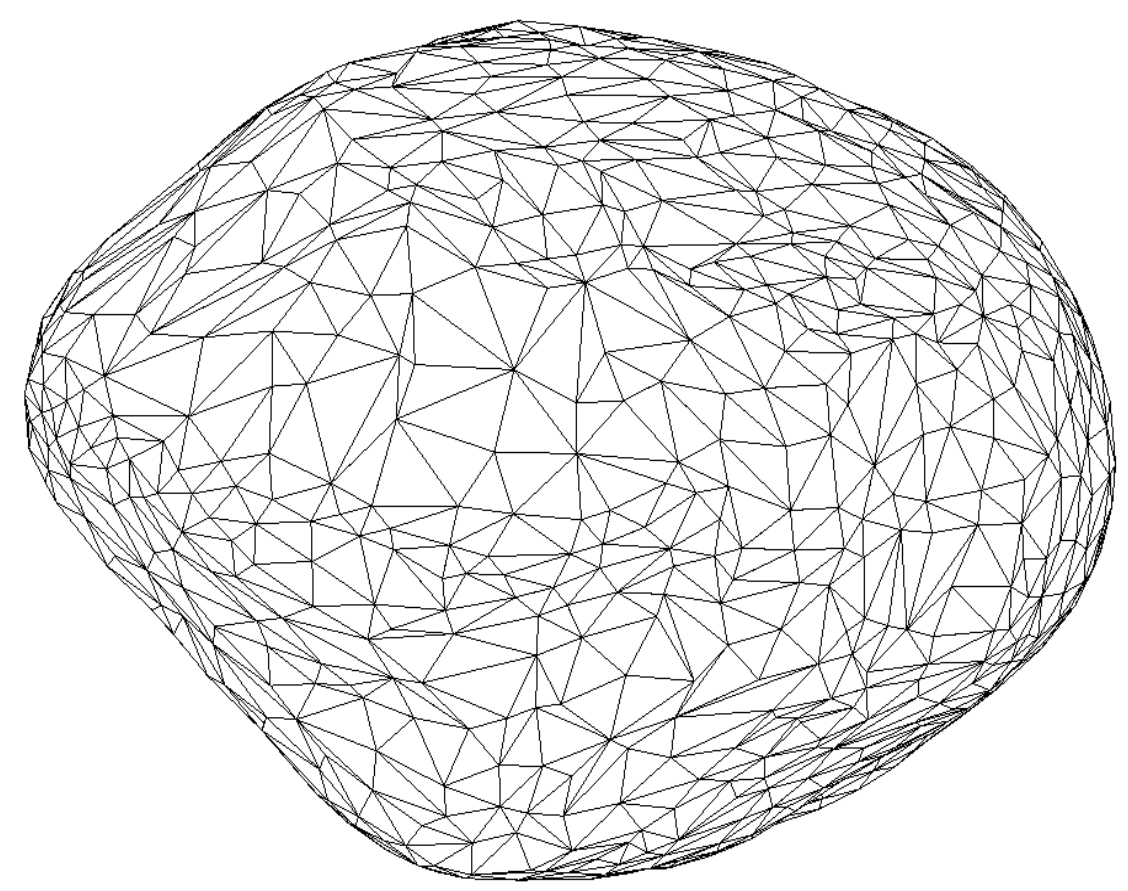

(a)

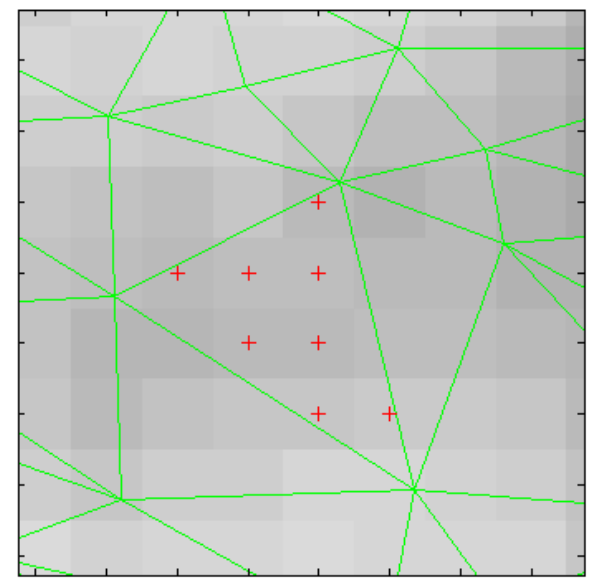

(b)

Figure 4.3: Panel (a) shows the degraded shape model of asteroid Steins covered by 2048 triangular facets. The original shape model of Steins developed by Jorda et al. (2012). Panel (b) shows the example of facet projected on the WAC image of Steins. The facet depicted with green lines is approximately filled with 8 pixels. Pixels are marked by red cross symbol. 
The measured intensity in the facets is converted to the radiance factor. The radiance factor, hereafter denoted as $\mathrm{I} / \mathrm{F}$, is calculated, considering the solar flux at the central wavelength for each OSIRIS filter as well as the distance of the asteroid Steins (2.14 AU) to the Sun at the time of the flyby. For more details about the definition and calculation of $\mathrm{I} / \mathrm{F}$, see chapter 2 .

It is noted that we used the updated calibration information (OSIRIS calibration team, personal communication, November 2014) for all WAC images of asteroid Steins in our analysis. The ratio of old to new absolute calibration factor is multiplied to the brightness extracted from the images.

\subsection{Fitting algorithm}

In order to retrieve the optimum values of the Hapke parameters from both the diskintegrated and the disk-resolved $\mathrm{I} / \mathrm{F}$ values. A direct search method for the fitting is applied. The method was developed according to the Nelder-Mead simplex algorithm (Lagarias et al., 1998) and is implemented by the fminsearch function in Matlab. The Nelder-Mead algorithm utilizes a geometrical pattern to find the best-fit parameters. A geometric object with $\mathrm{N}+1$ vertices in $\mathrm{N}$-dimensions is called 'simplex'. A starting simplex is shaped from $\mathrm{N}+1$ test points. In each iteration, the vertex of simplex has been altered to examine the parameters by analyzing points. A new test point should take the place of the worst one based on the algorithm. The method needs a set of initial parameters as a start point. The initial parameters are produced by a random number generator to stay away from bad starting points, resulting in local solution.

The fminsearch technique searches in the given parameter space to minimize the sum of squares of deviation between the measured and modeled data, known as the norm of residuals, notwithstanding that the minimization is often performed in terms of $\chi^{2}$. The definition of $\chi^{2}$ that we used here is given by,

$$
\chi^{2}=\sum_{n} \frac{\left(I / F_{n, \text { measured }}-I / F_{n, \text { modeled }}\right)^{2}}{\left(I / F_{n, \text { measured }}\right)^{2}}
$$

where $\mathrm{I} / \mathrm{F}_{n, \text { measured }}$ and $\mathrm{I} / \mathrm{F}_{n, \text { modeled }}$ refer to the measured $\mathrm{I} / \mathrm{F}$ of $\mathrm{n}$ equivalent data points from the images and the modeled $\mathrm{I} / \mathrm{F}$ of $\mathrm{n}$ data points predicted from Hapke's equation. The goodness of the modeling is reported by the root mean square (RMS) in percent, where the RMS value returns the square root of $\chi^{2}$.

\subsection{Disk-integrated Reflectance}

\subsubsection{Disk-integrated phase function}

In order to build the disk-integrated phase function of asteroid Steins, we integrated the flux from the images considering a certain threshold flux. The threshold is determined based on the visible disk of the asteroid above noise level. The cross-section of the visible disk of the asteroid is calculated accurately, using the shape model of Steins.

Figure 4.4 displays the disk-integrated phase function of Steins from WAC F17 images at $\lambda=631.6 \mathrm{~nm}$ between $\alpha=0.37^{\circ}$ to $\alpha=137.25^{\circ}$. The data points near the zero phase angle 
$\left(0.37^{\circ}\right)$ allow an estimation of the geometric albedo of the asteroid directly from the diskintegrated phase function. We extrapolate the data to zero phase angle and reached an estimate of $0.38 \pm 0.01$ for the geometric albedo $A_{p}$ of asteroid Steins at $\lambda=631.6 \mathrm{~nm}$. This value agrees within the uncertainties with the one reported by Keller et al. (2010) to be $0.40 \pm 0.01$ at the same wavelength. The Bond albedo $\mathrm{A}_{B}$ is also calculated at the wavelength of $631.6 \mathrm{~nm}$, employing the expression $\mathrm{A}_{B}=\mathrm{A}_{p} \mathrm{q}$, where $\mathrm{q}$ is defined as the phase integral. We were able to calculate the phase integral (equation 2.55 in chapter 2) for the disk-integrated phase function of Steins constructed from WAC F17 images at $\lambda=631.6 \mathrm{~nm}$ thanks to the proper phase angle coverage including also large phase angles. The value for the Bond albedo of asteroid Steins at $631.6 \mathrm{~nm}$ is $0.21 \pm 0.01$.

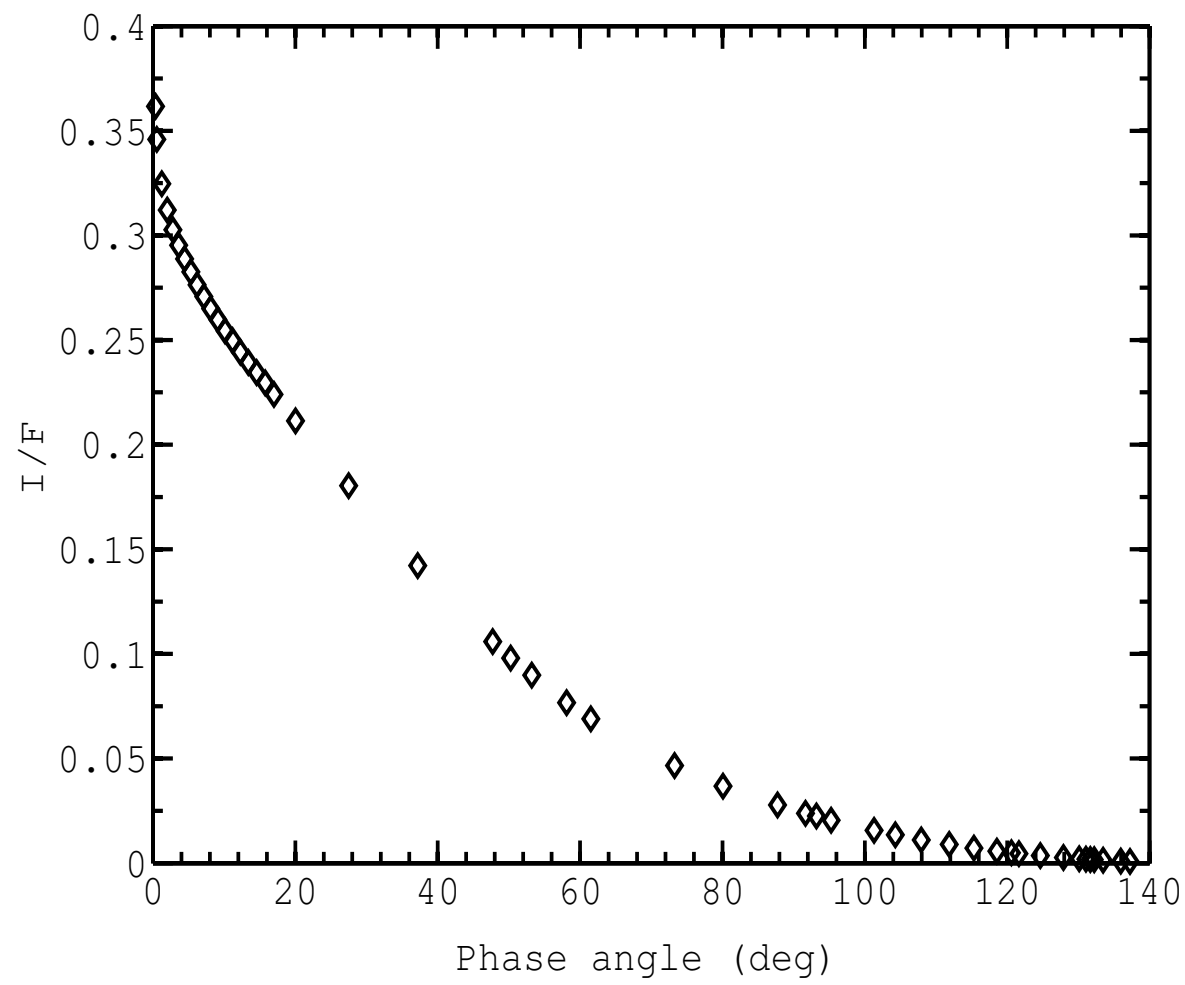

Figure 4.4: Disk-integrated phase function of Steins measured from OSIRIS WAC F17 images at $\lambda=631.6 \mathrm{~nm}$. The uncertainty of the absolute radiometric calibration is reported by OSIRIS calibration team to be about $1 \%$ for all filters. The error bar of data points are not displayed in the figure because they have the same size as the symbol.

\subsubsection{Hapke disk-integrated modeling}

In this section, we used the Hapke disk-integrated equation to model the disk-integrated phase function, I/F with respect to the phase angle, of Steins in different wavelengths. The data we used in our disk-integrated evaluation of Steins are summarized in Table 4.1.

The only disk-integrated phase function of Steins with a wide phase angle coverage is the one constructed from WAC F17 images $(631.6 \mathrm{~nm})$ and depicted in Figure 4.4. For this reason, we first started the fitting procedure with this phase function. As discussed 
Table 4.1: Characteristics of OSIRIS WAC images employed in the disk-integrated analysis of asteroid Steins. The central wavelength for the filters is shown by $\lambda_{c}$ and the time period by UTC.

\begin{tabular}{cccccc}
\hline Camera & Filter Name & $\lambda_{c}(\mathrm{~nm})$ & UTC & Number of images & Phase angle (o) \\
\hline WAC & F51 & 295.9 & $18: 37: 25-18: 47: 53$ & 17 & $20.95-132.25$ \\
WAC & F61 & 309.7 & $18: 37: 27-18: 47: 58$ & 12 & $21.92-132.33$ \\
WAC & F71 & 325.8 & $18: 37: 30-18: 48: 08$ & 13 & $23.32-132.48$ \\
WAC & F81 & 335.9 & $18: 37: 40-18: 48: 11$ & 10 & $28.31-132.52$ \\
WAC & F13 & 375.6 & $18: 37: 42-18: 48: 17$ & 14 & $29.61-132.61$ \\
WAC & F14 & 388.4 & $18: 37: 44-18: 48: 19$ & 14 & $30.38-132.64$ \\
WAC & F15 & 375.6 & $18: 38: 21-19: 25: 58$ & 16 & $52.25-139.60$ \\
WAC & F16 & 590.7 & $18: 38: 58-20: 09: 56$ & 16 & $74.01-140.48$ \\
WAC & F17 & 631.6 & $18: 36: 22-18: 59: 22$ & 50 & $0.37-137.25$ \\
\hline
\end{tabular}

in chapter 2, it is not very reliable to constrain the roughness parameter $\bar{\theta}$ from the diskintegrated phase function. Therefore, we fixed $\bar{\theta}=25^{\circ}$ as estimated from the average of all best-fit values of $\bar{\theta}$ of small bodies. During optimization, fit parameters were the Hapke parameters $\omega, \mathrm{B}_{0}$, h and $\mathrm{g}$. The Best-fit Hapke parameters of Steins from the diskintegrated evaluation at $631.6 \mathrm{~nm}$ are $\omega=0.61 \pm 0.03, \mathrm{~B}_{0}=0.65 \pm 0.10, \mathrm{~h}=0.026 \pm 0.019$, and $\mathrm{g}=-0.30 \pm 0.03$.

For the Hapke disk-integrated modeling of Steins in other wavelengths sufficient data are not available at low phase angles. Hence, we fixed opposition surge parameters, $\mathrm{B}_{0}=0.65$ and $\mathrm{h}=0.026$ modeled from the phase function of Steins from WAC F17 images $(631.6 \mathrm{~nm})$. The results of Hapke modeling for the disk-integrated phase function of Steins in different wavelengths are tabulated in Table 4.2 and depicted in Figure 4.5.

We believe that the g-parameter can be constrained by the disk-integrated data of Steins in all filters, except the one from WAC F16 (590.7 nm) and WAC F15 (375.6 $\mathrm{nm}$ ): based on the Helfenstein and Veverka (1989) study, the asymmetry factor $\mathrm{g}$ in the particle phase function can be retrieved using data from small and large phase angles together. Accordingly, since for the WAC F16 and WAC F15 images, the data points are not available at small phase angles the resulting g-parameters -0.16 for WAC F16 at 590.7 $\mathrm{nm}$ and -0.22 for WAC F15 at $375.6 \mathrm{~nm}$ are not well-founded from our evaluation.

\subsection{Disk-Resolved Reflectance evaluation}

\subsubsection{Minnaert modeling}

We fit the Minnaert model to the disk-resolved images of Steins. The Minnaert equation is $I / F(i, e, \alpha)=A\left(\mu_{0}\right)^{k} \mu^{k-1}$ as described in chapter 2 and contains two parameters $\mathrm{k}$ and A. Both Minnaert parameters are a function of phase angle and wavelength. In order to obtain the Minnaert parameters, we used the linear version of Minnaert function as follow,

$$
\log ((I / F) \mu)=k \log \left(\mu_{0} \mu\right)+\log A
$$


Table 4.2: Hapke model fit to disk-integrated data of Steins with a single-term HG function. Values in square brackets were held constant in the course of fitting.

\begin{tabular}{lcccccccc}
\hline Camera & Filter id & $\lambda_{c}(\mathrm{~nm})$ & $\omega$ & $\mathrm{B}_{0}$ & $\mathrm{~h}$ & $\mathrm{~g}$ & $\bar{\theta}$ & RMS $(\%)$ \\
\hline WAC & F17 & 631.6 & 0.615 & 0.56 & 0.020 & -0.30 & {$\left[25^{\circ}\right]$} & 2.0 \\
WAC & F16 & 590.7 & 0.578 & {$[0.56]$} & {$[0.020]$} & -0.16 & {$\left[25^{\circ}\right]$} & 2.7 \\
WAC & F15 & 375.6 & 0.603 & {$[0.56]$} & {$[0.020]$} & -0.22 & {$\left[25^{\circ}\right]$} & 4.1 \\
WAC & F14 & 388.4 & 0.486 & {$[0.56]$} & {$[0.020]$} & -0.31 & {$\left[25^{\circ}\right]$} & 1.2 \\
WAC & F13 & 375.6 & 0.449 & {$[0.56]$} & {$[0.020]$} & -0.31 & {$\left[25^{\circ}\right]$} & 1.2 \\
WAC & F81 & 335.9 & 0.368 & {$[0.56]$} & {$[0.020]$} & -0.35 & {$\left[25^{\circ}\right]$} & 0.9 \\
WAC & F71 & 325.8 & 0.376 & {$[0.56]$} & {$[0.020]$} & -0.33 & {$\left[25^{\circ}\right]$} & 1.3 \\
WAC & F61 & 309.7 & 0.362 & {$[0.56]$} & {$[0.020]$} & -0.33 & {$\left[25^{\circ}\right]$} & 0.7 \\
WAC & F51 & 295.9 & 0.308 & {$[0.56]$} & {$[0.020]$} & -0.34 & {$\left[25^{\circ}\right]$} & 1.4 \\
\hline
\end{tabular}

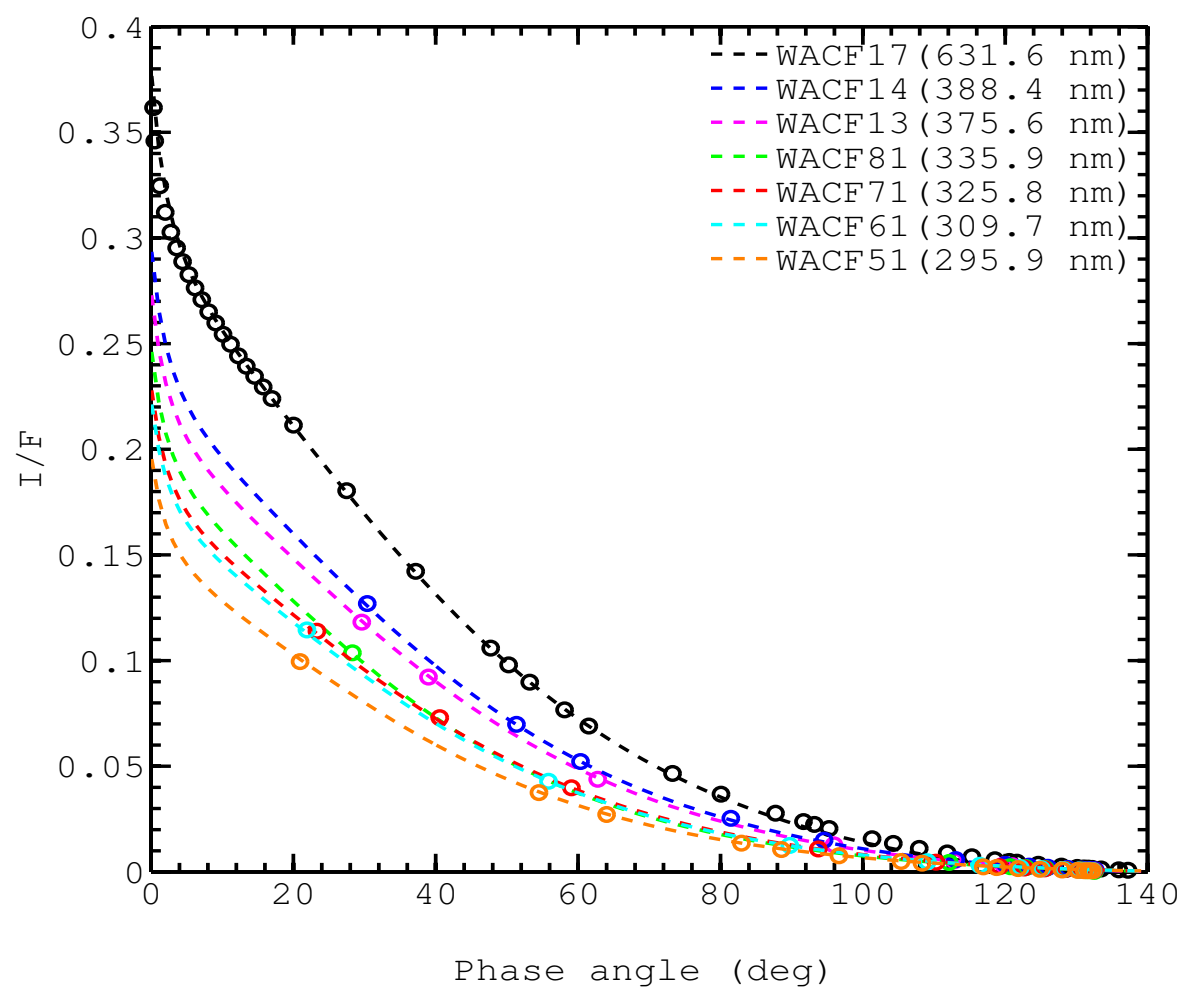

Figure 4.5: Disk-integrated phase function of Steins measured from OSIRIS WAC images in 7 filters. The dash lines display the Hapke disk-integrated fit. The data points from WAC F16 (590.7 nm) and WAC F15 (375.6 nm) as well as the corresponding Hapke fit are not included, since their small data-set are not sufficient to constrain the Hapke parameters. 
The fitting was implemented in nonlinear least squares sense. We do not include the $\mathrm{I} / \mathrm{F}$ data for the incidence and emission angles greater than $70^{\circ}$ because of the inaccuracy of co-registration towards the limb.

The goodness-of-fit plots for the Minnaert fit are displayed in Figure 4.6. The modeled versus measured reflectance plot indicates the overall scatter is rather uniform (Figure 4.6a). However, the plot of the ratio of measured/modeled I/F with respect to incidence angle shows that the deviation versus incidence angle notably increases for $\mathrm{i}>60^{\circ}$ (Figure 4.6p). The scatter along emission angle is also rather high: further analysis shows that this behavior is caused by the images with large phase angles. This is also seen in the relatively high deviation for phase angle $\alpha>50^{\circ}$, which shows the ratio between measured $\mathrm{I} / \mathrm{F}$ and modeled I/f with respect to phase angle (Figure 4.6 $)$.

The modeled Minnaert parameters are plotted against phase angles in Figure 4.7 and compiled in Table 4.3. The Modeled Minnaert $\mathrm{k}(\alpha)$ for Steins shows a linear trend until phase angle about $60^{\circ}$. From the weighted linear fit, we determine the Minnaert k coefficient at opposition to be $0.589 \pm 0.004$. This value, being larger than 0.5 , suggests that the surface of Steins is slightly limb-darkened compared to other small bodies.

The behavior of the modeled Minnaert $\mathrm{A}(\alpha)$ can be described by an empirical function as established by Li et al. (2013c),

$$
A(\alpha)=A_{0} 10^{0.4 \beta \alpha}
$$

Where $\beta$ is a phase slope in mag/deg and $\mathrm{A}_{0}$ gives the Minnaert albedo at opposition. We estimated the values of $\mathrm{A}_{0}$ and $\beta$ for Steins to be $0.367 \pm 0.004$ and $0.0158 \pm 0.0006$ $\mathrm{mag} / \mathrm{deg}$, respectively. The value of $\mathrm{A}_{0}$ is almost equivalent to the geometric albedo of Steins $(0.40 \pm 0.01)$, indicating a small opposition surge.

\subsubsection{Hapke disk-resolved modeling}

We applied the five-parameter version of the Hapke disk-resolved model to the data extracted from the WAC images of Steins in nine filters. This formulation of the Hake model is capable to characterize the photometric properties of surface reasonably well while a minimum number of parameters are used for the fit. We do not use the I/F data points for i $>70^{\circ}$ and $\mathrm{e}>70^{\circ}$ to remove the possible effect of uncertainty in the image registration as well as the limitation of Hapke model at large geometric angles i and e.

Following Spjuth et al. (2012), we performed Hapke disk-resolved modeling for a similar data set of images. The information about all images employed in the disk-resolved evaluation are tabulated in Table 4.4.

The data from WAC F17 images $(631.6 \mathrm{~nm})$ cover a wide range of phase angles and it allows to fit the Hapke model by using model parameters as fit parameters. In Table 4.5, the Hapke solutions for the WAC F17 images at $\lambda=631.6 \mathrm{~nm}$ are listed and the corresponding scatter plots are displayed in Figure 4.8. The measured I/F versus modeled I/F diagram shows a satisfactory fit with the linear correlation coefficient of 0.975 . The model RMS is estimated to be $8.6 \%$ and no systematic deviations are seen in the plots of measured/modeled $\mathrm{I} / \mathrm{F}$ with respect to the scattering angles (i, e, $\alpha$ ). However, it is found that scattered data points are presented in the plot of the measured/modeled bidirectional reflectance versus emission angle: they result mostly from the images at larger phase angles $\left(80^{\circ}, 87^{\circ}, 91^{\circ}\right.$, and $\left.95^{\circ}\right)$. The data extracted from the image taken at larger phase 


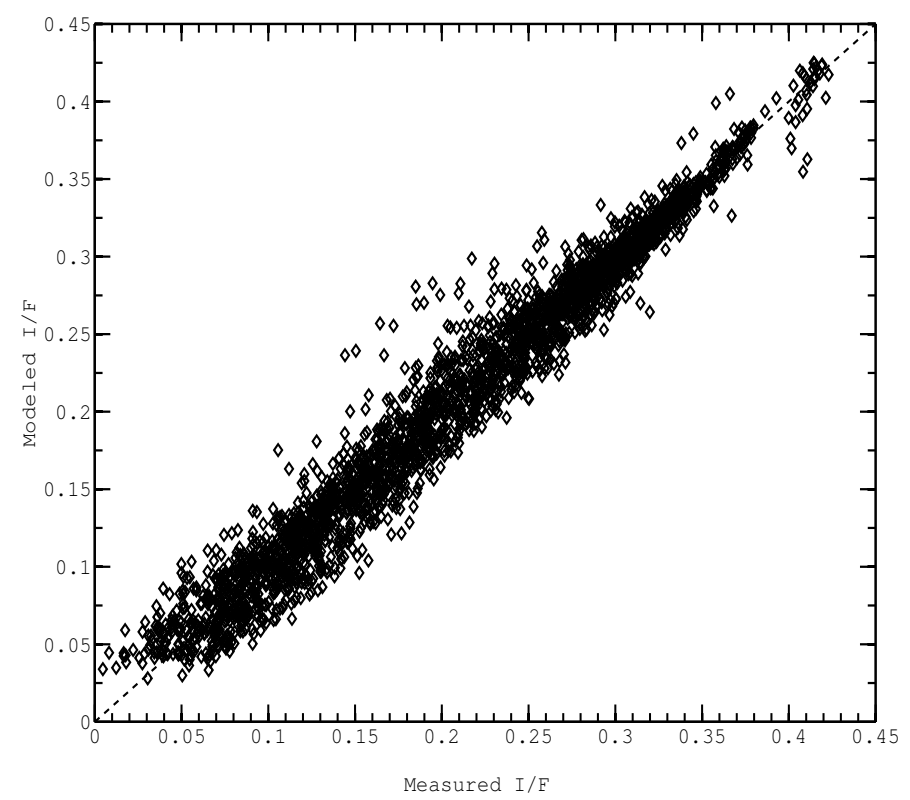

(a)

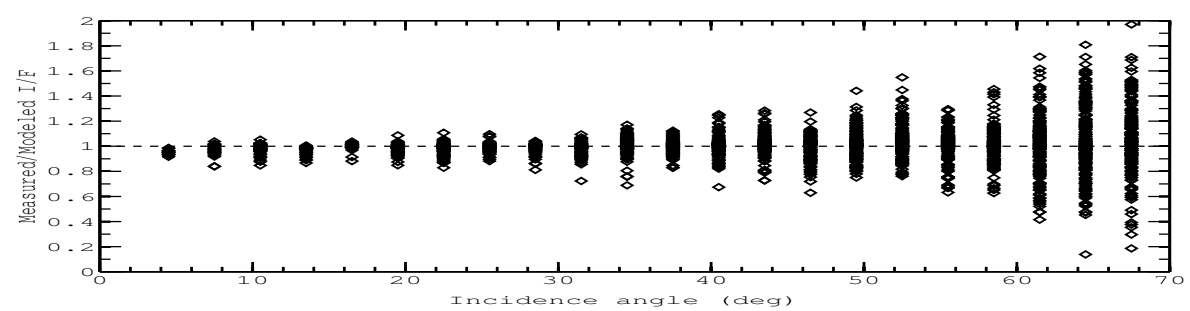

(b)

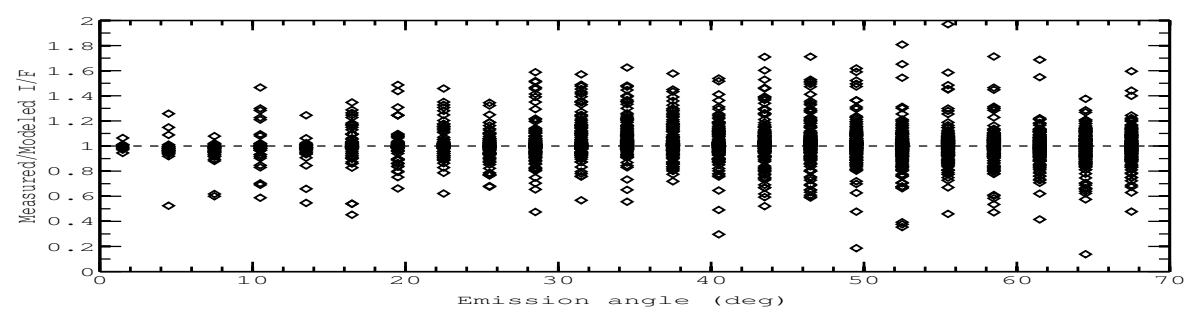

(c)

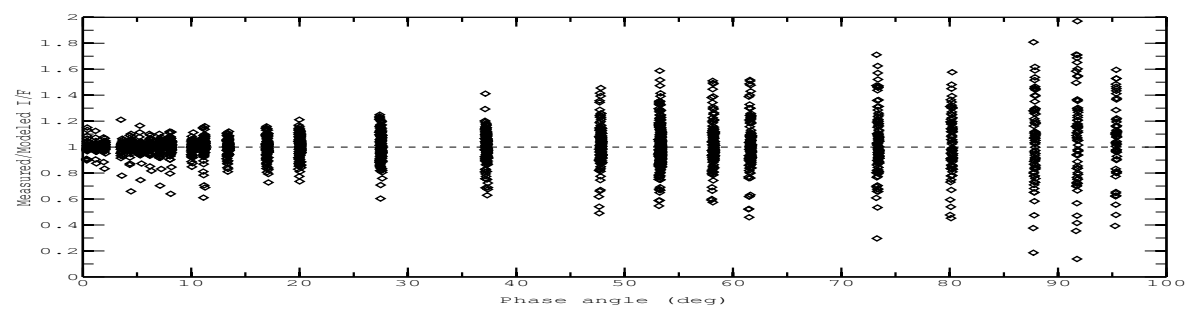

(d)

Figure 4.6: The goodness-of-fit plots for Minnaert modeling of I/F for asteroid Steins from WAC F17 images at $\lambda=631.6 \mathrm{~nm}$. (a) shows the plot of modeled I/F against measured I/F.(b), (c), (d) show the ratio measured I/F to modeled I/F ratio as a function of incidence angle, emission angle, and phase angle, respectively 

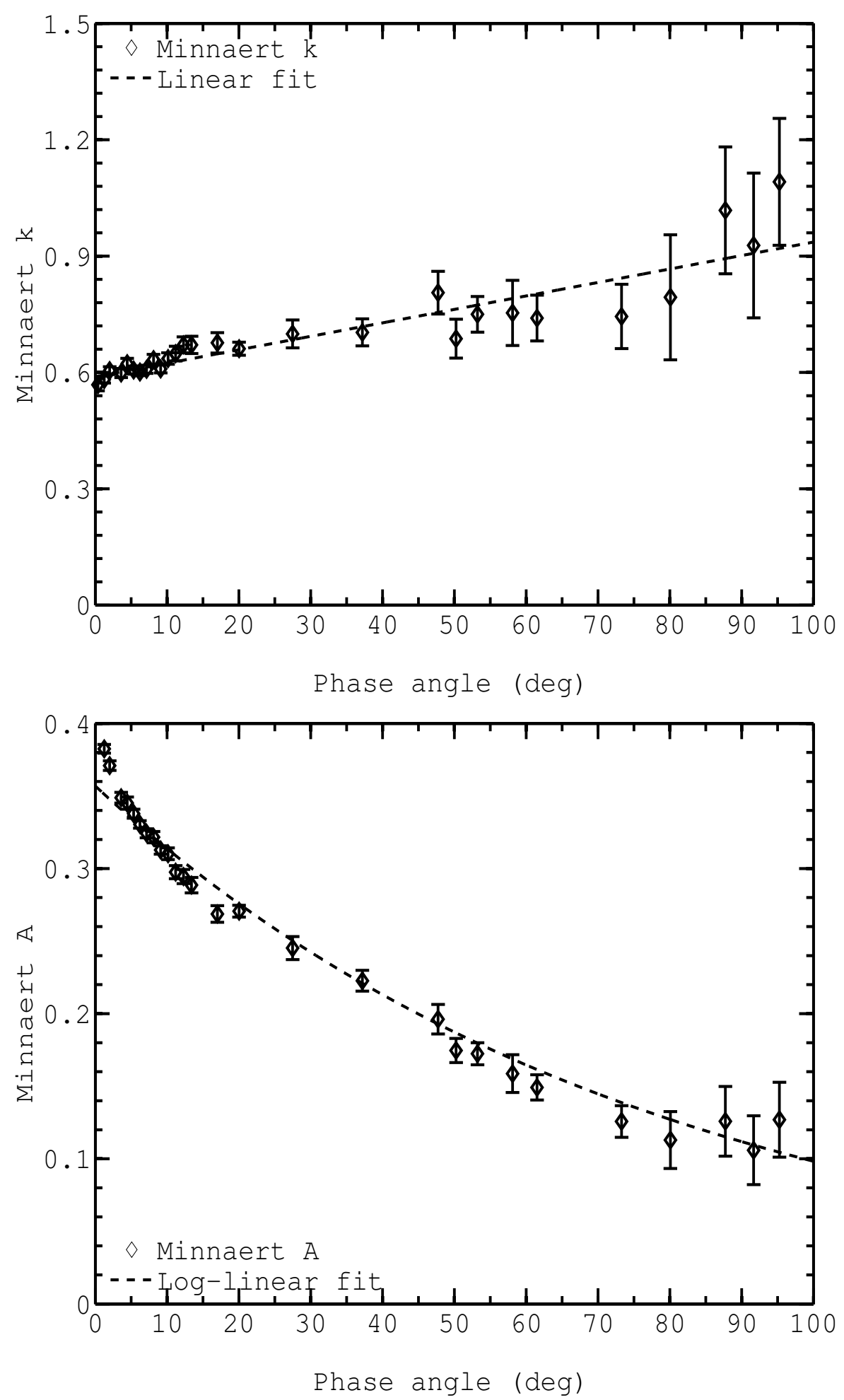

Figure 4.7: The Minnaert parameters obtained for WAC F17 images (631.6 nm) of Steins. Upper panel shows the modeled $\mathrm{k}$ parameter. The dash line is a weighted linear fit to the $\mathrm{k}$ parameter. Lower panel shows Minnaert albedo, A, versus phase angle. 
Table 4.3: Modeled Minnaert parameters for the WAC F17 images of Steins at $\lambda=631.1$ nm.

\begin{tabular}{|c|c|c|c|}
\hline Phase angle (deg) & Minnaert A & Minnaert k & RMS (\%) \\
\hline 0.37 & $0.426 \pm 0.007$ & $0.568 \pm 0.016$ & 4.9 \\
\hline 1.24 & $0.383 \pm 0.003$ & $0.582 \pm 0.009$ & 3.1 \\
\hline 2.00 & $0.371 \pm 0.003$ & $0.605 \pm 0.010$ & 3.4 \\
\hline 3.60 & $0.349 \pm 0.004$ & $0.598 \pm 0.012$ & 4.3 \\
\hline 4.44 & $0.345 \pm 0.004$ & $0.623 \pm 0.014$ & 5.0 \\
\hline 5.33 & $0.338 \pm 0.003$ & $0.61 \pm 0.011$ & 4.2 \\
\hline 6.21 & $0.330 \pm 0.003$ & $0.601 \pm 0.009$ & 3.9 \\
\hline 7.14 & $0.324 \pm 0.003$ & $0.608 \pm 0.011$ & 4.6 \\
\hline 8.11 & $0.322 \pm 0.004$ & $0.633 \pm 0.013$ & 5.6 \\
\hline 9.10 & $0.313 \pm 0.003$ & $0.609 \pm 0.010$ & 4.5 \\
\hline 10.13 & $0.310 \pm 0.004$ & $0.636 \pm 0.015$ & 3.8 \\
\hline 11.19 & $0.298 \pm 0.005$ & $0.649 \pm 0.019$ & 7.0 \\
\hline 12.28 & $0.295 \pm 0.005$ & $0.671 \pm 0.021$ & 6.3 \\
\hline 13.40 & $0.289 \pm 0.005$ & $0.671 \pm 0.022$ & 4.9 \\
\hline 17.00 & $0.269 \pm 0.006$ & $0.676 \pm 0.026$ & 7.3 \\
\hline 20.02 & $0.271 \pm 0.004$ & $0.661 \pm 0.017$ & 7.2 \\
\hline 27.48 & $0.245 \pm 0.008$ & $0.699 \pm 0.036$ & 10.0 \\
\hline 37.19 & $0.223 \pm 0.007$ & $0.703 \pm 0.035$ & 11.2 \\
\hline 47.73 & $0.196 \pm 0.010$ & $0.806 \pm 0.055$ & 11.4 \\
\hline 50.24 & $0.175 \pm 0.008$ & $0.687 \pm 0.050$ & 11.7 \\
\hline 53.22 & $0.172 \pm 0.008$ & $0.750 \pm 0.046$ & 12.9 \\
\hline 58.10 & $0.159 \pm 0.013$ & $0.754 \pm 0.084$ & 12.6 \\
\hline 61.51 & $0.149 \pm 0.009$ & $0.740 \pm 0.059$ & 14.1 \\
\hline 73.28 & $0.126 \pm 0.011$ & $0.744 \pm 0.083$ & 17.9 \\
\hline 80.08 & $0.113 \pm 0.020$ & $0.794 \pm 0.161$ & 18.3 \\
\hline 87.74 & $0.126 \pm 0.024$ & $1.02 \pm 0.164$ & 24.3 \\
\hline 91.67 & $0.106 \pm 0.024$ & $0.928 \pm 0.187$ & 25.7 \\
\hline 95.26 & $0.1269 \pm 0.026$ & $1.091 \pm 0.164$ & 22.6 \\
\hline
\end{tabular}

Table 4.4: Characteristics of OSIRIS WAC images employed in the disk-resolved analysis of Steins.

\begin{tabular}{cccccc}
\hline Camera & Filter Name & $\lambda_{c}(\mathrm{~nm})$ & Number of images & UTC & Phase angle (o) \\
\hline WAC & F17 & 631.6 & 20 & $36: 22-39: 49$ & $0.37-95$ \\
WAC & F16 & 590.7 & 5 & $33: 59-38: 59$ & $8-74$ \\
WAC & F15 & 572.1 & 6 & $34: 13-39: 00$ & $8-74$ \\
WAC & F14 & 388.4 & 8 & $34: 12-39: 14$ & $8-82$ \\
WAC & F13 & 375.6 & 7 & $34: 11-38: 39$ & $8-63$ \\
WAC & F81 & 335.9 & 5 & $34: 09-37: 41$ & $9-29$ \\
WAC & F71 & 325.8 & 7 & $34: 07-38: 33$ & $9-59$ \\
WAC & F61 & 309.7 & 5 & $34: 21-38: 28$ & $9-57$ \\
WAC & F51 & 295.9 & 9 & $34: 03-39: 31$ & $10-88$ \\
\hline
\end{tabular}


angles are most affected by unresolved and resolved shadows. These data points are also displayed a small systemic trend in the modeled to measured I/F diagram. It is noted that the removal of these data points did neither improve the RMS residuals of fit nor the linear correlation coefficient.

Table 4.5: Hapke model fit to disk-resolved data of Steins derived from WAC images in F17 filter $(\lambda=631.6 \mathrm{~nm})$.

\begin{tabular}{lcccccccc}
\hline $\begin{array}{l}\text { Filter } \\
\text { id }\end{array}$ & $\begin{array}{c}\text { Pixel scale } \\
\text { range }(\mathrm{m} / \mathrm{px})\end{array}$ & $\omega$ & $B_{0}$ & $\mathrm{~h}$ & $\mathrm{~g}$ & $\bar{\theta}\left(^{\circ}\right)$ & $\mathrm{RMS}(\%)$ & $\mathrm{Ap}$ \\
\hline F17 & $131-81$ & 0.618 & 0.70 & 0.024 & -0.313 & 27 & 8.6 & 0.41 \\
& & \pm 0.002 & \pm 0.02 & \pm 0.002 & \pm 0.003 & \pm 1 & & \\
\hline
\end{tabular}

We continued the Hapke evaluation for data from eight more filters. The opposition surge parameters, $B_{0}$ and $h$ were fixed, because of the lack of images at small phase angles. We also fixed the asymmetry factor $\mathrm{g}$ as constrained from disk-integrated analysis, because the number of disk-resolved images are less and the phase angle coverage is narrower compared to the disk-integrated one. Apart from the g-parameter of WAC F16 $(590.7 \mathrm{~nm})$ and WAC F15 $(572.1 \mathrm{~nm})$ which were not constrained from the disk-integrated evaluation and they were set to -0.31 . The modeled Hapke parameters are summarized in Table 4.6.

Table 4.6: The best-fit values of the Hapke parameters modeled from disk-resolved analysis with $B_{0}=0.70$ and $h=0.024$ in the wavelength range of $295.9 \mathrm{~nm}$ to 590.7 . The asymmetry factor $\mathrm{g}$ is fixed based on the best-fit value of the disk-integrated evaluation. The geometric albedo is estimated from the Hapke parameters according to equation 2.54 in chapter 2 and listed in column $A_{p}$.

\begin{tabular}{cccccccc}
\hline Camera & Filter ID & $\lambda_{c}(\mathrm{~nm})$ & $\omega$ & $\mathrm{g}$ & $\bar{\theta}$ & $\mathrm{RMS}(\%)$ & $\mathrm{Ap}$ \\
\hline WAC & F16 & 590.7 & $582 \pm 0.009$ & {$[-0.31]$} & $28 \pm 0.5$ & 11 & 0.38 \\
WAC & F15 & 572.1 & $0.607 \pm 0.005$ & {$[-0.31]$} & $28 \pm 0.4$ & 12 & 0.40 \\
WAC & F14 & 388.4 & $0.501 \pm 0.003$ & {$[-0.31]$} & $30 \pm 0.4$ & 10 & 0.32 \\
WAC & F13 & 375.6 & $0.465 \pm 0.003$ & {$[-0.31]$} & $31 \pm 0.5$ & 11 & 0.29 \\
WAC & F81 & 335.9 & $0.383 \pm 0.004$ & {$[-0.35]$} & $32 \pm 0.6$ & 10 & 0.27 \\
WAC & F71 & 325.8 & $0.376 \pm 0.003$ & {$[-0.33]$} & $29 \pm 0.5$ & 14 & 0.25 \\
WAC & F61 & 309.7 & $0.371 \pm 0.003$ & {$[-0.32]$} & $32 \pm 0.8$ & 11 & 0.24 \\
WAC & F51 & 295.9 & $319 \pm 0.003$ & {$[-0.33]$} & $30 \pm 0.7$ & 10 & 0.21 \\
\hline
\end{tabular}

The single scattering albedo SSA and the modeled geometric albedo derived from Hapke modeling are plotted as a function of wavelength in Figure 4.9. The SSA acts like an universal scaling factor to model the data and mimic the spectral slope of the light reflected by the asteroid. The wavelength range below $400 \mathrm{~nm}$ was the Steins's spectrum is not explored before from the ground-based observations (Keller et al., 2010). The spectral slope of SSA is estimated by weighted linear regression to be $(8 \pm 1) \%$ per 100 $\mathrm{nm}$. The linear fit of multi-wavelength geometric albedo shows a slope of 5\% per $100 \mathrm{~nm}$. 


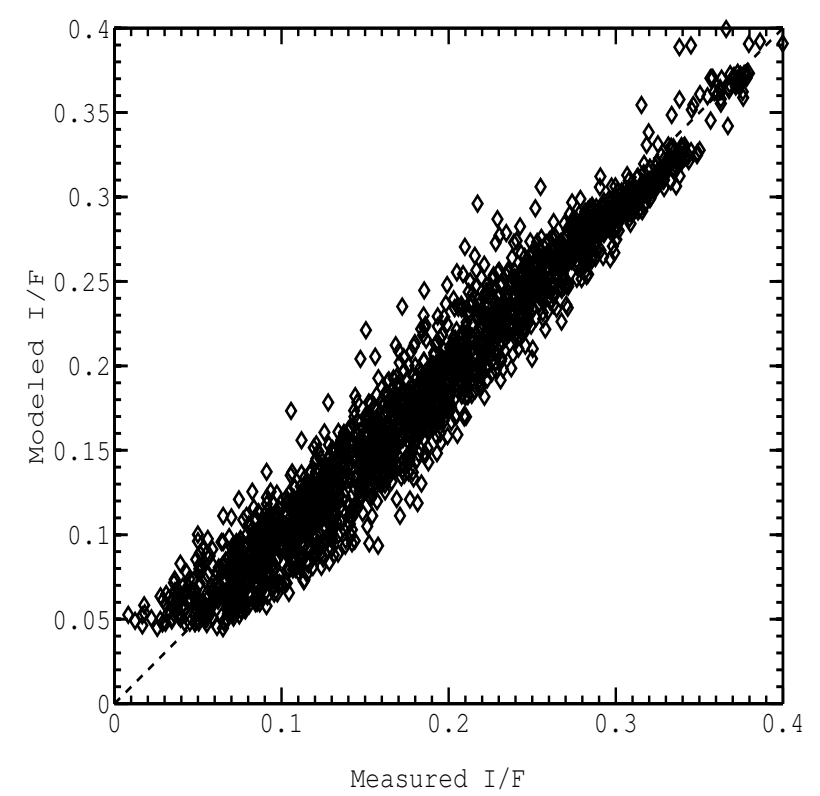

(a)

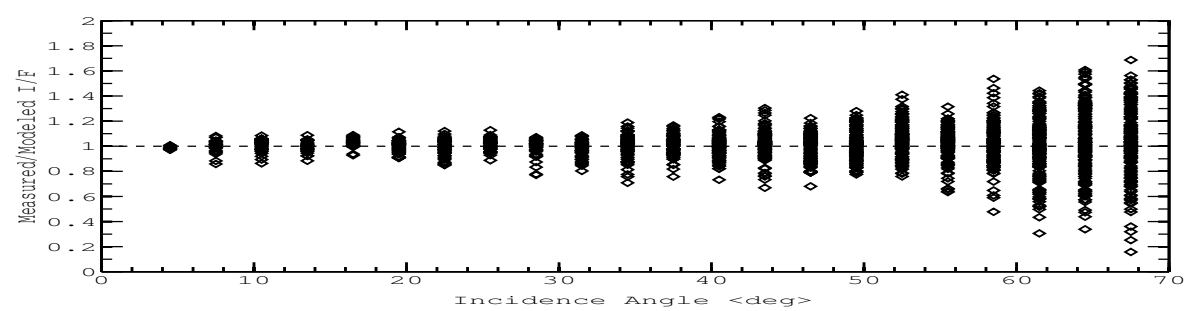

(b)

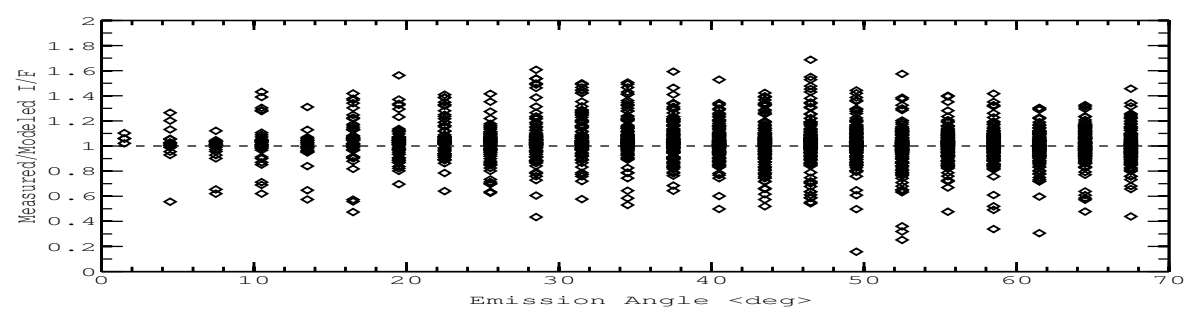

(c)

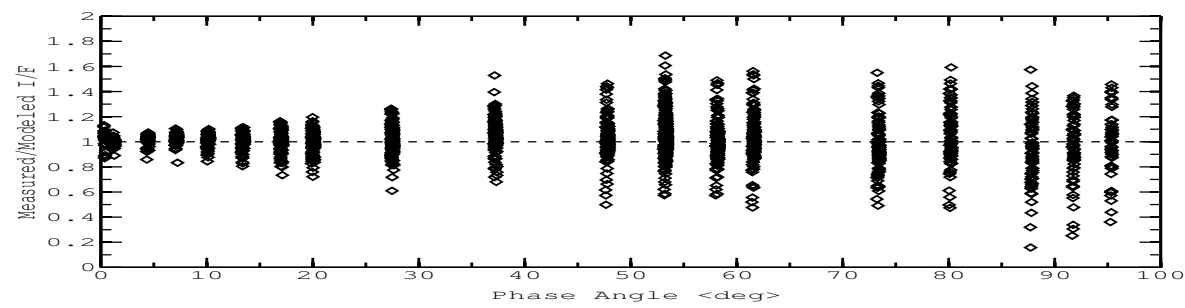

(d)

Figure 4.8: The goodness-of-fit plots for Hapke modeling of asteroid Steins from OSIRIS WAC F17 images at $\lambda=631.6 \mathrm{~nm}$. (a), (b), (c) display from top to bottom the measured $\mathrm{I} / \mathrm{F}$ to modeled I/F ratio as a function of incidence angle, emission angle, and phase angle, respectively. (d) shows the modeled $\mathrm{I} / \mathrm{F}$ versus measured $\mathrm{I} / \mathrm{F}$. 
The SSA may track the geometric albedo. However, it does not mean that their trends exactly match each other, since they are fundamentally different properties. The geometric albedo represents the average reflectance at zero phase angle relative to the same quantity of a perfectly diffuse Lambert disk (Hapke, 1993), whereas SSA is the reflectance of a representative particle, integrated overall all directions. Hence, the SSA is more close to Bond albedo, or spherical albedo, for a planetary body. The difference between an SSA and the geometric albedo is related to the g-parameter of the phase function.

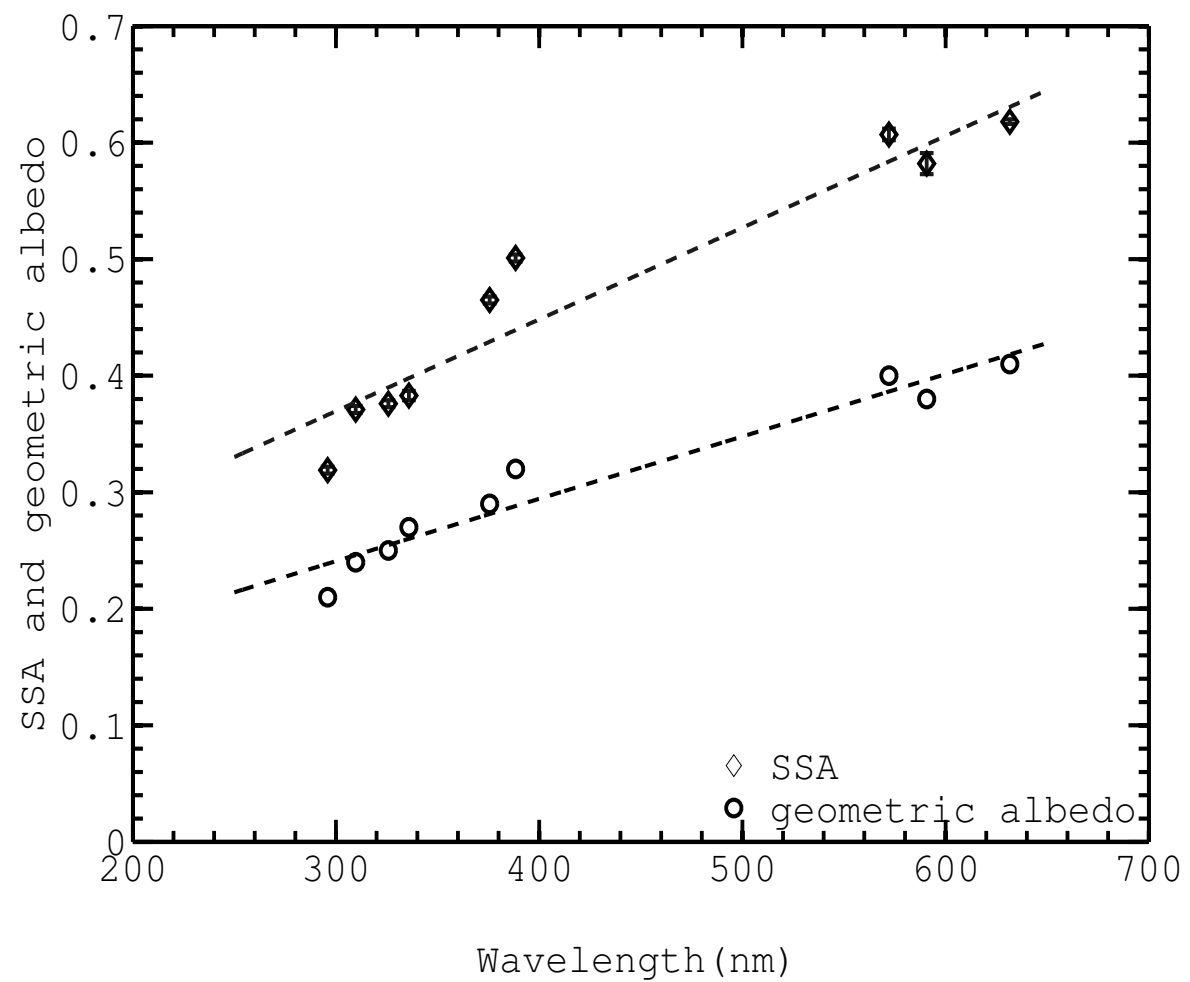

Figure 4.9: The variation of SSA and modeled geometric albedo of asteroid Steins for all OSIRIS WAC filters with respect to wavelength.

As mentioned, the opposition parameters are fixed for eight filters to the best-fit value evaluated from WAC F17 $(631.6 \mathrm{~nm})$ images. Hence, it is not possible to investigate the spectral variation of opposition surge from our data. Considering the variation of the asymmetry factor $g$ with wavelength, the $g$ values are in the small range of -0.35 to -0.31 .

The roughness parameter values also concentrate in a range of $32^{\circ}$ to $27^{\circ}$. However, we note that the values show a trend, i.e. they are decreasing by the wavelength increment. The difference of roughness is just $5^{\circ}$ for all nine wavelengths. Due to the limit in Hapke model (complexity of the model, many intrinsic assumptions and approximations), it is generally hard to constrain the roughness parameter to within a few degrees.

\subsection{Error analysis of the modeled Hapke parameters}

In this section, we describe the bootstrap resampling method to estimate the 1- $\sigma$ error of the modeled Hapke parameters. The determination of the uncertainty for the Hapke 
parameters involves several intricate aspects such as the deficiency of the Hapke model by itself to fully explain the light scattering behavior of planetary surfaces as well as the form of the model with the inter-coupled parameters. The re-sampling analysis is a statistical technique that relies on randomly distributed subsets of data points corresponding to a original set of data points. For instance, this method is employed by Spjuth et al. (2012) for asteroid Steins to find the errorbar of Hapke parameters.

The re-sampling procedure is as follows: First, we find the best-fit value for the Hapke parameters using all data points. Then, in order to determine the uncertainty of these values, we generate a Gaussian random distribution for each $\mathrm{I} / \mathrm{F}$ data point with a mean value at the observed $\mathrm{I} / \mathrm{F}$ and a width of the average error for all $\mathrm{I} / \mathrm{F}$ data points. We set the standard deviation of Gaussian distribution to 0.01 (used in the random generator) which is equivalent to the absolute radiometric calibration uncertainty. The re-sampled I/F data set as a result of the random generator are fitted by the Hapke model to find the new best-fit values of parameters. This series of actions is repeated 1000 times. The standard deviation of the 1000 sample solution of every single parameter is considered a measure for the uncertainty of each Hapke parameter.

The re-sampling procedure can be applied to both disk-integrated and disk-resolved data. However, the parameter uncertainty found from the resampled I/F of the diskresolved data points is rather reliable because of data constraint through the geometrical angles of i, e, $\alpha$. Whereas, the disk-integrated data are restricted only by the phase angle, $\alpha$.

We replicated data points of WAC F17 images $(631.6 \mathrm{~nm})$ when all five Hapke parameters are allowed to change. The re-sampling process is also done for the data points of other filters when three Hapke parameters of $\mathrm{B}_{0}, \mathrm{~h}$, and $\mathrm{g}$ are kept fixed. The resulting uncertainties of modeled Hapke parameters are tabulated with the best-fit values in Table 4.6.

\subsection{Comparison with previous analyses}

In this section we evaluate our approach by comparing our results from the disk-integrated and disk-resolved evaluations of asteroid Steins images with the work of Spjuth et al. (2012). It is noted that Hapke modeling is done only for the disk-resolved data of Steins by Spjuth et al. (2012).

The two main Hapke parameters constrained by Spjuth et al. (2012) for all filters are the SSA and the roughness parameter: the other Hapke parameters $\mathrm{B}_{0}, \mathrm{~h}$ and $\mathrm{g}$ are modeled from WAC F17 $(631.6 \mathrm{~nm})$ images only. The best-fit SSA derived from our analysis are plotted together with the previous result in Figure 4.10. We calculated the reddening slope of SSA spectrum to be $(8 \pm 1) \%$ per $100 \mathrm{~nm}$ which is in agreement with the spectral slope of $(7 \pm 2) \%$ per $100 \mathrm{~nm}$ derived from SSA spectrum reported by Spjuth et al. (2012). It should be noted that the discrepancy seen between the spectral variations of SSA from our evaluation with those derived previously is mostly due to the fact that we used the new calibration parameters for the OSIRIS WAC images.

The opposition surge parameters derived from our analysis of WAC F17 images (631.6 $\mathrm{nm}), \mathrm{B}_{0}=0.70 \pm 0.02$ and $\mathrm{h}=0.024 \pm 0.002$ agrees to some extent with those modeled by Spjuth et al. (2012) to be $\mathrm{B}_{0}=0.60 \pm 0.05$ and $\mathrm{h}=0.027 \pm 0.002$. However, the amplitude 


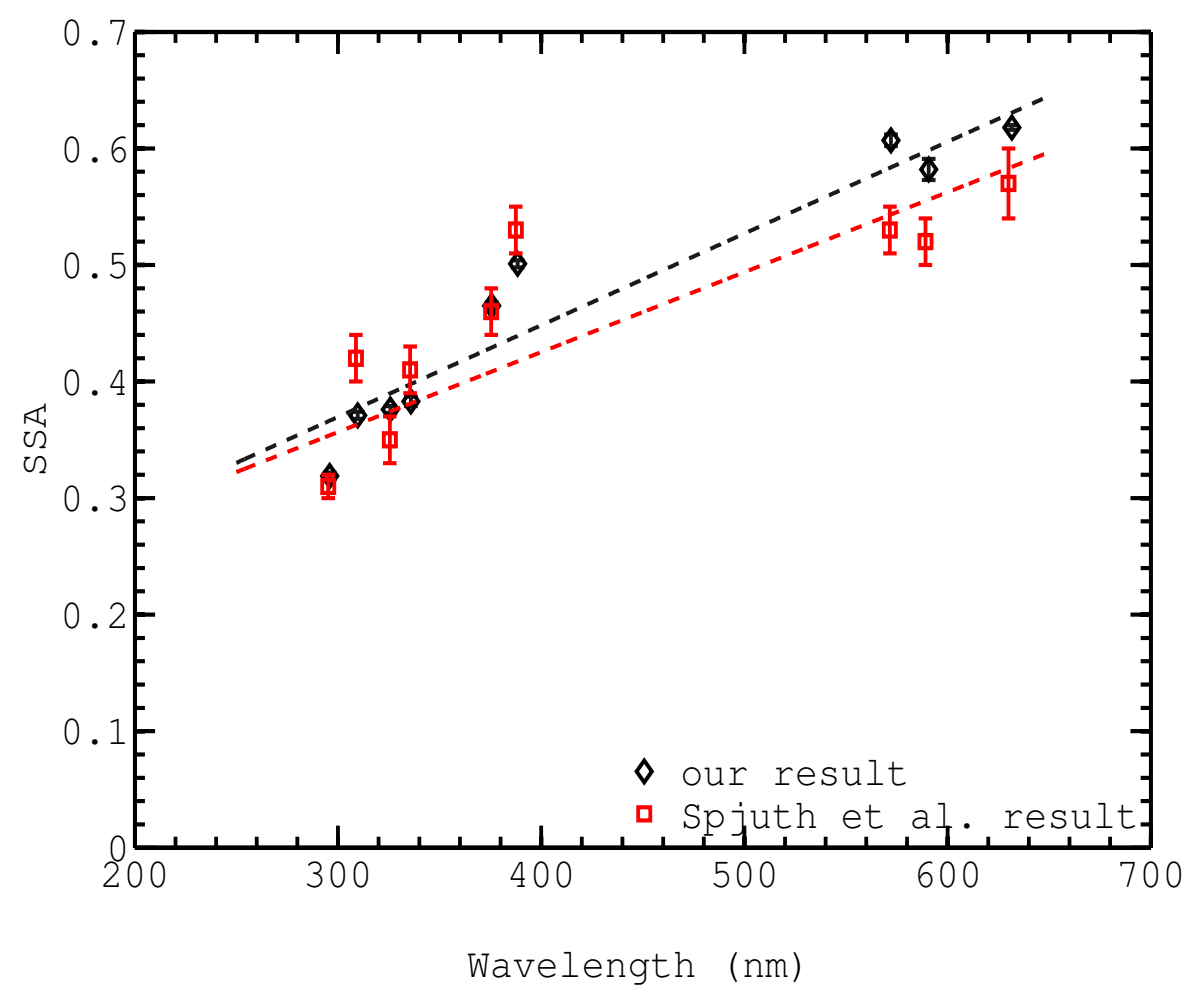

Figure 4.10: Comparison of the modeled single scattering albedo (SSA) variations as a function of wavelength with the results published by Spjuth et al. (2012) for asteroid Steins.

of opposition surge modeled by our evaluation is slightly higher. This small difference is probably caused by using the new image calibration.

The modeled asymmetry factor $\mathrm{g}=-0.313 \pm 0.003$ at $\lambda=631.6 \mathrm{~nm}$ is compatible with the g-parameter constrained by Spjuth et al. (2012) to be $-0.30 \pm 0.01$.

We also modeled the roughness parameter for all nine filters (Figure 4.11). The bestfit value obtained for the F17 filter $(631.6 \mathrm{~nm})$ of WAC images is $27^{\circ} \pm 1^{\circ}$. This value is consistent with the value of Spjuth et al. (2012), $28^{\circ} \pm 2^{\circ}$. The value of the roughness parameter for the other eight filters is also in agreement with the results of Spjuth et al. (2012); it shows a very similar trend of decreasing roughness with wavelength. 


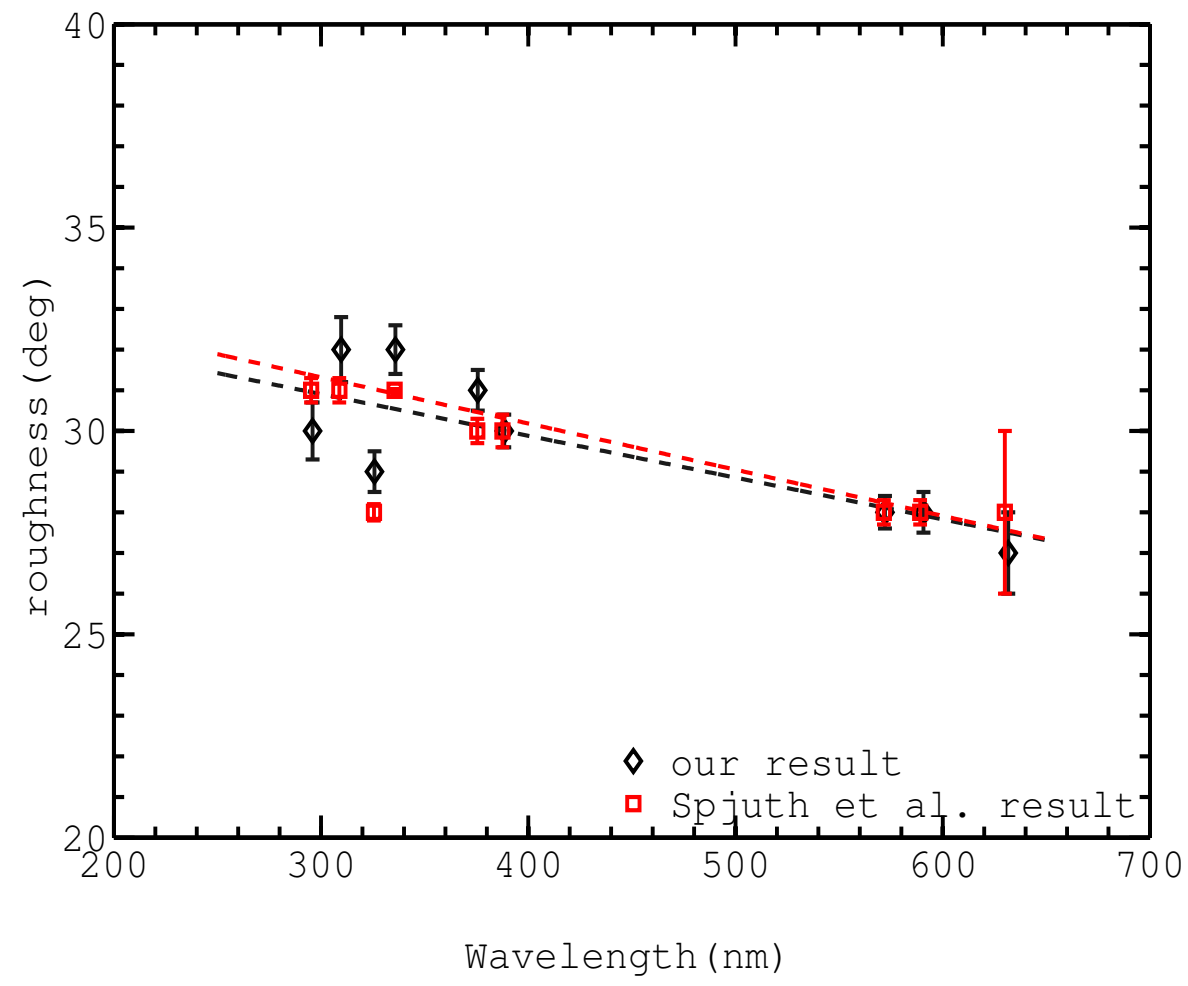

Figure 4.11: Comparison of the modeled roughness for the nine filters of WAC images with those values of roughness constrained by Spjuth et al. (2012) for asteroid Steins. 


\section{Surface Reflectance Analysis of Asteroid (21) Lutetia}

\subsection{Overview of Lutetia's Surface}

The Surface of Lutetia was explored by the nine of the eleven Rosetta instruments (Schulz et al., 2012) during a close flyby in July 2010. Most of what is studied about the surface of Lutetia has been originated in the scientific objectives of four instruments on board the Rosetta. The measurements of the Visible, Infrared Thermal Imaging Spectrometer (VIRTIS) include spectral reflectance and temperature maps in the wavelength range of 0.4 to $3.5 \mu \mathrm{m}$ (Coradini et al., 1999). The ultraviolet spectra of Lutetia has been measured by another spectrograph onboard Rosetta, ALICE instrument (Stern et al., 2011). The thermal fluxes from the Lutetia's surface is also measured by Microwave Instrument (MIRO) onboard Rosetta at infrared, submillimeter, and millimeter wavelengths (Gulkis et al. 2007). The multi-band, visible wavelength images recorded by the OSIRIS camera system (Keller et al., 2007 Sierks et al., 2011;) onboard Rosetta made it possible to scrutinize the surface such as geomorphological, geophysical, and cratering history analyses (Thomas et al., 2012,Massironi et al., 2012, Marchi et al., 2012 Vincent et al., 2012).

The surface of asteroid Lutetia is believed to be a complex assemblage of regions, featuring structures with different geologic histories. The surface is partitioned into six major districts due to different crater density, distinctive surface features and textures. Based on Sierks et al. (2011), the name of these regions are: Archaia (Ac), Baetica(Bt), Etrutia(Et), Narbonesis(Nb), Noricum(Nr), Pannonia(Pa) (Figure 5.1).

Achaia contains plenty of craters and lineaments, however the topography of this region is comparatively undisturbed. According to this fact, Achaia region counts as an old region with 3.4-3.7 Ga of age. However, the two major craters in the Achaia unit, called Nicaea and Burdigala are younger. The depth-to-diameter ratio (d/D) of the Achaia region also provides evidence that it region can be divided into two sub-units (Figure 5.2 ).

The Baetica region contains the most notable feature on Lutetia, known as the North Polar Crater Cluster (NPCC), and its ejecta. Within the NPCC, it is possible to identify three prominent craters with different diameters: Corduba $(34 \mathrm{~km})$, Gades $(21 \mathrm{~km})$, and Hispalis $(14 \mathrm{~km})$. The two smaller craters, Gades and Hispalis, are superimposed on the larger one, Corduba. Except Hispalis, both Corduba and Gades show various features such as depressions, boulders, and landslides. All these phenomena suggest Baetica to be the youngest area on the surface of Lutetia. The age of the central 21-km-diameter crater and its ejecta is estimated to be less than $300 \mathrm{Ma}$ old, the bigger part surrounding the crater which is recognized as thick-layer blanket is considered to be older. 
The Etruria region is difficult to be characterized in view of a geomorphological study as well as age estimation because the flyby geometry resulted in adverse illumination conditions. The major feature recognized in Etruria is a V-shaped structure.

The Narbonensis region is predominantly filled by the Massilia crater, a small part of this region occupied by the Florenita crater. Massilia with $55 \mathrm{~km}$ diameter is the largest crater discerned on Lutetia. The age of the Narbonensis area has been estimated to be likely 1-1.5 Ga (Figure 5.2p).

Noricum has also an high crater density, but it is diverse in morphology including several grooves and groove-like lineaments. The complexity of $\mathrm{d} / \mathrm{D}$ distribution proves the intricacy of Noricum's morphology (Figure 5.2 $\mathrm{k}$ ). The age of the Noricum region is estimated to be 3.4-3.7 Ga, except two sub-regions; the Salona crater is younger than the whole region but not as young as the Patavium crater and its ejecta that is less than 300 Ma old.

Magrin et al., 2012 examined the surface variegation of Lutetia from OSIRS NAC images in the visible blue, far- and near-UV filters, zooming in the Baetica region. They highlighted the probable presence of surface variation on this region which can be interpreted as a possible compositional and/or grain size variety. but, the achievable variegation from these filters is within the uncertainty of their technique.

The surface composition and albedo information of Lutetia is reviewed by Barucci et al. 2012 based on spectral and polarimetric measurement from telescopic observations as well as spectral measurement by VIRITIS.

The measurement of VIRTIS which has been done only on the northern region of Lutetia (Coradini et al., 2011), in the range of 0.25-5.1 $\mu \mathrm{m}$ shows featureless spectra, expect above $3.5 \mu \mathrm{m}$. No sign of olivine or pyroxene (the absorption bands at 1 and $2 \mu \mathrm{m}$ ) are detected in the spectra. The bands are associated with aqueous alternation such as 1.9, 2.7 or $3 \mu \mathrm{m}$ are not seen in the spectra, nor were organic material at 3.3-3.6 $\mu \mathrm{m}$ identified. According to the analysis of Coradini et al. (2011), the spectrum of Lutetia is remarkably flat. The possible chondrites which are matched with the flat and featureless spectrum of Lutetia in the wavelength range of 0.5 to $2.4 \mu \mathrm{m}$ are introduced by Coradini et al. (2011) (Figure 5.3). For instance, a number of carbonaceous chondrites which belong to CM and CI classes (the most primitive groups) as well as several metal-rich carbonaceous chondrites such as $\mathrm{CB}, \mathrm{CH}$, and $\mathrm{CR}$ classes. Due to low reflectance, the primitive one are excluded, while the metal-rich one explain the large density of Lutetia, $3400 \mathrm{~kg} \mathrm{~m}^{-3}$ (Sierks et al., 2011). The spectra of one enstatite chondrite (Eagle EL6) also represent quite well the lack of reddening in the spectrum of Lutetia.

The telescopic spectra mostly cover the whole surface of Lutetia. Those corresponding to the northern hemisphere are depicted in Figure 5.4 and all telescopic spectral observation are tabulated in Table 5.1. The spectral observation in the visible range is divided into two groups, the northern part and the southern part, due to the dissimilar appearance of small features. In the visible spectral range $(0.4-0.9 \mu \mathrm{m})$, several small features at $0.43 \mu \mathrm{m}, 0.51 \mu \mathrm{m}, \sim 0.8 \mu$ mexist. The features at $0.43 \mu \mathrm{m}$ and $0.51 \mu \mathrm{m}$ are reported by Lazzarin et al. (2004) from the observation of the northern part, while these features also appeared in the spectra obtained of the southern part by Lazzarin, M. et al. (2009); Perna, D. et al. (2010); Belskaya et al. (2010). The other small feature at $\sim 0.8-0.82$ (the evidence of Olivine) is mostly seen in the spectra of the southern polar region, except for a faint absorption feature around $0.83 \mu \mathrm{m}$ in the spectrum of northern region as observed 


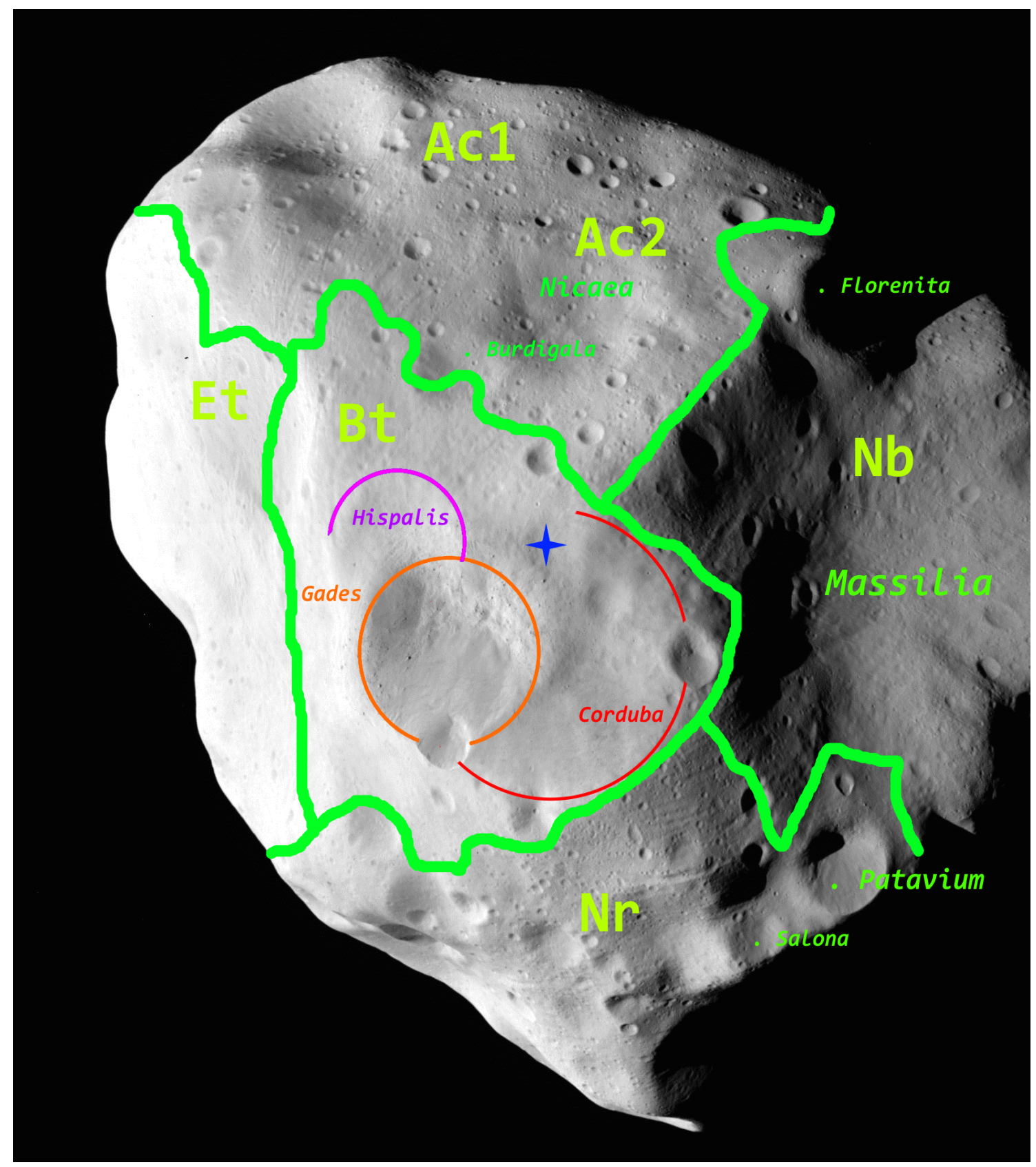

Figure 5.1: The borderlines of regions defined on Lutetia. Ac:Achaia, Nr;Noricum, $\mathrm{Nb}$ :Narbonensis, Bt:Baetica, Et:Etruia (following (Massironi et al., 2012). The official nomenclature of features is approved by IAU in 2011-04-05. The rough position of the north pole is indicated by blue four-point-star. 


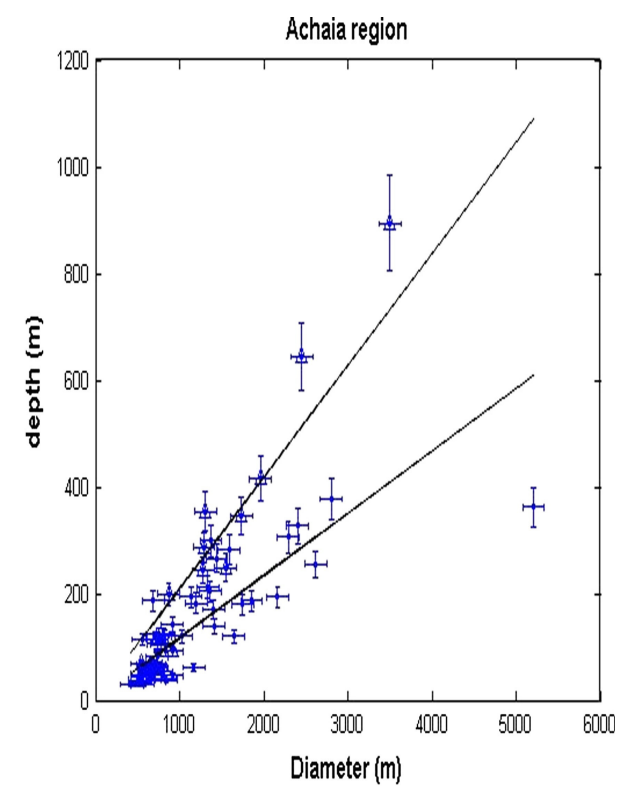

(a) Achaia

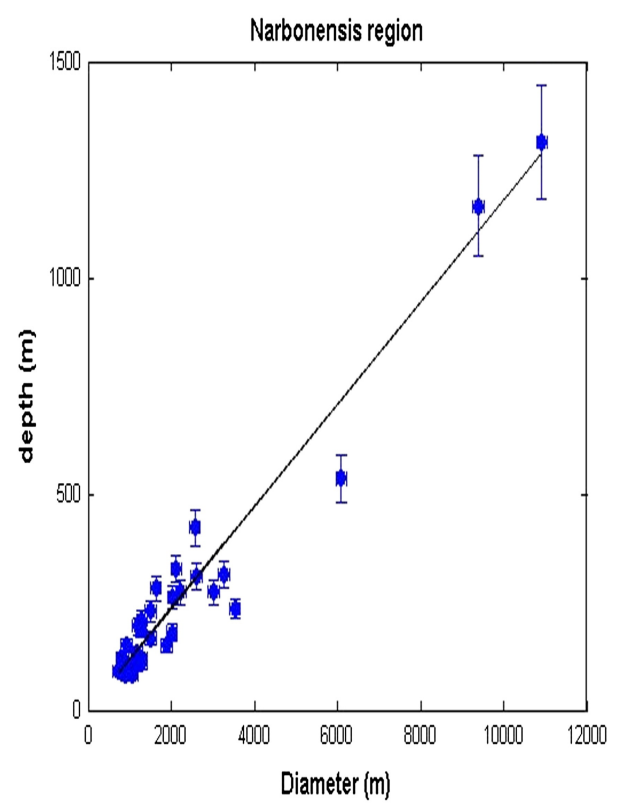

(b) Narbonensis

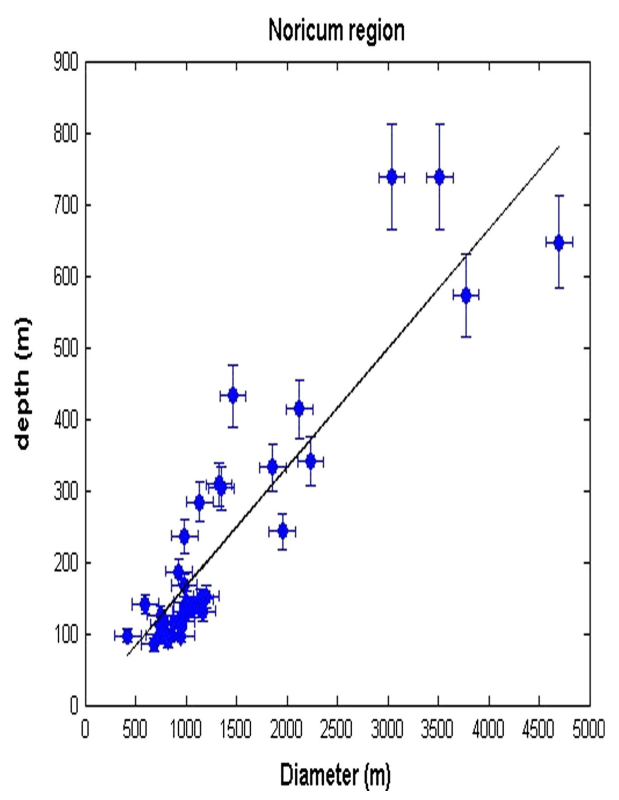

(c) Noricum

Figure 5.2: Plots of depth versus diameter of craters on different regions of asteroid Lutetia studied by Vincent et al. (2012). The solid lines shows the overall trend of data points. It is evident that the Achaia region displays two distinct linear behaviors and the Narbonensis region indicates a noticeable linear trend, whereas the Noricum region behaves very different from the linear fit (adapted from figures 6 and 7 inVincent et al., 2012). 


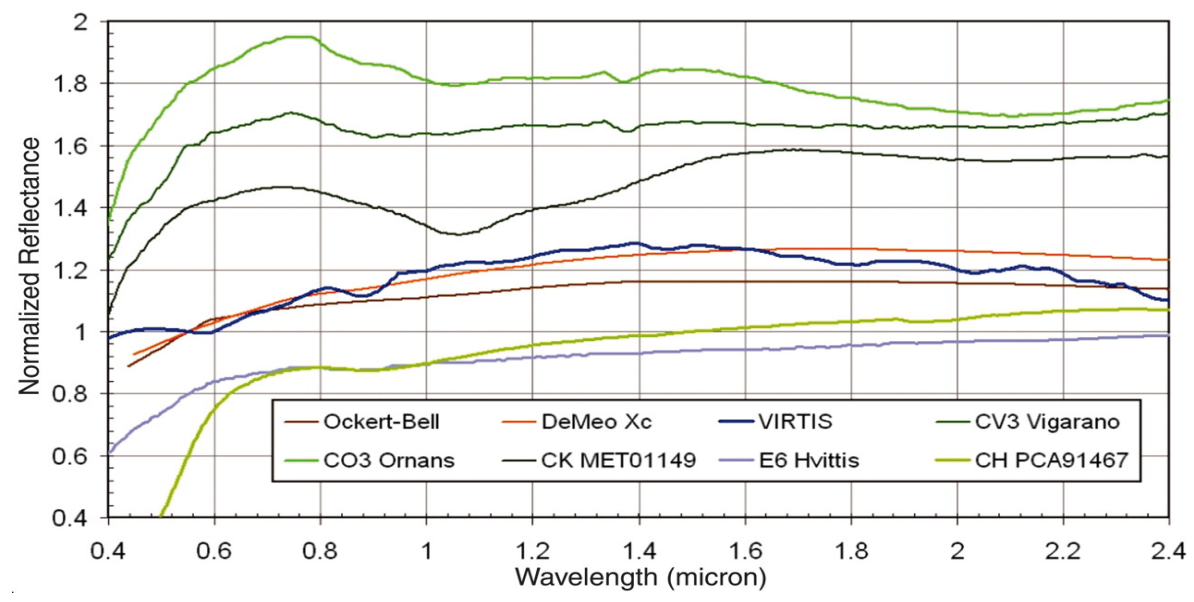

Figure 5.3: The VIRTIS spectrum of Lutetia in the range of 0.5 to $2.4 \mu \mathrm{m}$. The spectrum is compared with the Xc class (DeMeo Xc) and M class (Ockert-Bell), taken from figure 2 in Coradini et al. (2011)

by Belskaya et al. (2010). In the near-infrared range (0.9-2.4 $\mu \mathrm{m}$, the spectral behavior is different for the northern and southern regions of Lutetia. All spectra measured for the southern part have either neutral or slightly negative slopes (blueish), whereas the spectral slope for the northern part is positive (reddish). In the $3 \mu \mathrm{m}$ spectral region, several observations were published by Birlan et al. (2006), Birlan et al. (2010) and Rivkin et al. (2000), Rivkin et al. (2011). The existence of an absorption at $3 \mu \mathrm{m}$ (as a proxy of hydrated minerals) is obvious in the spectra of Lutetia's surface, but it shows various depths over the surface. Those associated with hydrated minerals are related to the southern aspect views, while the absorption in the spectra of northern hemisphere has no sign of water.

In the mid-infrared range, Lutetia is observed by Barucci et al. (2008). The observation was performed over the entire rotation period with the Infrared Spectrograph of the Spitzer space telescope. The Spitzer spectra correspond to the southern/equatorial aspect of Lutetia's surface. They indicate a Christensen peak at $9.3 \mu \mathrm{m}$. However, the presence of the Christensen peak at $9.3 \mu \mathrm{m}$ attributed to carbonaceous chondrites is in conflict with studies such as Vernazza et al. (2011) that found a considerable match between the enstatite chondrite meteorites and Lutetia spectra.

The spectra of Lutetia is also recorded in other range of wavelength from 70 to 205 nm, ultraviolet (UV), by imaging spectrometer onboard Rosetta UV ALICE (Stern et al., 2011). A spectral feature is detected by this instrument between 160 and $180 \mathrm{~nm}$. The physical interpretation of UV spectral feature, however, is not possible because no laboratory data is available at this range. Based on Stern et al. (2011) study, a combination of $77 \%$ EH5 chondrite and $7.7 \%$ from each of these calcium-rich minerals (anorthite), $\mathrm{H}_{2} \mathrm{O}$ frost and $\mathrm{SO}_{2}$ frost is in agreement with the UV spectral feature of Lutetia.

A temperature map of Lutetia is derived from the measurement of MIRO instrument (Gulkis et al., 2012) by studying the near-surface temperature at two wavelengths 190 $\mathrm{GHz}(1.6 \mathrm{~mm})$ and $560 \mathrm{GHz}(0.5 \mathrm{~mm})$. The MIRO beams scanned the northern hemisphere (day-side since it is continually illuminated) and southern hemisphere (night-side since it is not illuminated during the flyby) of Lutetia, indicating physical temperature of the day-side is in the range 197-208 $\mathrm{K}$ and the dark-side in the range 62-72 k. (Gulkis 
et al., 2012) points out that the Lutetia regolith profile which is constrained by MIRO resemble the lunar regolith profile derived by Apollo. This suggests that the same process involves in keeping a regolith in the surface of airless bodies.

A uniform model for the surface layer of Lutetia based on its thermal flux measurements provided by Keihm et al. (2012), using the complementary data from the combination of VIRITS and MIRO instruments. The MIRO evaluation from dark-side of Lutetia supports the VIRTIS day-side finding that Lutetia has a low thermal inertia. The direct VIRTIS evaluation of Lutetia day-side surface shows that the temperature distribution is 5-30 k higher, getting closer to the limbs, compared to the MIRO thermal model based on the smooth surface. As argued by Keihm et al. (2012), the discrepancy increases toward the limbs suggests that the roughness effect needs to be considered into the MIRO thermal model for the understanding of disk-resolved thermal radiant.

Despite these studies, photometric properties of Lutetia have never been examined and the disk-resolved reflectance of the surface was not yet modeled in terms of photometric functions. In this chapter, a complete surface reflectance analysis is presented. We aim to retrieve the light scattering parameters of asteroid Lutetia using disk-integrated and disk-resolved flux as well as scrutinize the surface variations of object.

\subsection{Data extraction}

The method that we utilized to extract information from the observed images are provided in this section. We need the shape model of Lutetia by Jorda et al.(2010) for two purposes. First, in order to calculate the local scattering geometry, we require the shape model which is associated with the SPICE data of Rosetta's flyby distributed by ESA (ftp:// ssols01. esac. esa.int/pub/data/SPICE/ROSETTA/kernels/). Second we need to collect the intensity per facet by co-registering the images of the asteroid with the shape model.

The shape model used for the analysis of OSIRIS NAC images of Lutetia was formed with 45778 triangular facets and shown in Figure 5.5. Each facet is filled with $\sim 100$ pixels in the case of OSIRIS NAC images (Figure 5.5). The sum of all pixel fluxes within each individual facet is used as average radiance.

In the analysis of WAC images, we degraded the shape model $10 \%$ because the original shape model is not compatible with the resolution of WAC images. The problem arises when the size facets are smaller than pixels. The degradation allows us to have statistically proper average number of pixels $(\sim 30)$ within each facet of the degraded shape model. In Figure 5.6 the 10\% degraded shape model and one facet in the observed WAC images are depicted.

The alignment was performed manually by shifting and rotating the visible area of shape model against each image until reaching the best match as evaluated by visual inspection. In order to estimate the uncertainty of misalignment, two best alignments were done for one particular image. The mean and standard deviation of the ratio between the two cases demonstrate the uncertainty of misalignment. This approach was repeated for the three images at different phase angles. The histograms of ratios are displayed in Figure 5.7. The symmetric shape of all histograms indicates that the misalignment uncertainty is negligible. It is noted that this alignment approach works well for most 


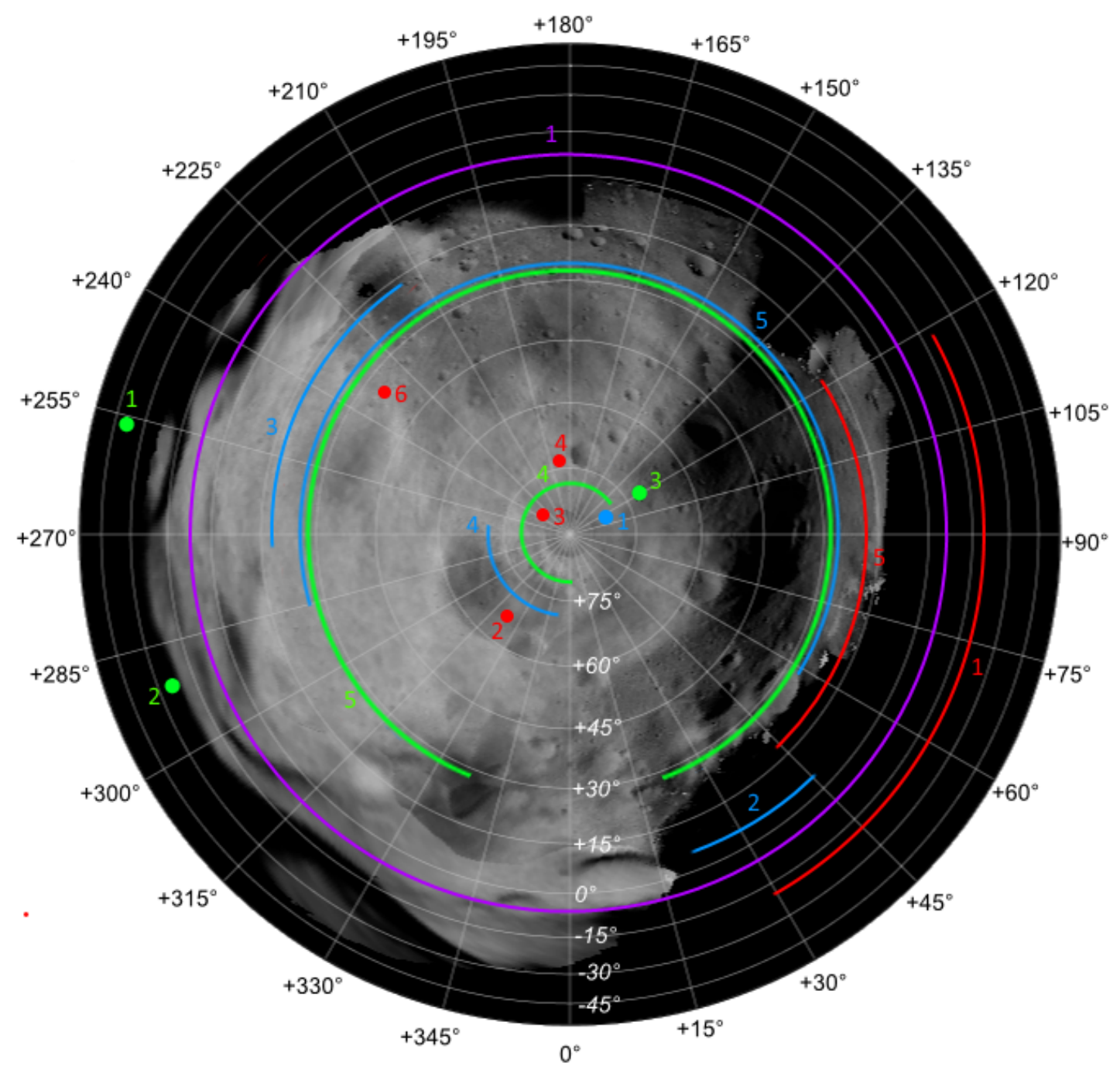

Figure 5.4: The sub-solar location of the spectra obtained by telescopic observation are plotted on the azimuthal projection map from OSIRIS images (Northern hemisphere of asteroid Lutetia). Different colors are related to the different range of the spectra (0.4-0.9 $\mu \mathrm{m}$ is green, $0.8-2.5 \mu \mathrm{m}$ is blue, 2-3.5 $\mu \mathrm{m}$ is red and 5-38 $\mu \mathrm{m}$ is violet) following Barucci et al., 2012 . 


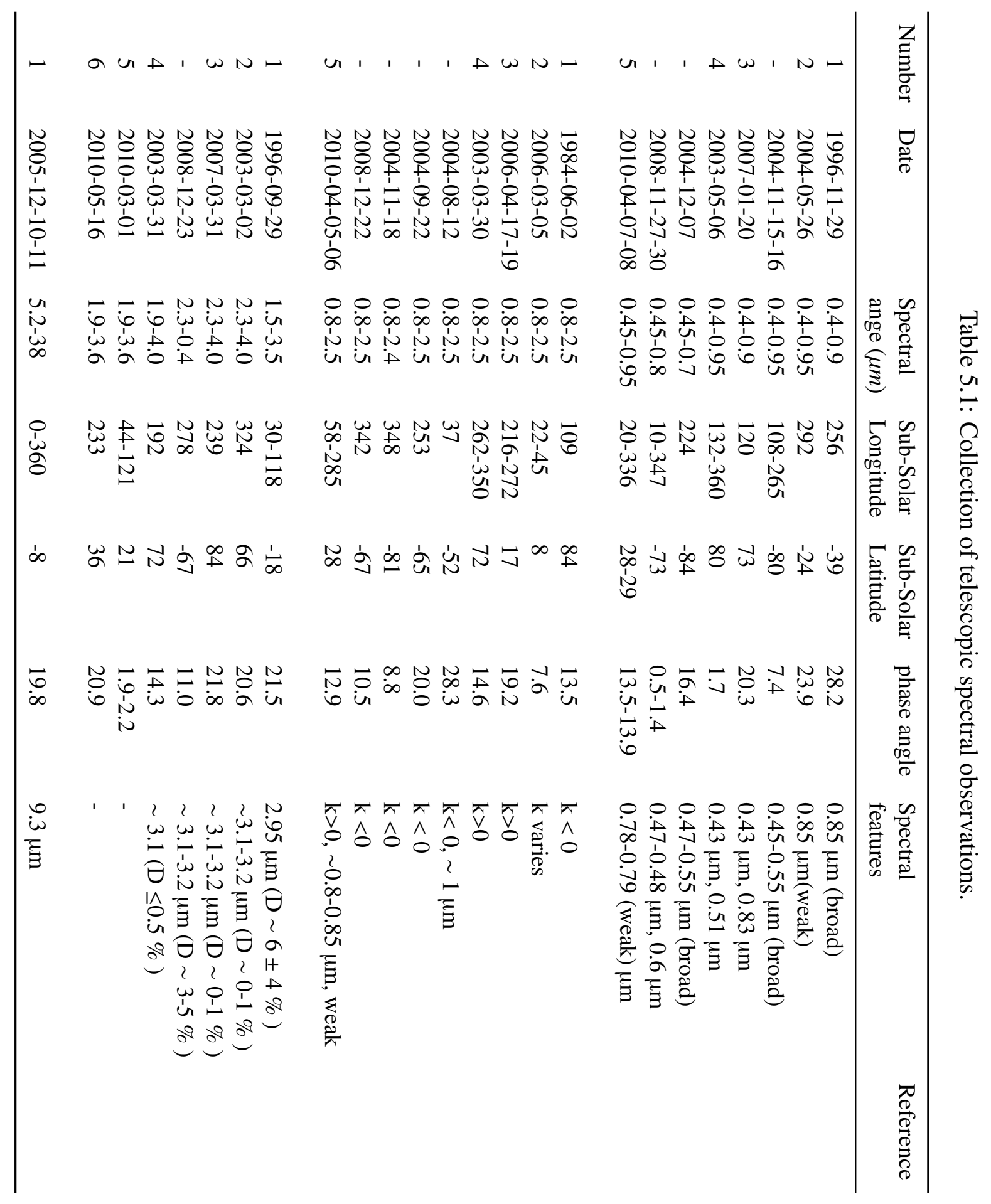




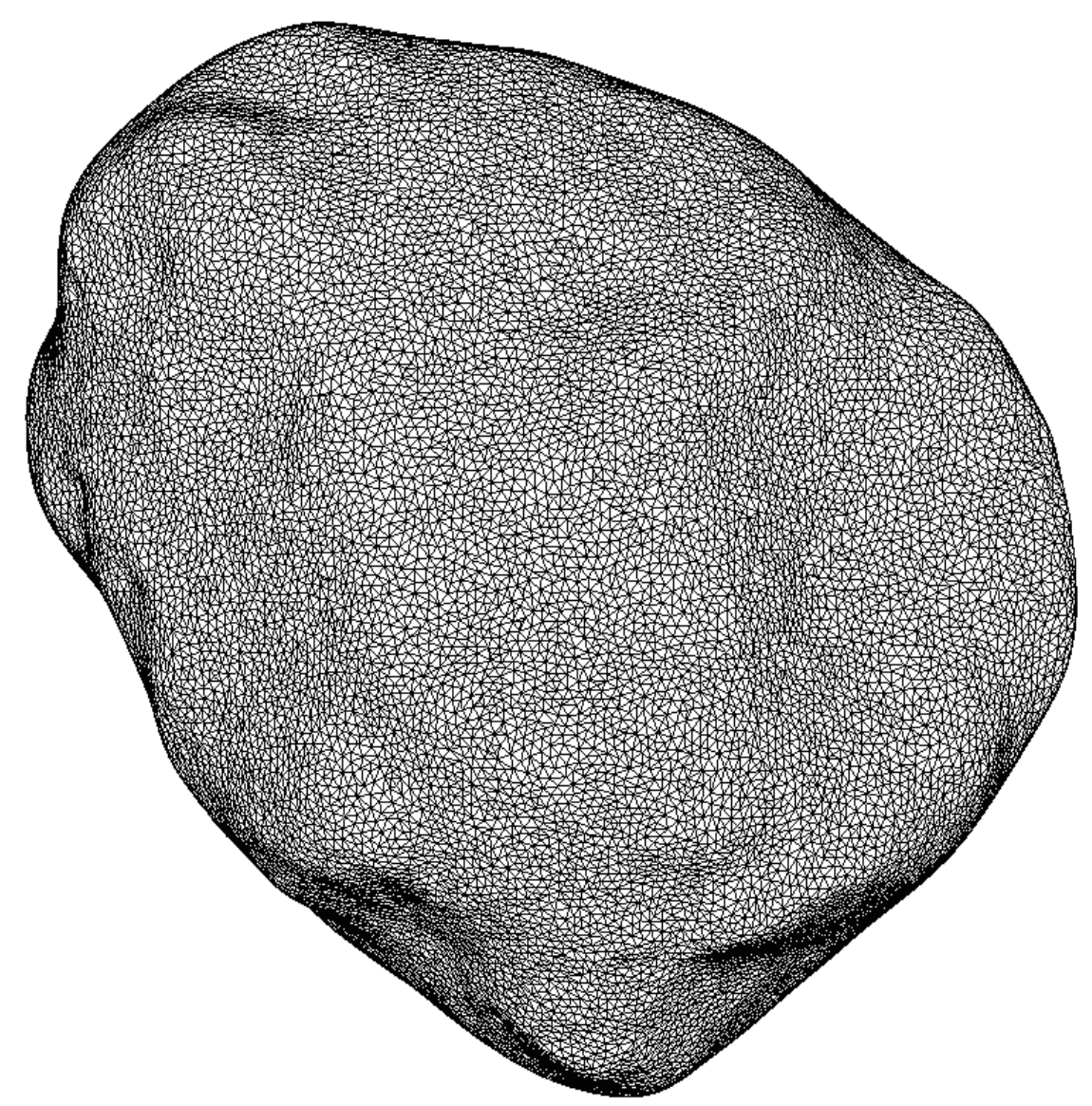

(a)

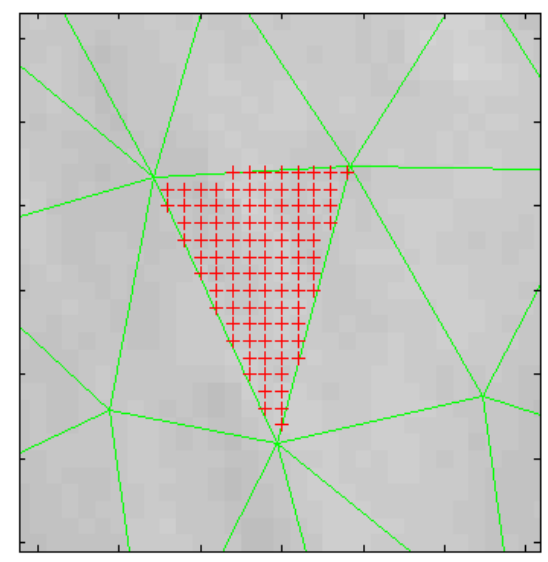

(b)

Figure 5.5: Panel (a) shows the shape model of Lutetia covered by 45778 triangular facets (Jorda et al., 2010). Panel (b) shows the example of facet projected on the NAC image of Lutetia. The facet depicted with green lines is approximately composed of 100 pixels. Pixels are marked by red cross symbol. 


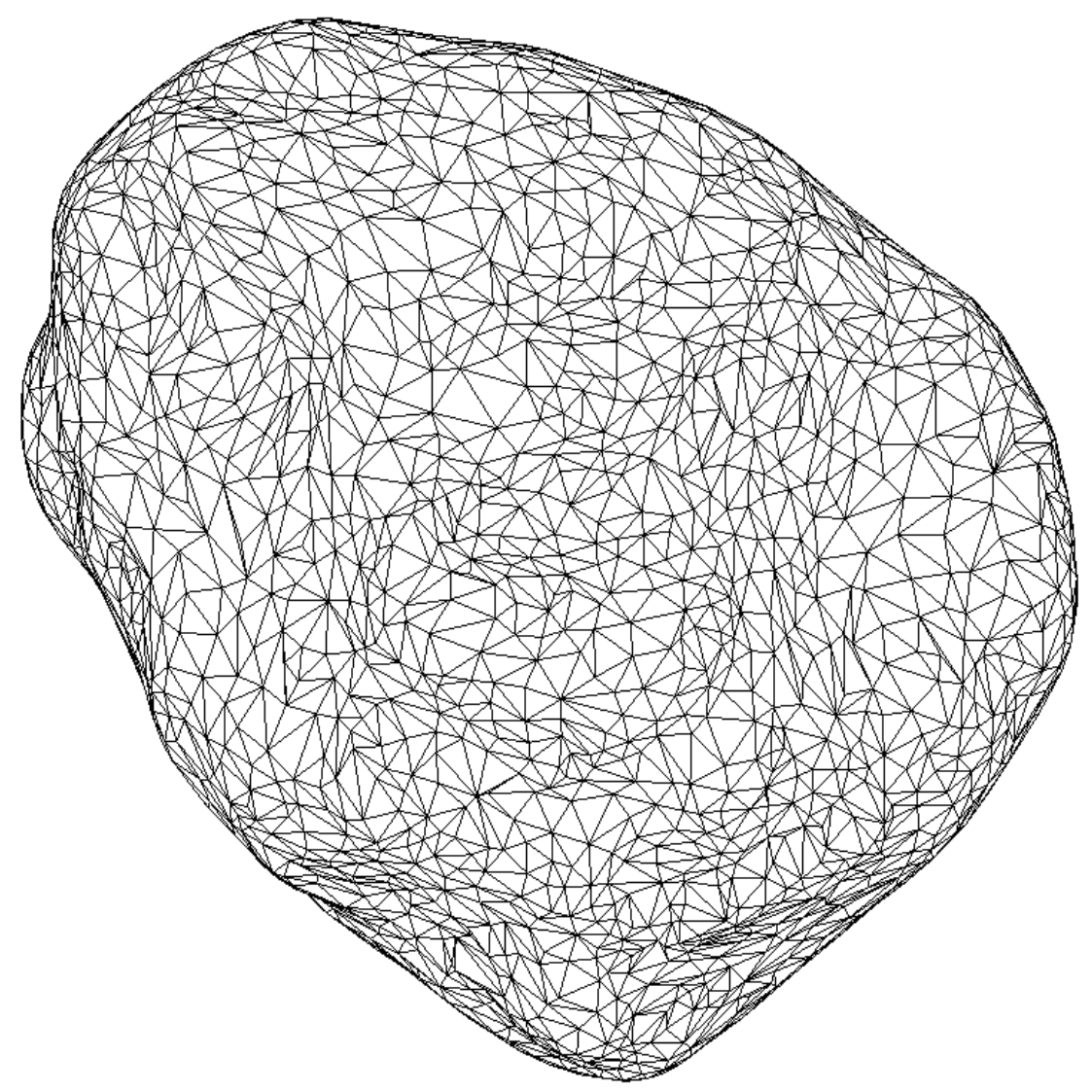

(a)

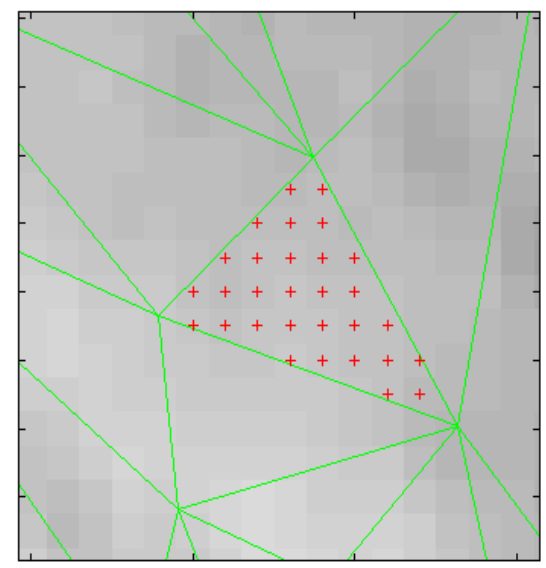

(b)

Figure 5.6: Panel (a) shows the degraded shape model of Lutetia covered by 4576 triangular facets. Panel (b) shows the example of facet projected on the WAC image of Lutetia. The facet depicted with green lines is approximately composed of 30 pixels. Pixels are marked by red cross symbol. 
part of the imaged but it is inaccurate mostly close to the limb as well as for region with shadows. Therefore, we exclude the intensity data for the high incidence and emission angles.

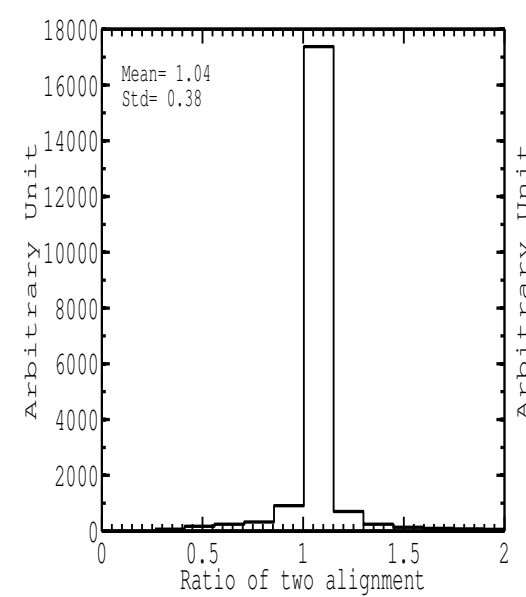

(a) Phase angle $=10^{\circ}$

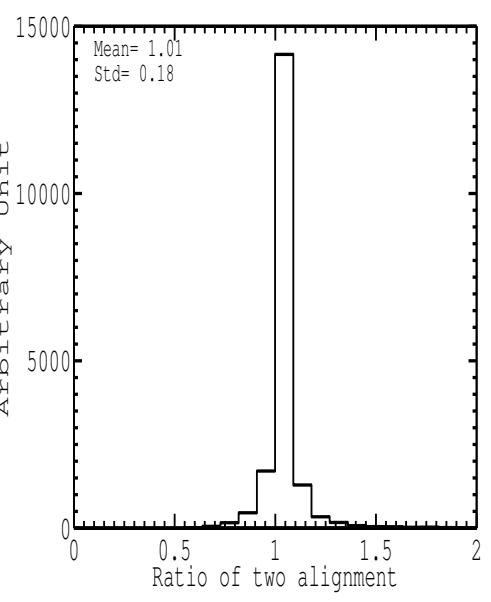

(b) Phase angle $=31^{\circ}$

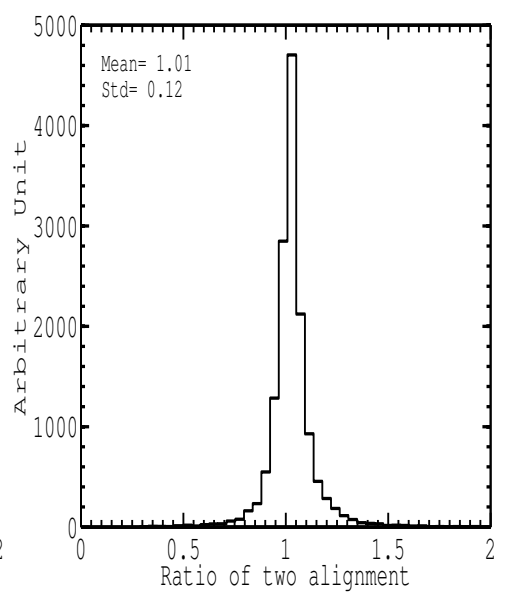

(c) Phase angle $=63^{\circ}$

Figure 5.7: The histograms of the misalignment error analysis for 3 cases.

We converted the measured intensity $\left(\mathrm{W} \mathrm{m}^{-2} \mathrm{sr}^{-1} \mathrm{~nm}^{-1}\right)$ to the radiance factor $(\mathrm{I} / \mathrm{F})$. The value of radiance factor was determined from the solar flux at the central wavelength of the corresponding filter taking into account the distance of Lutetia to the Sun $(2.72$ $\mathrm{AU})$, as described in chapter 2 .

The binning procedure is needed to perform on the data points of $\mathrm{I} / \mathrm{F}$ corresponding to the scattering angles (incidence $i$, emission angle $e$ and phase angle $\alpha$ ). The data points aggregate in $3^{\circ}$ bin group for $i$ and $e$. Binning in $\alpha$ is not done due to the limited range of phase angles covered, except for NAC F82 \& F22 images in the range between $0.15^{\circ}$ to $10^{\circ}$.

\subsection{Disk-integrated reflectance analysis}

\subsubsection{Disk-integrated phase function}

The disk-integrated reflectance of Lutetia is constructed by integrating the flux values of the visible disk of the asteroid surface above a threshold level of minimum intensity. The visible cross-section is estimated precisely, using the shape model of Lutetia.

The disk-integrated phase function of Lutetia from WAC images at $\lambda=631.6 \mathrm{~nm}$ (WAC F17) and NAC images at $\lambda=649.2 \mathrm{~nm}$ (NAC F82 \& F22) is plotted in Figure 5.8. For these filters, the available observations covered the wide range of the phase angle $\left(0.15^{\circ}<\right.$ $\alpha<156^{\circ}$ ) during the flyby, resulting in a rather complete phase function. The extensive coverage of phase angle range as well as availability of data points at nearly zero phase angle $\left(0.15^{\circ}\right)$ allow us to estimate accurately the geometric and bond albedo of Lutetia in these filters. The geometric albedo of Lutetia, $0.194 \pm 0.002$, and also the bond albedo, $0.0765 \pm 0.003$ from the disk-integrated phase function of these filters are in agreement with the albedos reported by Sierks et al. (2011). 


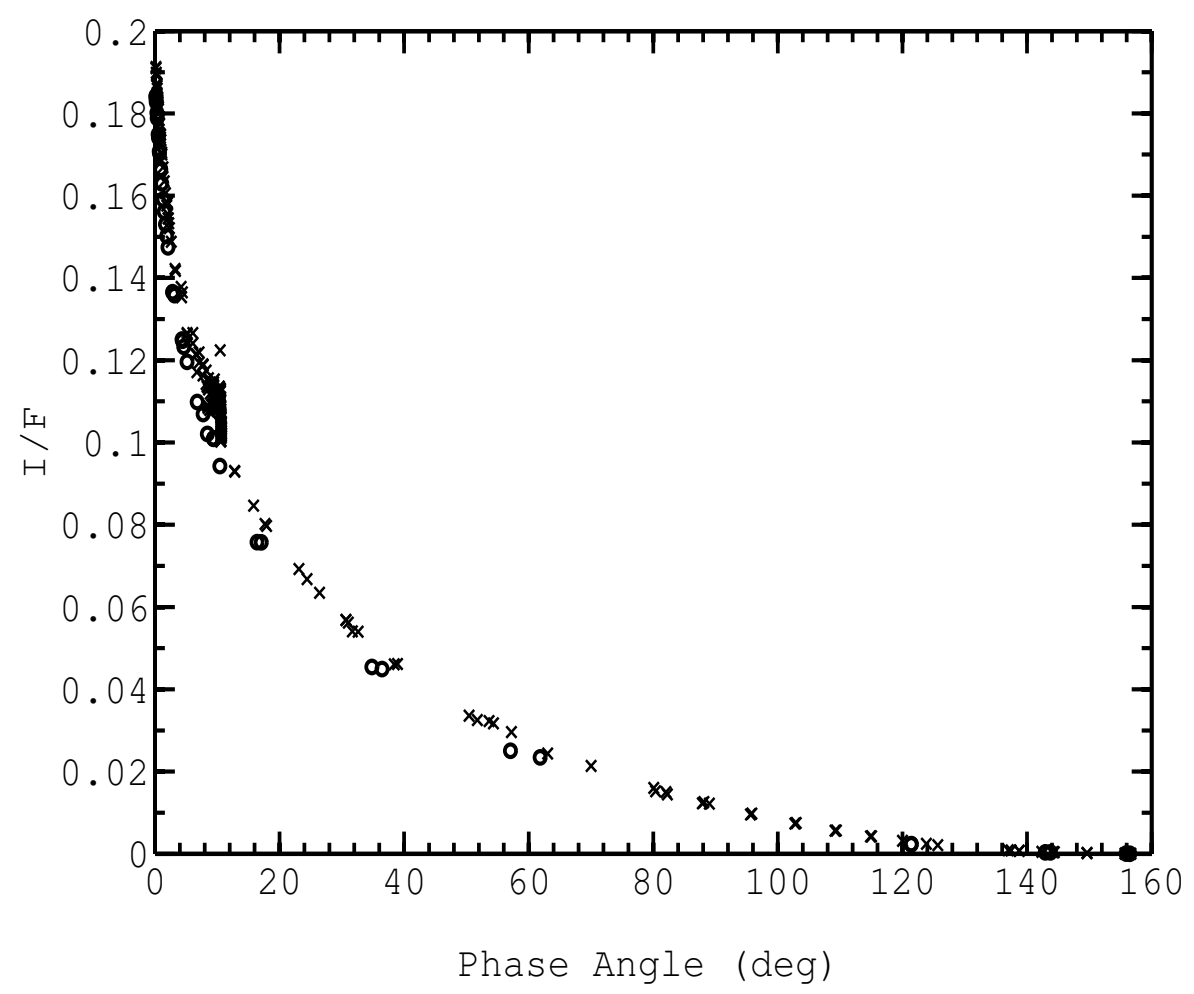

Figure 5.8: Disk-integrated phase function of Lutetia measured from OSIRIS NAC F82 $\&$ F22 images at $\lambda=649.2 \mathrm{~nm}$ and WAC F17 images at $\lambda=631.6 \mathrm{~nm}$, shown with the cross symbol. The circle symbols shows the disk-integrated phase function of Lutetia from OSIRIS NAC F16 (360.0 nm) and WAC F13 (375.6 nm). The uncertainty of the absolute radiometric calibration is reported by OSIRIS calibration team to be about $1 \%$ for all filters. The errorbar of data points are not presented in the figure because they have the same size as the symbol.

For other filters of NAC and WAC images, the disk-integrated phase function of Lutetia can be derived with the same technique. The poor coverage of phase angles for other colors do not allow us to construct the complete phase function, with the exception for the combination images of WAC F13 at $\lambda=375.6$ and NAC F16 at $\lambda=360.0 \mathrm{~nm}$ (circle symbols in Figure 5.8.

\subsubsection{Disk-integrated color}

For two different phase angles, we calculated the disk-averaged brightness of asteroid Lutetia through all filter images acquired by OSIRIS (NAC and WAC). We normalized the average $\mathrm{I} / \mathrm{F}$ of all filters to the $\mathrm{I} / \mathrm{F}$ value at $649.2 \mathrm{~nm}$. Figure 5.9 displays the normalized disk-averaged $\mathrm{I} / \mathrm{F}$ as function of wavelength at phase angle $7.7^{\circ}$. The overall linear slope is $(2.9 \% \pm 0.2)$ per $100 \mathrm{~nm}$ which is compatible with the spectral slope in the range between $536 \mathrm{~nm}$ and $804 \mathrm{~nm}$, to be $3 \%$ per $100 \mathrm{~nm}$, reported by Sierks et al. (2011).

The other disk-average spectrum of Lutetia is derived from OSIRIS NAC and WAC images around phase angle $17^{\circ}$ with $5^{\circ}$ deviation for images from $701.2 \mathrm{~nm}$ to $989.2 \mathrm{~nm}$ (Figure 5.9). The overall spectral slope is calculated to be $(1.4 \pm 0.2) \%$ per $100 \mathrm{~nm}$. The 
smaller value of spectral slope for phase angle of $17^{\circ}$ cannot be interpreted as the reliable decrease in the slope because as mentioned above, some images are taken relatively in the different phase angle with $5^{\circ}$ bin which means the spectrum is affected by not precisely measured from one fixed viewing angle.

Hence, it is not possible to determine the sign of phase reddening from disk-integrated spectrum with our data. At this resolution, it is also not viable to detect any spectral feature. However, it is obvious that the spectral shape of Lutetia's disk-integrated spectra is notably flat and straight, which agrees with VIRTIS measurements (Coradini et al., 2011).

It should be noted that the first analysis of disk-integrated spectra revealed a problem of calibration in the a number of filters of WAC and NAC images. Hence, we applied the new calibration correction to 3 filters of WAC images F51 $(295.9 \mathrm{~nm})$, F71 $(325.8 \mathrm{~nm})$, and F16 $(590.7 \mathrm{~nm})$ as well as 2 filters of NAC images F16 $(360.0 \mathrm{~nm})$ and F71 (989.3 $\mathrm{nm})$ (Personal communication). It seems that the new calibration correction is not able to solve the problem either. Therefore, the peaks appeared in the disk-averaged spectra of Lutetia (Figure 5.9) are not real. These points are corresponded to the unresolved calibration issue of WAC images of Lutetia in the filters F16 at $\lambda=590.7 \mathrm{~nm}$ and F71 at $\lambda=325.8 \mathrm{~nm}$ and they are not included to calculate the spectral slope.

\subsubsection{Hapke disk-integrated modeling}

Considering that there are a number of overlaps in the wavelength, between the filters of both NAC and WAC images, the Hapke modeling is performed on the phase function built on the combination of these filters. The images are used in the disk-integrated modeling are listed in Table 5.2. First, the complete phase function of Lutetia based on the combination of WAC F17 $(631.6 \mathrm{~nm})$ and NAC F82 \& F22 $(649.2 \mathrm{~nm})$ is thoroughly examined for Hapke modeling in the following. For the image data taken between phase angle $9^{\circ}$ to

Table 5.2: Characteristics of OSIRIS WAC and NAC images used in the disk-integrated analysis of asteroid Lutetia

\begin{tabular}{cccccc}
\hline Camera & Filter Name & $\lambda_{c}(\mathrm{~nm})$ & UTC & Number of images & Phase angle (o) \\
\hline WAC & F17 & 631.6 & $06: 35-16: 01$ & 41 & $0.33-156.68$ \\
WAC & F13 & 375.6 & $13: 48-16: 00$ & 36 & $0.15-156.51$ \\
NAC & F16 & 360.0 & $13: 45-16: 00$ & 12 & $2.82-155.90$ \\
NAC & F82 & 649.2 & $06: 18-15: 40$ & 98 & $0.15-30.67$ \\
NAC & F22 & 649.2 & $15: 41-16: 00$ & 18 & $31.69-156.23$ \\
NAC & F84 & 480.7 & $13: 45-15: 38$ & 7 & $2.95-16.88$ \\
NAC & F24 & 480.7 & $15: 41-16: 00$ & 5 & $36.03-156.02$ \\
NAC & F83 & 535.7 & $13: 45-15: 38$ & 7 & $3.08-17.41$ \\
NAC & F23 & 535.7 & $15: 41-16: 00$ & 4 & $37.20-156.13$ \\
NAC & F87 & 701.2 & $13: 48-15: 38$ & 7 & $3.38-18.49$ \\
NAC & F27 & 701.2 & $15: 48-16: 00$ & 3 & $126.71-156.34$ \\
NAC & F88 & 743.7 & $15: 31-15: 38$ & 7 & $3.52-19.03$ \\
NAC & F28 & 743.7 & $15: 42-16: 00$ & 5 & $39.73-156.45$ \\
NAC & F51 & 805.3 & $13: 50-16: 01$ & 9 & $4.16-156.55$ \\
NAC & F41 & 882.1 & $13: 51-16: 01$ & 12 & $3.93-156.56$ \\
NAC & F61 & 931.9 & $15: 31-16: 01$ & 10 & $4.08-156.75$ \\
NAC & F71 & 989.3 & $13: 51-16: 01$ & 12 & $4.08-156.86$ \\
\hline
\end{tabular}

$10^{\circ}$ before opposition, the flux calibration appeared to be less accurate for an unresolved reason; they are thus excluded from the fitting. 

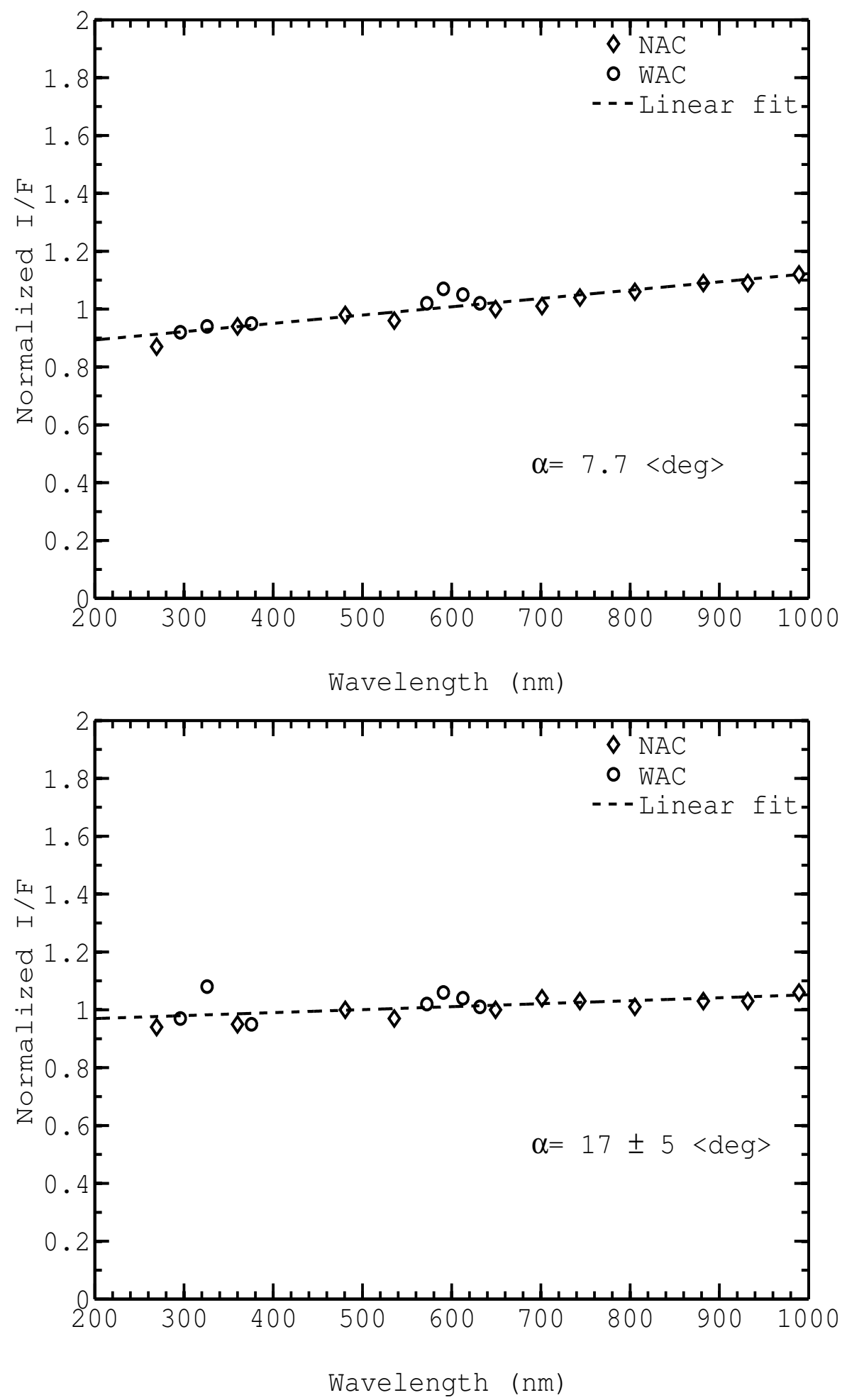

Figure 5.9: Disk-integrated spectrum of Lutetia from OSIRIS (NAC and WAC) images obtained at two phase angles normalize to I/F value of the NAC F82 \& F22 filters (649.2 $\mathrm{nm})$. The upper panel is from the images at phase angle $7.7^{\circ}$. The linear slope is $(2.9 \pm 0.2)$ $\%$ per $100 \mathrm{~nm}$ between $269.3 \mathrm{~nm}$ and $989.3 \mathrm{~nm}$. The lower panel is from the images at phase angle $17^{\circ} \pm 5^{\circ}$ with the linear slope of $(1.4 \pm 0.2) \%$ per $100 \mathrm{~nm}$ between $269.3 \mathrm{~nm}$ and $989.3 \mathrm{~nm}$. The peaks are caused due to the problem in the calibration of WAC F16 $(590.7 \mathrm{~nm})$ and WAC F71 (325.8 nm) images. 
The parameters in the Hapke equation are strongly coupled and for this reason they needed to be examined during the fitting procedure. In order to find the best-fit parameter set, several fitting tests were performed and the results are listed in Table 5.3. The first

Table 5.3: Hapke model fit to disk-integrated data of Lutetia with a single-term or doubleterm HG function for the combination of NAC F82 \& F22 (649.2 nm) and WAC F17 $(631.6 \mathrm{~nm})$ (Masoumzadeh et al., 2015).

\begin{tabular}{ccccccccc}
\hline & $\omega$ & $\mathrm{B}_{0}$ & $\mathrm{~h}$ & $\mathrm{~g}$ & $\mathrm{~b}$ & $\mathrm{c}$ & $\bar{\theta}$ & $R M S(\%)$ \\
\hline Test 1 & 0.25 & 2.48 & 0.052 & -0.18 & - & - & {$\left[25^{\circ}\right]$} & 1.4 \\
Test 2 & 0.24 & {$[1.32]$} & {$[0.02]$} & -0.33 & - & - & {$\left[25^{\circ}\right]$} & 3.8 \\
Test 3 & 0.23 & {$[1.32]$} & 0.031 & -0.33 & - & - & {$\left[25^{\circ}\right]$} & 3.4 \\
Test 4 & 0.24 & 1.53 & {$[0.031]$} & -0.29 & - & - & {$\left[25^{\circ}\right]$} & 2.1 \\
Test 5 & 0.22 & 1.67 & 0.039 & {$[-0.29]$} & - & - & {$\left[25^{\circ}\right]$} & 2.8 \\
Test 6 & 0.23 & 1.79 & 0.041 & {$[-0.27]$} & - & - & {$\left[25^{\circ}\right]$} & 1.5 \\
Test 7 & 0.24 & 1.92 & 0.044 & {$[-0.25]$} & - & - & {$\left[25^{\circ}\right]$} & 2.6 \\
Test 8 & 0.30 & {$[1.79]$} & {$[0.041]$} & - & 0.33 & 0.095 & {$\left[25^{\circ}\right]$} & 1.1 \\
\hline
\end{tabular}

five tests are devoted to explore the behavior of opposition parameters $B_{0}$ and h, in tests 6 and 7 we arbitrarily adjusted the asymmetry factor $g$ to achieve the best-fit, physically meaningful value.

In test 1, the Hapke parameters could vary freely during fitting, except for the macroscopic roughness parameter fixed to the value of $25^{\circ}$ obtained by estimating the average of all modeled macroscopic roughness values of small bodies. The macroscopic roughness parameter kept fixed for other tests in the same way. The results of test 1 yield to a rather high value of $\mathrm{B}_{0}$, suggesting that the good coverage of data points in Lutetia's phase function particularly close to zero phase angle $\left(0.15^{\circ}\right)$ do not help to constrain the opposition surge while all parameters free to model.

The opposition amplitude greater than one can be explained by the fact that if we only take into account the shadow hiding opposition effect (SHOE) mechanism with the theoretical constraint, it has the amplitude limits between 0 and 1, but according to the theoretical modeling (Hapke, 2002) other mechanisms such as the coherent backscatter opposition effect (CBOE) might contribute to the opposition surge. The amplitude parameter of $\mathrm{CBOE}$ is also constrained to be between 0 to 1 , physically. Therefore, if we consider both $\mathrm{CBOE}$ and SHOE effects together in the disk-integrated Hapke function, it means we expected to have a value up to 2 for the total opposition surge amplitude contrary to what we have from test 1 . In order to solve this problem, we compared the disk-integrated phase function of Lutetia with the a few asteroids as evaluated with Hapke modeling in Figure 5.10. The similarity of Lutetia's disk-integrated phase function with those of average S-type asteroids (Helfenstein and Veverka, 1989) help us to start our fitting procedure.

Hence, in test 2 we fixed both $\mathrm{B}_{0}=1.32$ and $\mathrm{h}=0.02$ to the values of opposition surge parameters of typical S-type asteroid (Helfenstein and Veverka, 1989). In test 3 only $\mathrm{B}_{0}$ was fixed. In test 4 , h only was fixed to the value obtained from test 2 . In order to check the variation of opposition surge parameters, we fixed in test 5 the asymmetry factor, g. By tuning up g, the fit turns to be more precise, keeping in mind that $B_{0}$ should not overreach two. The resulting attempts are shown in tests 6 and 7. According to the RMS of the 


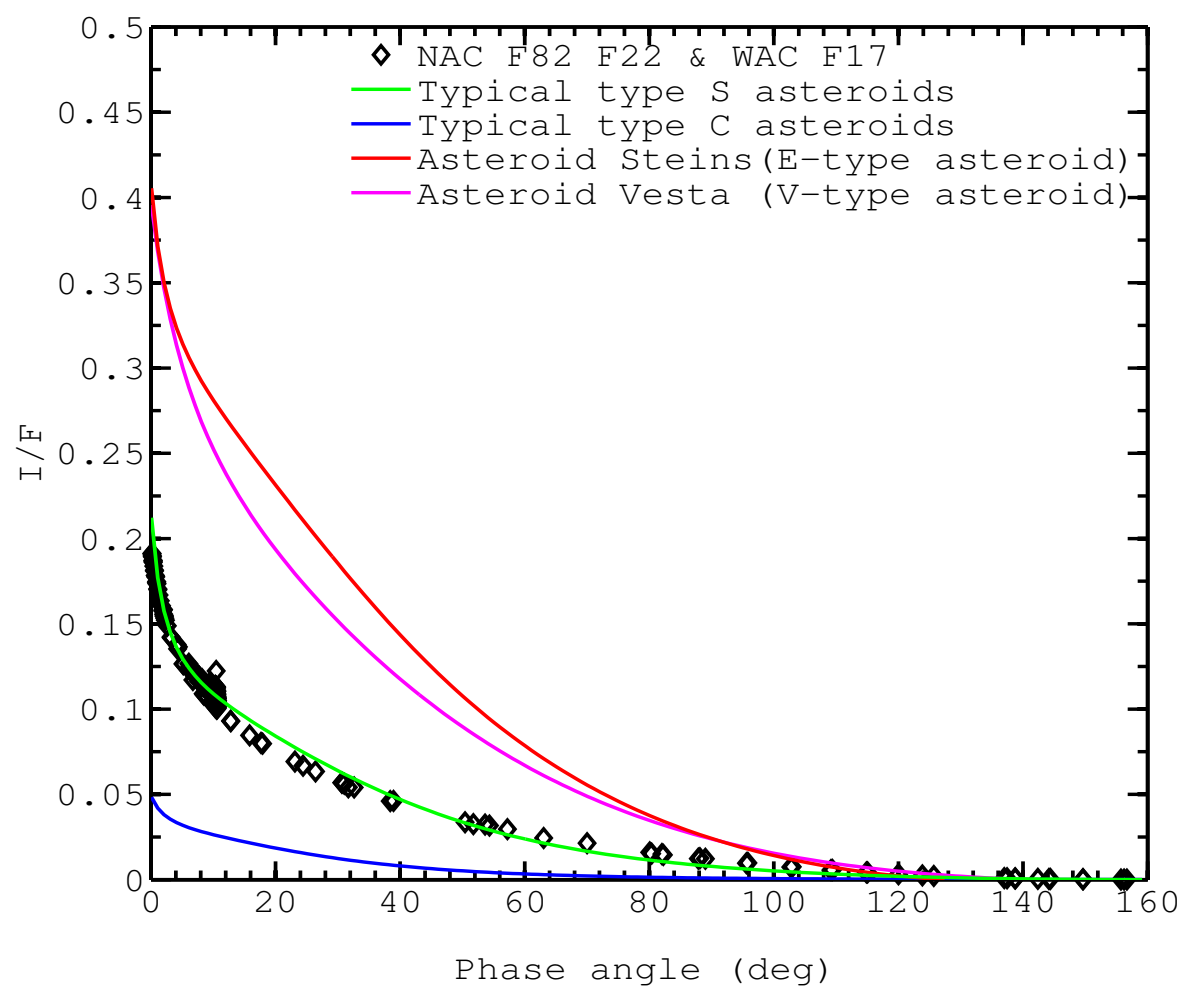

Figure 5.10: The disk-integrated phase function of Lutetia in comparison with the phase function of asteroid Steins (E-type), asteroid Vesta (V-type), the average S- and C-type asteroids based on their modeled Hapke parameters from literature (Masoumzadeh et al. 2015).

fits, the satisfactory meaningful solution for the best-fit Hapke parameters of Lutetia with a single $\mathrm{HG}$ function are $\omega=0.23, \mathrm{~B}_{0}=1.79, \mathrm{~h}=0.041, \mathrm{~g}=-0.25, \bar{\theta}=25^{\circ}$ are shown in Figure 5.11.

The wide extension of Lutetia's disk-integrated phase function allows us to include a double-term Henyey-Greenstein phase function $(2 \mathrm{HG})$ for exploring the forward scattering component. The Hapke parameters with $2 \mathrm{HG}$ function of Lutetia are estimated to be $\omega=0.30, b=0.33, c=0.095, \bar{\theta}=25^{\circ}$, fixing the opposition effect values at $\mathrm{B}_{0}=1.79$ and $h=0.041$ as constrained by the five-parameter form of Hapke disk-integrated equation (Masoumzadeh et al., 2015).

We repeat the Hapke modeling for disk-integrated data from other filters. Other suitable filters combination are obtained from the NAC F15 images $(\lambda=269.3 \mathrm{~nm})$ and NAC F16 images $(\lambda=360.0 \mathrm{~nm})$ which are combined with the WAC images at $\lambda=295.9 \mathrm{~nm}$ (WAC F51) and at $\lambda=375.6 \mathrm{~nm}$ (WAC F13); respectively. The data points of diskintegrated $\mathrm{I} / \mathrm{F}$ from other wavelengths are very few that they do not allow to sufficiently constrain Hapke parameters from disk-integrated analysis.

We decided to perform disk-integrated modeling because it is more likely to constrain the asymmetry factor, g, from our disk-integrated data compared with the available diskresolved data. According to the sensitivity analysis of Hapke parameters by Helfenstein and Veverka (1989), the particle phase function, $\mathrm{p}(\alpha)$, can be constrained by data points mostly at small and large phase angles together. We fixed $B_{0}$ to 1.79 and h to 0.041 as 


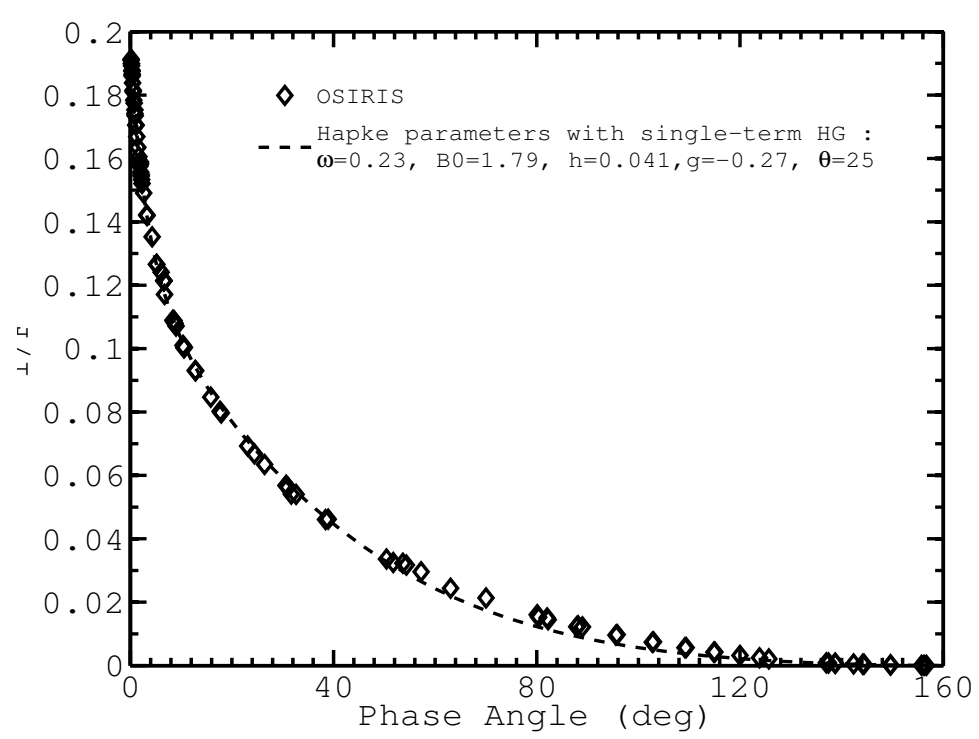

(a)

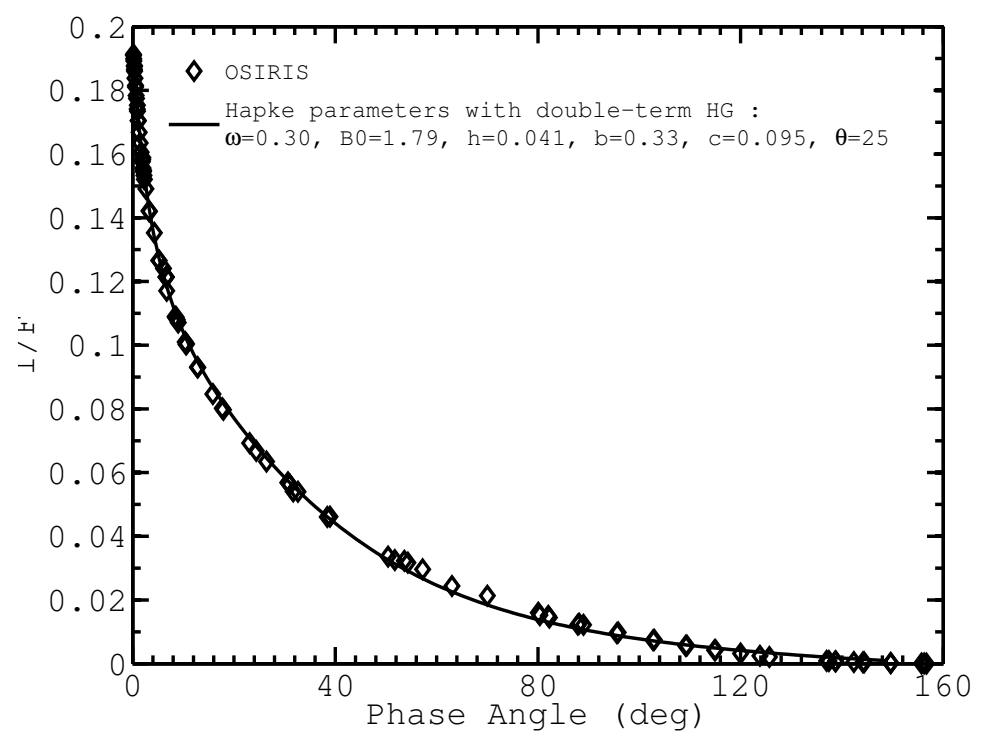

(b)

Figure 5.11: Final Hapke disk-integrated solutions with $1 \mathrm{HG}$ and $2 \mathrm{HG}$ function to diskintegrated phase function of Lutetia. Panel (a) shows the predicted phase function with $1 \mathrm{HG}$ (dash-line) corresponding to the best-fit Hapke parameter $\omega=0.23, \mathrm{~B}_{0}=1.79$ $\mathrm{h}=0.041, \mathrm{~g}=-0.27, \bar{\theta}=25^{\circ}$. Panel (b) shows the phase function (solid line) estimated from the best-fit values of Hapke parameters with $2 \mathrm{HG} \omega=0.30, \mathrm{~B}_{0}=1.79, \mathrm{~h}=0.041, \mathrm{~b}=0.33$, $\mathrm{c}=0.095, \bar{\theta}=25^{\circ}$ (Masoumzadeh et al., 2015). 
well as the macroscopic roughness parameter to $\bar{\theta}$ to $25^{\circ}$ as obtained from the previous analysis. The retrieved Hapke parameters are listed inTable 5.4

Table 5.4: The best-fit Hapke parameters modeled from disk-integrated phase function of Lutetia with $B_{0}=1.79, h=0.041$ and $\bar{\theta}=25^{\circ}$ in different filters and filter combination.

\begin{tabular}{cccccc}
\hline Camera & Filter ID & $\lambda_{c}(\mathrm{~nm})$ & $\omega$ & $g$ & $\mathrm{RMS}(\%)$ \\
\hline NAC \& WAC & F15 \& F51 & $269.3 \&$ 295.9 & 0.1918 & -0.305 & 2.8 \\
NAC \& WAC & F16 \& F13 & 360.0 \& 375.6 & 0.1980 & -0.301 & 1.2 \\
NAC & F 84 \& F 24 & 480.7 & 0.2110 & -0.297 & 2.4 \\
NAC & F 83 \& F 23 & 535.7 & 0.2225 & -0.277 & 2.4 \\
NAC & F 87 \& F 27 & 701.2 & 0.2277 & -0.286 & 0.4 \\
NAC & F 88 \& F 28 & 743.7 & 0.2515 & -0.261 & 1.7 \\
NAC & F 51 & 805.3 & 0.2103 & -0.323 & 1.9 \\
NAC & F 41 & 882.1 & 0.2724 & -0.247 & 2.3 \\
NAC & F 61 & 931.9 & 0.2675 & -0.254 & 1.8 \\
NAC & F 71 & 989.3 & 0.2968 & -0.231 & 1.2 \\
\hline
\end{tabular}

\subsection{Disk-Resolved reflectance analysis}

\subsubsection{Minnaert model}

The linear form of the Minnaert equation can be written as $\log ((\mathrm{I} / \mathrm{F}) \mu)=\mathrm{k} \log \left(\mu_{0} \mu\right)+\log$ $\mathrm{A}$ and is used to obtain A and $\mathrm{k}$ by nonlinear least-squares fitting. The goodness of fit for the Minnaert modeling is depicted in Figure 5.12. The Minnaert fitting was done using $\mathrm{I} / \mathrm{F}$ data with incidence and emission angles less than $70^{\circ}$. The good correlation between modeled I/F against measured I/F indicates that the Minnaert model is qualified to explain the photometric behavior of Lutetia's surface reasonably well. No systematic trends are seen in the scatter plots of the ratio of measured $\mathrm{I} / \mathrm{F}$ to modeled $\mathrm{I} / \mathrm{F}$ with respect to scattering angles (i,e, $\alpha$ ).

The modeled Minnaert parameters versus phase angle are plotted in Figure 5.13 and tabulated in Table 5.5. It is noticeable that Minnaert $k(\alpha)$ for the surface of Lutetia is not a linear function of the phase angle. Comparing with McEwen (1991) the trend is consistent with a surface roughness parameter around $30^{\circ}$. We applied both quadratic and cubic polynomial functions using a weighted fit in order to characterize the $\mathrm{k}$ parameter versus phase angle. There is not much difference in the fit quality, however the 2nd order polynomial fit describes the trend as whole in more desirable way. The modeled Minnaert $\mathrm{k}$ parameter at opposition is estimated from a quadratic fit to be 0.53 . This value is in the same league as the limb-darkening coefficients of other small bodies.

Li et al. (2013c) introduced an empirical function to describe the phase angle dependence of Minnaert albedo i.e.,

$$
A(\alpha)=A_{0} 10^{0.4 \beta \alpha}
$$

Where $\beta$ is the phase slope parameter (in units of mag/deg) and $A_{0}$ is known as the Minnaert albedo at opposition. The $\mathrm{A}_{0}$ has the same value as Normal albedo. The empirical 


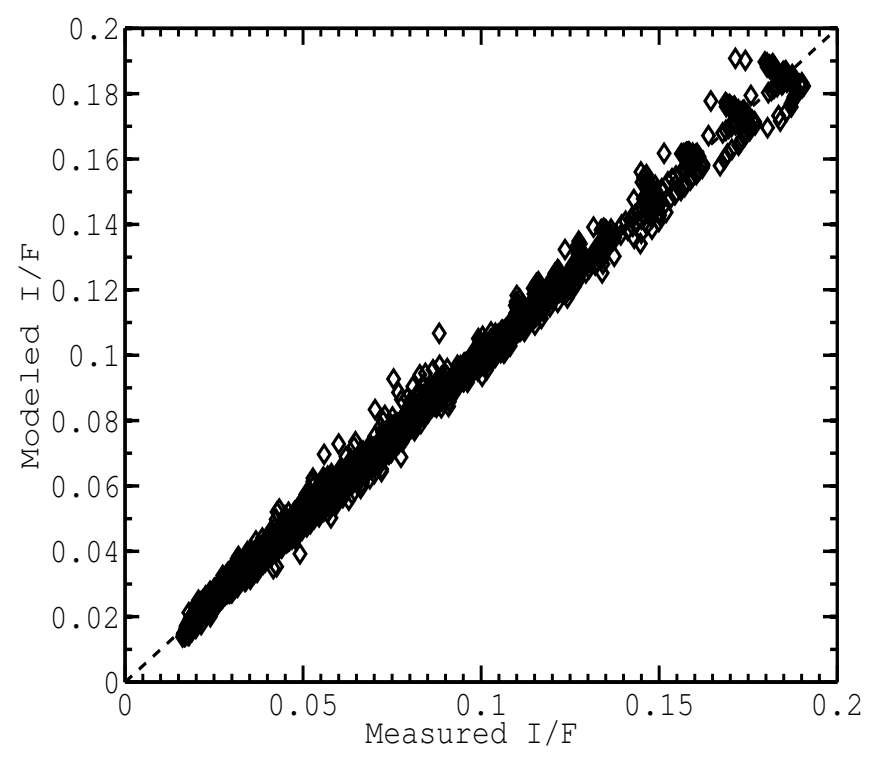

(a)

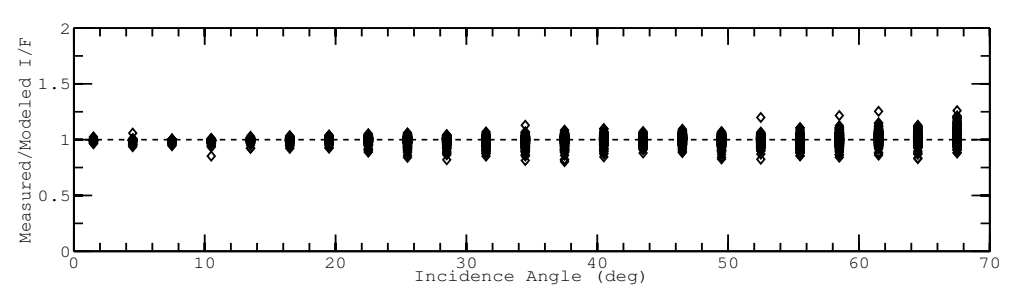

(b)

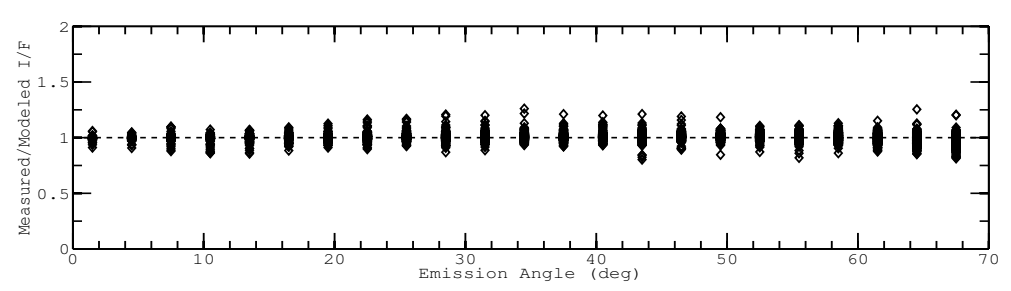

(c)

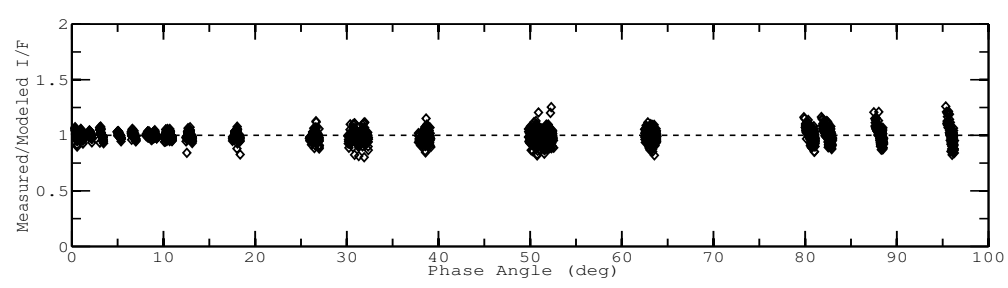

(d)

Figure 5.12: The goodness-of-fit plots for the Minnaert modeling of asteroid Lutetia from OSIRIS NAC F82 \& F22 images $(649.2 \mathrm{~nm})$. Panel (a) shows the modeled I/F against measured I/F. Panels (b), (c), (d) show the ratio between measured I/F and modeled I/F as a function of incidence angle, emission angle, and phase angle, from top to bottom, respectively (Masoumzadeh et al. 2015). 

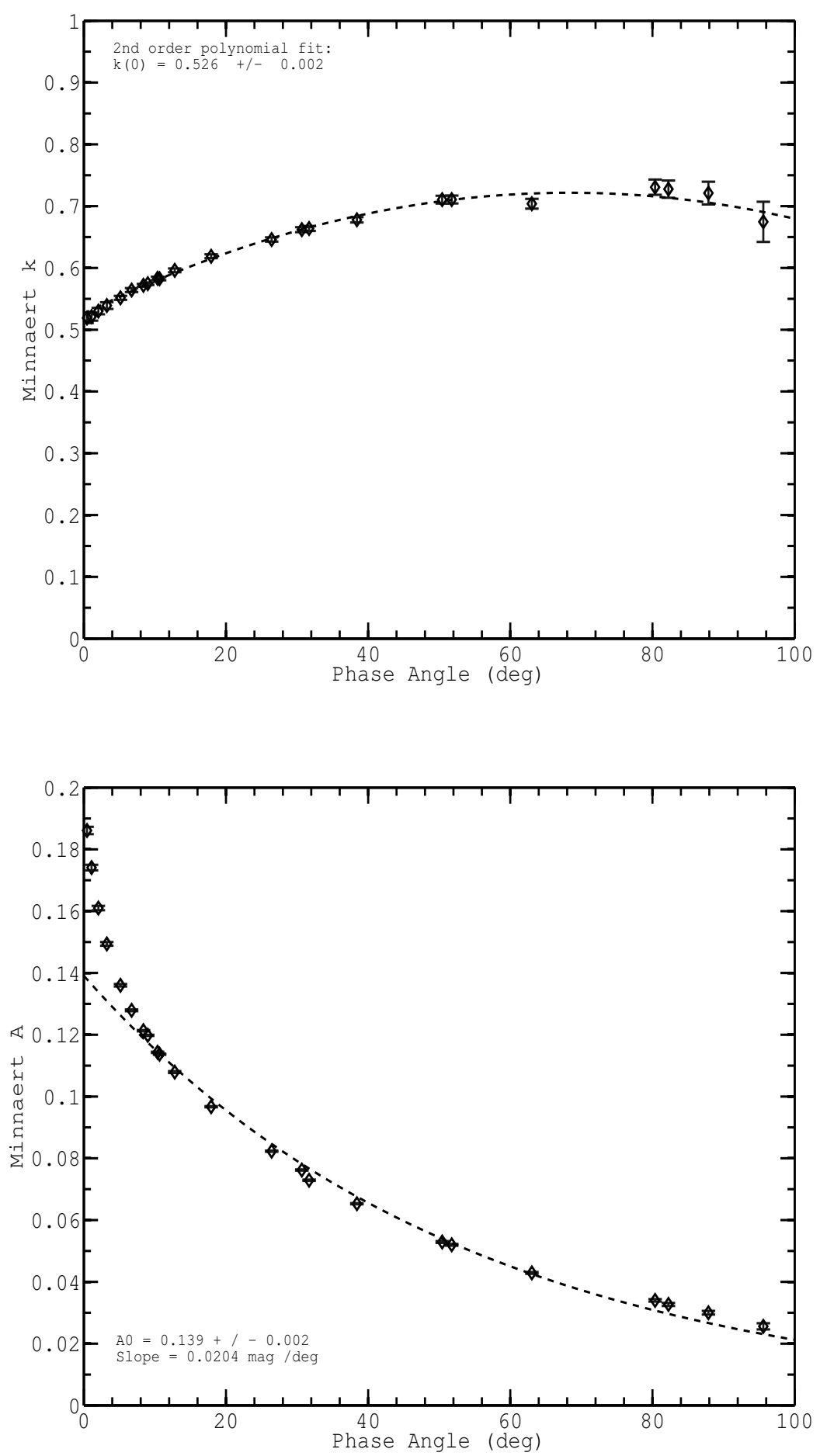

Figure 5.13: The Minnaert parameters obtained for NAC F22 \& F82 images (649.2 nm) of Lutetia. Upper panel shows the modeled $\mathrm{k}$ parameter versus phase angle. The dash line is a two order polynomial weighted fit to the $\mathrm{k}$ parameter. Lower panel displays Minnaert albedo, A, versus phase angle (Masoumzadeh et al., 2015). 
Table 5.5: Modeled Minnaert parameters for NAC F82 \& F22 images of Lutetia at $\lambda=649.2 \mathrm{~nm}$ (Masoumzadeh et al., 2015).

\begin{tabular}{lccc}
\hline Phase angle $(\mathrm{deg})$ & Minnaert k & Minnaert A & RMS $(\%)$ \\
\hline 0.5 & $0.5189 \pm 0.0082$ & $0.1861 \pm 0.00120$ & 3.5 \\
1.1 & $0.5212 \pm 0.0066$ & $0.1741 \pm 0.00090$ & 2.8 \\
2.0 & $0.5301 \pm 0.0052$ & $0.1610 \pm 0.00070$ & 2.2 \\
3.2 & $0.5391 \pm 0.0054$ & $0.1494 \pm 0.00060$ & 2.9 \\
5.2 & $0.5515 \pm 0.0032$ & $0.1360 \pm 0.00040$ & 1.7 \\
6.7 & $0.5641 \pm 0.0032$ & $0.1279 \pm 0.00030$ & 2.0 \\
8.4 & $0.5719 \pm 0.0023$ & $0.1213 \pm 0.00020$ & 1.4 \\
9.0 & $0.5744 \pm 0.0021$ & $0.1198 \pm 0.00020$ & 1.3 \\
10.4 & $0.5831 \pm 0.0027$ & $0.1143 \pm 0.00020$ & 1.8 \\
10.7 & $0.5825 \pm 0.0026$ & $0.1137 \pm 0.00020$ & 1.7 \\
12.8 & $0.5961 \pm 0.0031$ & $0.1079 \pm 0.00030$ & 2.1 \\
17.9 & $0.6189 \pm 0.0032$ & $0.0967 \pm 0.00020$ & 2.3 \\
26.4 & $0.646 \pm 0.0035$ & $0.0823 \pm 0.00020$ & 2.7 \\
30.7 & $0.6619 \pm 0.0041$ & $0.0762 \pm 0.00020$ & 3.2 \\
31.7 & $0.6638 \pm 0.0040$ & $0.0729 \pm 0.00020$ & 3.0 \\
38.4 & $0.6778 \pm 0.0043$ & $0.0653 \pm 0.00020$ & 3.2 \\
50.4 & $0.7105 \pm 0.0063$ & $0.0529 \pm 0.00030$ & 4.1 \\
51.8 & $0.7107 \pm 0.0064$ & $0.0520 \pm 0.00030$ & 4.0 \\
63.0 & $0.7038 \pm 0.0079$ & $0.0429 \pm 0.00030$ & 4.5 \\
80.4 & $0.7307 \pm 0.0124$ & $0.0340 \pm 0.00040$ & 5.2 \\
82.2 & $0.7275 \pm 0.0140$ & $0.0327 \pm 0.00050$ & 5.4 \\
87.9 & $0.7211 \pm 0.0184$ & $0.0300 \pm 0.00060$ & 6.0 \\
95.6 & $0.6746 \pm 0.0325$ & $0.0256 \pm 0.00100$ & 8.8 \\
\hline
\end{tabular}

model is applied to data in log-linear form. The fit results provide $\mathrm{A}_{0}, 0.133$ and $\beta, 0.0204$ $\mathrm{mag} / \mathrm{deg}$. Considering the fact that the value of $\mathrm{k}_{0}=0.53$ shows flatness of Lutetia's disk, the much smaller value of $A_{0}$ as representative of the normal albedo in comparison with the geometric albedo points out that $\mathrm{A}_{0}$ is not consist of opposition surge. To describe the steeper slope of the disk-integrated phase function $(\beta=0.0352 \mathrm{mag} / \mathrm{deg})$ compared with the slope of $\mathrm{A}(\alpha)$, we have to bear in mind that the Minnaert $\mathrm{A}(\alpha)$ represent the surface phase function which means it does not contain the partial illumination, whereas the diskintegrated phase function of the asteroid contains both the physical and geometric parts (Shkuratov et al., 2011).

In order to study the wavelength dependency of the Minnaert parameters, the parameters are obtained for the whole surface of Lutetia from 11 filters of OSIRIS NAC images in $5^{\circ}$ bin at $\alpha=17.9^{\circ}$. In Figure 5.14, the best-fit parameters are plotted versus wavelength. The mean value of Minnaert $\mathrm{k}, 0.62$, with the standard deviation of 0.01 confirms the lack of any variation with respect to wavelength. The trend of A spectrum is relatively flat, which is in agreement with the spectral shape of Lutetia in the visible wavelengths measured by VIRITS (Coradini et al. 2011). 

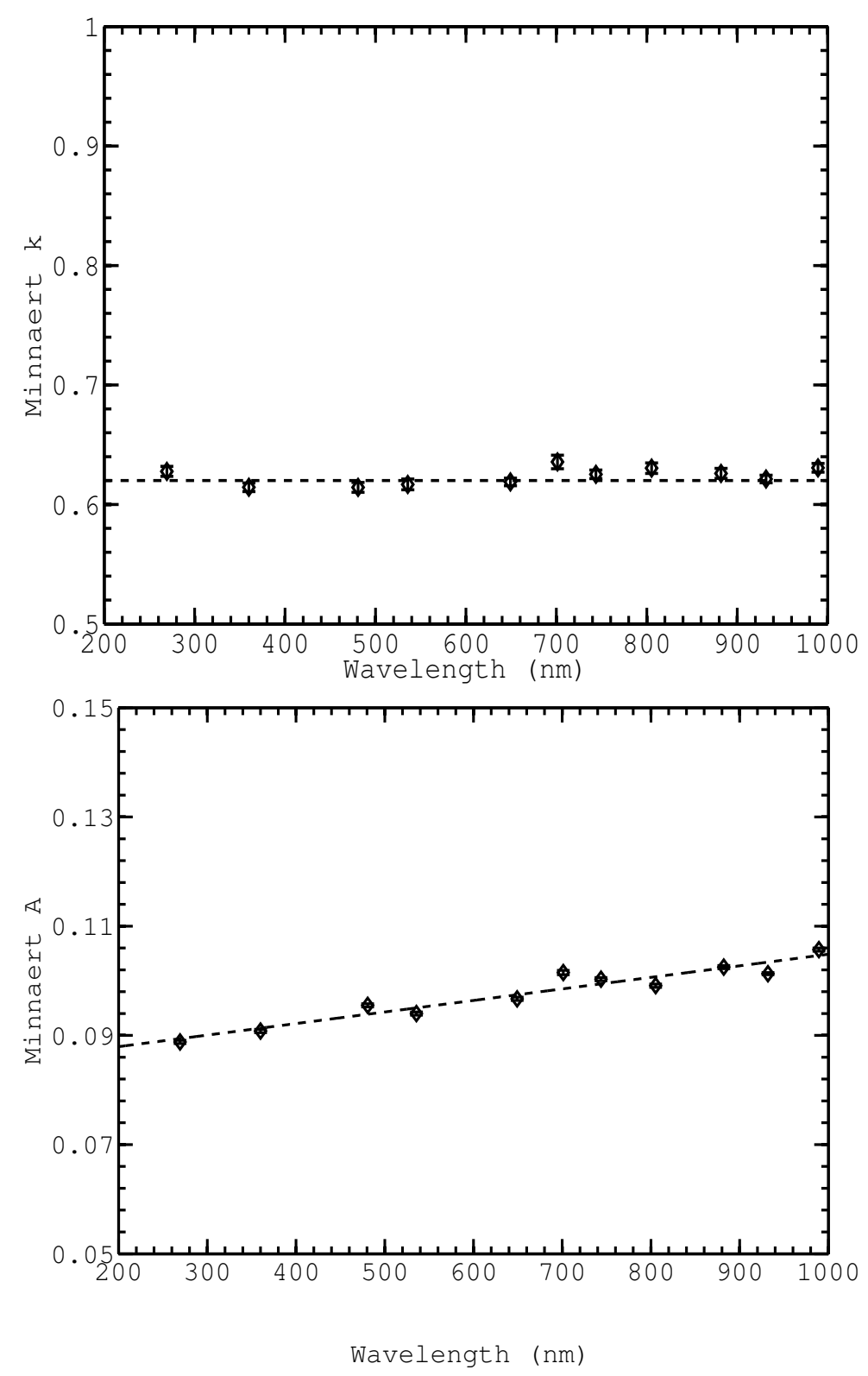

Figure 5.14: The Minnaert parameters versus wavelength from 11 filters of NAC images between the wavelength range of $269.3 \mathrm{~nm}$ to $989.3 \mathrm{~nm}$. 


\subsubsection{Hapke disk-resolved modeling}

The bidirectional reflectance model of Hapke can also be applied to the disk-resolved data. A key role of Hapke disk-resolved modeling is to derive the macroscopic roughness parameter based on characterizing the variation of intensity as a function of incidence and emission angles. In the application for the Lutetia data, a five- parameter version of Hapke's equation is used.

Similar to the disk-integrated analysis described above, we have performed the Hapke disk-resolved modeling on NAC F82 \& F22 images at $\lambda=649.2 \mathrm{~nm}$ with individual strategy due to the wide phase angle coverage. The phase angle coverage of the diskresolved images taken through other color filters is limited. For these filters, the number of images are adapted for disk-resolved modeling is not more than four images, whereas the available disk-resolved NAC F82 \& F22 images which is suitable for Hapke diskresolved modeling is 23 . The list of images used for disk-resolved analysis, are tabulated in Table 5.6

Table 5.6: Characteristics of OSIRIS WAC and NAC images used in the disk-resolved analysis of Lutetia.

\begin{tabular}{cccccc}
\hline Camera & $\begin{array}{c}\text { Filter } \\
\text { Name }\end{array}$ & $\lambda_{c}(\mathrm{~nm})$ & $\begin{array}{c}\text { Number } \\
\text { of images }\end{array}$ & $\begin{array}{c}\text { UTC } \\
\text { UTC }\end{array}$ & $\begin{array}{c}\text { Phase angle } \\
(\circ)\end{array}$ \\
\hline NAC & F82 \& F22 & 649.2 & 23 & $15: 27-15: 45$ & $0.45-95.60$ \\
WAC & F17 & 631.6 & 15 & $15: 35-15: 44$ & $8.51-70.01$ \\
NAC & F15 & 269.3 & 2 & $15: 30,15: 38$ & $2.65,15.89$ \\
NAC & F16 & 360.0 & 4 & $15: 30,15: 38,15: 41,15: 43$ & $2.82,16.39,34.82,57.08$ \\
NAC & F84 \& F24 & 480.7 & 4 & $15: 30,15: 38,15: 41,15: 43$ & $2.95,16.88,36.03,59.04$ \\
NAC & F83 \& F23 & 535.7 & 4 & $15: 30,15: 38,15: 41,15: 43$ & $3.08,17.41,37.20,60.99$ \\
NAC & F87 \& F27 & 701.2 & 2 & $15: 31,15: 38$ & $3.38,18.49$ \\
NAC & F88 \& F28 & 743.7 & 4 & $15: 31,15: 38,15: 42,15: 44$ & $3.52,19.03,39.73,65.08$ \\
NAC & F51 & 805.3 & 2 & $15: 16,15: 38$ & $4.16,19.63$ \\
NAC & F41 & 882.1 & 4 & $15: 31,15: 39,15: 42,15: 44$ & $3.93,20.19,41.09,67.15$ \\
NAC & F61 & 931.9 & 2 & $15: 31,15: 39$ & $4.08,20.80$ \\
NAC & F71 & 989.3 & 4 & $15: 31,15: 39,15: 42,15: 44$ & $4.22,21.40,42.53,69.36$ \\
\hline
\end{tabular}

Two sets of NAC F82 \& F22 images $(649.2 \mathrm{~nm})$ are used to accomplish the Hapke disk-resolved modeling. The first set involves all 23 images, covering the phase angle range of $0.45^{\circ}-95^{\circ}$. Since there was a clear systematic trend in the ratios of measured to modeled $\mathrm{I} / \mathrm{F}$ with phase angle as well as significant scattering along emission angle for four images at high phase angles $\left(80^{\circ}\right.$ to $\left.95^{\circ}\right)$, we removed data points from these images, and focused on the second set with 19 images.

All parameters are set free during the regression due to availability of the broad range of phase angles. The modeled Hapke parameters from disk-resolved analysis are listed in Table 5.7. The results are in agreement with those modeled in the disk-integrated evaluation, indicating that the Hapke parameters can be constrained reasonably well by the disk-resolved data of NAC F82 \& F22 images. We might need to verify the difference between the $\mathrm{h}=0.050$ derived from disk-resolved analysis and the $\mathrm{h}=0.041$ from diskintegrated modeling, but the discrepancy is within the 1- $\sigma$ error of the best-fit Hapke 
parameters (analyzed in section 5.5). In Figure 5.15, the goodness-of-plots indicate an acceptable fit with the linear correlation coefficient of 0.996 and the RMS residue of $3.6 \%$. No systematic trend appeared in the behavior of measured to modeled $\mathrm{I} / \mathrm{F}$ versus scattering angles $(i, e, \alpha)$. From these tests, we achieve a roughness parameter of $28^{\circ}$, the SSA of 0.226 and the asymmetry factor of -0.28 , as listed in Table 5.7.

It is potentially interesting to derive Hapke parameters from the WAC F17 images at $\lambda=631.6 \mathrm{~nm}$ using a similar analysis in order to crosscheck of the results from NAC F82 \& F22 data $(649.2 \mathrm{~nm})$. The disk-resolved I/F data of the WAC F17 images extends from $8^{\circ}$ to $70^{\circ}$. Because this phase angle range is not adequate for constraining the opposition surge parameters, we set $B_{0}$ to 1.79 and $h$ to 0.041 as modeled from the disk-integrated phase function. Agreement between the best-fit value of Hapke parameters of WAC F17 and NAC F82 \& F22 disk-resolved images are expected, except for the macroscopic roughness (Table 5.7). The two different values for macroscopic roughness are coming from the different spatial resolution of WAC and NAC images, representing the roughness at distinct size scales (Helfenstein and Shepard, 1999; Helfenstein, 1988; Shepard and Campbell, 1998).

Table 5.7: Hapke model fit to disk-resolved data of Lutetia derived from NAC images in F82 \& F22 filters $(\lambda=649.2 \mathrm{~nm})$ and WAC images in F17 filter $(\lambda=631.6 \mathrm{~nm})$. Values in square brackets were held constant during the fit (Masoumzadeh et al., 2015).

\begin{tabular}{lcccccccc}
\hline $\begin{array}{l}\text { Filter } \\
\text { id }\end{array}$ & $\begin{array}{c}\text { Pixel scale } \\
\text { range }(\mathrm{m} / \mathrm{px})\end{array}$ & $\omega$ & $B_{0}$ & $\mathrm{~h}$ & $\mathrm{~g}$ & $\bar{\theta}\left(^{\circ}\right)$ & $\mathrm{RMS}(\%)$ & $\mathrm{A}_{p}$ \\
\hline NAC & $293.65-60.33$ & 0.22 & 1.78 & 0.050 & -0.28 & 28.02 & 3.6 & 0.198 \\
WAC & $947.60-318.81$ & 0.21 & {$[1.79]$} & {$[0.041]$} & -0.29 & 24.30 & 4.7 & 0.191 \\
\hline
\end{tabular}

The Hapke disk-resolved modeling is continued for disk-resolved images of Lutetia in other filters, where the small number of images are engaged in fitting. We performed the regression with the opposition parameters fixed to $\mathrm{B}_{0}=1.79$ and $\mathrm{h}=0.041$ as well as $\mathrm{g}$ set to the best-fit value derived from disk-integrated modeling. The RMS residue are between $4 \%$ to $6 \%$, implying satisfactory fitting results (Table 5.8).

The model shows relatively high scatter for a number of filters including NACF16 $(360.0 \mathrm{~nm})$, NAC F41 (882.1 $\mathrm{nm})$ and NAC F71 (989.3). We verified the scatter plots of these cases by excluding I/F data from disk-resolved images at high phase angle. We found that these particular images cause the scatter along emission angle. Hence, we removed the $\mathrm{I} / \mathrm{F}$ data at phase angles of $57.08^{\circ}, 67.15^{\circ}$ and $69.36^{\circ}$ in NACF16, NAC F41 and NAC F71 disk-resolved data, respectively.

Further analysis is also done for NAC F51 data $(805.3 \mathrm{~nm})$ in order to understand whether the different behavior of modeled roughness parameter and modeled SSA are real or not. We changed the value of asymmetry factor, $g$, from the -0.323 derived from disk-integrated analysis to the best-fit value of -0.28 . The replacement leads to the improvement of the RMS\% residue from $4.8 \%$ to $3.9 \%$ as well as to more meaningful values for the Hapke parameters. It is also noteworthy to mention that the disk-integrated data points of NAC F51 filter is less than the other filters which may cause difficulty to find the best-fit value of $\mathrm{g}$. The other issue in this filter compared with other color filters is that the spatial resolution of disk-resolved image at phase angle, $4.16^{\circ}$ used for Hapke disk- 


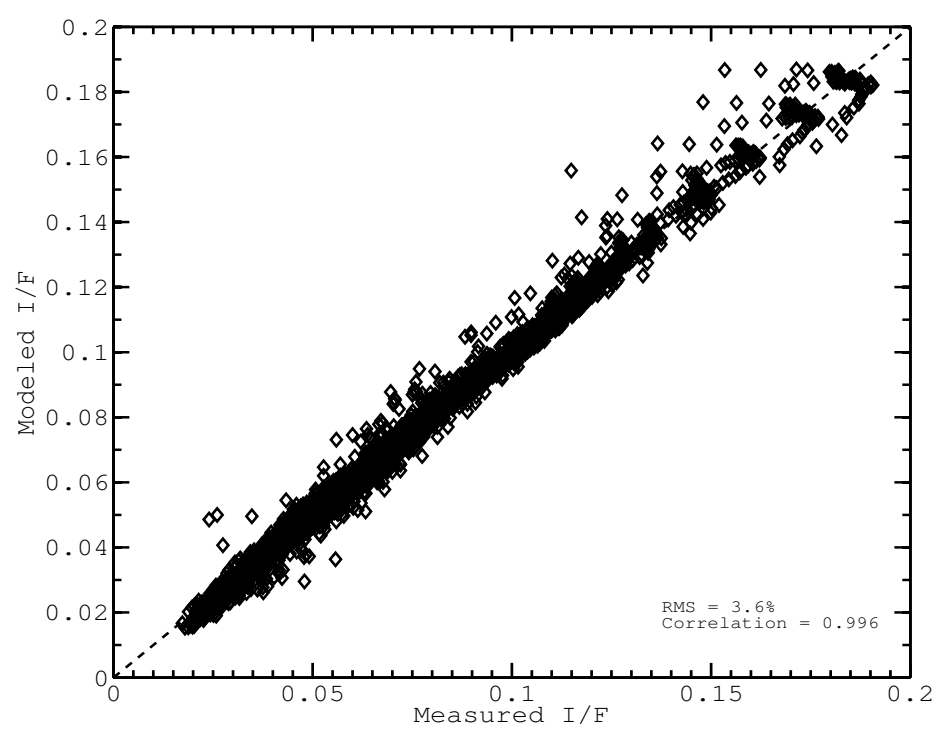

(a)

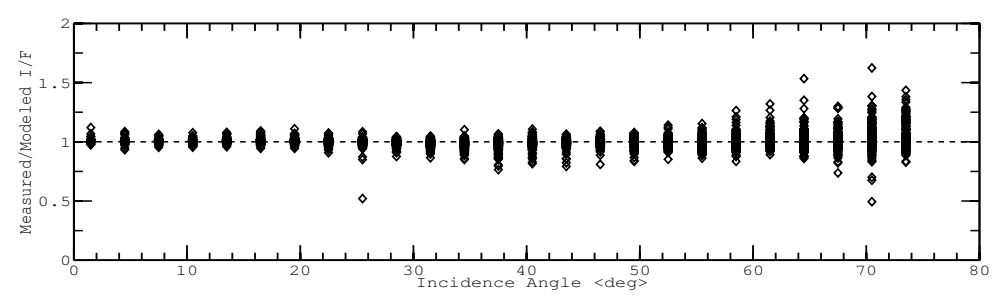

(b)

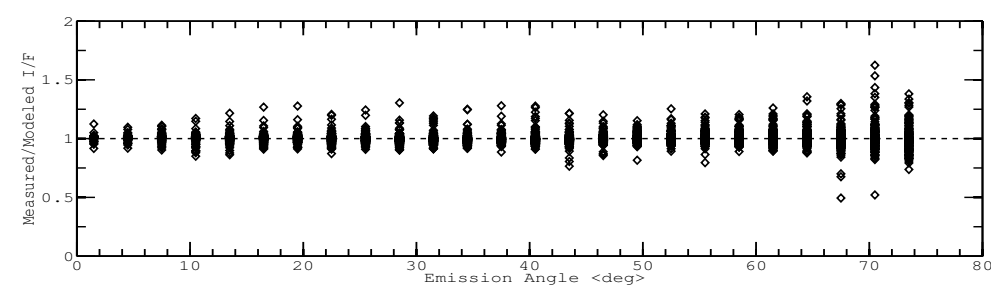

(c)

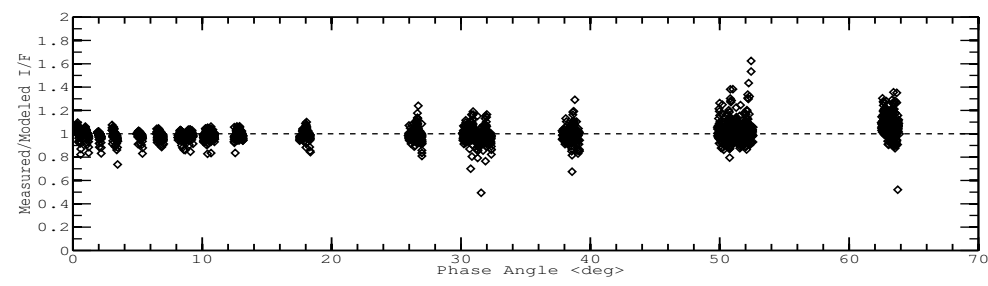

(d)

Figure 5.15: The goodness-of-fit plots for Hapke Modeling of asteroid Lutetia from OSIRIS NAC images F82 \& F22 at $\lambda=649.2 \mathrm{~nm}$. (a), (b), (c) show the ratio between measured $\mathrm{I} / \mathrm{F}$ and modeled $\mathrm{I} / \mathrm{F}$ as a function of incidence angle, emission angle, and phase angle, from top to bottom, respectively. (d) shows the modeled I/F against measured I/F (Masoumzadeh et al., 2015). 
resolved modeling is different from the images at this particular phase angle involved in the evaluation of the disk-resolved data of all filters. The final nominal Hapke solutions for the disk-resolved images of Lutetia in 10 NAC filters are tabulated in Table 5.8.

Table 5.8: The best-fit value of the Hapke parameters modeled from disk-resolved images of Lutetia for 10 NAC filters, with $B_{0}=1.79$ and $\mathrm{h}=0.041$. The $\mathrm{g}$, asymmetry factor is fixed in accordance with disk-integrated fitting values, except for NAC F51 that is fixed to -0.28 due to lack of disk-integrated data in this filter. The geometric albedo estimated from Hapke parameters according to equation 2.54 in chapter 2 and listed in table column $A_{p}$.

\begin{tabular}{cccccccc}
\hline Camera & Filter ID & $\lambda_{c}(\mathrm{~nm})$ & $\omega$ & $\mathrm{g}$ & $\bar{\theta}$ & $\mathrm{RMS}(\%)$ & Ap \\
\hline NAC & F15 & 269.3 & $0.188 \pm 0.017$ & {$[-0.305]$} & $31.4 \pm 2.0$ & 3.8 & 0.180 \\
NAC & F16 & 360.0 & $0.195 \pm 0.005$ & {$[0.301]$} & $28.5 \pm 1.4$ & 3.9 & 0.184 \\
NAC & F 84 \& F 24 & 480.7 & $0.207 \pm 0.003$ & {$[-0.297]$} & $27.5 \pm 1.2$ & 4.5 & 0.193 \\
NAC & F 83 \& F 23 & 535.7 & $0.219 \pm 0.004$ & {$[0.277]$} & $28.1 \pm 1.3$ & 4.2 & 0.190 \\
NAC & F 87 \& F 27 & 701.2 & $0.231 \pm 0.016$ & {$[-0.286]$} & $27.9 \pm 1.6$ & 5.4 & 0.207 \\
NAC & F 88 \& F 28 & 743.7 & $0.252 \pm 0.004$ & {$[-0.261]$} & $28.9 \pm 1.2$ & 4.6 & 0.208 \\
NAC & F 51 & 805.3 & $0.235 \pm 0.001$ & {$[-0.28]$} & $26.2 \pm 1.3$ & 3.9 & 0.207 \\
NAC & F 41 & 882.1 & $0.273 \pm 0.001$ & {$[-0.247]$} & $28.8 \pm 1.0$ & 4.4 & 0.216 \\
NAC & F 61 & 931.9 & $0.269 \pm 0.001$ & {$[-0.254]$} & $26.6 \pm 1.3$ & 4.9 & 0.217 \\
NAC & F 71 & 989.3 & $0.302 \pm 0.001$ & {$[-0.231]$} & $26.9 \pm 0.9$ & 4.8 & 0.227 \\
\hline
\end{tabular}

The single scattering albedo (SSA) obtained for all filters are plotted against wavelength in Figure 5.16. The modeled SSA spectrum has a slightly different spectral slope i.e. $(1.65 \pm 0.29) \%$ per $100 \mathrm{~nm}$, compared to the value of $(2.9 \pm 0.2) \%$ per $100 \mathrm{~nm}$ for the disk-integrated spectrum of Lutetia derived from the analysis in Figure 5.9). This can be explained by the fact that the disk-integrated brightness is averaged from the disk of asteroid which is coordinated in a fixed geometry, whereas the spectral variation of SSA involves all available geometry.

The $\bar{\theta}$ and the asymmetry factor, g, for all filters are plotted in Figure 5.17. It is reasonable that the roughness parameter does not vary significantly with wavelength because it is a geometric parameter that, at most, is related to the structural properties of the surface. From the values modeled from all 11 filters of NAC images, we arrive at an average value of $28^{\circ}$ for the roughness parameters with $1^{\circ}$ of standard deviation. Figure 5.17 also shows an increasing trend in asymmetry factor, $g$, of the single particle phase function with wavelength. However, we fixed the opposition effect parameters during modeling, the rather high uncertainty of $g$ value does not allow us to interpret this trend.

\subsection{Error analysis of the nominal Hapke parameters}

In this section, we used the resampling method for the estimation of Lutetia's Hapke solutions. The method was described in details for asteroid Steins in Section 4.6 in chapter 4. We resampled the I/F data points for five Hapke parameters of $\omega, \mathrm{B}_{0}, \mathrm{~h}, \mathrm{~g}$, and $\bar{\theta}$ from both disk-integrated and disk-resolved data of Lutetia. The resampling procedure based on the 


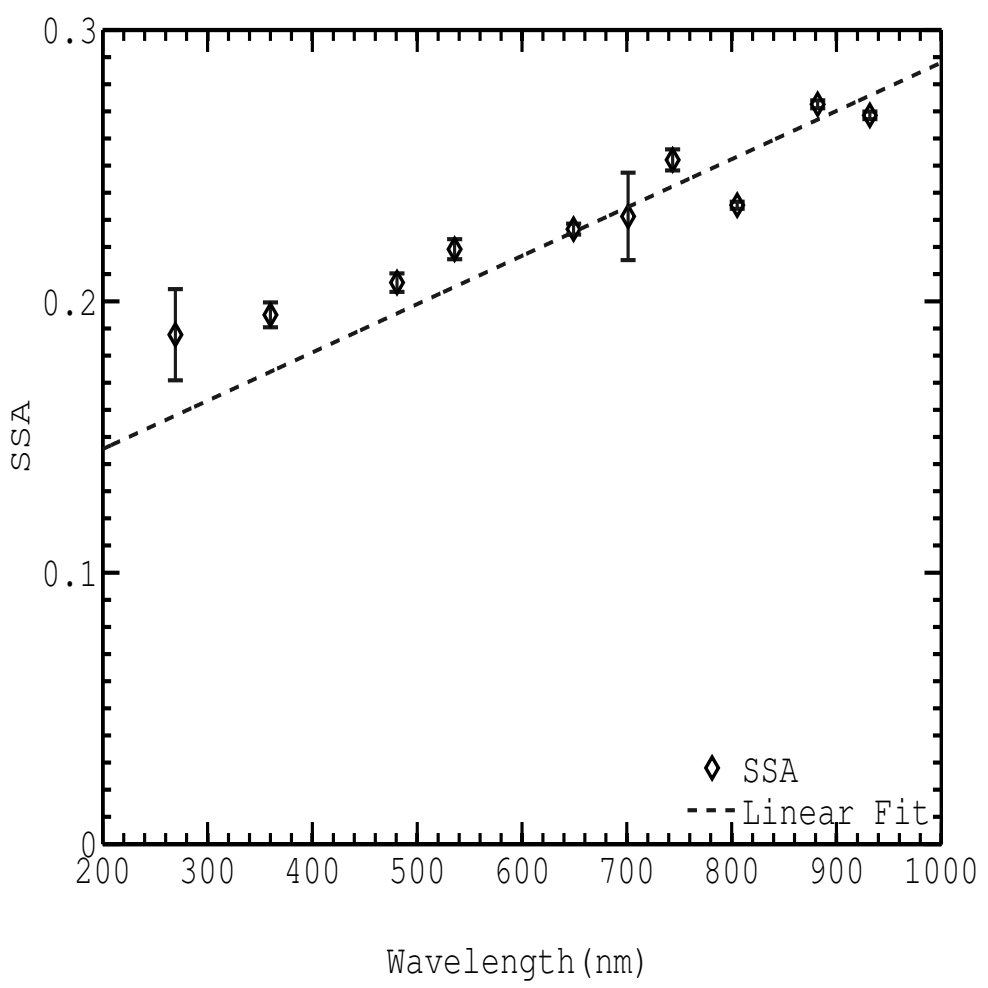

Figure 5.16: Modeled SSA of asteroid Lutetia for 11 wavelengths from OSIRIS NAC images. The linear fit indicates the trend in the spectral variation of SSA with a linear slope of $(1.65 \pm 0.29) \%$ per $100 \mathrm{~nm}$.

disk-integrated data set is performed for the combined data of NAC F82 \& F22 (649.2 $\mathrm{nm})$ and WAC F17 $(631.6 \mathrm{~nm})$ filters, while the opposition surge amplitude $\mathrm{B}_{0}$ and the macroscopic roughness $\bar{\theta}$ were fixed. From this analysis, the $1-\sigma$ error on the three Hapke parameters of $\omega, \mathrm{h}$, and $\mathrm{g}$ can be estimated to be $\pm 0.02, \pm 0.005$, and \pm 0.03 , respectively.

According to the $95 \%$ confidence interval (CI), the lower and upper boundaries of the respective Hapke model parameters are computed according to disk-integrated resampling evaluation: The $\omega$ varies between 0.195 to 0.293 , the width of opposition surge h changes from 0.034 to 0.055 . Although we fixed $B_{0}$ during bootstraping, the variation of the asymmetry factor $g$ is rather high with the range of -0.30 to -0.18 . The uncertainty of $\mathrm{B}_{0}$ is estimated while $\mathrm{g}$ and $\bar{\theta}$ are fixed. We have a standard error of \pm 0.22 for $\mathrm{B}_{0}$. The CI suggests the error bounds of $\mathrm{B}_{0}$ at 1.47 and 2.3.

The uncertainty of the Hapke parameters is also calculated by the same analysis for the NAC F82 \& F22 disk-resolved I/F data points. The uncertainty of $\bar{\theta}$ is found to be $\pm 1^{\circ}$ while none of the other Hapke parameters was fixed for the model fitting. The lower and upper boundary of $\bar{\theta}$ are $27^{\circ}$ and $29^{\circ}$, respectively. The CI of $\omega$ is between 0.216 to 0.224 , the variation of $\mathrm{B}_{0}$ and $\mathrm{h}$ are between 1.63 to 1.94 and 0.044 to 0.056 ; respectively. The CI of g-parameter is smaller in comparison with the resampling disk-integrated data from -0.29 to -0.26 . Hence, the standard errors of Hapke parameters $\omega, \mathrm{B}_{0}, \mathrm{~h}$ and $\mathrm{g}$ based on the resampling disk-resolved I/F of NAC F82 \& F22 (649.2 nm) images are as follow: $\pm 0.002, \pm 0.08, \pm 0.003$, and \pm 0.01 , respectively.

For other filters used in Hapke modeling, we also performed the resampling procedure 


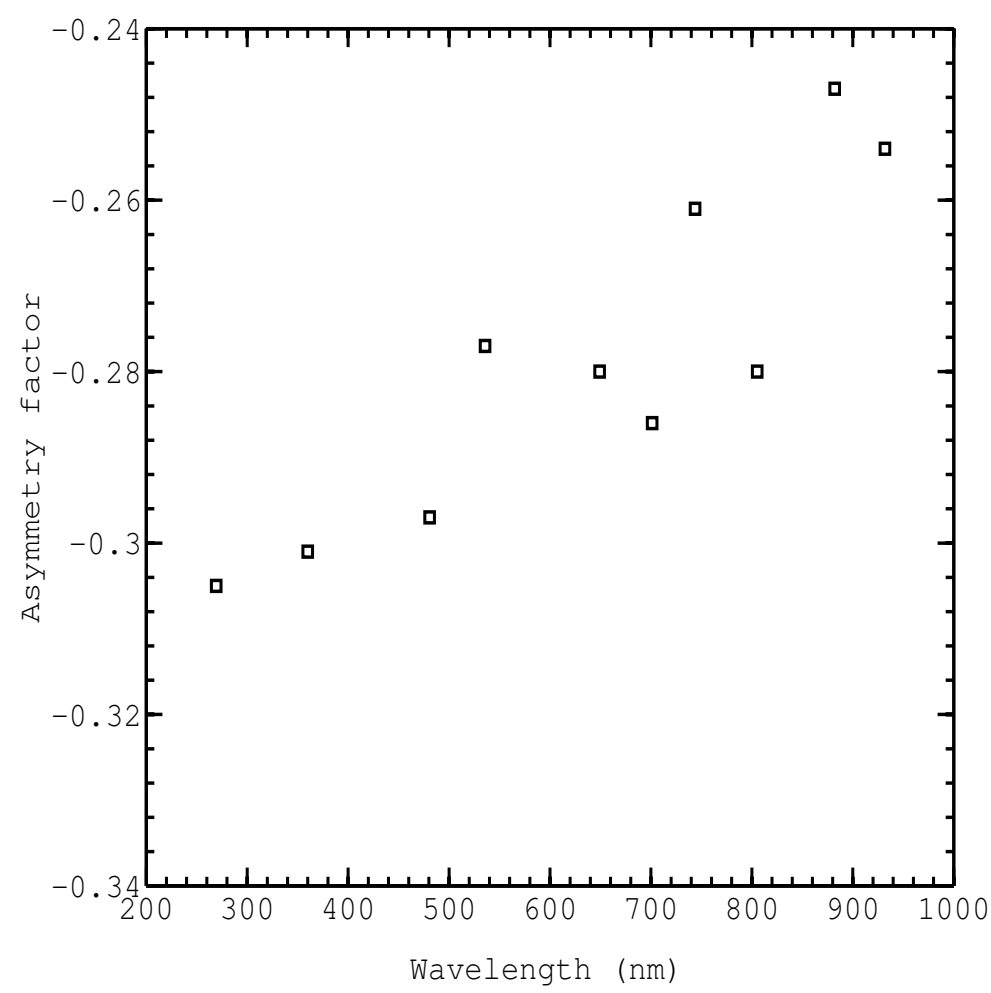

(a)

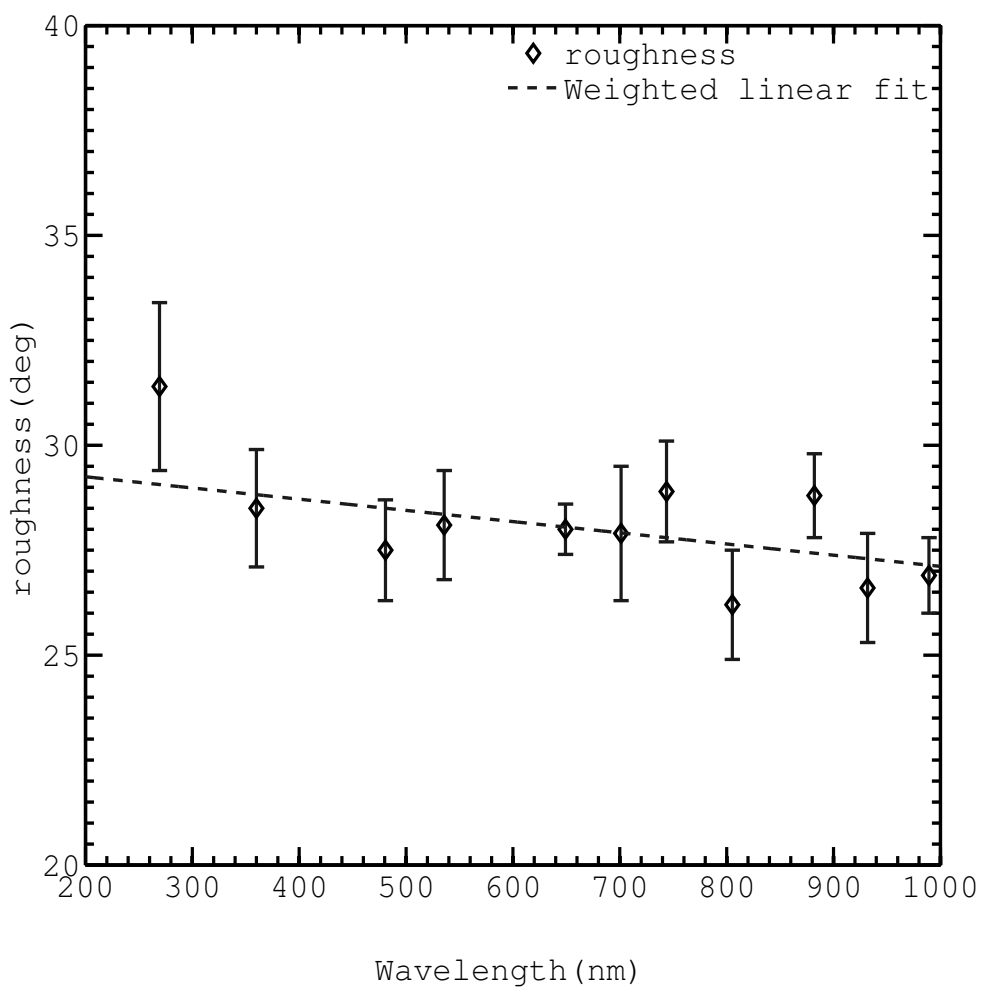

(b)

Figure 5.17: Panel (a) Variation of asymmetry factor g of Lutetia with respect to wavelength. All g-parameters are retrieved from the disk-integrated evaluation of Lutetia. Panel(b) Spectral variation of roughness parameter of asteroid Lutetia for all OSIRIS NAC filters. 
on disk-resolved and disk-integrated data. The two parameters for the opposition surge are kept fixed. The resultant uncertainty from this analysis is approximately equivalent for all wavelengths. The 1- $\sigma$ error of the roughness parameter is $1^{\circ}$, expect the NAC F15 $(269.3 \mathrm{~nm})$ and NAC F87 \& F27 images $(701.2 \mathrm{~nm})$ with a $2^{\circ}$ error. The uncertainty of SSA varies between \pm 0.001 to \pm 0.005 for all wavelengths, apart from the $1-\sigma$ error of SSA for NAC F15 $(269.3 \mathrm{~nm})$ and NAC F87 \& F27 images $(701.2 \mathrm{~nm})$ which is equal to 0.02 . The uncertainty of $\mathrm{g}$-parameter is calculated from the disk-integrated data and leads to the rather high 1- $\sigma$ errors with different values for the various filters. The estimated $1-\sigma$ errors of Hapke parameters are included with the best-fit values in Table 5.8

\subsection{Surface variation analysis}

\subsubsection{Albedo maps}

In order to assess variations of the albedo across the surface of Lutetia, one can evaluate the scattering parameters either from the Hapke modeling or the Minnaert modeling. The ratio between measured to modeled $\mathrm{I} / \mathrm{F}$ of each facet is determined. We used the modeled Minnaret parameters for the modeled I/FFigure 5.18 displays the albedo ratios mapped into the 11 images. The albedo maps show that no significant albedo variations are seen in a large-scale. The origin of the small bright contrast in the maps can be accounted for the uncertainty in the Lutetia shape model as well as to uncertainties for the co-registration approach.

Two group of images were used to analyze albedo distribution via histograms $($ Figure 5.19). The first group contains the images at small phase angles $\left(10.4^{\circ}, 10.6^{\circ}, 12.8^{\circ}\right.$, $17.9^{\circ}$, and $26.4^{\circ}$ ) where shadows do not cause significant variations. The second group presents the images with large phase angles $\left(30.7^{\circ}, 31.7^{\circ}, 38.4^{\circ}, 50.4^{\circ}, 51.7^{\circ}\right.$, and $\left.63.1^{\circ}\right)$. The FWHM of the histograms of two groups (typically $7 \%$ and $17 \%$ ) shows that the second group has a wider albedo distribution than the first one, which might be due to effects of the surface structure and/or unresolved shadows. Hence, we conclude that the variation of albedo across the surface of Lutetia is less than $10 \%$, regarding the albedo distribution of disk-resolved images between $10^{\circ}$ to $30^{\circ}$ (Masoumzadeh et al., 2015). 


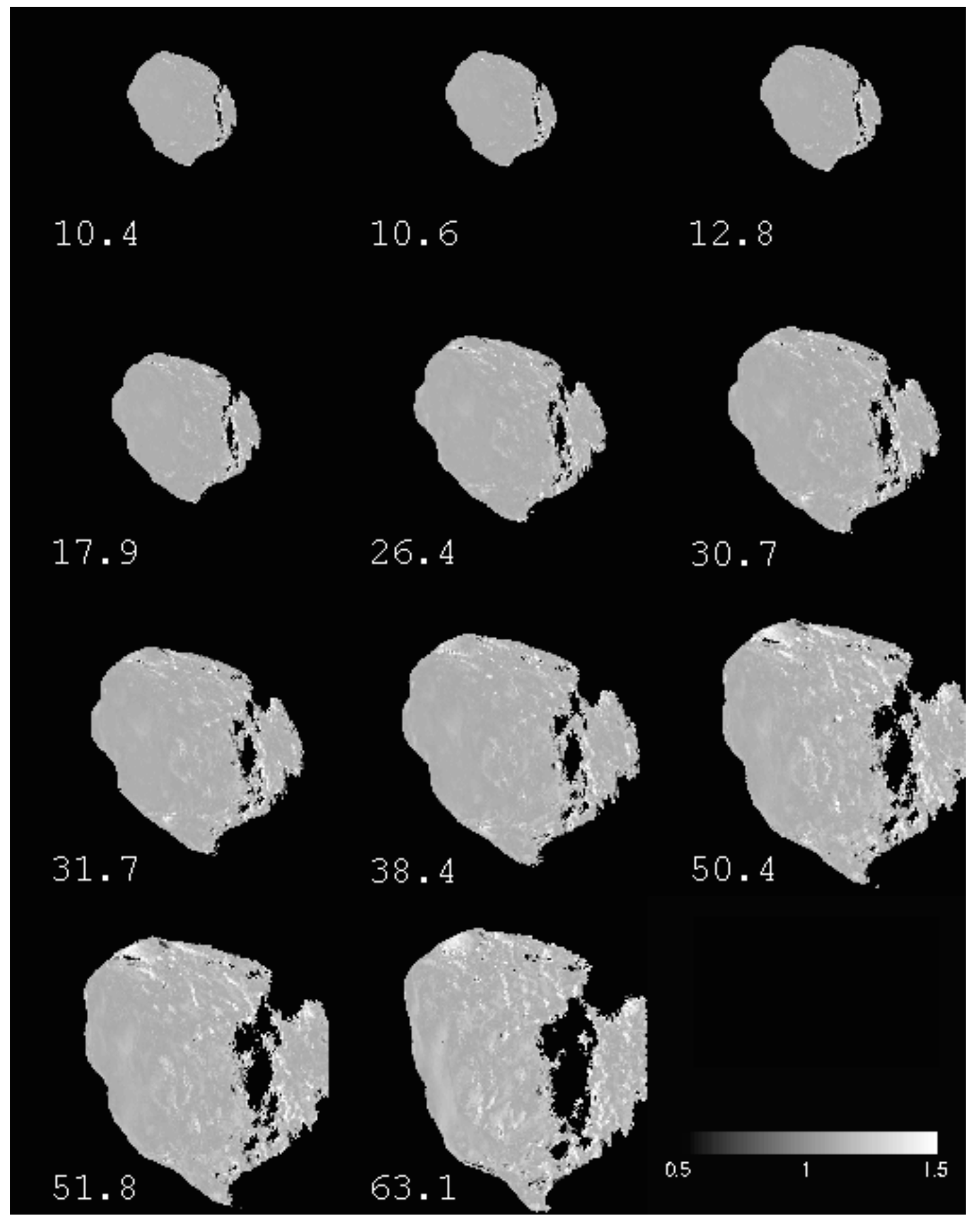

Figure 5.18: Albedo ratio maps of Lutetia from OSIRIS NAC F82 \& F22 images at $\lambda=$ $649.2 \mathrm{~nm}$, identified by their phase angles (Masoumzadeh et al., 2015). 


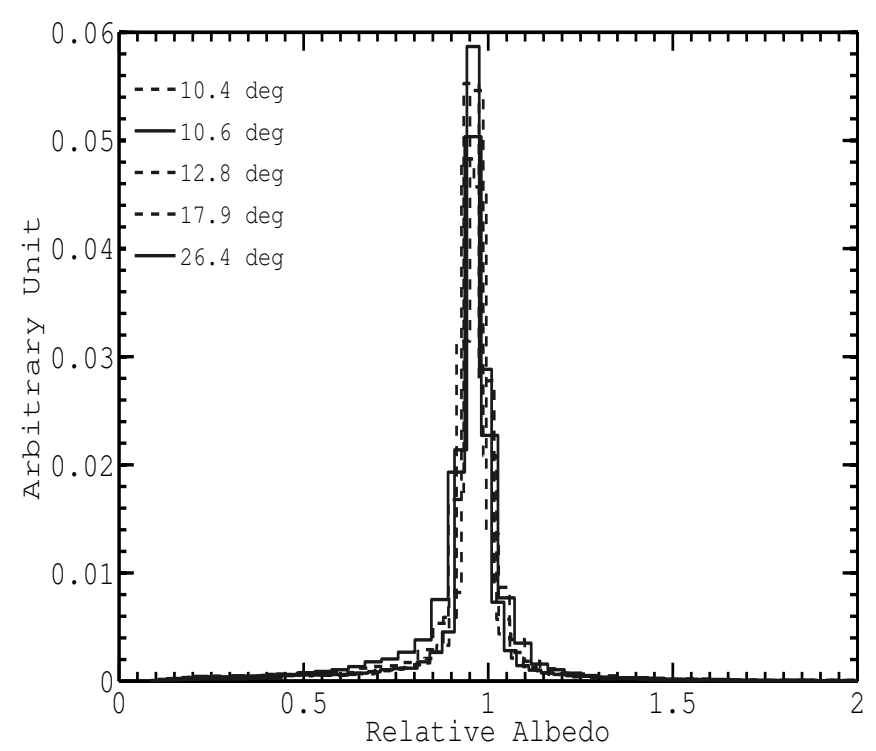

(a)

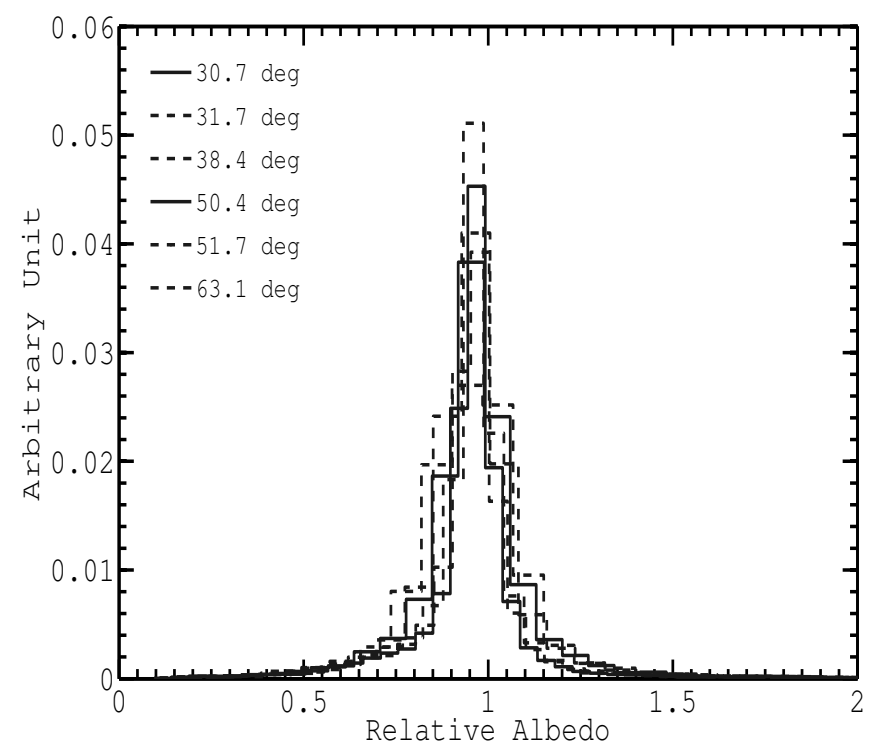

(b)

Figure 5.19: Histograms of the albedo variations on Lutetia obtained from five images at small phase angles (panel a), and six images at high phase angles (panel b) (Masoumzadeh et al., 2015). 


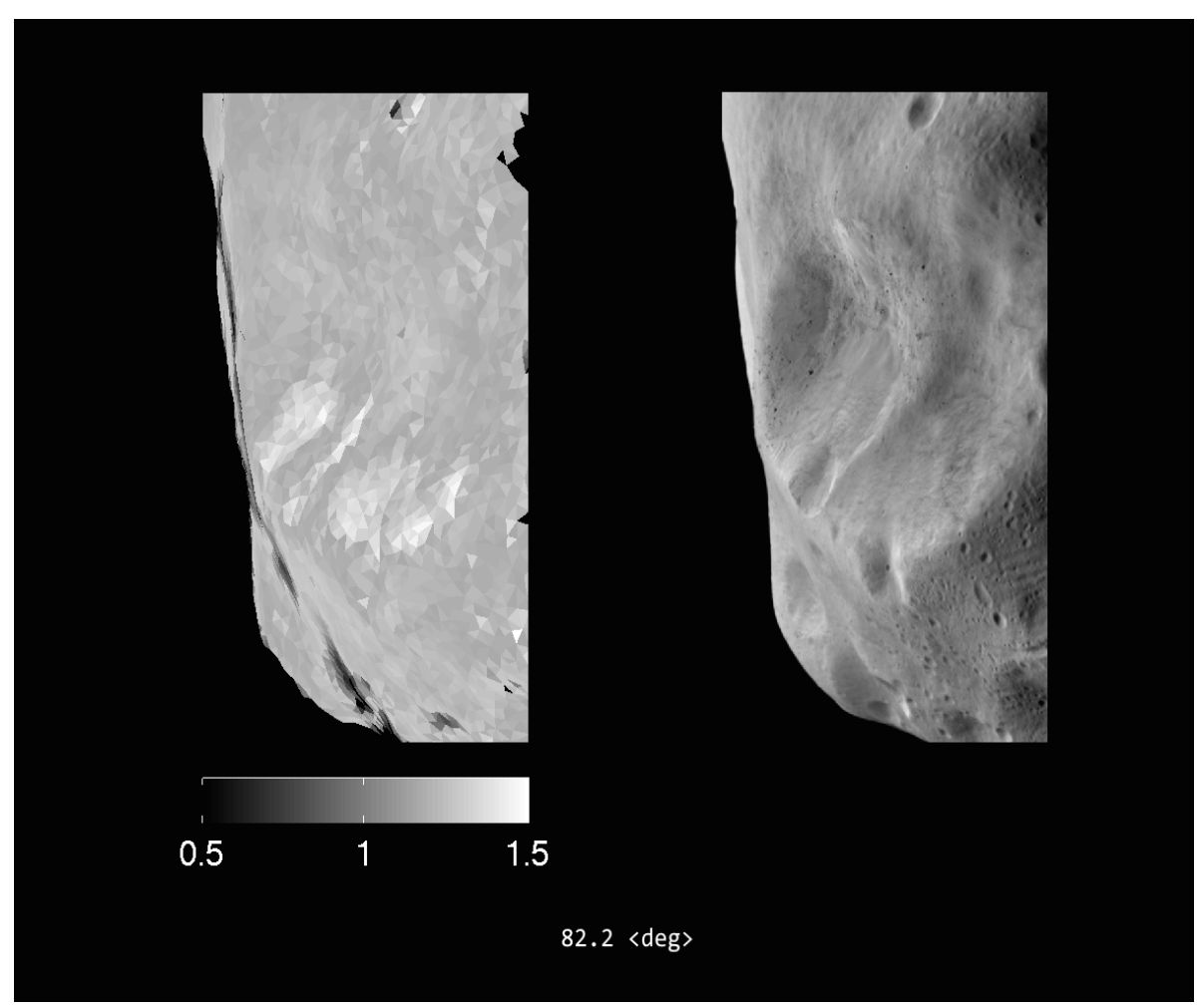

Figure 5.20: Left panel shows excerpt of the albedo ratio map of the North Polar Crater Cluster (NPCC) of Lutetia in Baetica region from OSIRIS NAC F82 \& F22 image (649.2 $\mathrm{nm}$ ) taken at phase angle $82.2^{\circ}$ at the current time of 2010-07-10T15:45:07.118. The map indicates that the measured/modeled I/F varies from $33 \%$ to $37 \%$ between the darkest and brightest regions. Right panel shows the part of the original NAC image (NAC-2010-0710T15.44.48.158Z-ID30-1251276000-F22) (Masoumzadeh et al., 2015).

At the phase angles larger than $63.1^{\circ}$, we detected by our approach the albedo variations inside the North Polar Crater Cluster (NPCC) of Lutetia as it reported by Sierks et al. (2011). Figure 5.20 displays the albedo ratio map of NPCC region, suggesting that the bright area, located on the landslide of NPCC is brighter than the dark area between $33 \%$ to $37 \%$. However, at higher phase angles, three factors should be considered that can produce the albedo contrast: (a) a resolved or/and unresolved topography may cause apparent reflectance variations across the surface. (b) the uncertainty in shape model at small scale will also introduce artifacts that can mimic albedo variations. (c) the process of photometric correction assumes that all photometric properties other than albedo remain uniform across the surface. This assumption may not be true. Thus, the higher the phase angle, the more problem this assumption may introduce in the final corrected maps. Since what Sierks et al. (2011) argued about the albedo contrast within NPCC is done for the images at phase angles of $70^{\circ}$ and $95^{\circ}$, this albedo variation would be the upper limit, and the actual variations could be smaller. For these reasons, the maps and results generated with images at low phase angles are more robust for studying albedo variations. Therefore, the $10 \%$ albedo variation based on the maps at small phase angles should be more reliable than previously reported. 


\subsubsection{Phase Ratio Maps}

The variation due to phase function and/or roughness across the entire surface of Lutetia is explored, using the results of disk-resolved reflectance modeling. The images at different viewing angles are co-registered in such way that the shape model has been aligned with the images. The alignment allows us to derive the ratio of intensity $I\left(\alpha_{1}\right) / I\left(\alpha_{2}\right)$ for each facet. The purpose of this procedure is to remove the surface albedo variations. The detection of large scale variation across the phase ratio maps should be trusted as a signature for surface property variegation while a small scale contrast can also be caused by inaccuracies in the shape model as well as uncertainties in the alignment technique.

The four phase ratio maps in Figure 5.21 illustrate an overall contrast between the 'left' and 'right' sides of Lutetia's surface. One way of exploring the possible correlation of variations to structural properties of the surface are the simulated phase ratio maps. A simulated phase ratio map can be produced by calculating the ratio of modeled intensity obtained by disk-resolved reflectance evaluations such as Minnaert or Hapke models. We used the Minnaert modeling to generate the simulated phase ratio maps.

The synthetic phase ratio maps are depicted in Figure 5.22 with the same color stretching as in Figure 5.21. It is evident from Figure 5.21 and Figure 5.22 that the simulated phase ratio maps resemble the phase ratio maps from measured data quite well. This implies that the variation identified in the measured phase ratio maps occurred as consequence of distinct scattering geometries and not for roughness and/or phase function variations (Masoumzadeh et al., 2015).

\subsubsection{Color Ratio Maps}

The main goal of color ratio maps is to intensify color difference connected to soil composition and mineralogy. The features can be distinguished as far as the contrast stretching of the color ratio images shows values deviating from the mean. The color ratio map also eliminates the effect of brightness variations caused by albedo contrast and topographic shading.

To create color ratio maps of Lutetia, four color pairs at approximately similar time and phase angle were selected from NAC images using filters F15 (269 nm), F83 (535.7 $\mathrm{nm})$, F87(701.2 nm), F51 (805.3 nm) and F61 (931.9 nm). As means to co-register images, we aligned shape model with the individual images. The ratio of $\mathrm{I} / \mathrm{F}$ is calculated for each facet shared by the pair of images.

To evaluate the continuum part of the Lutetia spectrum, two color ratios of 701.2$\mathrm{nm} / 535.7-\mathrm{nm}$ and 805.3-nm/535.7-nm were used Figure 5.23 and Figure 5.24 show the histograms of these two color ratios at visible wavelengths with the mean values calculated to be 1.07 and 1.05 in the order already mentioned. The FWHM of the color distribution in the 701.2-nm/535.7-nm and 805.3-nm/535.7-nm ratios is $6 \%$ and $8 \%$, respectively. The distribution in both ratios is not symmetric and extends moderately to the smaller color ratio values.

The data do not include incidence and emission angles greater than $75^{\circ}$ in order to remove the effect of bad co-registration which causes inaccuracies toward the limb as well as shadow regions. No significant color variations are detected in the visible part of Luteia's spectrum except for the small region near north pole of Lutetia where the cluster 


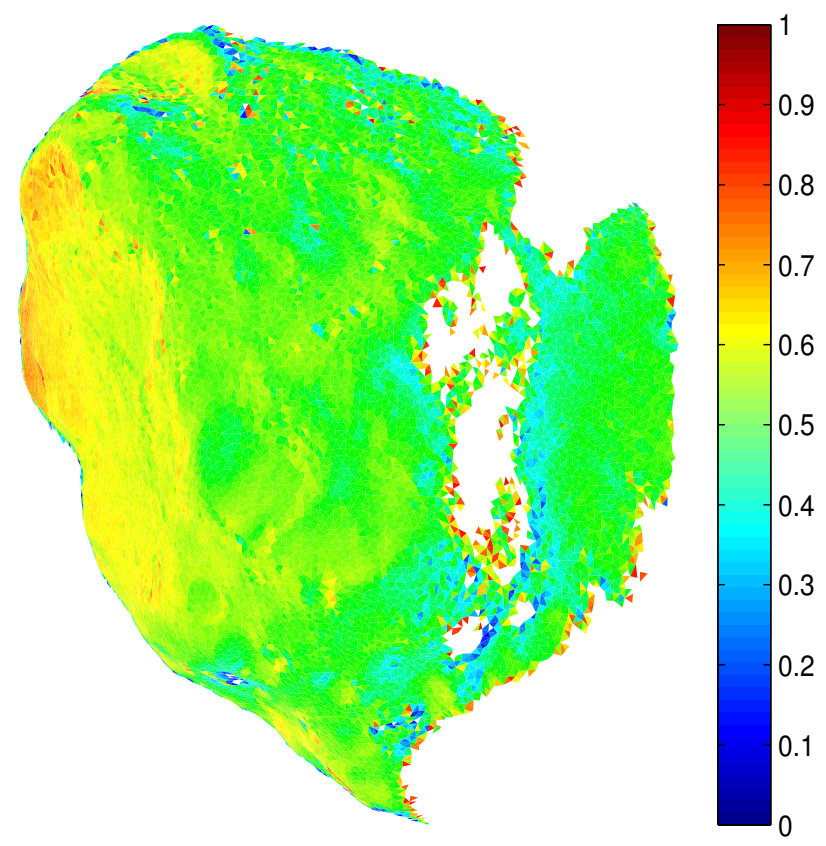

(a) $\mathrm{I}\left(50.4^{\circ}\right) / \mathrm{I}\left(31.7^{\circ}\right)$

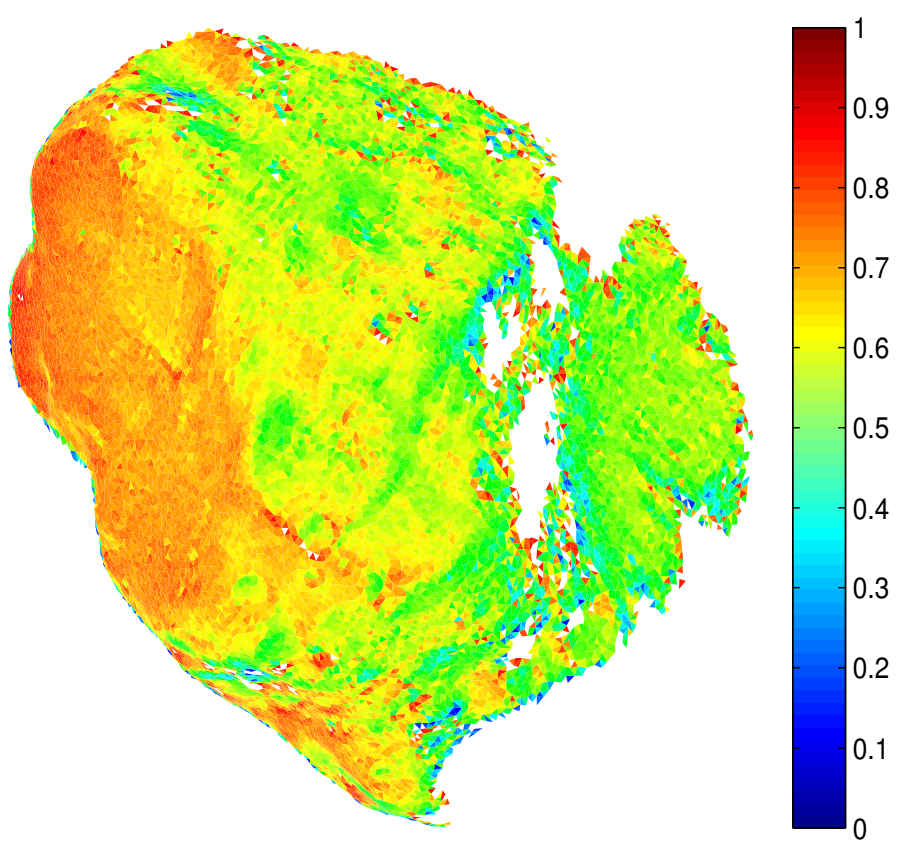

(b) $38.4^{\circ} / 26.4^{\circ}$

Figure 5.21: Phase ratio maps of Lutetia obtained from Osiris images. Panel (a) for phase angles $50.4^{\circ}$ and $31.7^{\circ}$, panel (b) for $38.4^{\circ}$ and $26.4^{\circ}$ (Masoumzadeh et al., 2015). 


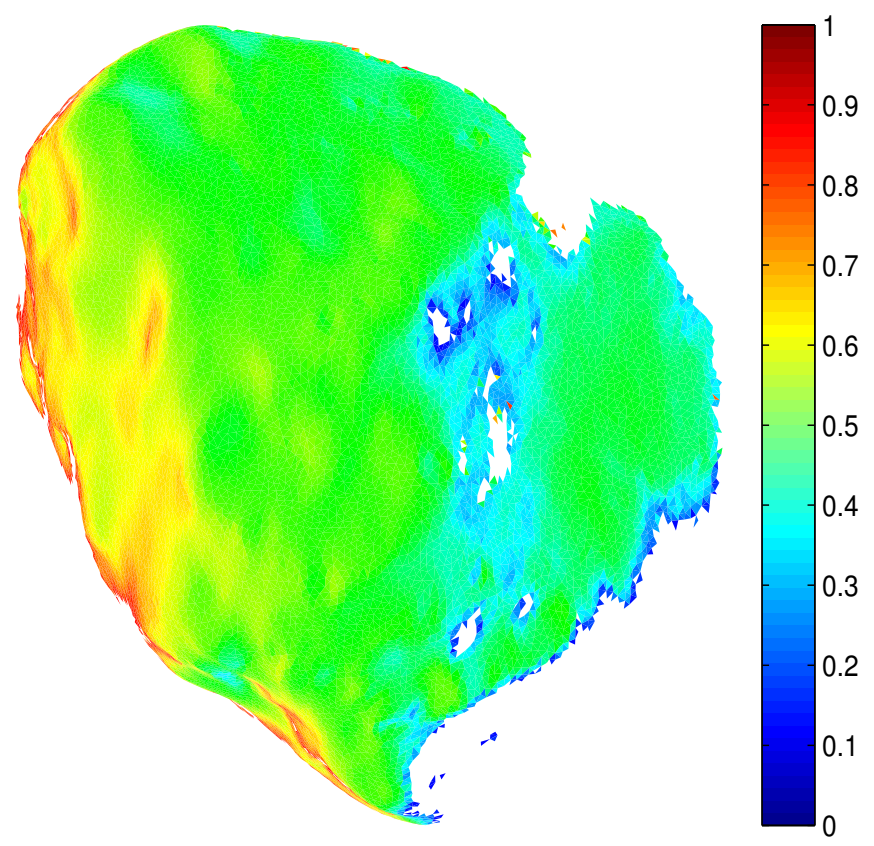

(a) $50.4^{\circ} / 31.7^{\circ}$

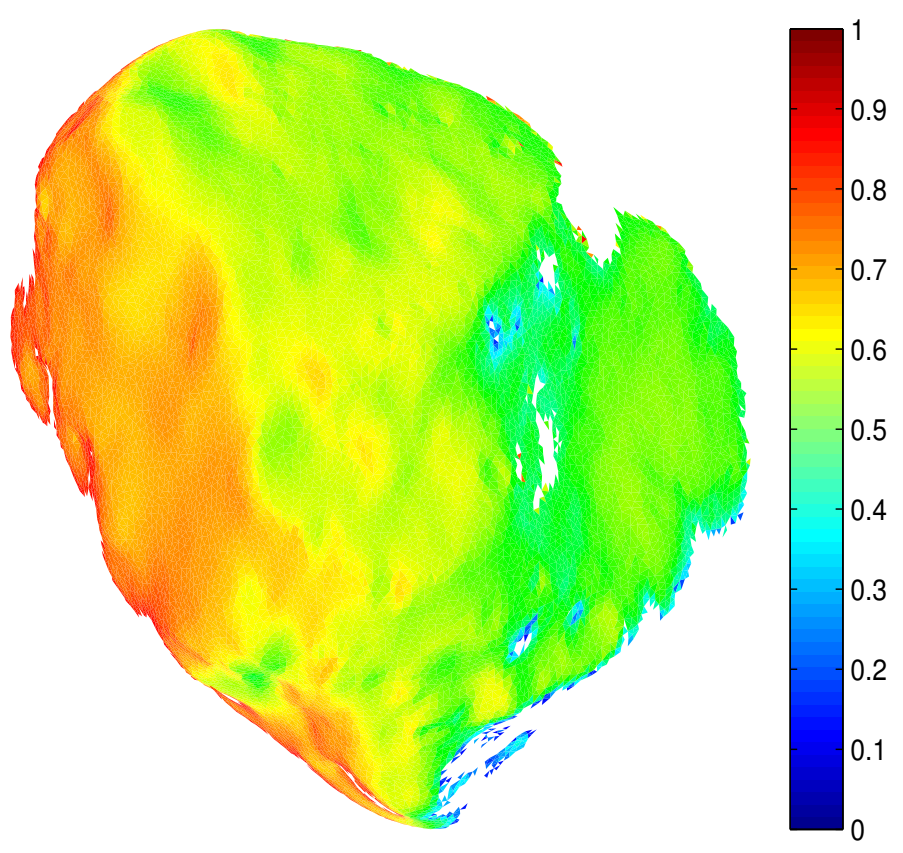

(b) $38.4^{\circ} / 26.4^{\circ}$

Figure 5.22: Simulated Phase ratio maps for Lutetia. Panel (a) for phase angles $50.4^{\circ}$ and $31.7^{\circ}$, panel (b) for $38.4^{\circ}$ and $26.4^{\circ}$ (Masoumzadeh et al., 2015) . 
of craters (NPCC) is located. The approach of the shape model alignment with images by visual inspection limits further investigation of smaller area variations. However, on a large scale we do not find any correlation between the color ratio maps and the geological terrain map of Lutetia.

The color ratio of $931.9-\mathrm{nm} / 269.3-\mathrm{nm}$ is measure of the color slope over the whole spectral interval. The symmetrical shape of histogram with the mean value of 1.14 in this color ratio (Figure 5.25) suggests uniform color variation across the surface. The FWHM of the distribution is $10 \%$ indicates the subtle color variations for Lutetia. 


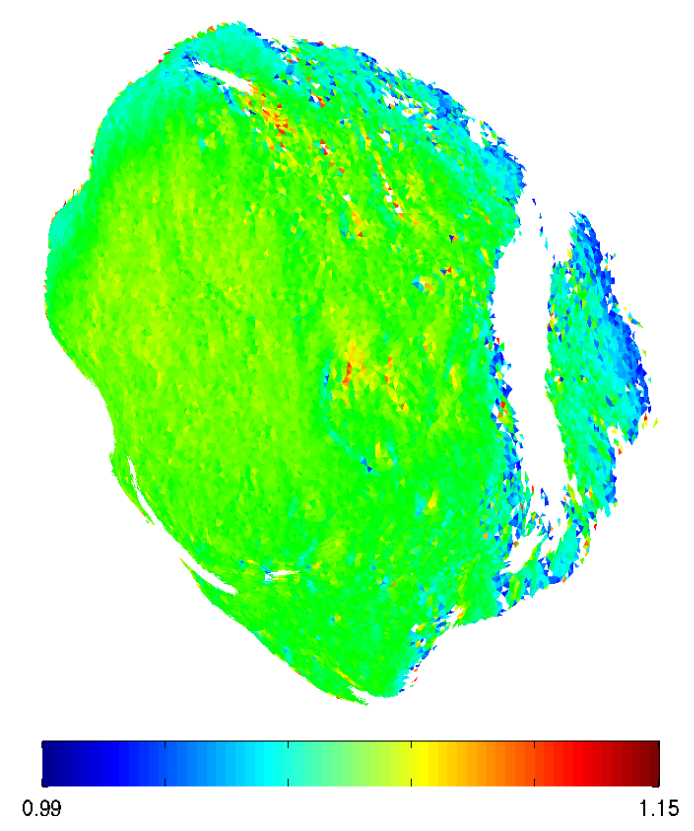

(a)

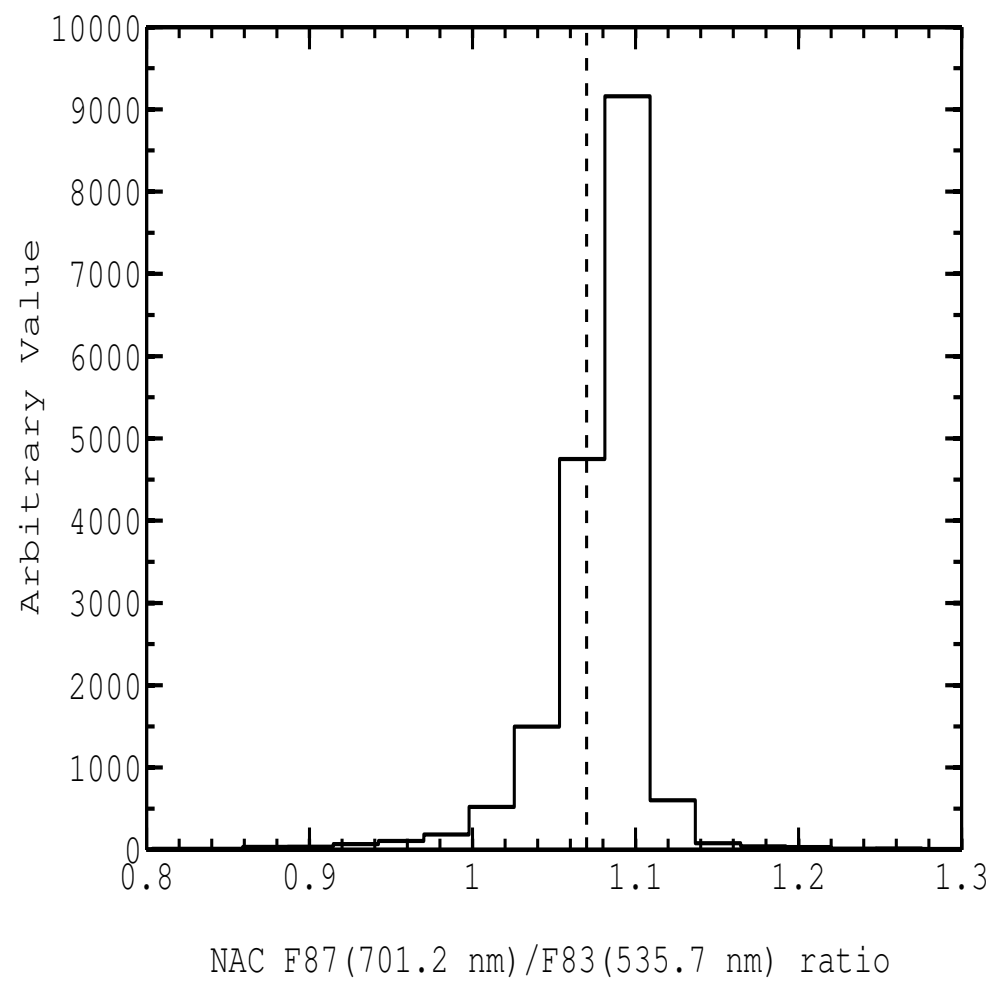

(b)

Figure 5.23: Panel(a) the color ratio histogram obtained from the NAC F87(701.2 $\mathrm{nm}) / \mathrm{F} 83(535.7 \mathrm{~nm})$ ratio. The shape model associated with NAC F87 image at phase angle of $18.5^{\circ}$ oriented at the current time of 2010-07-10T15:38:42.495 is used. Panel(b) shows the corresponding histogram of color ratios with the mean value of $1.07 \pm 0.08$. 


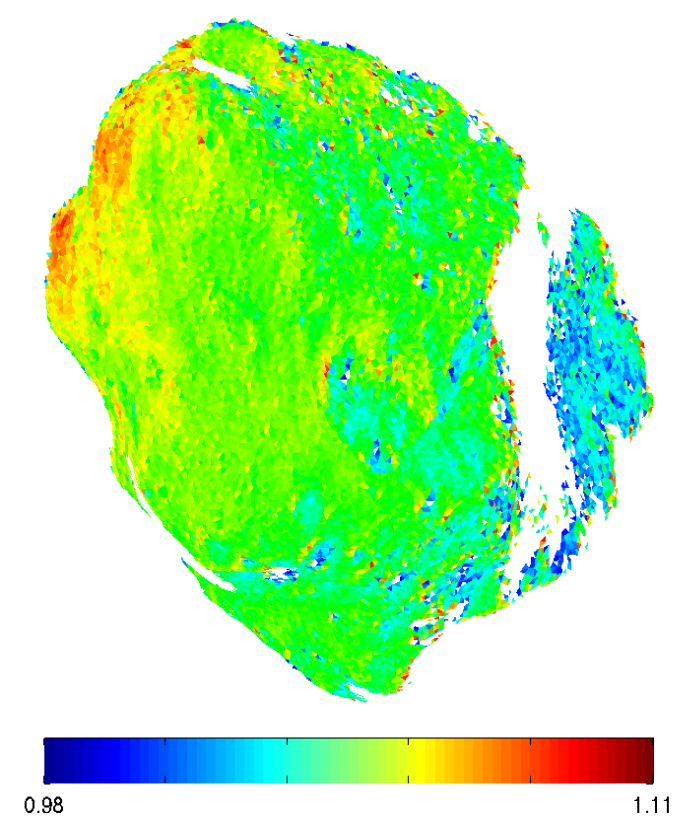

(a)

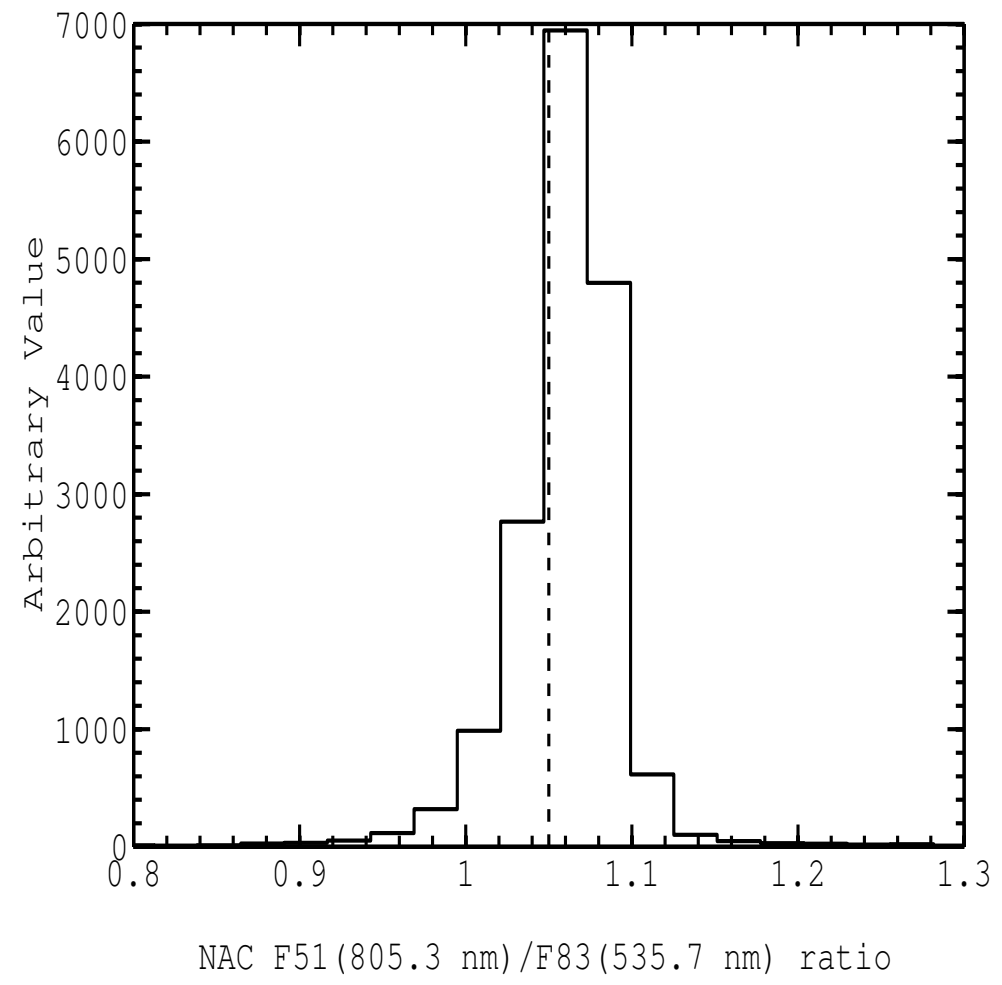

(b)

Figure 5.24: Panel(a) the color ratio map obtained from the NAC F51(805.3 $\mathrm{nm}) / \mathrm{F} 83(535.7 \mathrm{~nm})$ ratio. The shape model associated with NAC F51 image at phase angle $19.6^{\circ}$ oriented at the current time of 2010-07-10T15:38:59.537 is used. Panel(b) shows the corresponding histogram of color ratios with the mean value of $1.05 \pm 0.07$. 


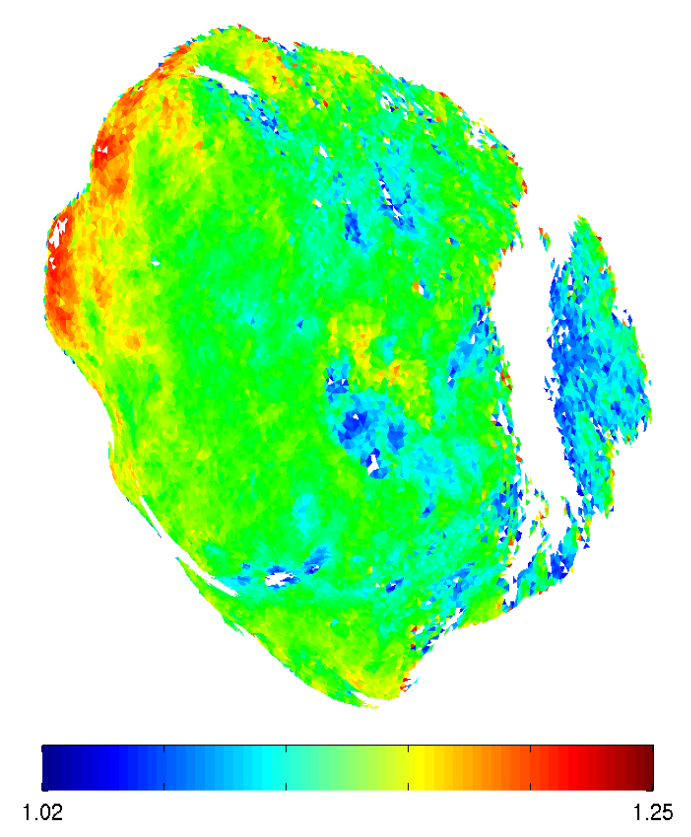

(a)

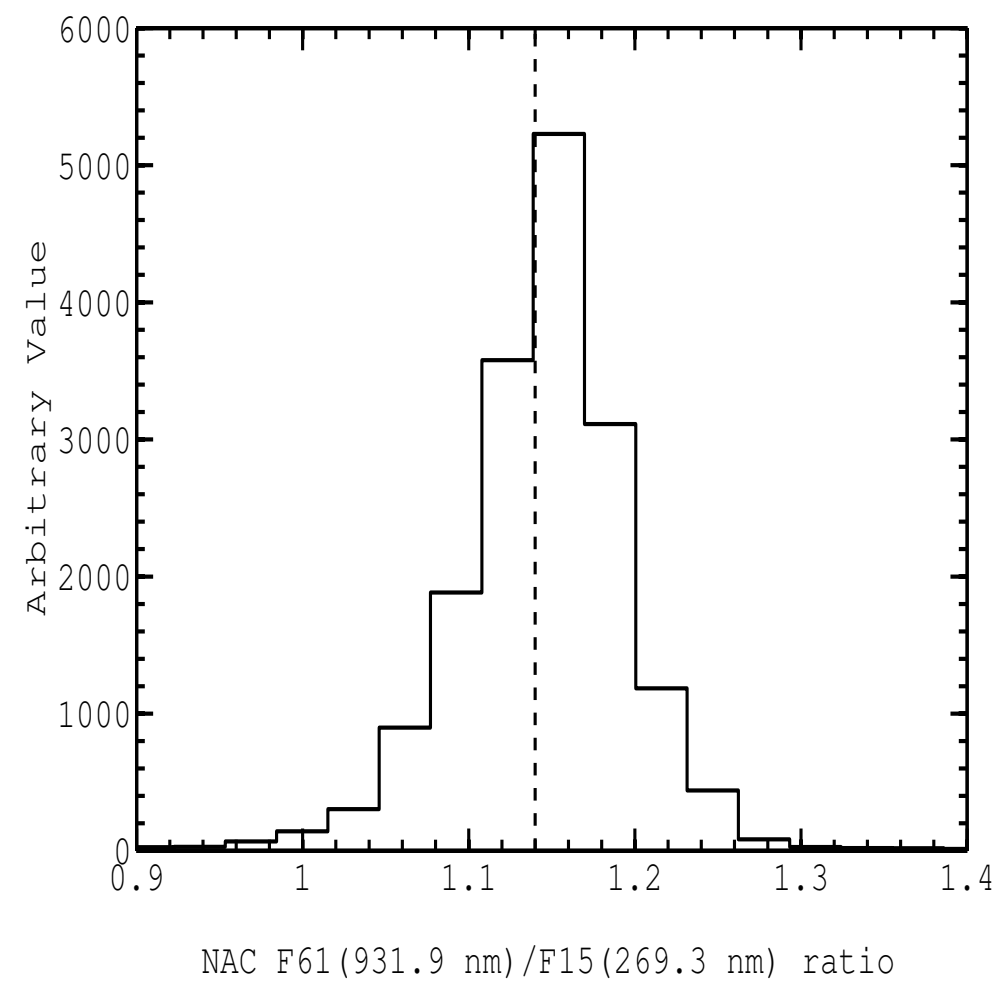

(b)

Figure 5.25: Panel (a) The illustration of color ratio map from the NAC F61(931.9 $\mathrm{nm}) / \mathrm{F} 15(269.3 \mathrm{~nm})$. The shape model associated with the NAC F61 image at phase angle of $20.8^{\circ}$ oriented at the current time of 2010-07-10T15:39:15.489 is used. Panel(b) The corresponding histogram of color ratios with the mean value of $1.14 \pm 0.12$. 



\section{Comparison of Asteroids Steins and Lutetia with other small bodies}

In this chapter, I review the photometric properties of small bodies resolved by the spacecraft images and I collate with what I found about the surface reflectance of asteroids Steins and Lutetia in chapter 4 and chapter 5 , respectively.

\subsection{Overview of small bodies' surface reflectance}

The photometric behavior of asteroids Gaspra and Ida was studied by Helfenstein and collaborators in two papers (Helfenstein et al., 1994, 1996), using Galileo images. The modeled Hapke parameters of the S-type asteroid, Gaspra, are $\omega=0.36 \pm 0.07$, $B_{0}=1.63 \pm 0.07, \mathrm{~h}=0.06 \pm 0.01, \mathrm{~g}=-0.18 \pm 0.04$, and $\bar{\theta}=29^{\circ} \pm 2^{\circ}$ which are somewhat different from the results of Hapke modeling for average S-type asteroids (Helfenstein and Veverka, 1989). However, the predicated geometric albedo is the same for the both Hapke solutions. The dissimilarity is investigated by photoclinometry technique and it is concluded that the Hapke parameters of Gaspra may represent an improvement of Hapke solutions for typical S-type asteroids. The albedo contrast of Gaspra is found to be not more than $10 \%$. The color variations over the surface of Gaspra were studied by generating color ratio maps, using images in three filters $(0.41 \mu \mathrm{m}, 0.56 \mu \mathrm{m}$ and $0.99 \mu \mathrm{m})$. A color contrast of about $5 \%$ is seen over all color filters. A distinct color heterogeneity is significant on the surface of Gaspra (Figure 6.1). Color ratio variations for $0.99 \mu \mathrm{m}$ versus $0.41 \mu \mathrm{m}$ are found to associate with local elevation, being largest with decreasing slope and smaller with increasing slope. Two different terrain units were detected that are brighter and darker in comparison with the average color variation of Gaspra. The bright materials are located along the ridges, indicating a strong 1- $\mu \mathrm{m}$ absorption, while dark materials are positioned on steep slopes with a weaker sign of the $1-\mu \mathrm{m}$ absorption.

The Hapke parameters of Ida are $\omega=0.22, \mathrm{~B}_{0}=1.53 \pm 0.10, \mathrm{~h}=0.020 \pm 0.005, \mathrm{~g}=-$ $0.33 \pm 0.01$, and $\bar{\theta}=18^{\circ} \pm 2^{\circ}$. They are more comparable with those of typical S-type asteroids than the ones of Gaspra. Unlike Gaspra, the analysis of Ida photometry indicates that the overall light scattering behavior is rather uniform across the surface and only slight variability is found in the color contrast of the Ida's surface (Veverka et al., 1996). The surface can be divided into two regions based on the 1- $\mu \mathrm{m}$ absorption (Figure 6.1). Terrain $\mathrm{A}$ is redder than average with a relatively shallower $1-\mu \mathrm{m}$ absorption, while Terrain $B$ is bluer with a deeper $1-\mu \mathrm{m}$ absorption. These terrains exhibit minor photometric differences. The photometric behavior of Terrain A is very similar to the average of Ida, while Terrain B is brighter and with higher $\omega$. 

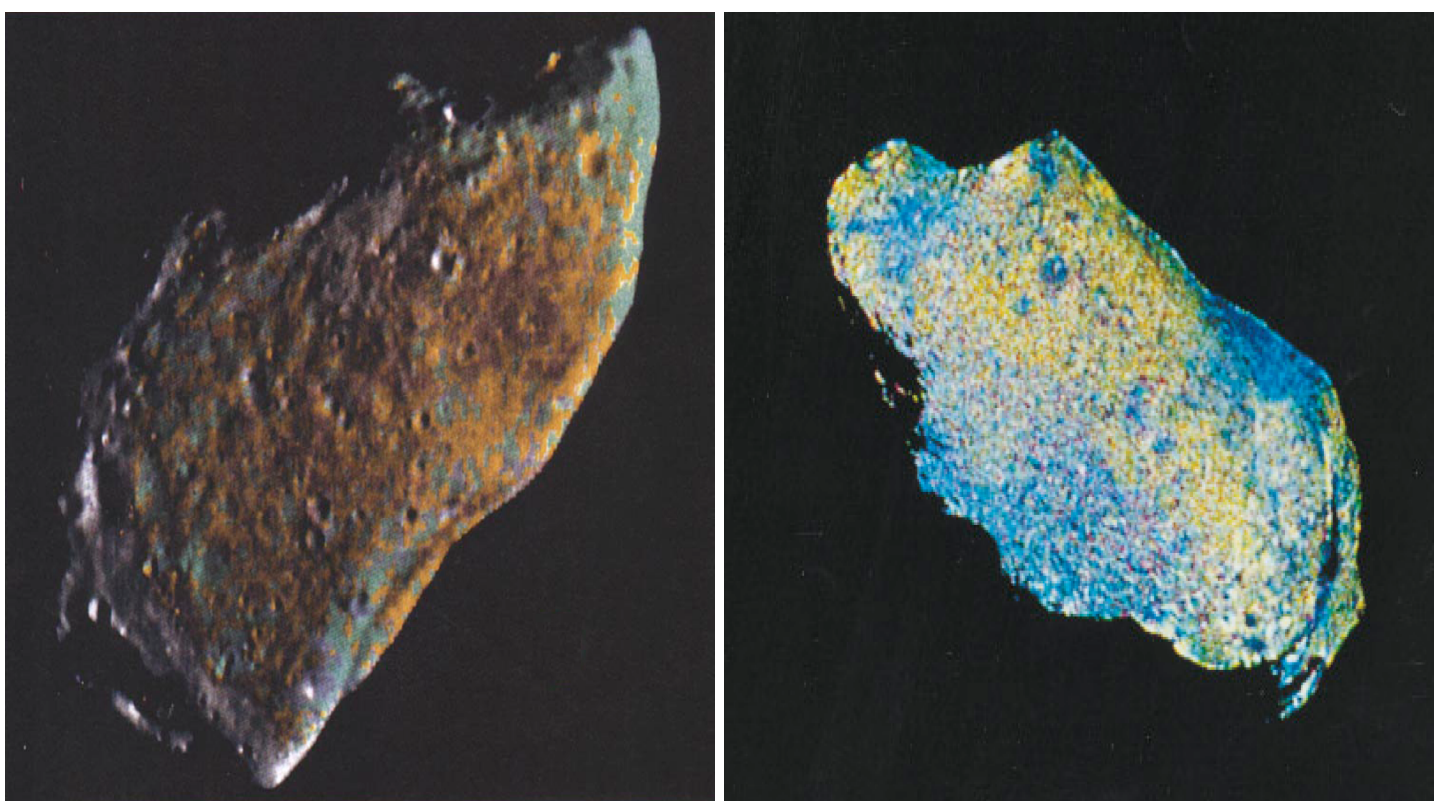

Figure 6.1: Left panel displays the color units on the surface asteroid of Gaspra based on two color ratios of $0.41-\mu \mathrm{m} / 0.56-\mu \mathrm{m}$ and $0.99 \mu \mathrm{m} / 0.56 \mu \mathrm{m}$. Following Helfenstein et al., 1994. Gaspra are formed with four classes of materials according to the level of color ratios. These four classes are shown by different colors: dark units with high ratio of $0.99-\mu \mathrm{m} / 0.56-\mu \mathrm{m}$ are reddish, intermediate units with a moderate ratio of $0.99-\mu \mathrm{m} / 0.56-$ $\mu \mathrm{m}$ are yellow, craters which are high in $0.41-\mu \mathrm{m} / 0.56-\mu \mathrm{m}$ ratio and low in $0.99-\mu \mathrm{m} / 0.56-$ $\mu \mathrm{m}$ ratio are blue and ridges are green showing small values in both the color ratios. Right panel displays a color ratio map of asteroid Ida which is constructed from three color ratios. The $0.41-\mu \mathrm{m} / 0.56-\mu \mathrm{m}$ ratio is in blue, the $0.89-\mu \mathrm{m} / 0.56-\mu \mathrm{m}$ ratio in green and the $0.99-\mu \mathrm{m} / 0.56-\mu \mathrm{m}$ ratio is red. It is evident that there are two different color units: blue regions, so called Terrain B, have a deeper 1- $\mu \mathrm{m}$ absorption and a steeper red spectral slope than the yellowish area, so called Terrain A. The figures are taken from Helfenstein et al. (1994) and Helfenstein et al. (1996).

Mathilde is the only C-type asteroid for which Hapke parameters are constrained from disk-resolved data along with disk-average data (Clark et al., 1999). The best-fit Hapke parameters of Mathilde using NEAR MultiSpectral Images (MSI) data are $\omega=0.035 \pm$ $0.006, B_{0}=3.18 \pm 1.0, \mathrm{~h}=0.074 \pm 0.003, \mathrm{~g}=-0.25 \pm 0.04, \bar{\theta}=19^{\circ} \pm 5^{\circ}$. These parameters are similar to those of average $C$-type asteroids constrained by disk-integrated phase function analysis (Helfenstein and Veverka, 1989). The surface of Mathilde is homogeneous in color and normal reflectance. No significant variation in albedo and color is seen around the 33-km crater of Mathilde. The normal reflectance variations across the surface of Mathilde are $\pm 6 \%$ from the mean.

The photometric parameters of the Near-Earth asteroids Eros and Itokawa are resembling the light scattering parameters of average S-type asteroids. The Hapke parameters of Eros are modeled in the wavelength range of 450 to $1050 \mathrm{~nm}$, using MSI images. The single scattering albedo at $550 \mathrm{~nm}$ is $0.33 \pm 0.03$. The best-fit value of the opposition surge parameters obtained from disk-integrated analysis are $B_{0}=1.4 \pm 0.1$ and $h=0.010 \pm 0.004$. The asymmetry factor and the macroscopic roughness parameters of 


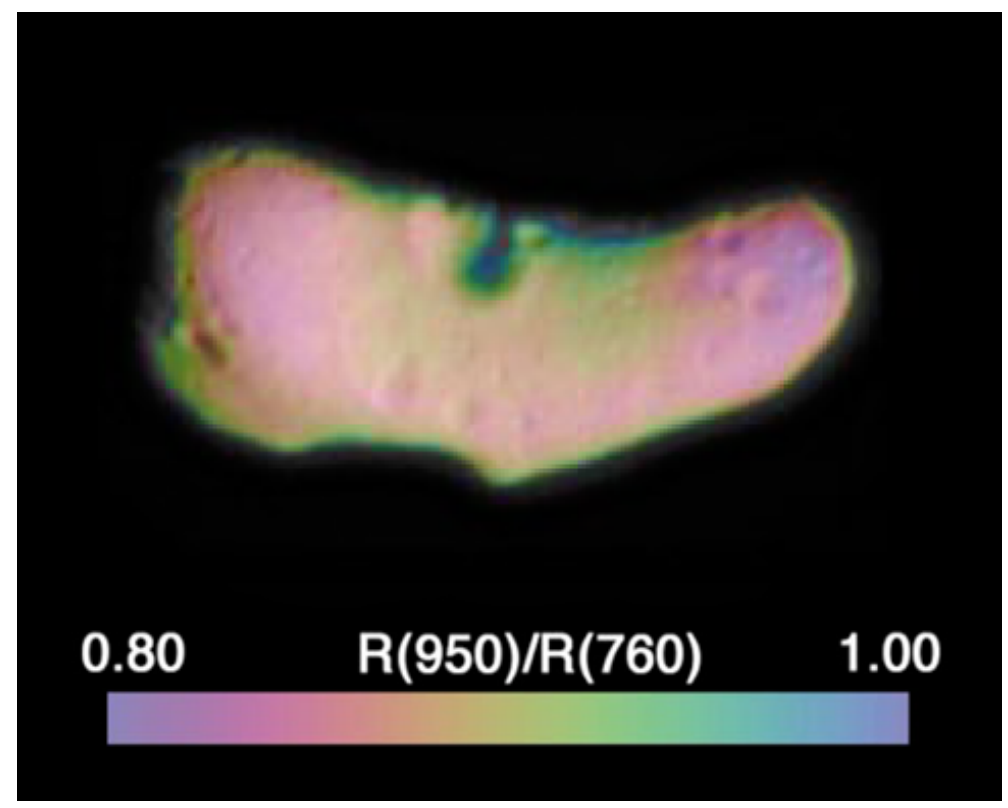

(a) $950 \mathrm{~nm} / 760 \mathrm{~nm}$ color ratio map of Eros

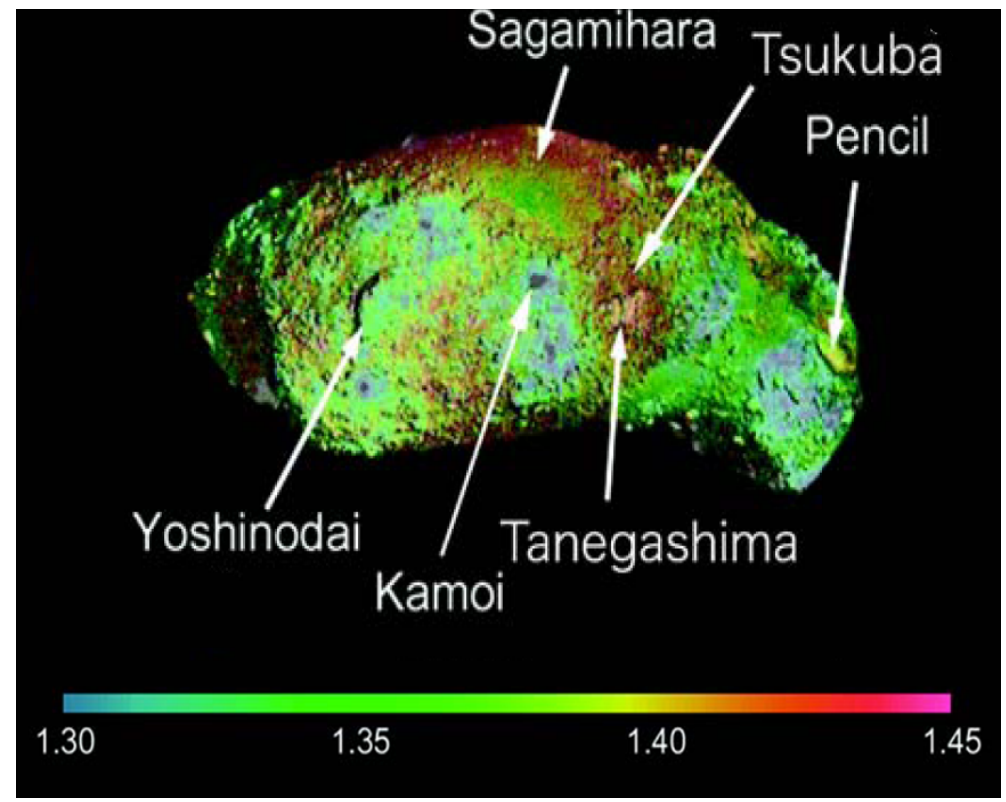

(b)

Figure 6.2: Panel (a) displays the $950 \mathrm{~nm} / 760 \mathrm{~nm}$ color ratio image of Eros which is generated by Murchie et al. (2002) to examine the 1- $\mu \mathrm{m}$ absorption depth. Significant color contrast is seen near the crater Psyche in the northern region of Eros. Panel(a) is taken from Murchie et al. (2002). Panel (b) displays the false-color ratio image of 0.70$\mu \mathrm{m} / 0.43-\mu \mathrm{m}$ for Itokawa. Two different regions of red and blue are found based on the color ratio. The text displays the names of regions on Itokawa. Panel (b) is taken from Ishiguro et al. (2007). 
Eros are independent of wavelength with the values of $-0.25 \pm 0.02$ and $28^{\circ} \pm 3^{\circ}$ respectively (Li et al. 2004). The color variation is relatively small across the surface of Eros in the visible-wavelength region; however, the color ratio map 950-nm/760-nm shows about $10 \%$ contrast (Figure 6.2). Compared to the color variation, the range of variation in reflectance is large. In comparison with other S-type asteroids such as Gaspra and Ida, it is larger by factor of two. There is a high degree of correlation between albedo and color contrast across the surface of Eros with craters, which work entirely different for Gaspra and Ida (Murchie et al., 2002).

The Hapke parameters of asteroid Itokawa are modeled by Kitazato et al. (2008) at $\lambda=1570 \mathrm{~nm}$. The macroscopic roughness is estimated to be $26^{\circ} \pm 1^{\circ}$. The other modeled Hapke parameters of Itokawa are $\omega=0.42, B_{0}=0.87, \mathrm{~h}=0.01$, and $\mathrm{g}=-0.35$. The surface of Itokowa are studied by Ishiguro et al. (2007) in the context of space weathering. The color ratio map $0.70-\mu \mathrm{m} / 0.43-\mu \mathrm{m}$ exhibits sharp color contrasts as well as two distinct areas on the surface of Itokawa a bright blue region and a dark red region (Figure 6.2). It is believed that the blue terrain contains 'fresh' material, while the red terrain is considered to consist of older material. The relationship between color and albedo variations on Itokawa is more comparable to the main belt S-type asteroids, Gaspra and Ida, to than Eros, a S-type asteroid in near-Earth orbit.

The overall light scattering behavior and albedo variation of asteroid Vesta is analyzed by Li et al. (2013c). The modeled global Hapke parameters of Vesta are: $\omega=0.050$ at 554 $\mathrm{nm}$ and 0.54 at $748 \mathrm{~nm}, g=-0.24 \pm 0.01$ and $\bar{\theta}=18^{\circ} \pm 4^{\circ}$. The values of opposition surge , $B_{0}$ and h, were fixed to 1.7 and 0.07 , respectively. The overall variation in albedo of Vesta are detected to be $17 \%$. However, the diversity of reflectance variations is evident across the surface. The largest impact crater in the southern hemisphere of Vesta, the Rheasilvia basin, is about $10 \%$ brighter than the average and in comparison with the northern hemisphere, it is $18 \%$ brighter. The darkest region on Vesta has a radiance factor of 0.08-010, while the brightest region, located on the wall of one crater, is brighter by a factor of 2 than the average.

The color variations on Vesta are unique among asteroids imaged so far by spacecraft (Reddy et al., 2012). Based on the color ratios, several types of surface colors on Vesta are detected. The color units include dark, bright, orange materials and the background called gray. The Dark terrains with low albedo show weak pyroxene absorptions, while the bright regions have deeper $0.90-\mu \mathrm{m}$ pyroxene absorption. The gray material mostly covered the surface of Vesta with a $0.75-\mu \mathrm{m}$ reflectance of $15 \%$ to $30 \%$. The materials on the surface of Vesta are distinguished by Clementine RGB (Red, Green, Blue) color ratio maps $\mathrm{R}(0.75) / \mathrm{R}(0.45), \mathrm{R}(0.75) / \mathrm{R}(0.92)$, and $\mathrm{R}(0.45) / \mathrm{R}(0.75)$, as shown in Figure 6.3 ; $(\mathrm{R}(\lambda)$ is the reflectance in a filter with the central wavelength of $\lambda(\mu \mathrm{m}))$. The moderate color ratio $\mathrm{R}(0.75) / \mathrm{R}(0.92)$ stands for gray material. The orange material is distinguished by the Clementine RGB color ratio maps $\mathrm{R}(0.75) / \mathrm{R}(0.45), \mathrm{R}(0.75) / \mathrm{R}(0.92)$, and $\mathrm{R}(0.45) / \mathrm{R}(0.75)$. The strong $\mathrm{R}(0.75) / \mathrm{R}(0.92)$ ratio (greener regions) indicates the area is in commend of materials with a strong $0.90-\mu \mathrm{m}$ pyroxene absorption band, whereas the strong $\mathrm{R}(0.75) / \mathrm{R}(0.45)$ ratio (redder regions) means steeper visible slopes in comparison with bluer regions (the higher color ratio $\mathrm{R}(0.45) / \mathrm{R}(0.75)$ ).

Results from the photometric analysis of four cometary nuclei are available, using both disk-averaged and disk-resolved reflectance data. Among them, Comet Borrelly exhibits large variations with respect to photometric parameters, particularly Hapke param- 

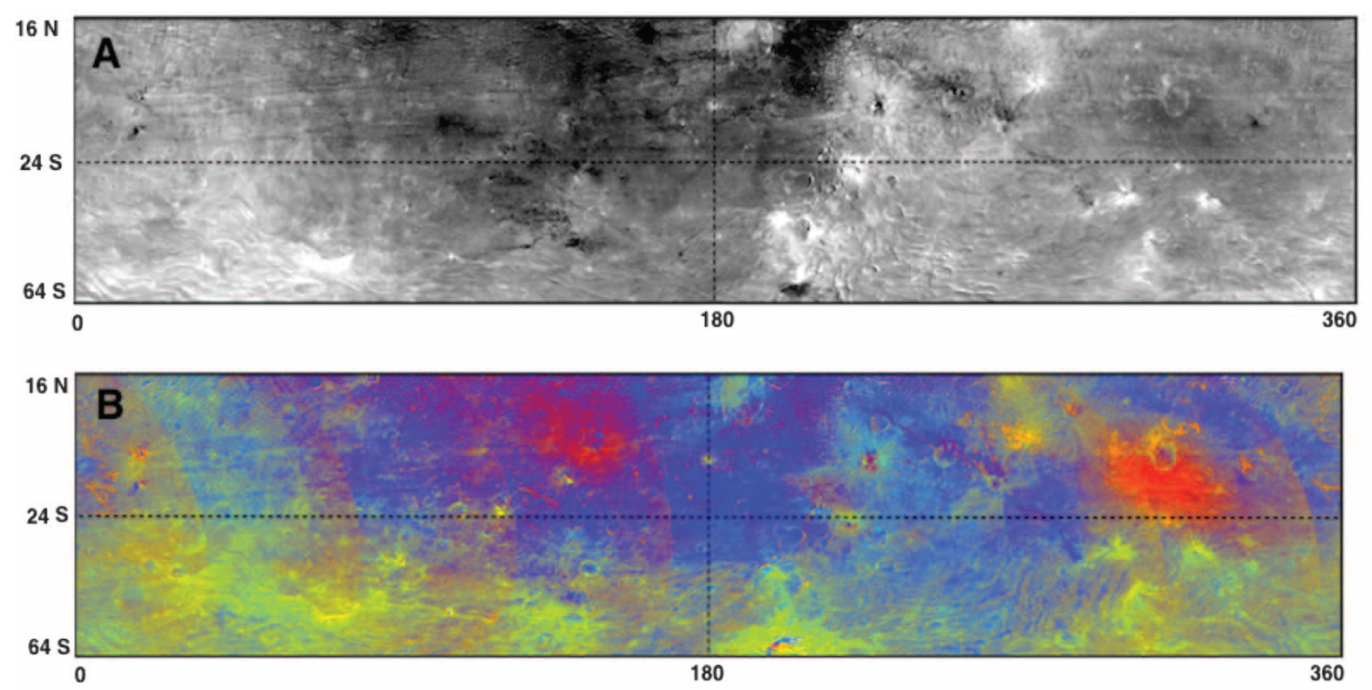

Figure 6.3: Panel (A) shows the mosaic of Vesta's surface in $0.75-\mu \mathrm{m}$ filter which is photometrically corrected and cylindrically projected. Panel (B) shows the clementine color ratio mosaic of Vesta in cylindrical projection, using three color ratios of $R(0.75) / R(0.45)$, $\mathrm{R}(0.75) / \mathrm{R}(0.92)$, and $\mathrm{R}(0.45) / \mathrm{R}(0.75)$ as a proxy for three colors of red, green and blue; respectively (taken from figure 1 in Reddy et al., 2012).

eters. The Hapke modeling performed after subdivision the terrain of Borrelly's nucleus, based on the phase ratio maps (Li et al., 2007b). The SSA $(\omega)$ is different across the surface in the range of 0.03 to 0.08 . The value of the asymmetry factor changes from weak back scattering $(-0.1)$ to strong backscattering $(-0.7)$. The roughness parameter, $\bar{\theta}$ with an average for $22^{\circ} \pm 5^{\circ}$, mostly varies below $35^{\circ}$ across the surface, except for a number of terrains at the small and big end of the nucleus. These regions are believed to be sources of jet activity seen on the surface of Borrelly because of their rather high albedo and feeble back scattering in comparison with other areas. However, for the other activity regions on Borrelly, i.e. two collimated jets, analogues light scattering properties are not found. Information as color variation of this comet is not available because all images of Borrelly are taken through one filter of $660 \mathrm{~nm}$ (Soderblom et al., 2004).

The Hapke parameters of comet 9P/Tempel 1 are modeled with $B_{0}$ and $\mathrm{h}$ are fixed to 1 and 0.01, respectively (Li et al., 2007a, 2013a). The SSA at $550 \mathrm{~nm}$ is $0.039 \pm 0.005$. The roughness parameter with the value of $16^{\circ} \pm 8^{\circ}$ does not show any wavelength dependence. The asymmetry factor of $-0.49 \pm 0.02$ is interpreted as due to a strong backscattering. The photometric variations across the nucleus of Tempel 1 are investigated by the modeled Hapke parameters at all available wavelength: the albedo varies over the surface by $20 \%$ for the FWHM of the distribution. However, some small regions are found to be indicative for water ice, being 1.8 to 2.2 times brighter than the average. The other region on Tempel 1 with slightly different photometric properties is the pitted area which shows a roughness parameter twice higher than the rest. The color variations of $3 \%$ are very small across the wavelength range covered. Some correlations between albedo variation, color variation and the geological terrains on Tempel 1 are recognized such as the morphological units and layered structures of the surface.

Disk-resolved Hapke modeling of Comet 81P/Wild 2 was also performed with fixed 
opposition effect parameters $\left(\mathrm{B}_{0}=1.0\right.$ and $\left.\mathrm{h}=0.01\right)$ due to the lack of data for small phase angles (Li et al., 2009). The best-fit value of SSA is $0.038 \pm 0.004$, the roughness parameter is equal to $27^{\circ} \pm 5^{\circ}$ and the asymmetry factor of the phase function is $-0.52 \pm 0.04$. The phase ratio maps of Wild 2 do not show any convincing variation across the surface of the nucleus. The albebo distribution shows a FWHM of 10-15\%, suggesting small variation amplitudes.

The photometric properties of the nucleus of comet 103P/Hartley 2 resulted in best-fit Hapke parameters of SSA $=0.036 \pm 0.006, \mathrm{~g}=-0.46 \pm 0.06$, and $\bar{\theta}=15^{\circ} \pm 10^{\circ}$, assuming that $\mathrm{B}_{0}$ and $\mathrm{h}$ are fixed to 1 and 0.01 , respectively (Li et al., 2013b). It was expected that a hyperactive comet like 103P/Hartley 2 exhibits large photometric variations (A'Hearn et al. 2011). However, the estimated albedo variations of $15 \%$ and color variations of $12 \%$ (ratio of 750-nm/526-nm and 750-nm/550-nm) suggest for Hartley 2 typical surface properties similar to those of Tempel 1 and Wild 2. No link is found between color ratio maps and the geological terrains on the surface of 103P. However, in inbound images a particular connection is found between the bluer bright feature near the terminator and the water ice detected by NIR spectra (Sunshine et al., 2006, Protopapa et al., 2014) in this region.

The photometric properties and variations of three cometary nuclei (Tempel 1, Wild 2, Hartley 2) are very similar with low albedo, strong back scattering and the roughness parameter in the range of $25^{\circ}-30^{\circ}$, whereas comet Borrelly exhibits large photometric variations on its surface. Therefore, the comparative evaluation of photometric variations of these four comets are not sufficient to validate the relation between light scattering parameters of the nucleus and cometary activity as found for the nucleus of Borrelly.

\subsection{Comparison between photometric properties of asteroids and cometary nuclei}

In this section, we provided a scheme to compare the modeled Minnaert parameters as well as the modeled Hapke parameters of asteroid Lutetia and Steins with those of other small bodies evaluated with these photometric models.

There are a number of small bodies modeled by the Minnaert photometric function. Since the Minnaert parameters are dependent on the phase angle $\alpha$ and wavelength $\lambda$, we compare the Minnaert parameters of small bodies estimated at zero phase angle; however, in some cases slight difference in $\lambda$ exists. According to Table 6.1, among the asteroids and cometary nuclei analyzed by the Minnaert model, all show a relatively uniform disk at zero phase angle which means without major limb-darkening, except Steins with the kvalue larger than 0.5 at opposition. In fact, the limb-darkening coefficient 0.5 represents a disk which is not limb-darkened and resembles the moon (Helfenstein and Veverka, 1987). The Minnaert albedo at opposition $\mathrm{A}_{0}$ for nearly all small bodies listed in Table 6.1 is somewhat lower than the geometric albedo, since the modeling is done excluding an opposition surge.

The comparison of Hapke parameters of Lutetia and Steins with those obtained for asteroids and cometary nuclei visited by spacecraft shows similarities and diversities among the small body population in the solar system (see Table 6.2). To make Table 6.2 easier to understand, the parameters $\mathrm{B}_{0}, \mathrm{~h}, \mathrm{~g}$, and $\bar{\theta}$ are plotted against SSA (see Figure 6.4). 
Table 6.1: Modeled Minnaert parameters at opposition for the small bodies visited by spacecraft. The geometric albedo is shown by $\mathrm{A}_{p}$.

\begin{tabular}{lccccc}
\hline Object name & $\mathrm{A}_{p}$ & ${\text { Minnaert } \mathrm{A}_{0}}$ & ${\text { Minnaert } \mathrm{k}_{0}}^{\lambda}$ & $\lambda(\mathrm{nm})$ & Source \\
\hline Asteroid Lutetia & 0.198 & $0.139 \pm 0.002$ & 0.53 & 649.2 & This work \\
Asteroid Steins & 0.41 & $0.367 \pm 0.004$ & 0.59 & 631.6 & This work \\
Asteroid Vesta & 0.42 & $0.283 \pm 0.028$ & 0.53 & 554 & Li et al. $2013 \mathrm{c}$ \\
Comet 81P/Wild 2 & 0.063 & $0.043 \pm 0.004$ & 0.54 & 647 & Li et al. 2009 \\
Comet 9P/Tempel 1 & 0.056 & 0.045 & 0.54 & 550 & Li et al. 2013a \\
\hline
\end{tabular}

All Hapke parameters are modeled at similar wavelength, except the Hapke solutions of Itokawa which is evaluated in the Near-infrared.

Table 6.2: Hapke photometric parameters of asteroids and comets. Except for S- and Ctype asteroids where the results were derived from disk-integrated reflectance analysis, all data have been obtained using disk-resolved images. Data in brackets were set fixed during the fitting.

\begin{tabular}{|c|c|c|c|c|c|c|c|c|c|}
\hline Object type & Object name & Type & SSA & $\mathrm{B}_{0}$ & $\mathrm{~h}$ & $\mathrm{~g}$ & $\bar{\theta}\left({ }^{\circ}\right)$ & $\lambda(\mathrm{nm})$ & References \\
\hline Asteroid & (21) Lutetia & - & 0.23 & 1.79 & 0.041 & -0.28 & 28 & 649.2 & This work \\
\hline Asteroid & (2867) Steins & $\mathrm{E}$ & 0.62 & 0.70 & 0.024 & -0.31 & 27 & 631.6 & This work \\
\hline Asteroid & (2867) Steins & $\mathrm{E}$ & 0.66 & 0.60 & 0.027 & -0.30 & 28 & 630 & \begin{tabular}{|l|l|} 
Spjuth et al. 2012 \\
\end{tabular} \\
\hline Asteroid & Average $\mathrm{S}$ & $\mathrm{S}$ & 0.23 & 1.32 & 0.02 & -0.35 & {$[20]$} & 540 & Helfenstein and Veverka 1989 \\
\hline Asteroid & (951) Gaspra & $\mathrm{S}$ & 0.36 & 1.63 & 0.060 & -0.18 & 29 & 560 & Helfenstein et al. 1994 \\
\hline Asteroid & (243) Ida & $\mathrm{S}$ & 0.22 & 1.53 & 0.020 & -0.33 & 18 & 560 & Helfenstein et al. 1996 \\
\hline Asteroid & (433) Eros & $\mathrm{S}$ & 0.33 & 1.4 & 0.010 & -0.25 & 28 & 550 & Li et al. 2004 \\
\hline Asteroid & (25143) Itokawa & $\mathrm{S}$ & 0.42 & 0.87 & 0.01 & -0.35 & 26 & 1570 & \begin{tabular}{|l|l} 
Kitazato et al. 2008 \\
\end{tabular} \\
\hline Asteroid & Average C & $\mathrm{C}$ & 0.037 & 1.03 & 0.025 & -0.47 & {$[20]$} & 540 & Helfenstein and Veverka 1989 \\
\hline Asteroid & (253) Mathilde & $\mathrm{C}$ & 0.035 & 3.18 & 0.074 & -0.25 & 19 & 700 & \begin{tabular}{|l|l|} 
Clark et al. & 1999 \\
\end{tabular} \\
\hline Asteroid & (4) Vesta & V & 0.51 & 1.7 & 0.07 & -0.24 & 18 & 554 & Li et al. $2013 \mathrm{c}$ \\
\hline Comet & 103P/Hartley 2 & JFC & 0.036 & {$[1.0]$} & {$[0.01]$} & -0.46 & 15 & 625 & Li et al. $2013 b$ \\
\hline Comet & 9P/Tempel 1 & JFC & 0.039 & {$[1.0]$} & {$[0.01]$} & -0.49 & 16 & 550 & Li et al. $2007 \mathrm{a}$ Li et al. $2013 \mathrm{a}$ \\
\hline Comet & 19P/Borrelly & JFC & 0.057 & {$[1.0]$} & {$[0.01]$} & -0.43 & 22 & 660 & Li et al. $2007 \mathrm{~b}$ \\
\hline Comet & 81P/Wild 2 & JFC & 0.038 & {$[1.0]$} & {$[0.01]$} & -0.52 & 27 & 647 & Li et al. 2009 \\
\hline
\end{tabular}

The two asteroids Mathilde and Steins behave as unique objects according to the scatter plots of Hapke parameters (Figure 6.4). The $\mathrm{B}_{0}$ and $\mathrm{h}$ values of Mathilde are clearly very different compared to the other small bodies, particularly the opposition surge amplitude $\mathrm{B}_{0}$ of Mathilde which is estimated to be 3.5. The g-parameter of Mathilde also shows similar value to the cometary nuclei than asteroids. For Steins, the high value of SSA and low value of $\mathrm{B}_{0}$ make it a unique body among others.

In contrast, Lutetia stands out as a typical asteroid, mainly behaving similar to S-type asteroids. However, the opposition surge width of Lutetia, $\mathrm{h}=0.041$, does not resemble any group of data points in the collection of asteroids and cometary nuclei as it is obvious in Figure 6.4 ,

The roughness versus SSA plot suggests two accumulation of data points, those are greater than $24^{\circ}$ and those with the roughness value lower than $24^{\circ}$. The roughness parameter of Lutetia $28^{\circ}$, which is in the range of $25^{\circ}-30^{\circ}$, is in common with several asteroids such as Steins, Eros, Gaspra , and one comet nucleus, Wild2.

In the asymmetry factor $g$ versus SSA plot, it is noticeable that all g-parameters of 

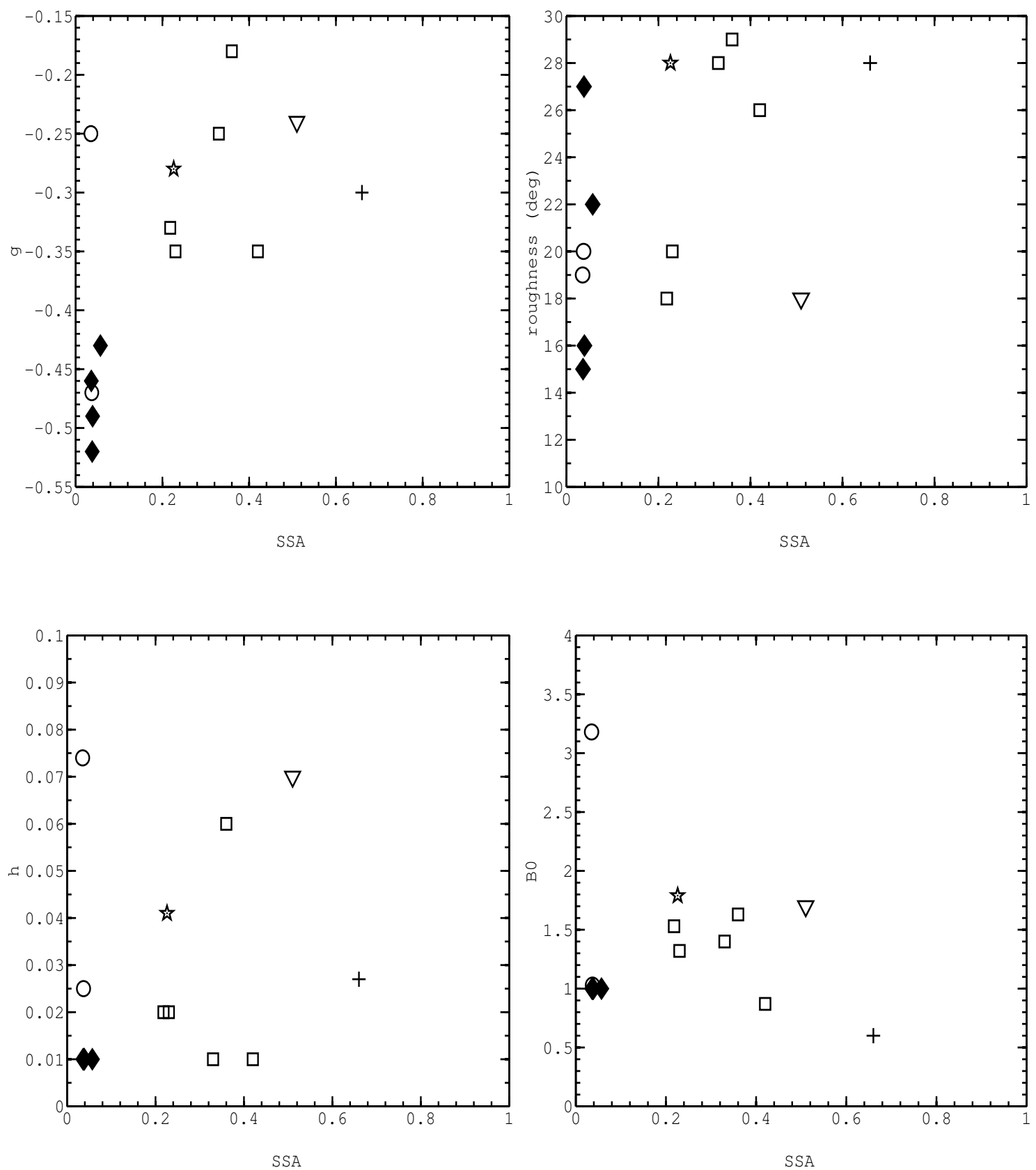

Figure 6.4: The diagrams of the four Hapke parameters $\left(\mathrm{B}_{0}, \mathrm{~h}, \mathrm{~g}\right.$, and $\left.\bar{\theta}\right)$ against the single scattering albedo (SSA) for the small bodies listed in Table 6.2. S-type asteroids are shown with open squares and C-type asteroids with circles. The corresponding points to cometary nuclei are displayed by filled diamonds. The parameters of Vesta as a member V-type asteroids are shown with triangles and for steins belongs to E-class asteroids, with crosses. The best-fit value of Hapke parameters modeled at $\lambda=649.2 \mathrm{~nm}$ for Lutetia in this thesis are depicted by star symbols. 
cometary nuclei show strong back-scattering, whereas a majority of the asteroids has a moderate value of $g$ (moderate to weak back-scattering), in the range of -0.35 to -0.15 .

Another scheme to compare Lutetia in the context of small bodies visited by spacecraft is the disk-integrated phase function. In Figure 6.5, the disk-integrated phase function of Lutetia is shown together with those of several asteroid types, based on the best-fit Hapke parameters. The overall shape of the phase function of Lutetia is closest to that of average S-type asteroids. However, the best-fit Hapke parameters of Lutetia exhibit a stronger and wider opposition effect. The phase function of average C-type asteroids is slightly steeper than typical S-type asteroids, while much deeper than the phase functions of Vesta and Steins.

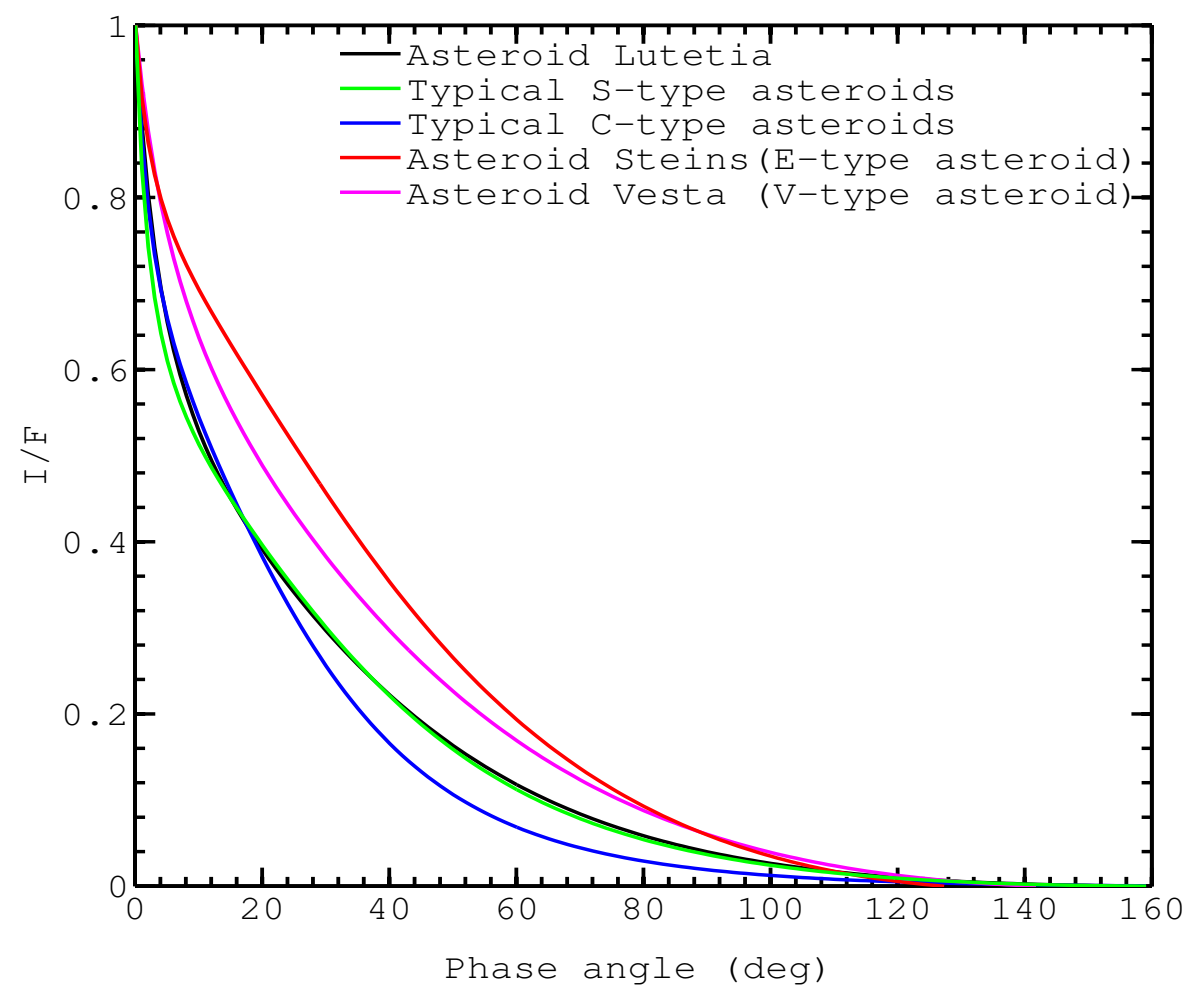

Figure 6.5: Comparison of normalized disk-integrated phase function of asteroid Lutetia with disk-integrated phase functions of asteroid Steins (Spjuth et al., 2012), asteroid Vesta (Li et al., 2013c), average S-type and C-type asteroids (Helfenstein and Veverka, 1989). The phase functions are plotted in terms of modeled Hapke parameters as tabulated in Table 6.2. and they are normalized by the value of the SSA (Masoumzadeh et al., 2015).

Considering the photometric variations across the surface of asteroids, it is worthwhile to examine the link among small bodies. The subtle albedo variation across the surface of Lutetia is similar to the reflectance variation across the surface of two different types of asteroids, Steins and Mathilde. However, no evidence of reflectance variation was found near the craters of these asteroids. The significant albedo variation is seen in the Baetica region of Lutetia at large phase angle (see chapter 5), where the North Polar Crater Cluster (NPCC) is. It is argued by Vincent et al. (2012) as an "extremely fresh" area. As it argued in chapter 5, this albedo variation is the upper limit since it appeared in the albedo ratio map at large phase angle. The same situation as the uniform albdeo variation is also 
detected for the color ratio maps of Lutetia which is very similar to the homogeneous variation in color of Steins and Mathilde.

Regarding the uniform albedo and color contrast of Steins, Mathilde, and Lutetia, the comprehensive explanations can be provided as follow; (a) the materials of interior are similar to the surface at least for the reflectance of light. Therefore, even in the location of impact craters, the excavated materials do now show different light scattering behavior compared to the surface regolith (b) the effect of space weathering is low for these bodies and the surface is not altered to cause a variation in the optical properties.

\subsection{Comparison with laboratory measurements}

In this section, we focus on the comparison of the modeled Hapke parameters of asteroids Lutetia and Steins with results from laboratory samples (terrestrial and extra terrestrial). Since the imperfection of Hapke model do not allow to interpret the physical meaning of Hapke parameters, it is self-evident that we do not expect from the photometry of meteorite to determine the meteoritic analog of asteroid. Instead we can deduce whether the light scattering behavior consistency exists between the regolith on Lutetia and the available laboratory samples. In other words, this comparison helps to constrain the albedo and the grain size of regolith covering the surface of Lutetia. The taxonomic type and mineralogical composition of Lutetia is not established yet. However, Vernazza et al. (2011) proposed enstatite chondrite meteorite for Lutetia, based on the visible, mid-infrared and near-infrared telescopic spectra. As mentioned in chapter 5, VIRITS measurements from the northern hemisphere of Lutetia also suggest two different types of meteorites, either metal-rich carbonaceous chondrite or enstatite chondrite meteorites (Coradini et al., 2011). The ground-based spectra and radar measurements also suggest a similar composition for Lutetia and the possible analogy with $\mathrm{CO} / \mathrm{CV}$ meteorites (Belskaya et al., 2010).

Among the available Hapke parameters retrieved for a number of soil and meteorite samples in the literature such as Olivine S, Olivine L, and Red Clay in Shkuratov et al. (2012), three types of basalt materials in Cord et al. (2003), 14 samples with the discrete physical properties in Shepard and Helfenstein (2007) and 8 meteorite samples being contemplated as asteroid regolith in Beck et al. (2012), we found two samples that they match with the reflectance measurement of Lutetia.

The first best-fit sample is the packed state of "Chromium Oxide" (CRp) at $\lambda=450$ $\mathrm{nm}$, examined by Shepard and Helfenstein (2007). CRp contains very fine particles $(\leq 1$ $\mu \mathrm{m}$ ) which have a tendency to form into larger unit such as aggregate of up to $1 \mathrm{~mm}$ size (Figure 6.6). The modeled Hapke parameters of CRp are: $\mathrm{SSA}=0.45, \mathrm{~B}_{0}=0.95, \mathrm{~h}=0.05$, $\mathrm{b}=0.37, \mathrm{c}=-0.58$ and $\bar{\theta}=13^{\circ}$ (Shepard and Helfenstein, 2007).

In Figure 6.7 , the disk-integrated phase function of Lutetia from WAC F17 (631.6 $\mathrm{nm})$ and NAC F22 \& F82 images $(649.2 \mathrm{~nm})$ is plotted together with the phase function of CRp sample at $\lambda=450 \mathrm{~nm}$ (CRp 450) based on its modeled Hapke parameters and in Figure 6.7b, the disk-resolved data of Lutetia in the phase angle range of $3^{\circ}$ to $63^{\circ}$ are compared with modeled I/F of CRp 450 sample. The scatter-plot in Figure 6.7p with a linear correlation coefficient of 0.93 indicate a fairly good fit, however clear systematic deviation is seen for the high $\mathrm{I} / \mathrm{F}$ values which are link to the images at $\alpha<10^{\circ}$. This 


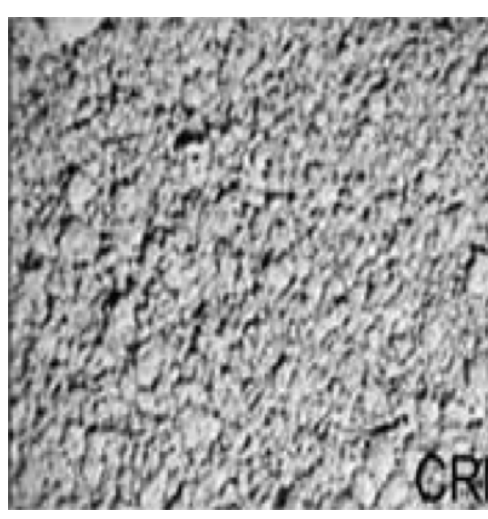

(a)

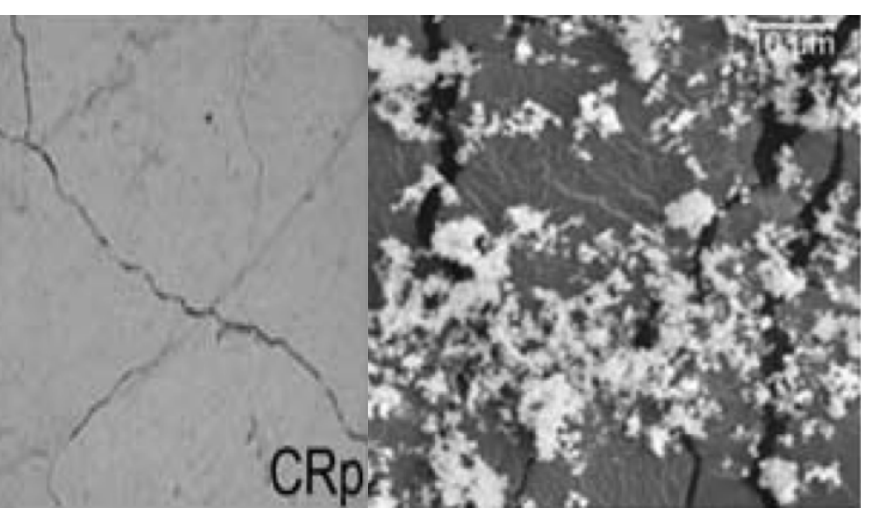

(b) (c)

Figure 6.6: Panel (a) and Panel (b) show the optical images of Chromium oxide sample (CR) in the loose (CRl) and packed (CRp) states, respectively. Panel (c) shows the scanning electron microscope image of CR. Figure is taken from Shepard and Helfenstein (2007).

similarity suggests a layer of concrete material as regolith on Lutetia.

Considering Spjuth et al. (2012)'s finding that there is a link between chromium oxide sample in loose state (CRl sample) with disk-resolved reflectance measurement of asteroid Steins. CRp and CR1 samples are similar in mean grain size but differ in bulk porosity (see Figure 6.6). The bulk porosity of CRp and CRl samples are calculated to be $71 \%$ and $84 \%$, as reported by Shepard and Helfenstein (2007). This indicates that the regolith on the surface of Lutetia is less porous than Steins. The porosity distinction also appeared in the width of opposition effect $h$ of Lutetia $(h=0.050 \pm 0.003)$ and Steins $(h=0.024 \pm 0.002)$. Considering the relation between $h$ and porosity as described in chapter 2 , the higher $h$ for Lutetia suggests lower porosity $\mathrm{p}$ on its surface in comparison to Steins.

The second one is "Allende" meteorite. Allende belongs to the CV3 carbonaceous chondrites. This sample is analyzed by Beck et al. (2012) in such a form that resemble the surface of asteroids (see Figure 6.9). The analysis reported the average grain size of roughly higher than $150 \mu \mathrm{m}$, which is distributed uniformly. The Hapke parameters of Allende sample derived from Beck et al. (2012)'s experiment are: $\mathrm{SSA}=0.357, \mathrm{~B}_{0}=1.00$, $\mathrm{h}=0.063, \mathrm{~b}=0.259, \mathrm{c}=-0.067, \bar{\theta}=3.59^{\circ}$.

The measurements are given by Beck et al. (2012) in reflectance factor (RF) unit, which differ from the radiance factor by cosine of the incidence angle $\left(\mu_{0}\right)$. Therefore, we can only compared the disk-resolved data of Lutetia with their reflectance measurement adjusting $\mu_{0}$ based on the shape model of Lutetia.

Considering the $\mu_{0}$ correction, Figure 6.10 displays the scatter plot of the modeled reflectance factor from the Hapke parameters versus the measured reflectance factor of Lutetia with a reasonably good linear correlation coefficient of 0.91 . The laboratory measurement is limited to the lowest phase angle range of $3^{\circ}$ and the amplitude of opposition effect $\mathrm{B}_{0}$ is fixed to 1 . For this reason, we do not include the measured data of Lutetia for $\alpha<3^{\circ}$, however, we still have points deviated from the linear fit in Figure 6.10 because of data from images at small phase angles (opposition surge range $0^{\circ}$ to $10^{\circ}$ ).

The similarity of reflectance factor measurements between the Allende meteorite 


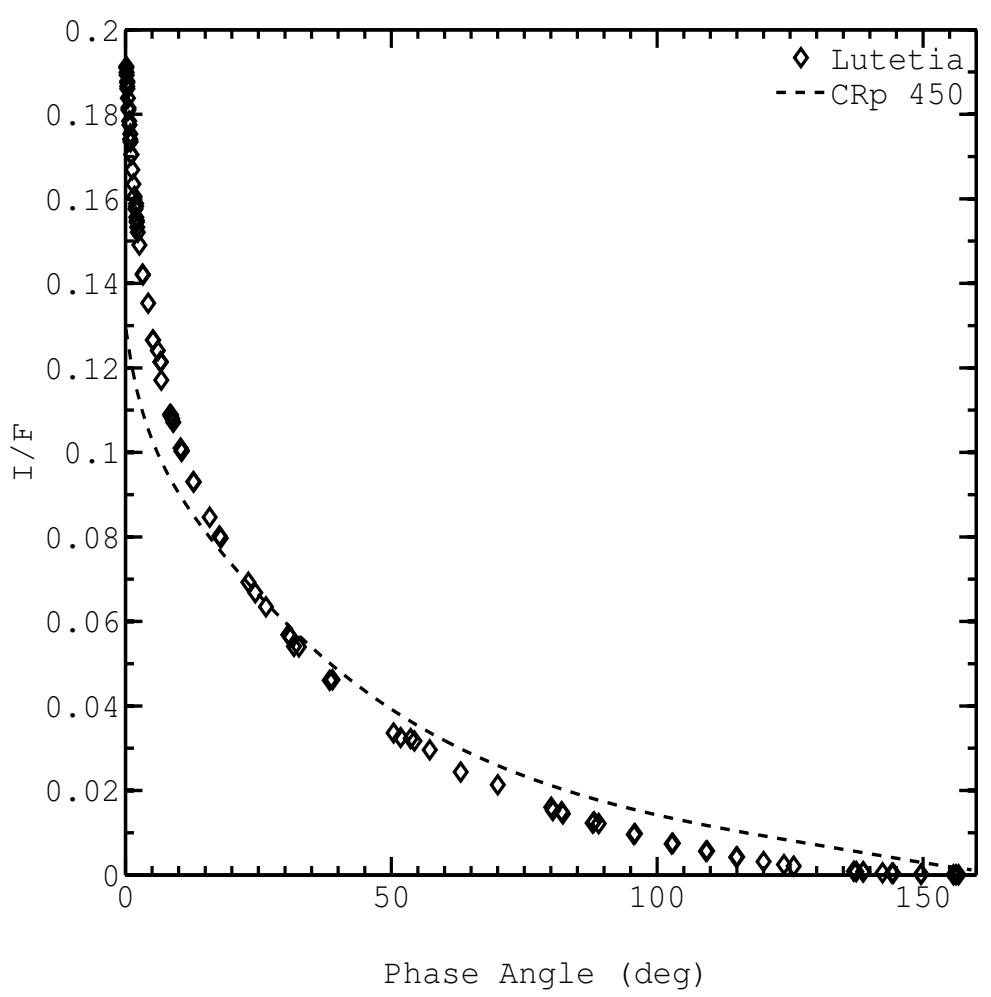

(a)

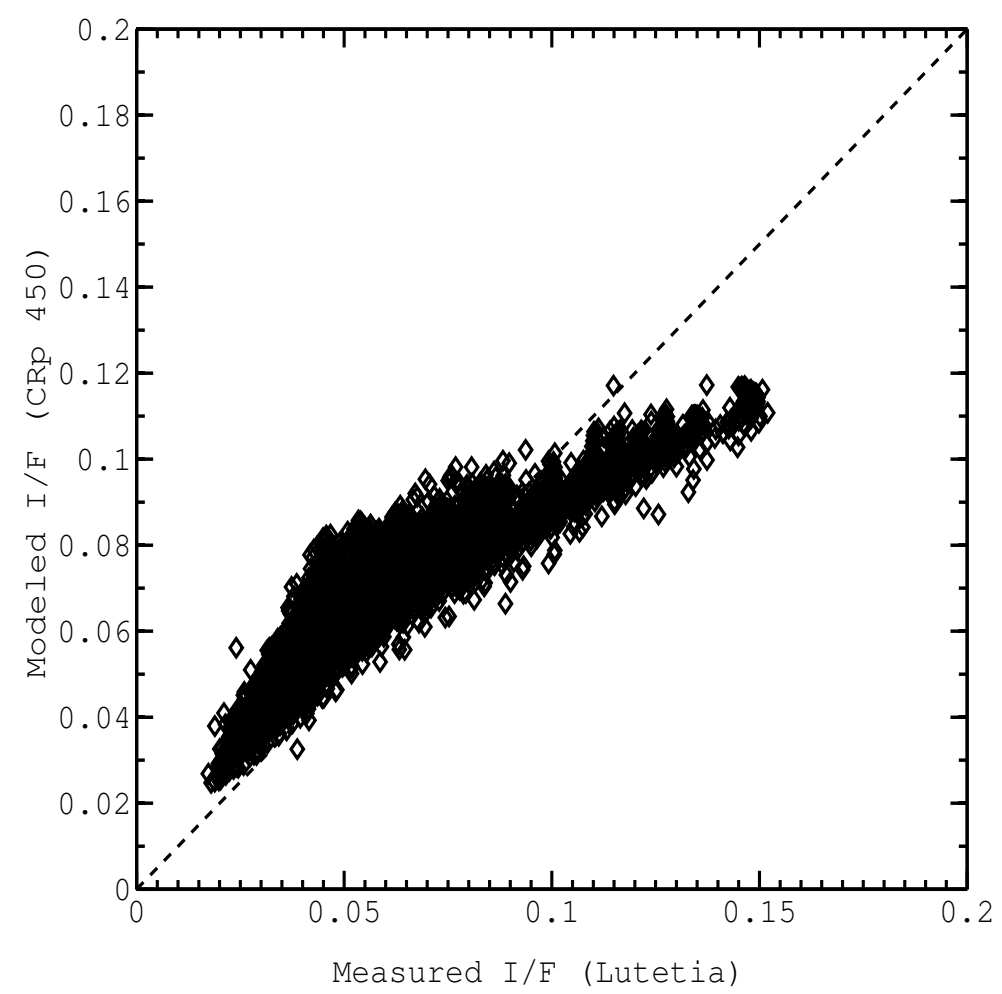

(b)

Figure 6.7: Comparison of disk-integrated and disk-resolved data of Lutetia at $\lambda=631.6$ $\mathrm{nm}$ and $649.2 \mathrm{~nm}$ with those modeled by applying Hapke parameters of the packed chromium oxide sample (CRp) in panel (a) and panel (b), respectively (Masoumzadeh et al., 2015). 
$(\lambda=650 \mathrm{~nm})$ and Lutetia at $\lambda=649.2 \mathrm{~nm}$ probably has two relevant aspects. First, the presence of carbonaceous chondrite, like Allende, is expected in the surface of Lutetia as discussed in chapter 5, especially based on the emissivity spectra of Lutetia studied by Barucci et al. (2008). The multicolor reflectance spectrum of the Allende meteorite sample obtained by Grisolle et al. (2011), displays a fairly flat reddening slope in the wavelength range of $0.4 \mu \mathrm{m}$ to $1.2 \mu \mathrm{m}$ and it shows the average reflectance is $8 \%$ brighter compared to the two other carbonaceous chondrites, Orgueil and Taglish lake used by Beck et al. (2012) in the laboratory study (Figure 6.8). Therefore, regarding the moderate albedo and the flat spectral shape of the disk-average brightness of Lutetia from OSIRIS data, we can deduce from measured reflectance that the surface of Lutetia might resemble Allende meteorite material.
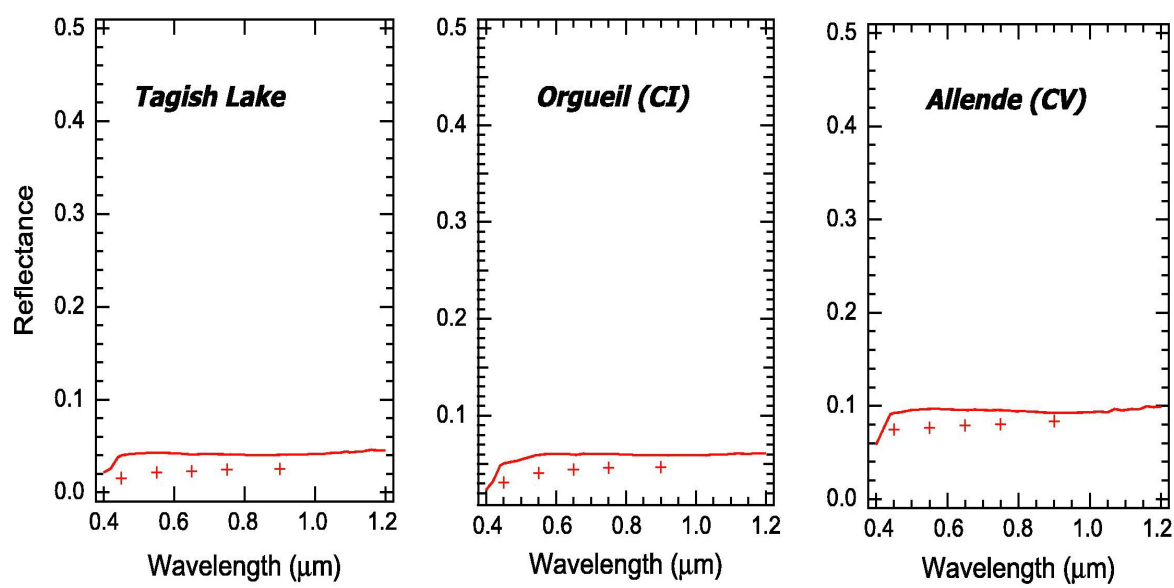

Figure 6.8: Reflectance spectra of 3 carbonaceous chondrite $(\mathrm{CC})$ meteorites powders: Tagish Lake(ungrouped CC), Orgueil(CI) and Allende(CV3) studied by Beck et al. (2012) in the spectral range of $0.4 \mu \mathrm{m}$ to $1.2 \mu \mathrm{m}$. All spectra obtained by Grisolle et al. (2011) at $\alpha=30^{\circ}$.

Secondly it is noted that the grain dimension of Allende sample $(>150 \mu \mathrm{m})$ and the grain size estimated by VIRTIS (Coradini et al., 2011) for the surface of Lutetia $\left(2.1 \pm_{1.7}^{3.4} \times\right.$ $\left.10^{2} \mu \mathrm{m}\right)$ are consistent with each other. This consistency can tell us the potential role of mechanical properties in the similarity of reflectance values.

A two-parameter $\mathrm{b}$ and $\mathrm{c}$ of the particle phase function, known as a double-term Henyey-Greenstein (2HG) phase function, described in chapter 2, can also provide general information about the properties of particles such as angular scattering, type and size of the particles. It is possible to derive the values of particle phase function parameters $b$ and $\mathrm{c}$ from the disk-integrated phase function of Lutetia since it extends to large phase angles. The $b$ and $c$ parameters represent the forward and backward lobes of scattered light from a particle. Based on the experimental study of McGuire and Hapke (1995) for three artificial types (glass, metal and polyester resin) of spherical and irregular particles with different roughness, the modeled parameters of Lutetia $b=0.33$ and $c=0.095$ are located in the middle of the $2 \mathrm{HG}$ parameters diagram (Figure 6.11). This means the regolith on Lutetia is best comparable with rough and relatively spherical resin particles. The positive value of $c$ also implies that particles on the surface of Lutetia are well back scattering with a small forward scattered lobe. In fact, the density of internal scatterers controls the 


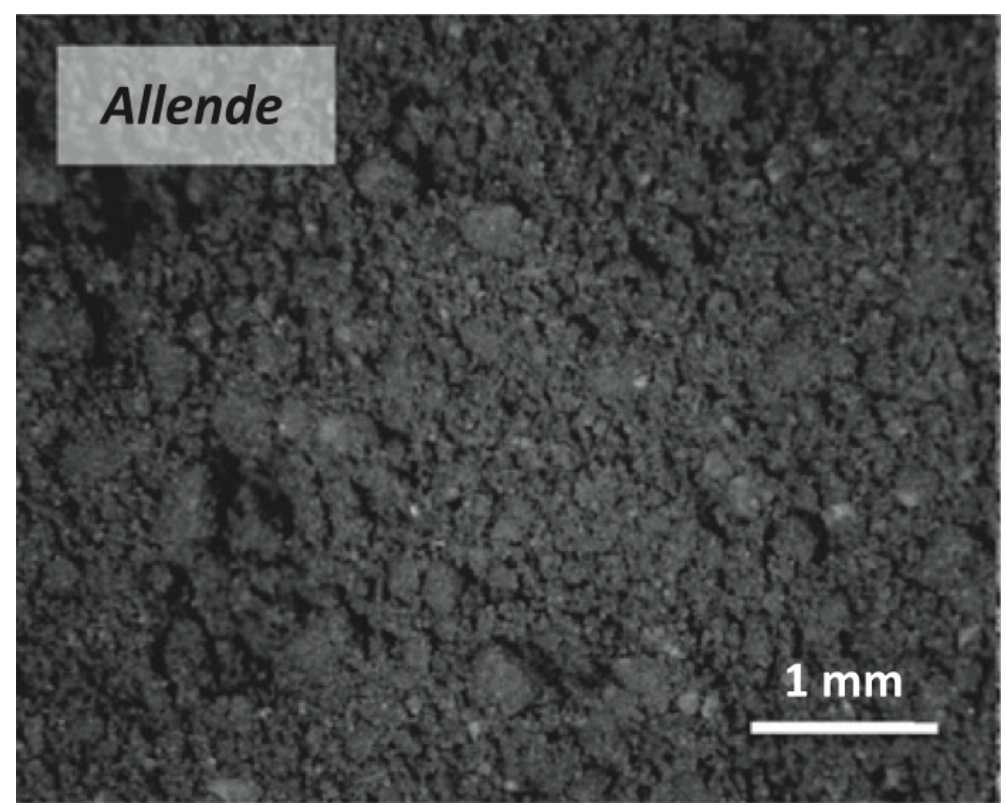

Figure 6.9: Optical microscope images of Allende powder sample from Beck et al. (2012).

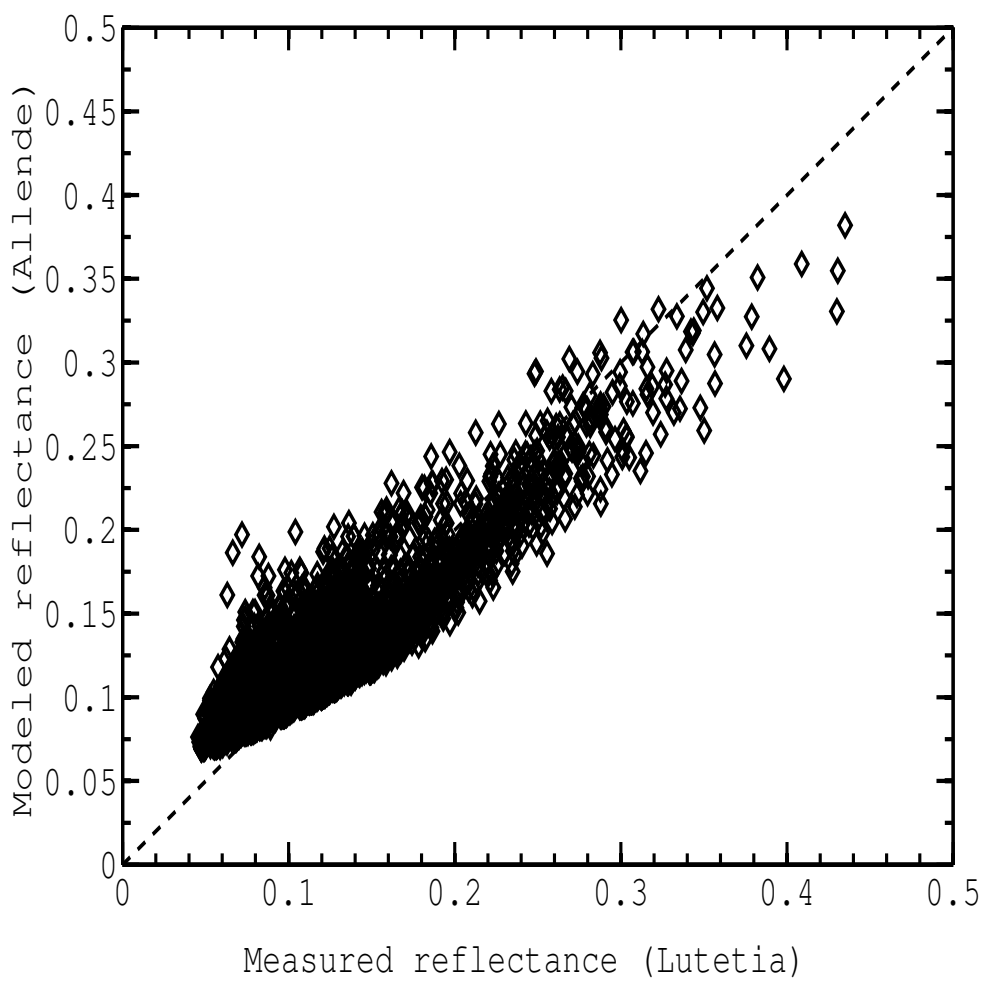

Figure 6.10: Comparison of disk-resolved data of Lutetia from NAC F82 \& F22 images at $\lambda=649.2 \mathrm{~nm}$ with those modeled by Hapke parameters of the Allende meteorite sample based on Beck et al. (2012)'s laboratory analysis through a filter with the central wavelength of $650 \mathrm{~nm}$ (Masoumzadeh et al., 2015). 
forward and backward scattering lobes. Thus, the density of internal scatterers for Lutetia is relatively moderate.

Figure 6.11 also displays the position of Steins in the 2HG parameters diagram. In comparison with Lutetia, the regolith on Steins may contain internal scatterers in large quantities (as discussed in Spjuth et al. (2012)) and it is rougher than Lutetia.

Cord et al. (2003) also conducted an experiment to constrain the two-parameter of 2HG phase function for three samples in four grain sizes (all less than $3 \mathrm{~mm}$ ) which are analogues to the planetary regolith such as fresh basalt, altered basalt and oxidized basalt materials. Based on their findings, the resulting $b$ values fall into the range of 0.4 to 0.5 and the $\mathrm{c}$ values vary between 0.13 to 0.56 for the all breeds. This suggests that the behavior of Lutetia's parameters $\mathrm{b}$ and $\mathrm{c}$ are very different from that of basaltic materials.

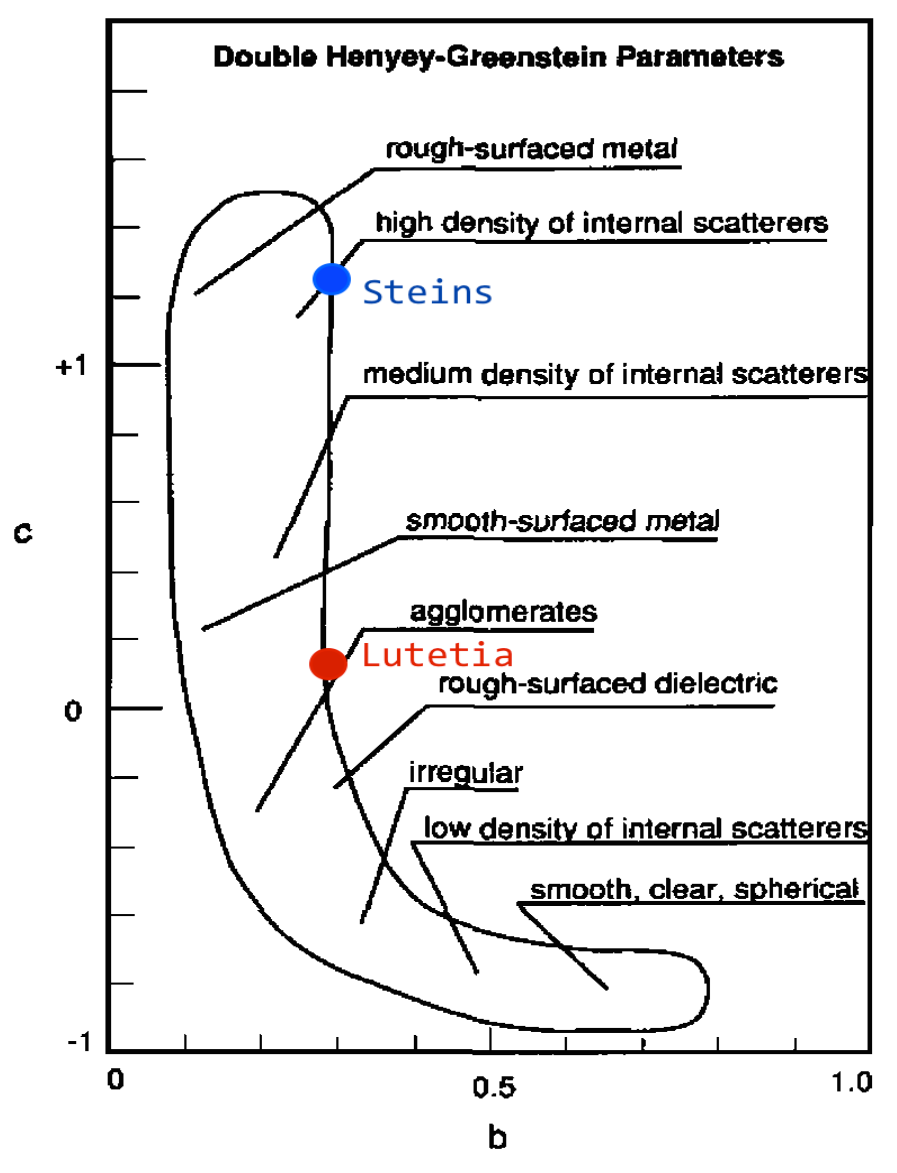

Figure 6.11: The position of asteroids Steins (blue circle) and Lutetia (red circle) in the $2 \mathrm{HG}$ parameters plot. The $2 \mathrm{HG}$ parameters diagram is taken from McGuire and Hapke (1995). 



\section{Conclusions}

Finally, in this chapter I gave a brief statement about what I have done and what I have concluded in the context of light scattering properties for two asteroids Steins and Lutetia and compared to other small bodies.

I applied two photometric functions of Minnaert and Hapke, for both asteroids Steins and Lutetia using OSIRIS measurements. I employed a direct search which is called Nelder-Mead simplex, to obtain optimal values of photometric parameters. I used the images from both OSIRIS cameras, NAC data in the wavelength range of $269.3 \mathrm{~nm}$ to $989.3 \mathrm{~nm}$ and WAC data with $\lambda$ between $295.9 \mathrm{~nm}$ to $631.6 \mathrm{~nm}$. I constructed the diskintegrated phase function of both asteroids in different filters. Those at $\lambda=631.4$ and 649.2 $\mathrm{nm}$ are the most complete ones due to the wide phase angle coverage.

I developed a tool to extract disk-resolved intensity and the corresponding geometric angles per facet from observed images, using the shape models. In order to validate my approach, I utilized the WAC images of Steins and compared my findings with the work done by Spjuth et al. (2012). The comparison indicates good agreement and it confirms the reliability of my technique.

The Minnaert modeling describes the light scattering properties of Lutetia's surface satisfactory well for phase angles $<80^{\circ}$, whereas for Steins, it represents a good fit until $\alpha=20^{\circ}$. For Lutetia, the modeled Minnaert k parameter at opposition $\left(\mathrm{k}_{0}=0.526 \pm 0.002\right)$ indicates a flat distribution in the surface brightness. No wavelength dependence is found for the $\mathrm{k}$ value of Lutetia. The $\mathrm{k}$ value of Steins, evaluated only for the phase angle dependence, is equal to $0.589 \pm 0.004$ at zero phase angle, suggesting a limb-darkened disk for the body.

I also modeled the Hapke parameters for Steins through nine filters of the WAC camera and compare my results with those of Spjuth et al. (2012). The Hapke modeling of Steins at $\lambda=631.6 \mathrm{~nm}$ yields a high single scattering albedo $(\mathrm{SSA}=0.618 \pm 0.002)$ and very low amplitude of the shadow-hiding opposition surge $\left(\mathrm{B}_{0}=0.70 \pm 0.02\right)$. The width of opposition surge is $0.024 \pm 0.002$. The asymmetry factor $g$ is estimated to be $-0.313 \pm 0.003$. The roughness parameter $\bar{\theta}$ is contained to be $27^{\circ} \pm 1^{\circ}$, which is a typical value for asteroids and agrees with the one retrieved by Spjuth et al. (2012).

Multi-wavelength values of Hapke parameters are also evaluated for Lutetia through WAC and NAC images. The SSA spectrum displays a relatively flat slope in agreement with Lutetia's spectrum as measured by VIRTIS spectrometer on-board Rosetta (Coradini et al., 2011). The best-fit value Hapke parameters of Lutetia at $\lambda=631.6 \mathrm{~nm}$ and $649.2 \mathrm{~nm}$ are as follows; The SSA is $0.226 \pm 0.002$. The opposition surge parameters $B_{0}$ is $1.79 \pm 0.08$ and $\mathrm{h}$ is $0.050 \pm 0.003$. The g-parameter of the particle phase function $\mathrm{p}(\alpha)$ is estimated to be $-0.28 \pm 0.01$. The roughness value $\bar{\theta}$ is calculated to be $28^{\circ} \pm 1^{\circ}$ in the case of NAC F82 \& F22 $(649.2 \mathrm{~nm})$ images and $24^{\circ} \pm 1^{\circ}$ for the WAC F17 $(631.6 \mathrm{~nm})$ images. The 
value of $24^{\circ}$ is consistent with the one retrieved from VIRITS data to be $23.6^{\circ}$ (Andrea Raponi, personal communication). Indeed, the spatial resolution of VIRTIS-M hyperspectral images is different from that of OSIRIS-NAC, but similar to OSIRIS-WAC, which may have resulted in different and similar roughness parameters, respectively. I noticed that in the collection of small bodies, the Hapke parameters of Lutetia are similar to those of S-type asteroids, with a rather higher opposition surge amplitude and width.

The overall light scattering variation across the surface of Lutetia is investigated by generating the albedo ratio maps, phase ratio maps and color ratio maps. The albedo variation on Lutetia is around $10 \%$ as found from albedo ratio maps of NAC F82 \& F22 $(649.2 \mathrm{~nm})$ images at small phase angles $\left(\alpha<30^{\circ}\right)$. The phase ratio maps display only small variations over the surface which may be caused by phase function or/and the photometric roughness alteration. This is confirmed by the corresponding simulated phase ratio maps. No large scale variation across the surface are found in the color ratio maps of $(805.3 \mathrm{~nm} / 535.7 \mathrm{~nm})$ and $(701.2 \mathrm{~nm} / 535.5 \mathrm{~nm})$. However, it is found that Lutetia's surface shows subtle variations in color of the order of $10 \%$ based on the color ratio map of $(931.9 \mathrm{~nm} / 269.3 \mathrm{~nm})$. It is worthy of note that the overall photometric variations of Lutetia across the surface is very similar to Mathilde (C-type asteroid) and Steins (E-type steroid).

I conduct the comparison between the Lutetia results and available laboratory reflectance measurement. Close similarity of Lutetia's light scattering behavior with reflectance measurements of two samples are found i.e. with Chromium oxide in the packed state (Shepard and Helfenstein, 2007) and with an Allende meteorite sample (Beck et al., 2012). I conclude that the similarity of photometric parameters of Lutetia with the packed chromium oxide shows that the regolith on Lutetia is dense and concrete. Lutetia's surface is also less spongy, in comparison with Steins which seems to be similar to Chromium oxide in loose state (Spjuth et al., 2012).

The consistency of Allende meteorite sample with Lutetia might indicate a similarity in the overall material constitution or at least for the size distribution to contain $150 \mu \mathrm{m}$ and larger grains. The mean grain size of Lutetia's regolith is calculated by VIRITIS measurement (Coradini et al., 2011) to be $\left(2.1 \pm_{1.7}^{3.4} \times 10^{2} \mu \mathrm{m}\right)$. Note that among available meteorite sample analyzed in the laboratory in the context of bidirectional measurement, what I found about the consistency of Allende meteorite sample (belong to subgroup CV3 of carbonaceous chondrties) and Lutetia, it does not contradict what I said about the similarity of the modeled Hapke parameters of Lutetia with average S-type asteroids because neither of them is aimed to find an analog for Lutetia. As argued in Helfenstein and Veverka (1989), the main difference between the Hapke parameters of C-type and S-type asteroids are for the SSA, not for other Hapke parameters. Therefore, since the Allende meteorite has a rather moderate albedo compared to other carbonaceous chondrtie meteorites, it resembles the Lutetia reflectance data. 


\section{Bibliography}

Accomazzo, A., Wirth, K. R., Lodiot, S., Küppers, M., Schwehm, G., 2010, The flyby of Rosetta at asteroid Steins mission and science operations, Planetary and Space Science, 58, 1058 - 1065, ISSN 0032-0633

A'Hearn, M. F., Belton, M. J. S., Delamere, W. A., Kissel, J., Klaasen, K. P., McFadden, L. A., Meech, K. J., Melosh, H. J., Schultz, P. H., Sunshine, J. M., Thomas, P. C., Veverka, J., Yeomans, D. K., Baca, M. W., Busko, I., Crockett, C. J., Collins, S. M., Desnoyer, M., Eberhardy, C. A., Ernst, C. M., Farnham, T. L., Feaga, L., Groussin, O., Hampton, D., Ipatov, S. I., Li, J.-Y., Lindler, D., Lisse, C. M., Mastrodemos, N., Owen, W. M., Richardson, J. E., Wellnitz, D. D., White, R. L., 2005, Deep Impact: Excavating comet Tempel 1, Science, 310, 258-264, http://www. sciencemag.org/content/ 310/5746/258.full.pdf

A'Hearn, M. F., Belton, M. J. S., Delamere, W. A., Feaga, L. M., Hampton, D., Kissel, J., Klaasen, K. P., McFadden, L. A., Meech, K. J., Melosh, H. J., Schultz, P. H., Sunshine, J. M., Thomas, P. C., Veverka, J., Wellnitz, D. D., Yeomans, D. K., Besse, S., Bodewits, D., Bowling, T. J., Carcich, B. T., Collins, S. M., Farnham, T. L., Groussin, O., Hermalyn, B., Kelley, M. S., Kelley, M. S., Li, J.-Y., Lindler, D. J., Lisse, C. M., McLaughlin, S. A., Merlin, F., Protopapa, S., Richardson, J. E., Williams, J. L., 2011, EPOXI at comet Hartley 2, Science, 332, 1396-1400, http: //wWw.sciencemag.org/content/332/6036/1396.full.pdf

Barucci, M., Belskaya, I., Fornasier, S., Fulchignoni, M., Clark, B., Coradini, A., Capaccioni, F., Dotto, E., Birlan, M., Leyrat, C., Sierks, H., Thomas, N., Vincent, J., 2012, Overview of Lutetia's surface composition, Planetary and Space Science, 66, 23 - 30, ISSN 0032-0633

Barucci, M. A., Fornasier, S., Dotto, E., Lamy, P. L., Jorda, L., Groussin, O., Brucato, J. R., Carvano, J., Alvarez-Candal, A., Cruikshank, D., Fulchignoni, M., 2008, Asteroids (2867) Steins and (21) Lutetia: surface composition from far infrared observations with the Spitzer space telescope, A\&A, 477, 665-670

Beck, P., Pommerol, A., Thomas, N., Schmitt, B., Moynier, F., Barrat, J.-A., 2012, Photometry of meteorites, Icarus, 218, 364 - 377, ISSN 0019-1035

Belskaya, I. N., Fornasier, S., Krugly, Y. N., Shevchenko, V. G., Gaftonyuk, N. M., Barucci, M. A., Fulchignoni, M., Gil-Hutton, R., 2010, Puzzling asteroid (21) Lutetia: our knowledge prior to the Rosetta fly-by*, A\&A, 515, A29 
Besse, S., Lamy, P., Jorda, L., Marchi, S., Barbieri, C., 2012, Identification and physical properties of craters on Asteroid (2867) Steins, Icarus, 221, 1119 - 1129, ISSN 00191035

Birlan, M., Vernazza, P., Fulchignoni, M., Barucci, M. A., Descamps, P., Binzel, R. P., Bus, S. J., 2006, Near infra-red spectroscopy of the asteroid (21) Lutetia, A\&A, 454, 677-681

Birlan, M., Nedelcu, A., Vernazza, P., Binzel, R., Carry, B., DeMeo, F., Barucci, A., Fulchignoni, M., 2010, (21) Lutetia: Groundbased near-infrared spectroscopy prior the Rosetta flyby, in AAS/Division for Planetary Sciences Meeting Abstracts \#42, vol. 42 of Bulletin of the American Astronomical Society, p. 1050

Bowell, E., Lumme, K., 1979, Colorimetry and magnitudes of asteroids, in Asteroids, (Ed.) T. Gehrels, pp. 132-169, University of Arizona Press

Brownlee, D. E., Horz, F., Newburn, R. L., Zolensky, M., Duxbury, T. C., Sandford, S., Sekanina, Z., Tsou, P., Hanner, M. S., Clark, B. C., Green, S. F., Kissel, J., 2004, Surface of young jupiter family comet $81 \mathrm{p} /$ Wild 2: View from the Stardust spacecraft, Science, 304, 1764-1769, http://www . sciencemag.org/content/304/5678/1764. full.pdf

Chandrasekhar, S., 1960, Radiative Transfer, Dover books on advanced mathematics, Dover Publications

Cheng, A. F., 2002, Near Earth Asteroid Rendezvous: Mission Summary, Asteroids III, pp. 351-366

Clark, B. E., Veverka, J., Helfenstein, P., Thomas, P., III, J. B., Harch, A., Robinson, M., Murchie, S., McFadden, L., Chapman, C., 1999, NEAR photometry of asteroid (253) Mathilde, Icarus, 140, 53 - 65, ISSN 0019-1035

Clark, B. E., Hapke, B., Pieters, C., Britt, D., 2002, Asteroid space weathering and regolith evolution, in Asteroid III, (Eds.) W. F. B. Jr., A. Cellino, P. Paolocchi, R. P. Binzel, pp. 585-599, University of Arizona Press

Coradini, A., Capaccioni, F., Drossart, P., Semery, A., Arnold, G., Schade, U., 1999, VIRTIS: The imaging spectrometer of the Rosetta mission, Advances in Space Research, 24, 1095 - 1104, ISSN 0273-1177

Coradini, A., Capaccioni, F., Erard, S., Arnold, G., De Sanctis, M. C., Filacchione, G., Tosi, F., Barucci, M. A., Capria, M. T., Ammannito, E., et al., 2011, The surface composition and temperature of asteroid (21) Lutetia as observed by Rosetta/VIRTIS, Science, $334,492-494$

Cord, A. M., Pinet, P. C., Daydou, Y., Chevrel, S. D., 2003, Planetary regolith surface analogs:: optimized determination of Hapke parameters using multi-angular spectroimaging laboratory data, Icarus, 165, 414 - 427, ISSN 0019-1035 
Forgia, F. L., Magrin, S., Bertini, I., Lazzarin, M., Pajola, M., Barbieri, C., 2012, Photometric analysis of asteroid (2867) Steins from Rosetta OSIRIS images, Memorie della Societá Astronomica Italiana, 20

Fujiwara, A., Kawaguchi, J., Yeomans, D. K., Abe, M., Mukai, T., Okada, T., Saito, J., Yano, H., Yoshikawa, M., Scheeres, D. J., Barnouin-Jha, O., Cheng, A. F., Demura, H., Gaskell, R. W., Hirata, N., Ikeda, H., Kominato, T., Miyamoto, H., Nakamura, A. M., Nakamura, R., Sasaki, S., Uesugi, K., 2006, The rubble-pile asteroid itokawa as observed by hayabusa, Science, 312, 1330-1334, http://www.sciencemag.org/ content/312/5778/1330.full.pdf

Gaffey, M. J., 2010, Space weathering and the interpretation of asteroid reflectance spectra, Icarus, 209, 564 - 574, ISSN 0019-1035

Gehrels, T., 1956, Photometric Studies of Asteroids. V. The Light-Curve and Phase Function of 20 Massalia., ApJ, 123, 331

Gradie, J., Tedesco, E., 1982, Compositional structure of the asteroid belt, Science, 216, 1405-1407, http://www. sciencemag.org/content/216/4553/1405. full.pdf

Grisolle, F., Beck, P., Quirico, E., Schmitt, B., Moynier, F., 2011, Reflectance Spectra of Carbonaceous Chondrites Under Controlled Atmosphere, Meteoritics and Planetary Science Supplement, 74, 5337

Gulkis, S., Frerking, M., Crovisier, J., Beaudin, G., Hartogh, P., Encrenaz, P., Koch, T., Kahn, C., Salinas, Y., Nowicki, R., Irigoyen, R., Janssen, M., Stek, P., Hofstadter, M., Allen, M., Backus, C., Kamp, L., Jarchow, C., Steinmetz, E., Deschamps, A., Krieg, J., Gheudin, M., Bockelee-Morvan, D., Biver, N., Encrenaz, T., Despois, D., Ip, W., Lellouch, E., Mann, I., Muhleman, D., Rauer, H., Schloerb, P., Spilker, T., 2007, MIRO: Microwave instrument for Rosetta orbiter, Space Science Reviews, 128, 561-597, ISSN 0038-6308

Gulkis, S., Keihm, S., Kamp, L., Lee, S., Hartogh, P., Crovisier, J., Lellouch, E., Encrenaz, P., Bockelee-Morvan, D., Hofstadter, M., Beaudin, G., Janssen, M., Weissman, P., von Allmen, P., Encrenaz, T., Backus, C., Ip, W.-H., Schloerb, P., Biver, N., Spilker, T., Mann, I., 2012, Continuum and spectroscopic observations of asteroid (21) Lutetia at millimeter and submillimeter wavelengths with the MIRO instrument on the Rosetta spacecraft, Planetary and Space Science, 66, 31 - 42, ISSN 0032-0633

Hampton, D., Baer, J., Huisjen, M., Varner, C., Delamere, A., Wellnitz, D., A’Hearn, M., Klaasen, K., 2005, An overview of the instrument suite for the Deep Impact mission, in Deep Impact Mission: Looking Beneath the Surface of a Cometary Nucleus, (Ed.) C. Russell, pp. 43-93, Springer Netherlands, ISBN 978-1-4020-3599-9

Hapke, B., 1966, An improved theoretical lunar photometric function, AJ, 71, 333

Hapke, B., 1981, Bidirectional reflectance spectroscopy: 1. theory, Journal of Geophysical Research: Solid Earth, 86, 3039-3054, ISSN 2156-2202 
Hapke, B., 1984, Bidirectional reflectance spectroscopy: 3. correction for macroscopic roughness, Icarus, 59, 41 - 59, ISSN 0019-1035

Hapke, B., 1986, Bidirectional reflectance spectroscopy: 4. the extinction coefficient and the opposition effect, Icarus, 67, 264 - 280, ISSN 0019-1035

Hapke, B., 1993, Theory of Reflectance and Emittance Spectroscopy, Cambridge University Press, first edn., ISBN 9781139025683

Hapke, B., 2001, Space weathering from mercury to the asteroid belt, Journal of Geophysical Research: Planets, 106, 10 039-10 073, ISSN 2156-2202

Hapke, B., 2002, Bidirectional reflectance spectroscopy: 5. the coherent backscatter opposition effect and anisotropic scattering, Icarus, 157, 523 - 534, ISSN 0019-1035

Hapke, B., 2008, Bidirectional reflectance spectroscopy: 6. effects of porosity, Icarus, 195, 918 - 926, ISSN 0019-1035

Hapke, B., Wells, E., 1981, Bidirectional reflectance spectroscopy: 2. experiments and observations, Journal of Geophysical Research: Solid Earth, 86, 3055-3060, ISSN 2156-2202

Hapke, B. W., 1963a, A theoretical photometric function for the lunar surface, Journal of Geophysical Research, 68, 4571-4586, ISSN 2156-2202

Hapke, B. W., 1963b, A theoretical photometric function for the lunar surface, Journal of Geophysical Research, 68, 4571-4586, ISSN 2156-2202

Hapke, B. W., Nelson, R. M., Smythe, W. D., 1993, The opposition effect of the Moon: The contribution of coherent backscatter, Science, 260, 509-511, http: //www.sciencemag.org/content/260/5107/509.full.pdf

Helfenstein, P., 1988, The geological interpretation of photometric surface roughness, Icarus, 73, 462 - 481, ISSN 0019-1035

Helfenstein, P., Shepard, M. K., 1999, Submillimeter-scale topography of the lunar regolith, Icarus, 141, 107 - 131, ISSN 0019-1035

Helfenstein, P., Shepard, M. K., 2011, Testing the hapke photometric model: Improved inversion and the porosity correction, Icarus, 215, 83 - 100, ISSN 0019-1035

Helfenstein, P., Veverka, J., 1987, Photometric properties of lunar terrains derived from Hapke's equation, Icarus, 72, 342 - 357, ISSN 0019-1035

Helfenstein, P., Veverka, J., 1989, Physical characterization of asteroid surfaces from photometric analysis, in Asteroids II, pp. 557-593

Helfenstein, P., Veverka, J., Thomas, P., Simonelli, D., Lee, P., Klaasen, K., Johnson, T., Breneman, H., Head, J., Murchie, S., Fanale, F., Robinson, M., Clark, B., Granahan, J., Garbeil, H., McEwen, A., Kirk, R., Davies, M., Neukum, G., Mottola, S., Wagner, R., Belton, M., Chapman, C., Pilcher, C., 1994, Galileo photometry of asteroid (951) Gaspra, Icarus, 107, 37 - 60, ISSN 0019-1035 
Helfenstein, P., Veverka, J., Thomas, P., Simonelli, D., Klaasen, K., Johnson, T., Fanale, F., Granahan, J., McEwen, A., Belton, M., Chapman, C., 1996, Galileo photometry of asteroid (243) Ida, Icarus, 120, 48 - 65, ISSN 0019-1035

Henyey, L. G., Greenstein, J. L., 1941, Diffuse radiation in the Galaxy, ApJ, 93, 70-83

Irvine, W. M., 1966, The shadowing effect in diffuse reflection, Journal of Geophysical Research, 71, 2931-2937, ISSN 2156-2202

Ishiguro, M., Hiroi, T., Tholen, D. J., Sasaki, S., Ueda, Y., Nimura, T., Abe, M., Clark, B. E., Yamamoto, A., Yoshida, F., Nakamura, R., Hirata, N., Miyamoto, H., Yokota, Y., Hashimoto, T., Kubota, T., Nakamura, A. M., Gaskell, R. W., Saito, J., 2007, Global mapping of the degree of space weathering on asteroid (25143) Itokawa by Hayabusa/AMICA observations, Meteoritics \& Planetary Science, 42, 1791-1800, ISSN 1945-5100

Jorda, L., Lamy, P., Besse, S., Capanna, C., Carry, B., Faury, G., Gaskell, R., Gesquière, G., Groussin, O., Gutiérrez, P., Kaasalainen, M., Sabolo, W., Sierks, H., Spjuth, S., OSIRIS Team, 2010, Shape and physical properties of asteroid (21) Lutetia from OSIRIS images, in AAS/Division for Planetary Sciences Meeting Abstracts \#42, vol. 42 of Bulletin of the American Astronomical Society, p. 1043

Jorda, L., Lamy, P., Gaskell, R., Kaasalainen, M., Groussin, O., Besse, S., Faury, G., 2012, Asteroid (2867) Steins: Shape, topography and global physical properties from OSIRIS observations, Icarus, 221, 1089 - 1100, ISSN 0019-1035

Keihm, S., Tosi, F., Kamp, L., Capaccioni, F., Gulkis, S., Grassi, D., Hofstadter, M., Filacchione, G., Lee, S., Giuppi, S., Janssen, M., Capria, M., 2012, Interpretation of combined infrared, submillimeter, and millimeter thermal flux data obtained during the Rosetta fly-by of asteroid (21) Lutetia, Icarus, 221, 395 - 404, ISSN 0019-1035

Keller, H., Barbieri, C., Lamy, P., Rickman, H., Rodrigo, R., Wenzel, K.-P., Sierks, H., A'Hearn, M., Angrilli, F., Angulo, M., Bailey, M., Barthol, P., Barucci, M., Bertaux, J.-L., Bianchini, G., Boit, J.-L., Brown, V., Burns, J., Büttner, I., Castro, J., Cremonese, G., Curdt, W., Deppo, V., Debei, S., Cecco, M., Dohlen, K., Fornasier, S., Fulle, M., Germerott, D., Gliem, F., Guizzo, G., Hviid, S., Ip, W.-H., Jorda, L., Koschny, D., Kramm, J., Kührt, E., Küppers, M., Lara, L., Llebaria, A., López, A., López-Jimenez, A., López-Moreno, J., Meller, R., Michalik, H., Michelena, M., Müller, R., Naletto, G., Origné, A., Parzianello, G., Pertile, M., Quintana, C., Ragazzoni, R., Ramous, P., Reiche, K.-U., Reina, M., Rodríguez, J., Rousset, G., Sabau, L., Sanz, A., Sivan, J.-P., Stöckner, K., Tabero, J., Telljohann, U., Thomas, N., Timon, V., Tomasch, G., Wittrock, T., Zaccariotto, M., 2007, OSIRIS-the scientific camera system onboard Rosetta, Space Science Reviews, 128, 433-506, ISSN 0038-6308

Keller, H. U., Schmidt, W. K. H., Wilhelm, K., Becker, C., Curdt, W., Engelhardt, W., Hartwig, H., Kramm, J. R., Meyer, H. J., Schmidt, R., Gliem, F., Krahn, E., Schmidt, H. P., Schwarz, G., Turner, J. J., Bouyries, P., Cazes, S., Angrilli, F., Bianchini, G., Fanti, G., Brunello, P., Delamere, A., Reitsema, H., Jamar, C., Cucchiaro, A., 1987, The halley multicolour camera, Journal of Physics E: Scientific Instruments, 20, 807 
Keller, H. U., Barbieri, C., Koschny, D., Lamy, P., Rickman, H., Rodrigo, R., Sierks, H., A'Hearn, M. F., Angrilli, F., Barucci, M. A., Bertaux, J.-L., Cremonese, G., Da Deppo, V., Davidsson, B., De Cecco, M., Debei, S., Fornasier, S., Fulle, M., Groussin, O., Gutierrez, P. J., Hviid, S. F., Ip, W.-H., Jorda, L., Knollenberg, J., Kramm, J. R., Kührt, E., Küppers, M., Lara, L.-M., Lazzarin, M., Moreno, J. L., Marzari, F., Michalik, H., Naletto, G., Sabau, L., Thomas, N., Wenzel, K.-P., Bertini, I., Besse, S., Ferri, F., Kaasalainen, M., Lowry, S., Marchi, S., Mottola, S., Sabolo, W., Schröder, S. E., Spjuth, S., Vernazza, P., 2010, E-type asteroid (2867) Steins as imaged by OSIRIS on board Rosetta, Science, 327, 190-193, http://www. sciencemag.org/content/ 327/5962/190.full.pdf

KenKnight, C. E., Rosenberg, D. L., Wehner, G. K., 1967, Parameters of the optical properties of the lunar surface powder in relation to solar-wind bombardment, Journal of Geophysical Research, 72, 3105-3129, ISSN 2156-2202

Kitazato, K., Clark, B. E., Abe, M., Abe, S., Takagi, Y., Hiroi, T., Barnouin-Jha, O. S., Abell, P. A., Lederer, S. M., Vilas, F., 2008, Near-infrared spectrophotometry of asteroid (25143) Itokawa from NIRS on the Hayabusa spacecraft, Icarus, 194, 137-145

Lagarias, J. C., Reeds, J. A., Wright, M. H., Wright, P. E., 1998, Convergence properties of the nelder-mead simplex method in low dimensions, SIAM Journal of Optimization, 9, 112-147

Lazzarin, M., Marchi, S., Magrin, S., Barbieri, C., 2004, Visible spectral properties of asteroid (21) Lutetia, target of Rosetta mission, A\&A, 425, L25-L28

Lazzarin, M., Marchi, S., Moroz, L. V., Magrin, S., 2009, New visible spectra and mineralogical assessment of (21) Lutetia, a target of the Rosetta mission, A\&A, 498, 307-311

Levison, H. F., Dones, L., 2007, Chapter 31 - comet populations and cometary dynamics, in Encyclopedia of the Solar System (Second Edition), (Ed.) L.-A. M. R. W. V. Johnson, pp. 575 - 588, Academic Press, San Diego, second edition edn., ISBN 978$0-12-088589-3$

Leyrat, C., Fornasier, S., Barucci, A., Magrin, S., Lazzarin, M., Fulchignoni, M., Jorda, L., Belskaya, I., Marchi, S., Barbieri, C., Keller, U., Sierks, H., Hviid, S., 2010, Search for Steins' surface inhomogeneities from OSIRIS Rosetta images, Planetary and Space Science, 58, 1097 - 1106, ISSN 0032-0633

Li, J., A'Hearn, M. F., McFadden, L. A., 2004, Photometric analysis of eros from near data, Icarus, 172, 415 - 431, ISSN 0019-1035

Li, J.-Y., A’Hearn, M. F., Belton, M. J., Crockett, C. J., Farnham, T. L., Lisse, C. M., McFadden, L. A., Meech, K. J., Sunshine, J. M., Thomas, P. C., Veverka, J., 2007a, Deep Impact photometry of comet 9p/Tempel 1, Icarus, 191, 161 - 175, ISSN 00191035

Li, J.-Y., A'Hearn, M. F., McFadden, L. A., Belton, M. J., 2007b, Photometric analysis and disk-resolved thermal modeling of comet 19p/Borrelly from Deep Space 1 data, Icarus, 188, 195 - 211, ISSN 0019-1035 
Li, J.-Y., A'Hearn, M. F., Farnham, T. L., McFadden, L. A., 2009, Photometric analysis of the nucleus of comet 81p/Wild 2 from Stardust images, Icarus, 204, 209 - 226, ISSN 0019-1035

Li, J.-Y., A’Hearn, M. F., Belton, M. J., Farnham, T. L., Klaasen, K. P., Sunshine, J. M., Thomas, P. C., Veverka, J., 2013a, Photometry of the nucleus of comet 9p/Tempel 1 from Stardust-NExT flyby and the implications, Icarus, 222, 467 - 476, ISSN 00191035

Li, J.-Y., Besse, S., A’Hearn, M. F., Belton, M. J., Bodewits, D., Farnham, T. L., Klaasen, K. P., Lisse, C. M., Meech, K. J., Sunshine, J. M., Thomas, P. C., 2013b, Photometric properties of the nucleus of comet 103p/Hartley 2, Icarus, 222, $559-570$, ISSN 00191035

Li, J.-Y., Corre, L. L., Schröder, S. E., Reddy, V., Denevi, B. W., Buratti, B. J., Mottola, S., Hoffmann, M., Gutierrez-Marques, P., Nathues, A., Russell, C. T., Raymond, C. A., 2013c, Global photometric properties of asteroid (4) Vesta observed with Dawn framing camera, Icarus, 226, 1252 - 1274, ISSN 0019-1035

Lumme, K., Bowell, E., 1981, Radiative transfer in the surfaces of atmosphereless bodies. I - Theory. II - Interpretation of phase curves, AJ, 86, 1694-1721

Magrin, S., Forgia, F. L., Pajola, M., Lazzarin, M., Massironi, M., Ferri, F., Deppo, V. D., Barbieri, C., Sierks, H., 2012, (21) Lutetia spectrophotometry from Rosetta-OSIRIS images and comparison to ground-based observations, Planetary and Space Science, 66, 43 - 53, ISSN 0032-0633

Marchi, S., Barbieri, C., Küppers, M., Marzari, F., Davidsson, B., Keller, H., Besse, S., Lamy, P., Mottola, S., Massironi, M., Cremonese, G., 2010, The cratering history of asteroid (2867) Steins, Planetary and Space Science, 58, 1116 - 1123, ISSN 0032-0633

Marchi, S., Massironi, M., Vincent, J.-B., Morbidelli, A., Mottola, S., Marzari, F., Küppers, M., Besse, S., Thomas, N., Barbieri, C., Naletto, G., Sierks, H., 2012, The cratering history of asteroid (21) Lutetia, Planetary and Space Science, 66, 87 - 95, ISSN 0032-0633

Masoumzadeh, N., Boehnhardt, H., Li, J.-Y., Vincent, J.-B., 2015, Photometric analysis of asteroid (21) lutetia from rosetta-osiris images, Icarus, 257, 239 - 250, ISSN 00191035

Massironi, M., Marchi, S., Pajola, M., Snodgrass, C., Thomas, N., Tubiana, C., Vincent, J. B., Cremonese, G., Deppo, V. D., Ferri, F., Magrin, S., Sierks, H., Barbieri, C., Lamy, P., Rickman, H., Rodrigo, R., Koschny, D., 2012, Geological map and stratigraphy of asteroid (21) Lutetia, Planetary and Space Science, 66, 125 - 136, ISSN 0032-0633

McEwen, A. S., 1991, Photometric functions for photoclinometry and other applications, Icarus, 92, 298 - 311, ISSN 0019-1035

McGuire, A. F., Hapke, B. W., 1995, An experimental study of light scattering by large, irregular particles, Icarus, 113, 134 - 155, ISSN 0019-1035 
Minnaert, M., 1941, The reciprocity principle in lunar photometry, apj, 93, 403-410

Müller, G., 1893, Helligkeitsbestimmungen der grossen Planeten u. einiger Asteroiden, Publicationen des astrophysikalischen Observatoriums zu Potsdam, Engelmann

Murchie, S., Robinson, M., Clark, B., Li, H., Thomas, P., Joseph, J., Bussey, B., Domingue, D., Veverka, J., Izenberg, N., Chapman, C., 2002, Variations on Eros from NEAR multispectral imaging, Icarus, 155, 145 - 168, ISSN 0019-1035

Newburn, R. L., Bhaskaran, S., Duxbury, T. C., Fraschetti, G., Radey, T., Schwochert, M., 2003, Stardust Imaging Camera, Journal of Geophysical Research (Planets), 108, 8116

Pater, I. D., Lissauer, J. J., 2001, Planetary Sciences, Cambridge University Press, first edn.

Perna, D., Dotto, E., Lazzarin, M., Magrin, S., Fulchignoni, M., Barucci, M. A., Fornasier, S., Marchi, S., Barbieri, C., 2010, Inhomogeneities on the surface of (21) Lutetia, the asteroid target of the Rosetta mission*, A\&A, 513, L4

Petit, J.-M., Chambers, J., Franklin, F., Nagasawa, M., 2002, Primordial excitation and depletion of the main belt, in Asteroid III, (Eds.) W. F. B. Jr., A. Cellino, P. Paolocchi, R. P. Binzel, pp. 711-723, University of Arizona Press

Pinty, B., Verstraete, M. M., Dickinson, R. E., 1989, A physical model for predicting bidirectional reflectances over bare soil, Remote Sensing of Environment, 27, 273 288, ISSN 0034-4257

Protopapa, S., Sunshine, J. M., Feaga, L. M., Kelley, M. S., A’Hearn, M. F., Farnham, T. L., Groussin, O., Besse, S., Merlin, F., Li, J.-Y., 2014, Water ice and dust in the innermost coma of comet 103P/Hartley 2, Icarus, 238, 191 - 204, ISSN 0019-1035

Reddy, V., Nathues, A., Le Corre, L., Sierks, H., Li, J.-Y., Gaskell, R., McCoy, T., Beck, A. W., Schröder, S. E., Pieters, C. M., Becker, K. J., Buratti, B. J., Denevi, B., Blewett, D. T., Christensen, U., Gaffey, M. J., Gutierrez-Marques, P., Hicks, M., Keller, H. U., Maue, T., Mottola, S., McFadden, L. A., McSween, H. Y., Mittlefehldt, D., O'Brien, D. P., Raymond, C., Russell, C., 2012, Color and albedo heterogeneity of Vesta from dawn, Science, 336, 700-704, http: //www. sciencemag.org/content/ 336/6082/700.full.pdf

Rivkin, A., Howell, E., Lebofsky, L., Clark, B., Britt, D., 2000, The nature of M-class asteroids from $3 \mu \mathrm{m}$ observations, Icarus, 145, 351 - 368, ISSN 0019-1035

Rivkin, A. S., Clark, B. E., Ockert-Bell, M., Volquardsen, E., Howell, E. S., Bus, S. J.,

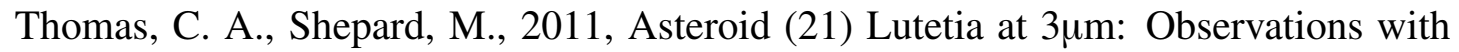
IRTF speX, Icarus, 216, 62 - 68, ISSN 0019-1035

Russell, C. T., Raymond, C. A., Coradini, A., McSween, H. Y., Zuber, M. T., Nathues, A., De Sanctis, M. C., Jaumann, R., Konopliv, A. S., Preusker, F., Asmar, S. W., Park, R. S., Gaskell, R., Keller, H. U., Mottola, S., Roatsch, T., Scully, J. E. C., Smith, 
D. E., Tricarico, P., Toplis, M. J., Christensen, U. R., Feldman, W. C., Lawrence, D. J., McCoy, T. J., Prettyman, T. H., Reedy, R. C., Sykes, M. E., Titus, T. N., 2012, Dawn at vesta: Testing the protoplanetary paradigm, Science, 336, 684-686, http://www. sciencemag.org/content/336/6082/684.full.pdf

Schröder, S., Keller, H., Gutierrez, P., Hviid, S., Kramm, R., Sabolo, W., Sierks, H., 2010, Evidence for surface variegation in Rosetta OSIRIS images of asteroid (2867)Steins, Planetary and Space Science, 58, 1107 - 1115, ISSN 0032-0633

Schulz, R., 2010, The Rosetta mission and its fly-by at asteroid (2867) Steins, Planetary and Space Science, 58, 1057 -, ISSN 0032-0633

Schulz, R., Alexander, C., Boehnhardt, H., Glassmeier, K., 2009, ESA's Mission to the Origin of the Solar System, Springer

Schulz, R., Sierks, H., Küppers, M., Accomazzo, A., 2012, Rosetta fly-by at asteroid (21) Lutetia: An overview, Planetary and Space Science, 66, 2 - 8, ISSN 0032-0633

Shepard, M. K., Campbell, B. A., 1998, Shadows on a planetary surface and implications for photometric roughness, Icarus, 134, 279 - 291, ISSN 0019-1035

Shepard, M. K., Helfenstein, P., 2007, A test of the Hapke photometric model, Journal of Geophysical Research: Planets, 112, ISSN 2156-2202

Shkuratov, Y., Kaydash, V., Korokhin, V., Velikodsky, Y., Opanasenko, N., Videen, G., 2011, Optical measurements of the moon as a tool to study its surface, Planetary and Space Science, 59, 1326 - 1371, ISSN 0032-0633

Shkuratov, Y., Kaydash, V., Korokhin, V., Velikodsky, Y., Petrov, D., Zubko, E., Stankevich, D., Videen, G., 2012, A critical assessment of the Hapke photometric model, Journal of Quantitative Spectroscopy and Radiative Transfer, 113, 2431 - 2456, ISSN 0022-4073

Sierks, H., Lamy, P., Barbieri, C., Koschny, D., Rickman, H., Rodrigo, R., A’Hearn, M. F., Angrilli, F., Barucci, M. A., Bertaux, J.-L., Bertini, I., Besse, S., Carry, B., Cremonese, G., Da Deppo, V., Davidsson, B., Debei, S., De Cecco, M., De Leon, J., Ferri, F., Fornasier, S., Fulle, M., Hviid, S. F., Gaskell, R. W., Groussin, O., Gutierrez, P., Ip, W., Jorda, L., Kaasalainen, M., Keller, H. U., Knollenberg, J., Kramm, R., Kührt, E., Küppers, M., Lara, L., Lazzarin, M., Leyrat, C., Moreno, J. J. L., Magrin, S., Marchi, S., Marzari, F., Massironi, M., Michalik, H., Moissl, R., Naletto, G., Preusker, F., Sabau, L., Sabolo, W., Scholten, F., Snodgrass, C., Thomas, N., Tubiana, C., Vernazza, P., Vincent, J.-B., Wenzel, K.-P., Andert, T., Pätzold, M., Weiss, B. P., 2011, Images of asteroid (21) Lutetia: A remnant planetesimal from the early solar system, Science, 334, 487-490, http://wWw. sciencemag.org/content/334/6055/487. full.pdf

Soderblom, L., Boice, D., Britt, D., Brown, R., Buratti, B., Kirk, R., Lee, M., Nelson, R., Oberst, J., Sandel, B., Stern, S., Thomas, N., Yelle, R., 2004, Imaging Borrelly, Icarus, 167, 4 - 15, ISSN 0019-1035 
Spjuth, S., Jorda, L., Lamy, P., Keller, H., Li, J.-Y., 2012, Disk-resolved photometry of asteroid (2867) Steins, Icarus, 221, 1101 - 1118, ISSN 0019-1035

Stern, S. A., Parker, J. W., Feldman, P. D., Weaver, H. A., Steffl, A., AHearn, M. F., Feaga, L., Birath, E., Graps, A., Bertaux, J.-L., Slater, D. C., Cunningham, N., Versteeg, M., Scherrer, J. R., 2011, Ultraviolet discoveries at asteroid (21) Lutetia by the Rosetta Alice ultraviolet spectrograph, The Astronomical Journal, 141, 199

Sunshine, J. M., A’Hearn, M. F., Groussin, O., Li, J.-Y., Belton, M. J. S., Delamere, W. A., Kissel, J., Klaasen, K. P., McFadden, L. A., Meech, K. J., Melosh, H. J., Schultz, P. H., Thomas, P. C., Veverka, J., Yeomans, D. K., Busko, I. C., Desnoyer, M., Farnham, T. L., Feaga, L. M., Hampton, D. L., Lindler, D. J., Lisse, C. M., Wellnitz, D. D., 2006, Exposed water ice deposits on the surface of comet 9p/tempel 1, Science, 311, 1453-1455, http://www. sciencemag.org/content/311/5766/1453. full.pdf

Thomas, N., Barbieri, C., Keller, H., Lamy, P., Rickman, H., Rodrigo, R., Sierks, H., Wenzel, K., Cremonese, G., Jorda, L., Küppers, M., Marchi, S., Marzari, F., Massironi, M., Preusker, F., Scholten, F., Stephan, K., Barucci, M., Besse, S., El-Maarry, M., Fornasier, S., Groussin, O., Hviid, S., Koschny, D., Kührt, E., Martellato, E., Moissl, R., Snodgrass, C., Tubiana, C., Vincent, J.-B., 2012, The geomorphology of (21) Lutetia: Results from the OSIRIS imaging system onboard ESA's Rosetta spacecraft, Planetary and Space Science, 66, 96 - 124, ISSN 0032-0633

Vernazza, P., Lamy, P., Groussin, O., Hiroi, T., Jorda, L., King, P., Izawa, M., Marchis, F., Birlan, M., Brunetto, R., 2011, Asteroid (21) Lutetia as a remnant of earth's precursor planetesimals, Icarus, 216, 650 - 659, ISSN 0019-1035

Veverka, J., Helfenstein, P., Lee, P., Thomas, P., McEwen, A., Belton, M., Klaasen, K., Johnson, T., Granahan, J., Fanale, F., Geissler, P., III, J. H., 1996, Ida and dactyl: Spectral reflectance and color variations, Icarus, 120, 66 - 76, ISSN 0019-1035

Veverka, J., Thomas, P., Harch, A., Clark, B., III, J. B., Carcich, B., Joseph, J., Murchie, S., Izenberg, N., Chapman, C., Merline, W., Malin, M., McFadden, L., Robinson, M., 1999, NEAR encounter with asteroid (253) Mathilde: Overview, Icarus, 140, 3 - 16, ISSN 0019-1035

Vincent, J.-B., Besse, S., Marchi, S., Sierks, H., Massironi, M., 2012, Physical properties of craters on asteroid (21) Lutetia, Planetary and Space Science, 66, 79 - 86, ISSN 0032-0633

Weidenschilling, S. J., Davis, D. R., 2001, Planetary Accretion: The First 5 Million Years, in Lunar and Planetary Science Conference, vol. 32 of Lunar and Planetary Inst. Technical Report, p. 1894

Wetherill, G., Stewart, G., 1993, Formation of planetary embryos: Effects of fragmentation, low relative velocity, and independent variation of eccentricity and inclination, Icarus, 106, 190 - 209, ISSN 0019-1035 
Appendix 



\section{A The 2d polynomial fitting to table data of the roughness parameter}

In this appendix, we summarized our try to find an expression for the roughness correction factor $S(\alpha, \bar{\theta})$ used in the Hapke disk-integrated equation. The values of $S(\alpha, \bar{\theta})$ in the phase angle range of $0^{\circ}$ to $180^{\circ}$ are tabulated in Table A.1. The empirical function of

Table A.1: The tabulated values of roughness correction factor $S(\alpha, \bar{\theta})$, taken from Hapke (1993).

\begin{tabular}{cccccccc}
\hline & & \multicolumn{7}{c}{$\bar{\theta}(\mathrm{deg})$} \\
$\alpha(\mathrm{deg})$ & 0 & 10 & 20 & 30 & 40 & 50 & 60 \\
\hline 0 & 1.00 & 1.00 & 1.00 & 1.00 & 1.00 & 1.00 & 1.00 \\
2 & 1.00 & 0.997 & 0.991 & 0.984 & 0.974 & 0.961 & 0.943 \\
5 & 1.00 & 0.994 & 0.981 & 0.965 & 0.944 & 0.918 & 0.881 \\
10 & 1.00 & 0.991 & 0.970 & 0.943 & 0.909 & 0.866 & 0.809 \\
20 & 1.00 & 0.988 & 0.957 & 0.914 & 0.861 & 0.797 & 0.715 \\
30 & 1.00 & 0.986 & 0.947 & 0.892 & 0.825 & 0.744 & 0.644 \\
40 & 1.00 & 0.984 & 0.938 & 0.871 & 0.789 & 0.692 & 0.577 \\
50 & 1.00 & 0.982 & 0.926 & 0.846 & 0.748 & 0.635 & 0.509 \\
60 & 1.00 & 0.979 & 0.911 & 0.814 & 0.698 & 0.570 & 0.438 \\
70 & 1.00 & 0.974 & 0.891 & 0.772 & 0.637 & 0.499 & 0.366 \\
80 & 1.00 & 0.968 & 0.864 & 0.719 & 0.566 & 0.423 & 0.296 \\
90 & 1.00 & 0.959 & 0.827 & 0.654 & 0.487 & 0.346 & 0.231 \\
100 & 1.00 & 0.946 & 0.777 & 0.575 & 0.403 & 0.273 & 0.175 \\
110 & 1.00 & 0.926 & 0.708 & 0.484 & 0.320 & 0.208 & 0.130 \\
120 & 1.00 & 0.894 & 0.617 & 0.386 & 0.243 & 0.153 & 0.094 \\
130 & 1.00 & 0.840 & 0.503 & 0.290 & 0.175 & 0.107 & 0.064 \\
140 & 1.00 & 0.747 & 0.374 & 0.201 & 0.117 & 0.070 & 0.041 \\
150 & 1.00 & 0.590 & 0.244 & 0.123 & 0.069 & 0.040 & 0.023 \\
160 & 1.00 & 0.366 & 0.127 & 0.060 & 0.032 & 0.018 & 0.010 \\
170 & 1.00 & 0.128 & 0.037 & 0.016 & 0.0085 & 0.0047 & 0.0026 \\
180 & 1.00 & 0 & 0 & 0 & 0 & 0 & 0 \\
\hline
\end{tabular}

$S(\alpha, \bar{\theta})$ is only available for $\alpha \leq 60^{\circ}$ (Equation 2.58 in chapter 2). The correction factors are plotted together in Figure A.1 with the values estimated from the empirical expression. It is clear that for $\alpha>60$, the available expression overestimate $S(\alpha, \bar{\theta})$.

In order to remove the deviation for $\left(\alpha>60^{\circ}\right.$, we compute two dimensional polynomial fitting for the roughness correction factors based on its tabulated data by using 


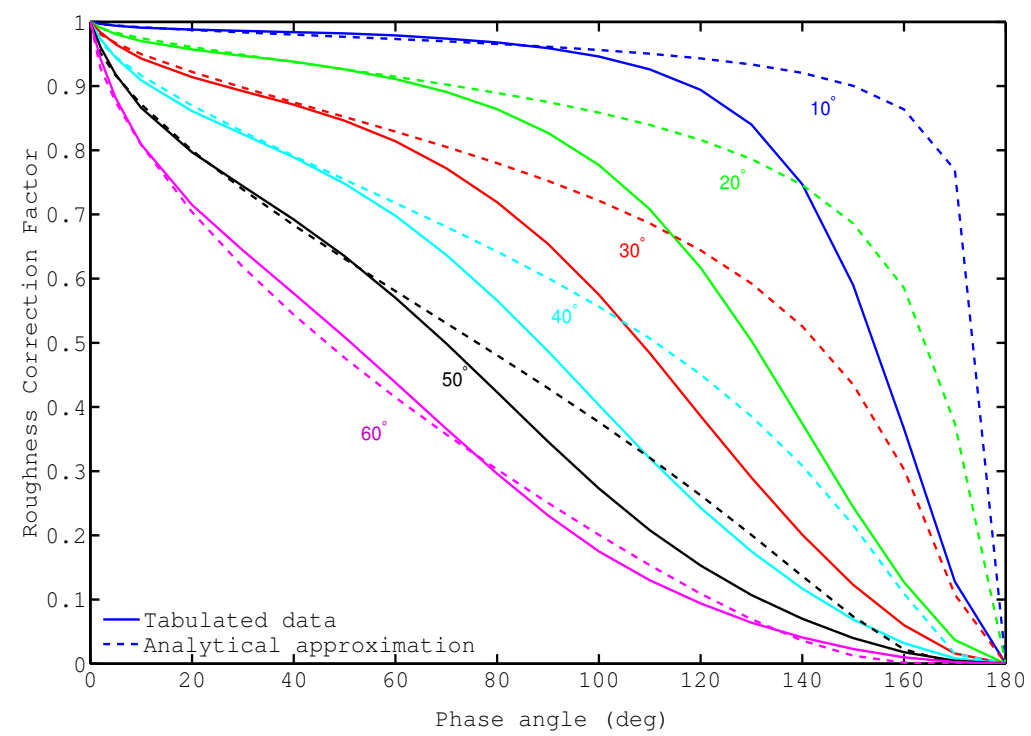

Figure A.1: The plot of the correction roughness factor $S(\alpha, \bar{\theta})$ versus phase angle $(\alpha)$ for different value of $\bar{\theta}$. Courtesy Hapke (1993).

"polyfitn" tools in MATLAB. The result is

$$
\begin{aligned}
S(\alpha, \bar{\theta}) & =k_{1} \bar{\theta}^{6}+k_{2} \bar{\theta}^{5} \alpha+k_{3} \theta^{5} \\
& +k_{4} \bar{\theta}^{4} \alpha^{2}+k_{5} \bar{\theta}^{4} \alpha+k_{6} \theta^{4} \\
& +k_{7} \bar{\theta}^{3} \alpha^{3}+k_{8} \bar{\theta}^{3} \alpha^{2} \\
& +k_{9} \theta^{3} \alpha+k_{10} \theta^{3}+k_{11} \theta^{2} \alpha^{4} \\
& +k_{12} \theta^{2} \alpha^{3}+k_{13} \theta^{2} \alpha^{2} \\
& +k_{14} \theta^{2} \alpha+k_{15} \theta^{2}+k_{16} \theta \alpha^{5} \\
& +k_{17} \theta \alpha^{4}+k_{18} \theta \alpha^{3} \\
& +k_{19} \theta \alpha^{2}+k_{20} \theta \alpha \\
& +k_{20} \theta \alpha+k_{21} \theta+k_{22} \alpha^{6} \\
& +k_{23} \alpha^{5}+k_{24} \alpha^{4}+k_{25} \alpha^{3} \\
& +k_{26} \alpha^{2}+k_{27} \alpha+k_{28}
\end{aligned}
$$

We employed $2 \mathrm{~d}$ polynomial form of roughness correction factor function in fitting process, but the whole Disk-integrated Hapke's eqaution doesn't coverage well and it estimates a negative value for roughness parameter which doesn't have any physical meaning. Therefore, this approach for solving the problem of roughness equation for phase angle larger than $60^{\circ}$ doesn't work properly, and then we decide to use the empirical form of function for whole range of phase angles. 


\section{Scientific contributions}

\section{Refereed contributions:}

- Masoumzadeh, N., Boehnhardt, H., Li, J.-Y., Vincent, J.-B., Photometric analysis of Asteroid (21) Lutetia from Rosetta-OSIRIS images, 2015, Icarus 257, 239-250.

\section{Conference contributions:}

- Masoumzadeh, N., Vincent, J.-B., Sierks, H., Boehnhardt, H., Hofmann, M., The surface topography of asteroid (21) Lutetia, EGU General Assembly, April 2012, Vienna, (poster)

- Masoumzadeh, N., Tubiana, C., Vincent, J.-B., Sierks, H., Photometric analysis of asteroid (21) Lutetia, 44th Lunar and Planetary Science Conference, March 2013, Texas, (poster)

- Masoumzadeh, N., Boehnhardt, H., Li, J.-Y., Vincent, J.-B., Surface reflectance analysis of asteroid (21) Lutetia, ACM Conference, July 2014, Helsinki, (talk) 



\section{Acknowledgements}

First of all, I would like to express my special appreciation and thanks to my supervisor Hermann Böhnhardt, you have been a magnificent mentor for me. I would like to thank you for encouraging my research and for allowing me to grow as a research scientist. I would also like to thank my committee members, professor Ulrich Christensen and professor Stefan Dreizler, whose comments helped me to improve my work.

I would especially like to thank Jian-Yang Li for all the discussion we had mostly via telecommunication and email, for answering to all my technical and physical questions in the field of asteroid photometry. Many thanks to Jean-Baptiste Vincent, who help and answer my questions especially during writing code in MATLAB for developing the tool presented in this thesis.

I want to thank the Max Planck Institute for Solar System Research (MPS) and the International Max Planck Research School for Solar System Science at the University of Göttingen (IMPRS), the former coordinator of the school, the late Dieter Schmitt, and the current one, Sonja Schuh for giving me the opportunity to do my research in such a scientific environment.

Thanks to all my friends and colleagues here in the Institute for all the nice times we shared and for all the valuable discussion we had.

Finally, and most importantly, I would like to thank my parents, my sister and my brother for their support and encouragement. 



\section{Curriculum vitae}

\section{Persönliche Daten}

Name:

Masoumzadeh Jouzdani

Vorname:

Nafiseh

Geburtsort:

Esfahan, Iran

Staatsangehörigkeit: Iranisch

\section{Ausbildung}

2000-2005 BSc in Physik, Isfahan University, Isfahan, Iran

2006-2009 MSc in Physik, Tabriz University, Isfahan, Iran

2011-2015 Promotion an der Universität Göttingen,

Stipendium des Max Planck Institut

für Sonnensystemforschung,

Göttingen, Germany 